\title{
57th Annual ORCA Congress
}

\author{
July 7-10, 2010, Montpellier, France
}

\section{Abstracts}

\section{Contents}

Session 1

172 Epidemiology

Session 2

180 De- and Remineralisation

Session 3

189 Clinical Studies I

Session 4

197 Fluoride

Session 5

205 Microbiology

Session 6

213 Clinical Studies II

Session 7

221 Diagnostics

Session 8

230 Caries Risk

Session 9

238 Erosion

245 Author Index 


\section{Caries Research}

\section{Session 1 Epidemiology}

\author{
1 \\ The Oral Health of Preschool Children with \\ Disabilities in Ireland \\ D. Sagheri ${ }^{a}$ b, * J. Nunn ${ }^{\text {b }}$ \\ darius.sagheri@dental.tcd.ie \\ ${ }^{a}$ Department of Orthodontics, Cologne University Hospital, \\ Germany; ${ }^{b}$ Division of Special Care Dentistry, Dublin Dental \\ School and Hospital, Ireland
}

Background: In Irelands North Eastern (NE) region it is evident that preschool children are today experiencing more dental caries than a decade ago. The plight of preschool children with disabilities is as yet unknown. A prospective, longitudinal study was initiated in order to assess and promote the oral health of preschool children with disabilities. Families with preschool children (0-6 year olds) were recruited for inclusion in the intervention group, provided with a comprehensive oral health promotion programme over a 3 year period and re-assessed in 12 monthly intervals. A matched control group with a similar population was recruited in Irelands South Eastern (SE) region but not provide with oral health promotion. Objectives: The aim of the present report is to inform on the results of the baseline oral health assessment of the two groups. Method: A team of trained and calibrated dentists examined the children's oral health status under same standardized conditions in the two regions. Results: So far, a total of 270 children were examined, 153 (mean age $=3.62$ ) in the NE and 117 (mean age $=3.73)$ in the SE. $92(60.1 \%)$ children in the NE and $56(47.9 \%)$ in the SE were caries free. In each region 5 children had at least one fissure sealant placed on a deciduous or permanent tooth. 86 (56.2\%) of the children in the NE and 34 (29\%) in the SE qualified for referral to the dental hygienist. Conclusion: The present data makes the oral health levels of a high risk group of children transparent who do not usually come within the remit of the dental services in Ireland.

Funding: Health Research Board (Ireland).

\section{* Presenting authors.}

\section{2}

Dental Caries and Oral Hygiene among 11-14-YearOld Children with Mental Retardation in North-West Russia

\author{
M.A. Gorbatova ${ }^{\text {a }}$, M.U. Pastbin ${ }^{\text {b, * }}$, E.I. Proshkina ${ }^{\text {b }}$ \\ mikpastbin@gmail.com \\ ${ }^{a}$ International School of Public Health and ${ }^{b}$ Department \\ of Pediatric Dentistry, Northern State Medical University, \\ Arkhangelsk, Russia
}

In comparison with healthy people, children with mental retardation seem to experience poorer health and less access to professional health care in Russia. To date, dental health status of this vulnerable group has not been examined by using international criteria in northern Russia. The aim of the study was to describe the dental caries experience and oral hygiene level of schoolchildren with mental retardation in North-West Russia. In 2009, 48 children (50\% boys) with mental retardation aged $11-14$ years and attending a Special Secondary School in Arkhangelsk received a dental examination in school. Dental caries was recorded as recommended by the WHO at the D3 level by two calibrated examiners. Oral hygiene level was assessed with the aid of Simplified Oral Hygiene Index (OHI-S) of Green and Vermillion (1967). Caries prevalence is presented with $95 \%$ confidence interval (CI) using the Wilson method. DMFT scores and Significant Caries Index (SiC) are presented as mean \pm standard error. The study was approved by the Ethical Committee of the Northern State Medical University, Arkhangelsk, Russia. On average (SD) the children were 12.6 \pm 1.1 years old. Altogether, $91 \%$ (95\% CI: 80-96) of children had dental caries. Their mean DMFT score was $3.06 \pm 0.34$ and the $\mathrm{SiC}$ index was $5.56 \pm 0.49$. The average numbers of DT, MT and FT were $1.92 \pm 0.32(\mathrm{DT}), 0.10 \pm 0.05(\mathrm{MT})$, and $1.04 \pm 0.2(\mathrm{FT})$. The mean value and SD for OHI-S was $1.6 \pm 0.5$ in the sample under study. No gender differences were observed. Although small sample size cannot provide a very precise estimate of caries prevalence, the findings should raise serious concerns. High levels of decayed and missing teeth may be attributed to the difficulties of this particular group with oral hygiene, and the low proportion of filled teeth may reflect poor organization of dental services. As caries prevalence in 11-14-year old children with mental retardation in Arkhangelsk is higher than in many European countries urgent preventive measures are needed to improve this situation.

\begin{tabular}{ll}
\hline KARGER & @ 2010 S. Karger AG, Basel \\
0008-6568/10/0443-0171\$26.00/0 \\
$\begin{array}{l}\text { Fax +41 61 306 12 34 } \\
\begin{array}{l}\text { E-Mail karger@karger.ch } \\
\text { www.karger.com }\end{array}\end{array}$ & $\begin{array}{l}\text { Accessible online at: } \\
\text { www.karger.com/cre }\end{array}$
\end{tabular}




\section{3}

\section{Dental Caries Related Indicators in Adolescent from Eastern Island, Chile}

R. Cabello *, N. Silva, G. Rodriguez, A. Acuña, I. Urzua

rcabelloi@hotmail.com

Faculty of Denstistry, University of Chile, Chile

In Chile coexist four major ethnics groups (AYMARA, ATACAMEÑO, MAPUCHE and RAPA NUI). RAPA NUI ethnic group are found at Eastern Island. There is a lack of information about dental caries prevalence, $S$. mutans and S. sobrinus counts and oral hygiene evaluation in ethnic communities. The aim of this study is to estimate: caries prevalence, DMFT index, SIC index, oral hygiene index and to evaluate presence of $S$. mutans and S. sobrinus in saliva samples of RAPA NUI school adolescent from eastern island. Study design consists in a cross sectional oral health survey. The study was carried out in 116 randomly selected adolescent with the corresponding informed consent available. One calibrated dentist using natural light and a plane dental mirror performed dental examination. World Health Organization diagnostic criteria were used. Prevalence of caries, DMFT index, and SIC were calculated. Additionally, saliva samples were taken from 23 RAPA NUI individuals for assessment of presence of $S$. mutans and S. sobrinus (PCR) and quantitative counts. $53.3 \%$ of the individuals has dental caries. DMFT Index was 1.43 (95\%CI 1.06-1.81), SIC Index was 3.69 (95\%CI 3.03-4.35). S. mutans and S. sobrinus was found in 56.2 and $13.2 \%$ of the saliva samples respectively. S. mutans mean counts were $3.3 \times 10^{5} \mathrm{CFU} / \mathrm{ml}$ saliva and S. sobrinus mean counts were $7.2 \times 10^{4} \mathrm{CFU} / \mathrm{ml}$ de saliva. No correlation between salivary bacterial levels and caries in the 23 RAPA NUI subjects were observed. In conclusion prevalence of caries, DMFT Index, SIC values suggest to be lower that no RAPA NUI Chilean adolescent.

\section{4 \\ Oral Health Status and Social Class in a Random Sample of 5-Year-Old Brazilian Children \\ S.M. Paiva ${ }^{\mathrm{a}, *}$, K. Bonanato ${ }^{\mathrm{a}}$, M.P. Vale ${ }^{\mathrm{a}}$, M.L. Ramos-Jorge ${ }^{\mathrm{b}}$, F.R. Moura-Leite a, I.A. Pordeus a \\ smpaiva@uol.com.br

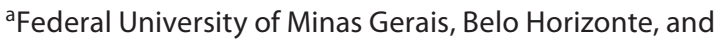 \\ ${ }^{b}$ Federal University of Jequitinhonha and Mucuri Valleys, \\ Diamantina, Brazil}

Oral health status is related to striking differences in different regions of the world. In countries where social discrepancies are pronounced, there is much to be done in order to increase quality of life in all age groups. Among young children, dental caries remains the most frequent oral disease, leading to decreased quality of life for these children and their families. The aim of the present study was to determine the association between oral disease, access to dental care and social class in a random sample in the city of Belo Horizonte, Brazil. A cross-sectional study was conducted on a sample of 551 five-year-old children randomly selected from preschools. Oral health status was assessed using the $\mathrm{dmft}$ index as well as the presence of visible plaque, gingivitis and supra-gingival calculus. Oral examinations were performed by two examiners (Kappa >0.80). The outcome variables were dental caries, filled and missing teeth, dental pulp exposure due to caries, dental root fragment, visible plaque, gingivitis and calculus. Social class was assessed using the City Hall database. Children without caries represented $63.9 \%$ of the sample. Mean overall dmft was 1.56 and the decayed component was the highest in all social classes. Missing teeth, caries with pulp involvement and dental root fragment had higher proportions and the filled component had the lowest proportion in children from the lowest social class. Visible dental plaque was present in $45.4 \%$ of the children. Except for the filled teeth component, all the clinical outcome variables had a significant association to social class $(\mathrm{p}<$ 0.001 ), regardless of child's gender. Oral disease in the primary dentition and access to treatment are affected by social and cultural factors.

Supported by The State of Minas Gerais Research Foundation (FAPEMIG), and by National Council of Technological and Scientific Development (CNPq), Ministry of Science and Technology, Brazil.

\section{5 \\ Trends in Dental Caries Prevalence of Czech Preschool Children over the Last 12 Years \\ E. Lenčová *, Z. Broukal, O. Krejsa \\ lencova@vus.cz \\ Institute of Dental Research - 1st Faculty of Medicine of the Charles University in Prague and General Teaching Hospital in Prague, Czech Republic}

Aim: The aim of the study was to assess trends in caries prevalence of 5-year-olds over the last 15 years based on data from 6 consecutive cross-sectional surveys conducted in the Czech Republic in 1994-2009. Methods: The first 5 surveys, legally supported by the Czech Ministry of Health, were conducted by the Institute of Health Information and Statistics in 1994, 1997, 2000, 2003 and 2006 and included 3,383, 3,578, 3,186, 3,337 and 3,561 subjects, respectively. Dental data were collected from all general dental practitioners in the country and comprised of all 5-yearold patients examined in a dental office within one month. Each patient was included only once. The 6th survey, conducted by the Institute of Dental Research in 2009, included 41 urban and rural sites and recruited 6065 -year-olds based on the approval of Local Ethics Committee and informed consents of the subjects' legal representatives. For each survey, the following parameters were calculated: mean dmft; mean dt and \% of caries-free. For the assessment of the trends, regression lines were plotted for each parameter expressed by values $R$ and $p$ with significance threshold set at $\mathrm{p}<0.05$. Results: Data from 1994, 1997, 2000, 2003, 2006 and 2009: mean dmft: 3.53, 3.61, 3.38, 2.65, 2.69 and 3.04, respectively (ns), mean dt: 2.23, 2.23, 1.85, 1.51, 1.49 and 2.21, respectively (ns), percentage of caries-free: 23.9, 24.7, 29.8, 41.6, 42.2 and 43.99 , respectively $(\mathrm{p}<0.05)$. Conclusions: Over the years $1994-$ 2009 in Czech 5-year-olds, a significant trend of percentage of children with $\mathrm{dmft}=0(\mathrm{R}=0.95, \mathrm{p}<0.05)$ has been observed.

Project NS10599-3/2009 was supported by the IGA of the Ministry of Health of the Czech Republic. 


\section{6}

Interaction between ICDAS II and PUFA Indices in a Child Population in Brasília, Brazil

S.C. Leal ${ }^{\text {a, } *}$, M.J. Figueiredo ${ }^{\text {a }}$, R.G. Amorim ${ }^{\text {a }}$, J. Bittar ${ }^{\text {a }}$, J. Mulder ${ }^{\text {b }}$ J.E. Frencken ${ }^{c}$

J.Frencken@dent.umcn.nl

${ }^{\mathrm{a} D e n t a l}$ School, University of Brasilia, Brazil; ${ }^{\mathrm{b}}$ Cariology,

Endodontology and Paediatric Dentistry, and ' Global Oral

Health, Radboud University Nijmegen, The Netherlands

The aim of this study was to assess the prevalence and severity of dental caries in a child population using the ICDAS II and PUFA indices. The survey was conducted from April-July 2009 in all 6 public schools from Paranoá, a district from Brasília, Brazil. All children registered in grades 1 and 2, who were born between 01.01.2002 and 16.03.2003, were invited to participate. Those whose parents did not sign the informed consent and who presented special needs that did not allow the examination were excluded. A total of 829 children were examined. The prevalence of dental caries in primary dentition, including enamel and dentine carious lesions, was $98.6 \%$. In the primary dentition, the prevalence of code 1 was $65.5 \%$, code 2 was $71.9 \%$, code 3 was $41.1 \%$, code 4 was $13.9 \%$, code 5 was $56.8 \%$ and code 6 was $44.4 \%$. The prevalence of pufa was $23.5 \%$. The prevalence of code $\mathrm{p}$ was $19.3 \%$, code $u$ was $0.1 \%$, code $f$ was $6.6 \%$ and code a was $1.2 \%$. In the permanent dentition, the prevalence of code 1 was $16.8 \%$, code 2 was $10.3 \%$, code 3 was $5.7 \%$, code 4 was $0.2 \%$, code 5 was $3.0 \%$ and code 6 was $0.2 \%$. The restoration components of the ICDAS II index and combinations of the restoration and caries components have not been reported as this would increase the listing of results tremendously. Reporting the results obtained from using ICDAS II alone and in combination with those obtained from using PUFA is difficult and not pragmatic. There is therefore a need to develop a pragmatic reporting system that gives due credit to both indices.

Supported by Fundação de Apoio à Pesquisa do DF - FAP-DF.

7

\section{Evaluation of the Reliability of the Cariogram Software in a Sardinian Population}

G. Campus ${ }^{\text {a, b }, ~ M . G . ~ C a g e t t i ~}{ }^{\text {b }}$, G. Sacco ${ }^{\text {a }}$, S. Sale ${ }^{\text {a }}$ L. Strohmenger ${ }^{\text {b }}$, P. Lingström ${ }^{c, *}$

gcampus@uniss.it

${ }^{a}$ Dental Institute, University of Sassari, bWHO Collaborating Centre of Milan for Epidemiology and Community Dentistry, University of Milan, 'S. Paolo Hospital', Milan, Italy; 'Department of Cariology, Institute of Odontology, Sahlgrenska Academy, University of Gothenburg, Sweden

The aim of this paper was to evaluate the Cariogram reliability by comparing the Cariogram profile at baseline with the actual change in caries disease in the same group during two years. A comparison between the Cariogram and other caries explanatory variables was also done. In 2007, the caries risk profile in a group of Sardinian schoolchildren (957) aged 7-9 years using the
Cariogram software was assessed. Re-examination for caries was done after two years with 862 of the children (drop-out 9.93\%). The children were divided into 5 groups according to the assessed caries risk at baseline. A significant association was observed between the five Cariogram categories recorded at baseline and $\Delta$ caries prevalence $(\mathrm{p}<0.01)$; i.e. where the Cariogram predicted a very low chance to avoid caries (0-20\%) $89.2 \%$ of the subjects in this group showed one or more new lesion, while where Cariogram predicted a very high chance to avoid caries (81-100\%) only one subject $(0.3 \%)$ showed one or more new lesions. Logistic regression (using the presence of one/more new lesions as dependent variable) showed that the Cariogram was the most powerful explanatory variable $(\mathrm{OR}=3.7195 \% \mathrm{CI}=2.82-4.87)$, followed by the presence of salivary lactobacilli $>105(\mathrm{OR}=1.5795 \% \mathrm{CI}=$ 1.23-2.00) and active caries lesions in the permanent dentition recorded at baseline $(\mathrm{OR}=1.1395 \% \mathrm{CI}=1.01-1.28)$. The Cariogram software predicted caries increment more accurately than the other explanatory variables.

\section{8 \\ Is Knowledge of Oral Hygiene and Prevention Related to Caries Experience among Schoolchildren of Montpellier?}

\author{
P. Vintan *, J.C. Chazel, P. Michailesco, P. Tramini \\ vintan.paula@gmail.com \\ Faculty of Odontology, University of Montpellier 1, France
}

In 2008, a school-based oral health education programme was developed in association with the Dental Faculty of Montpellier (France). The aim of this study was to analyze the relationship between oral prevention knowledge and caries experience among schoolchildren aged 5 to 13 years. Taking into account the public/ private ratio, 5 schools of Montpellier were randomly selected, which corresponded to a sampling rate of $1 / 10$ at the school level. All the children (465) attending these 5 schools received a questionnaire adapted to their age group: 5 to 7 years old (G1)/8 to 10 years old (G2)/11 to 13 years old (G3). Different items evaluated the knowledge of oral hygiene practices and the understanding of these instructions. Oral examinations were performed by the same calibrated dentist. The $\mathrm{d} 3+4 \mathrm{ft}$ and D3 $+4 \mathrm{MFT}$ scores were recorded following WHO recommendations. Statistical significance was evaluated by chi-square test for categorical variables and Kruskal-Wallis and Spearman correlation tests for quantitative variables. All authorizations were obtained from the ethic and medical school department. Neither knowledge about oral prevention, nor caries experience significantly differ between girls and boys ( $p=0.34$ and $p=0.20)$. In the youngest group $(\mathrm{G} 1)$, for most of the items (85.7\%), the $\mathrm{d} 3+4 \mathrm{ft}(\mathrm{D} 3+4 \mathrm{MFT})$ scores were lower among children who answered correctly to the questionnaire. In the second group (G2), this percentage was $87.5 \%$. In the oldest group (G3), it decreased to $78.6 \%$. The overall scores of correct answers were significantly correlated to $\mathrm{d} 3+4 \mathrm{ft}$ and D3 + $4 \mathrm{MFT}$ scores in the three groups ( $\mathrm{p}=0.02,0.02$ and 0.04 respectively). We can conclude that knowledge of oral hygiene and prevention is significantly correlated to caries experience in this sample of schoolchildren aged 5 to 13 years. 


\section{9 \\ Three Years Follow-Up Observation of Early Caries Lesion on Occlusal Surface of 1st Molar}

T. Doi *, T. Miyake, M. Uene, K. Jin, K. Kawasaki, M. Kambara

doi@cc.osaka-dent.ac.jp

Osaka Dental University, Osaka, Japan

The purpose of this study was to observe the characteristics of early caries lesions on occlusal surfaces of 1st molar in eighteen elementary school children (6 or 7 years of age) for three years.

Subjects were taking two tablets of Pos-Ca chewing gum (Ezaki Glico Co., LTD, Japan) once a day after lunch for their health promotion. Visual examination was performed by WHO criteria. The 112 sites of early caries lesions on distal and mesial fissures of upper, or distal, mesial and central fissures of lower 1st molars were examined by quantitative light fluorescence (QLF) system (Inspektor-Pro, Inspektor Dental Care, The Netherlands), and $\Delta Q$ value was used as parameter for evaluating early caries lesion. Lesions were classified as progressing (recovering), if initial $\Delta \mathrm{Q}$ increased (decreased) by more than $5 \%$ between baseline and each examination. When the change was less than $5 \%$, a lesion was evaluated as arrested. These examinations were repeated at 6 -month interval throughout a 3 -year study period. The visual examination showed that the $\mathrm{dft}$ index changed from $5.55 \pm 3.45$ to $4.22 \pm 2.62$, and DFT index changed from 0.00 to $0.22 \pm 0.73$ for three years. In the results of reproducibility of $\Delta \mathrm{Q}$ measurement, mean $\Delta \mathrm{Q} \pm \mathrm{SD}$ was $-41.93 \pm 1.76$ from small lesion, and $-213.46 \pm 5.84$ from severe lesion. QLF examination showed that 65 sites of early caries lesions were classified as progressing for first 6-month interval, and $89 \%$ of these lesions were progressing throughout a study period. And recovering lesions were estimated on 40 sites between first 6 months and 55\% of these lesions continued recovering for three years. These findings suggest that the activity of the early caries lesion of three years was affected by a change of the lesion for 6 months.

This study was approved by ODU Dental Ethics Committee (\#060716).

\section{0 \\ Association between Height, Weight, BMI and Dental Caries in 5th Grade Schoolchildren \\ C.H. Splieth ${ }^{\mathrm{a}, *}$, S. Plachta-Danielzik ${ }^{\text {b }}$, M.J. Müller ${ }^{\mathrm{b}}$ \\ splieth@uni-greifswald.de \\ aDepartment of Preventive and Pediatric Dentistry, University \\ of Greifswald, and ${ }^{\mathrm{b}}$ Institute for Human Nutrition and Food \\ Science, Kiel, Germany}

General and dental health are interdependent. Apart from the cariogenic aspect of our diet, proper nutrition is essential for growth and development and first studies on relationship between height and dental caries have been published [Freire et al.; Caries Res. 2008;42:134-409]. Thus, the aim of this analysis was to evaluate a possible association between height, weight, BMI and dental caries in children. The sample comprised 852 schoolchildren in 5 th grade $(9-13$ years, mean $10.8 \pm 0.5)$ in Greifswald and Pomerania. In conjunct with the compulsory, dental commu- nity examination, standardized measures of height, weight and waist circumference were taken, the BMI was calculated and correlations with the dental caries (DMFT/S, dmft/s) were analysed (Spearman-Rho coefficient). The distribution of the DMFT values was highly polarized with most of the children exhibiting no carious defects, fillings or missing teeth in the permanent dentition (mean $0.6 \pm 1.2 \mathrm{DMFT}$ ). Caries in deciduous teeth was more prevalent (mean $1.4 \pm 1.9 \mathrm{dmft}$ ). The BMI and the nutritional status indicated more overweight than underweight. The BMI and caries parameters did not have any statistically significant association. After adjusting for age, correlations between waist circumference and caries were not statistically significant. Only weight and especially height showed persistent correlations with caries in the deciduous $(-0.12<$ Spearman-Rho coefficient $<-0.26, \mathrm{p}<0.05)$, but not in the permanent dentition $(0.12<\mathrm{p}<$ $0.34)$. This was confirmed in a regression analysis including age and gender (coefficient $\mathrm{b}-0.041, \mathrm{p}<0.01$ ). In conclusion, growth and development in children seem to be a strong confounding factor for the association between caries and height/weight for time period of mixed dentition. Still, studies in other age groups and social structure would be helpful to gain more insight in the associations between dental and general health status.

Supported by the Federal Ministry for Health in Gemany (FKZ: 01EL0610).

\section{1 \\ Intra-Familial Comparison of Caries Prevalence and Associated Factors in a Saudi Population}

\section{A. Mannaa *, A. Carlén, P. Lingström}

alaa.mannaa@odontologi.gu.se

Institute of Odontology, University of Gothenburg, Sweden

The aim of this cross-sectional study were to describe the prevalence of dental caries and associated factors in Saudi mothers and their children, and to examine possible correlations between the mothers and their children. Totally, 258 subjects ( 86 mothers and two of their children, $4-6$ years and $12-16$ years old) were included in the study. Semi-closed questionnaires were used to obtain data about their oral health, socio-economic status, oral hygiene habits, and dietary habits. Dental caries was recorded clinically and radiographically according to the WHO standards. The amounts of dental plaque and gingivitis were scored. Chairside tests were used to obtain the numbers of mutans streptococ$\mathrm{ci}$ and lactobacilli and buffer capacity in saliva. The caries experience $(\mathrm{DMFT} / \mathrm{dmft}$, mean $\pm \mathrm{SD})$ was $12.4 \pm 5.3,9.0 \pm 5.0$ and $5.8 \pm 4.1$ for the mothers, $4-6$ years and $12-16$ years old children respectively. $4 \%$ of mothers, $9 \%$ of younger children, and $12 \%$ of older children were caries free. Caries prevalence of children followed that of their mothers $\left(\mathrm{R}^{2}{ }_{4-6}=0.117 ; \mathrm{R}^{2}{ }_{12-16}=0.182, \mathrm{p}<0.01\right)$. The prevalence decreased in relation to mother's education and increased in relation to number of children per family (n.s.). A positive correlation for plaque scores was found between mothers and older children $\left(\mathrm{R}^{2}=0.186, \mathrm{p}<0.01\right)$. Gingivitis scores, brushing habits and snack consumption were correlated between mothers and their children (gingivitis: $\mathrm{R}_{4-6}^{2}=0.079 ; \mathrm{R}_{12-16}^{2}=0.219$, $\mathrm{p}<0.01$; brushing: $\mathrm{p}<0.05$; snacking: $\mathrm{R}_{4-6}^{2}=0.137$; $\mathrm{R}_{12-16}^{2}=$ $0.118, \mathrm{p}<0.01)$. Buffer capacity and mutans streptococci counts were correlated between mothers and older children $(B C$ : $p<0.01$; 
MS: $\mathrm{p}<0.01)$. To conclude, the caries experience in Saudi children is related to caries and associated factors in their mothers.

Supported by The Saudi Ministry of Higher Education

\section{2}

\section{Frequency and Risk Factors for Dental Caries among} Pregnant Women in France

\author{
J.N. Vergnes ${ }^{\text {a, }}{ }^{*}$, M. Kaminski ${ }^{\text {b }}$, N. Lelong ${ }^{\text {b }}$, A.M. Musset ${ }^{c}$, \\ M. Sixou ${ }^{\mathrm{C}}, \mathrm{C}^{\mathrm{C}}$ Nabet ${ }^{\mathrm{b}}$ \\ jn.vergnes@free.fr

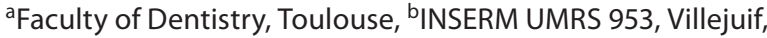 \\ 'Faculty of Dentistry, Strasbourg, France
}

Objectives: Dental care for pregnant women is usually delicate, with contraindications and necessity for multiple precautionary measures. Little is known on the prevalence of dental caries among pregnant women. The objectives were to assess the frequency and number of dental carious lesions among a large sample of pregnant women in France, and to study associated risk factors. Methods: A dental examination was performed within 2 to 4 days post-partum in a sample of 1,094 at-term women of six maternity units. The number of dental carious lesion was recorded. Socio-demographic and behavioural characteristics were obtained through a standardised interview with the women: age, nationality, marital status, educational level, employment during pregnancy, smoking status during pregnancy and adequacy of prenatal care. A negative binomial hurdle model was used to analyse the risk factors associated with both the frequency and the number of dental carious lesions. Results: $51.6 \%$ of the women presented at least one dental carious lesion. This frequency was statistically associated with lower age $(\mathrm{aOR}=1.65,95 \% \mathrm{CI}$ [2.15-2.51]) and lower educational levels $(\mathrm{aOR}=1.61,95 \% \mathrm{CI}[1.13-2.29])$. The number of dental carious lesions was associated with lower age, lower educational level and non-French nationality $(\mathrm{p}<0.01)$. Conclusions: We showed in this sample of pregnant women that frequency and number of dental carious lesions were very high. As dental caries is a preventable disease, future oral health promotion programmes must continue to inform women and providers about the importance of dental care before and during pregnancy.

Supported by National Programme for Hospital Clinical Research (national PHRC2004, AOM04047) INSERM (National Institute of Health and Medical Research).

\section{3 \\ Root Caries Experience in a Nursing Home Population \\ M.J. Narayan a , M.S. Hopcraft ${ }^{\mathrm{a},}$, E. Milford ${ }^{\mathrm{b}}$, M.V. Morgan ${ }^{\mathrm{a}}$ \\ m.hopcraft@unimelb.edu.au \\ a Melbourne Dental School, The University of Melbourne, and \\ ${ }^{b}$ Dental Health Services Victoria, Melbourne, Australia}

Aim: The aim of this study was to measure root caries experience in a group of elderly Australians living in residential aged care facilities (RACFs), and investigate associations with salivary parameters. Methods: Clinical dental examinations were conducted on a group of nursing home residents from 20 RACFs in metropolitan Melbourne. ICDAS-II criteria were used to assess coronal and root caries, with root caries assessed on buccal and lingual surfaces of teeth. Saliva parameters were measured using the GC Saliva Check Kit, and xerostomia measured using the Xerostomia Inventory. Results: A total of 221 dentate residents had dental examinations, with a mean 14.2 teeth present. The mean decayed root surfaces (DRS) were 4.54, 4.44 and 5.81 for residents aged 60-79 years, $80-89$ years and $90+$ years of age respectively, and only $23.5 \%$ of residents had no root caries experience. Residents with a salivary flow rate $>1.0 \mathrm{ml} / \mathrm{min}$ had a mean $3.84 \mathrm{DRS}$, compared with 6.36 for those with $<0.7 \mathrm{ml} / \mathrm{min}$ ( $\mathrm{p}=0.061$ ). Poisson regression found that root caries experience was strongly associated with resting and stimulated $\mathrm{pH}$, and saliva flow rate. However, there was no association with self-reported xerostomic status. Conclusion: Dentate residents of nursing homes in Melbourne had a high mean root caries experience, with nearly 5 surfaces affected.

Supported by Alzheimer's Australia Hazel Hawke Research Grant and Dental Health Services Victoria Research Grant.

\section{4 \\ Dental Caries Experience in a Nursing Home Population}

\section{M.J. Narayan *, M. S. Hopcraft, E. Milford, M.V. Morgan}

m.narayan1@pgrad.unimelb.edu.au

Melbourne Dental School, The University of Melbourne, Australia

Aim: The aim of this study was to measure dental caries experience in Australians living in residential aged care facilities (RACF), and investigate associations with epidemiological factors. Methods: Clinical dental examinations were conducted on residents from 20 RACF in Melbourne. Three dental examiners, using a mouth mirror, probe and headlamp assessed coronal dental caries using ICDAS-II criteria. Sociodemographic data was collected via questionnaire. Results: Of 427 residents assessed, 221, (66 male and 155 female) were dentate. The majority, 107 residents, were $80-89$ years old, with 60 residents aged $60-79$ and 48 residents aged $90+$ years. $80 \%$ of residents were pension cardholders, $60 \%$ were privately insured. $55 \%$ had a diagnosed dementia, $30 \%$ a history of stroke and $20 \%$ had diabetes. Dentate residents had a mean 14.2 teeth present with a mean DMFT of 21.69. Mean DMFT increased with age, from 20.63 in the 60-79 age group, to 22.18 in the $80-89$ group and 22.83 in the $90+$ group. Untreated coronal caries was common, with mean DT of 2.28, 2.83 and 3.19 for residents aged $60-79$ years, $80-89$ years and $90+$ years respectively. The younger group had higher mean FT (5.8) than the older groups (4.87 for the 80-89 and 5.14 for the $90+$ residents). Female residents had a significantly higher DMFT of 22.56 than males with a DMFT of $19.70(\mathrm{p}<0.05)$, and more missing teeth. Pension cardholders had significantly more missing teeth, fewer teeth present and fewer filled teeth than non-pension cardholders. Privately insured residents had significantly more filled teeth. There were no associations between DMFT and comorbid dementia, diabetes or stroke. Conclusion: Dentate residents of aged care facilities in Melbourne had a high level of dental caries experience, with patterns modified by gender, age and 
other social factors. High levels of untreated coronal caries suggest poor access to dental care.

Supported by Alzheimer's Australia Hazel Hawke Research Grant and Dental Health Services Victoria Research Grant.

\section{5 \\ Caries Experience among Schoolchildren in South-Eastern Nigeria \\ L.O. Okoye * \\ drogeokoye@yahoo.co.uk \\ University of Nigeria Enugu Campus, Enugu, Nigeria}

Introduction: Dental caries amongst children poses a public health problem in the developed countries. A number of studies have been carried out to determine its prevalence. However, there is paucity of data generated from South-Eastern Nigeria. This study hopes to contribute to data in the sub-Saharan region. Objectives: This study was carried out to assess dental caries in 12 15 year old children in Enugu, a major city in South-Eastern Nigeria. Methods: A multistage sampling technique was used to select 400 students. Questionnaires were administered and participants were examined for dental caries using the Decayed, Missing and Filled Teeth index criteria according to WHO guidelines. The resultant data was analyzed using SPSS version 10 . Results: There were 169 males (42.2\%) and 231 females (57.8\%); $15.5 \%$ has had dental caries. Mean DMFT score was $0.16 \pm 1.24$. Decayed teeth accounted for the largest component (85.5\%), while only $3 \%$ of the caries were restored. Prevalence was higher in females (18.6\%) than in males (11.2\%). Percentage DMFT was found to increase with age. No statistical significant association was found between caries and mother's educational level. Most children $(84.5 \%)$ had never visited the dentist. Conclusion: There is low caries experience in Enugu. Untreated caries indicate poor dental awareness and high restorative treatment needs in SouthEastern Nigeria. There is therefore need for public educational and restorative dental programmes.

\section{6}

\section{Correlation between Potential Risk Factors and Caries Experience among Children and Adults}

P.Y. Collart Dutilleul ${ }^{a},{ }^{*}$, R. Chave a , J. Valvarcel a , P. Tramini a, J. Véronneau ${ }^{\mathrm{b}}$

pycd@ hotmail.fr

aUFR d'Odontologie, Université de Montpellier, France;

bMcGill University, Montreal, Canada

Risk assessment is an essential component in the decisionmaking process for the correct prevention and management of dental caries. There is a lack of adult studies on caries risk assessment (CRA). Study Aim: This cross-sectional piloted study is using an international multisite CRA cohort designed project to explore the correlation between potential risk factors and dental caries experience among a sample of children and adults aged 4 to 82 years. Method: One trained dentist examined all voluntary participants having minimally 20 teeth, in a dental conventional academic setting. Caries experience - dmfs - was the sum of dental surfaces with obvious dentinal lesion, filling or missing for caries. An empirical grid of ten potential risk factors all selected based on literature, has been created with dichotomised (yes/no) questions on socioeconomic status, habits and clinical initial and dentinal lesion observations. All risk factors were weighed in term of risk using specific score: clinical factors had higher attributed weighted score than non-clinical risk. Four risk subcategories were created based on subject total score. Main Results: A sample of 77 participants aged 32.4 in average had a dental exam and grid-questionnaire completed. The mean dmfs was 32.05 (30.67) for the whole sample. Categorical scores generated were 0-4, 5-9, 10-14 or 15 and more (range: $0-21$ ). Spearman tests were significant to describe the correlation between $\mathrm{dmfs}$ and each specific risk-score category. The significant correlation was maintained between the specific $\mathrm{dmfs}$ and its corresponding risk-category. Conclusions: Grouping and grading caries-risk factors generated significant associations with the corresponding experience of caries.

Supported by the Dental Faculty of Montpellier, France, and MacGill University, Montréal, Canada.

\section{7 \\ Oral Health Status of Indigenous People Living in Remote Communities in Central Australia \\ G.G. Adams ${ }^{\text {a, }}$, A. Lee ${ }^{\text {b }}$, M.J. McCullough ${ }^{\text {b }}$, G.L. Borromeo ${ }^{\text {b }}$ \\ g.adams@unimelb.edu.au \\ a Oral Health CRC, Melbourne Dental School, The University of Melbourne, and belbourne Dental School, The University of \\ Melbourne, Australia}

Aim: Australians experienced more than three times the rate of untreated coronal decay and more missing teeth than the nonIndigenous population. However, this survey was conducted predominantly in metropolitan and regional centres and not in remote communities where $89 \%$ of Indigenous people in Northern Territory live. The aim of this study was to assess the current oral health status in Indigenous Australians living in remote communities in Central Australia. Methods: 126 adult participants (40\% male, age range 18-74 years) from five remote Central Australian Indigenous communities were recruited. Ethics approval was obtained from the Central Australian Human Research Ethics Committee and informed consent obtained from participants prior to the oral examination. Comprehensive oral examinations were conducted from May to July 2009 in a mobile dental van by a single experienced examiner (AL). Results: Mean DMFT was 7.0 $\pm 6.3,67 \%$ of participants were missing at least one tooth, $59 \%$ of participants had untreated coronal decay and $49 \%$ had fillings. Mean decayed and filled surfaces were $5.5 \pm 9.4$ and $2.3 \pm 3.5$, respectively. The number of untreated coronal decayed surfaces was approximately double that observed for the Indigenous population and more than 6 times that observed for the non-Indigenous population in the national survey, whilst the number of filled surfaces was at least 6 times greater in the national survey. Conclusion: This study showed an overall higher prevalence of untreated decay in the remote Indigenous communities than that observed in Indigenous and non-Indigenous Australians living in metropolitan and regional centres.

Supported by Northern Territory Oral Health Service, Melbourne Dental School and Oral Health CRC. 


\section{8 \\ Trends towards Improving Dental Health Outcomes in Glasgow and Scotland: Caries Epidemiology 1993/94-2007/08}

Y.I. Blair a , L.M.D. Macpherson ${ }^{\text {b, }}{ }^{*}$, A.D. McMahon ${ }^{b}$, D.R. McCall ${ }^{\text {a }}$

I.macpherson@dental.gla.ac.uk

${ }^{a}$ NHS Greater Glasgow \& Clyde, ${ }^{\text {b University of Glasgow Dental }}$

School, Glasgow, UK

Aim: To assess longitudinal trends in caries outcomes in Scotland and Glasgow against a background of ecological oral health promotion (OHP) programmes. Methods: Trained and calibrated (using BASCD criteria) dentists have carried out biennial caries epidemiological surveys among Scottish schoolchildren in their first elementary P1 school year under the Scottish Health Boards Dental Epidemiological Programme (SHBDEP) and successor National Dental Inspection Programmes (NDIP), since 1987. Children's home postcode sector data has been collected from 1993/94. Historic datasets were retrieved from the Data Holders and a master caries database at tooth-surface level was compiled. Secondary data analysis assessed trends in mean $\mathrm{d} 3 \mathrm{mft}$ and in prevalence of $\mathrm{d} 3 \mathrm{mft}=0$ over eight cross-sectional surveys (1993/94-2007/08). Additional to analysis at the whole of Scotland level, geographic and SES subgroup analyses were performed. Results: Data from 68,398 children [mean age 5.4 (95\%CI 5.4-5.5) years] were included. Overall, in Scotland and Glasgow, respectively, the SES adjusted mean $\mathrm{d} 3 \mathrm{mft}$ decreased over the period, from 3.08 (95\%CI $2.99-3.18)$ to 1.86 (95\%CI $1.81-1.91)$ and 3.85 (95\%CI 3.57-4.12) to 2.17 (95\%CI $2.07-2.27)$ while the \% adjusted $\mathrm{d} 3 \mathrm{mft}=0$ increased concurrently from $41 \%$ (95\% CI $40-42)$ to $58 \%(95 \%$ CI $58-59)$ and from $32 \%$ (95\%CI $28-35)$ to $54 \%(95 \% \mathrm{CI}$ $53-56)$. In all cross-sectional surveys marked SES gradients were apparent (by DepCat, 2001), the respective relationship was direct for mean $\mathrm{d} 3 \mathrm{mft}$ score and indirect for $\% \mathrm{~d} 3 \mathrm{mft}=0$. From baseline, by $2007 / 08$, the absolute value of improvements in $\% \mathrm{~d} 3 \mathrm{mft}=0$ in Scotland's DepCat 1 and DepCat 7 children in the age-group achieved 12.1 and 21.4 percentage points, respectively, whilst in Glasgow the corresponding values were 26.6 and $24.1 \%$. Conclusion: These findings suggest benefits are accruing to P1 children from OHP in Scotland. There are implications for future OHP programme design, especially in relation to the balance between universal and targeted interventions.

Supported by NHS Scotland \& the Scottish Government.
19

Selected Tests of Dental Health Inequalities in 5-Year-Olds' Dental Health in Glasgow and Scotland: 1999/00-2007/08

Y.I. Blair ${ }^{\mathrm{a}, *}$, L.M.D. Macpherson ${ }^{\mathrm{b}}$, A.D. McMahon ${ }^{\mathrm{b}}$, D.R. McCall ${ }^{\mathrm{a}}$

y.blair@dental.gla.ac.uk

${ }^{a}$ NHS Greater Glasgow and Clyde, b University of Glasgow Dental

School, Glasgow, UK

Aim: To assess dental health inequalities in Glasgow and Scotland against a background of improving population dental health among 5-year-olds and a Scottish Government (S.G.) target to improve population health and reduce SES inequalities. Methods: Selected tests of dental health inequality, namely, simple absolute range, simple relative range, SIC10, the Gini-coefficient, the Transformed Concentration Index (TCI), the Slope Index of Inequality (SII), Relative Index of Inequality (RII) and the Population Attributable Risk (PAR) were applied to the same 43,715 children's caries data from SHBDEP \& NDIP surveys, 1999/002007/08. Results: In Glasgow, the respective overall reductions in d3mft inequalities were as follows, 1999/00-2007/08: simple absolute inequality (in mean d3mft, 3.2 to 2.5), SIC10 (12.30 to 9.85), TCI (10.7 to 10.0), SII (5.17 to 2.64), RII (1.44 to 1.24 ) and PAR\% (72.59 to 49.10). Meanwhile, increases in overall d3mft inequalities were observed in simple relative inequality (in mean $\mathrm{d} 3 \mathrm{mft}$, 2.6 to 5.2) and the Gini-coefficient. Simultaneously, in Scotland, respective tests indicating decreased $\mathrm{d} 3 \mathrm{mft}$ inequality were: simple absolute inequality (in mean $\mathrm{d} 3 \mathrm{mft}, 3.7$ to 2.5 ), SIC10 (10.43 to 9.27), SII (2.93 to 2.24) and PAR\% (32.04 to 22.79), whilst inequality increased in simple relative inequality (in mean $\mathrm{d} 3 \mathrm{mft}, 3.8$ to 4.6), TCI (8.9 to 9.8$)$, RII (1.15 to 1.17) and the Gini-coefficient. Discussion: Concurrent multiple inequalities tests are advocated for general health by the S.G. These results demonstrate that the inequalities outcomes with population dental health improvements vary depending on the inequality test adopted. Over and above the simple tests, the SIC10, TCI, SII, RII and PAR each produced additional inequalities information from $\mathrm{d} 3 \mathrm{mft}$ data. However, Gini-coefficient results were confusing and counterintuitive. Conclusion: A more complete future understanding of caries inequalities trends will be gained from application of multiple selected inequalities tests.

Supported by NHS Scotland \& the Scottish Government. 


\section{0}

\section{Caries Prevalence and Tooth Surface Distribution in a Group of Chilean Preschool Children}

G. Rodríguez *, R. Cabello, I. Urzúa, G. Zillmann

gonzalo.a.rodriguez@gmail.com

Faculty of Dentistry, University of Chile, Santiago, Chile

The aim of this study was to contribute to the descriptive information on oral health status of preschool children from the Metropolitan Region of Chile. Study design consists in a cross sectional oral health survey. The study was carried out in 382 randomly selected 2 to 6 years old preschool children with the corresponding parental informed consent available. One calibrated dentist using artificial light and a plane dental mirror performed dental examination. World Health Organization (WHO) diagnostic criteria were used to assess tooth surfaces. Prevalence of caries at individuals, tooth and surface level were calculated. Also $\mathrm{dmft}, \mathrm{dmfs}$ and Sic were calculated. Prevalence at individual level was 48.95\% (95\% CI 43.89-53.99). The most commonly affected teeth were primary molars ( $77 \%$ of the overall sample), especially in the mandible. The most commonly affected surfaces were the occlusal surfaces of primary lower molars. The $\mathrm{dmft}$ calculated was 2.47 (95\% CI 2.13-2.81), and the dmfs was 3.98 (95\% CI 3.34$4,61)$. The significant caries index was 6.61 (95\% CI 6.14-7.07). In conclusion, caries experience in deciduous dentition showed a symmetrical distribution at the surface and tooth level.

\section{1 \\ Paper and Electronic Case Report Forms for Epidemiological Studies in Dentistry}

\author{
J.C. Carvalho ${ }^{\mathrm{a}, *}$, P. Bottenberg ${ }^{\text {b }}$, D. Declerck ${ }^{c}$, J. Vanobbergen ${ }^{d}$, \\ J.P. Van Nieuwenhuysen ${ }^{a}$, M. Nyssen ${ }^{b}$ \\ joana.carvalho@uclouvain.be \\ ${ }^{\mathrm{a} C}$ Catholic University of Louvain, Brussels, ${ }^{\mathrm{b}}$ Free University of \\ Brussels, 'Catholic University of Leuven, dUniversity of Gent, \\ Belgium
}

The Belgian National Institute of Health Insurance is implementing an oral health data registration system. In the framework of such a system, a Case Report Form was developed both in paper and in electronic format. The aim of this study was to validate data capture for both formats of the Case Report Form. Six series of full mouth recordings simulating the clinical examination of 6 patients were set up in a Powerpoint presentation. The validation was undertaken by a sample of 52 private practitioners (2276 -year-old) from 39 municipalities. Firstly, the dentists were randomly assigned to start data capture either with the Paper Case Report Form (PCRF) or the Electronic Case Report Form (ECRF). Secondly, the data capture was crossed-over. Thirdly, benchmarks of PCRF were transferred to ECRF. The forms were verified for correctness, completeness and time in minutes spent to capture and process the data. The dentists answered an electronic questionnaire about the PCRF and ECRF. The overall percentages of error were 25.3\% (PCRF), 26.6\% (ECRF) and $18.9 \%$ (benchmark of PCRF transferred). Very good completeness (5\% of incorrect completeness) was identified in $93 \%$ of the forms. The
Signed Rank Test showed a significant difference in the time spent to capture and process the data between ECRF and PCRF (3.2 min versus $4.6 \mathrm{~min}, \mathrm{p}<0.001)$. Two thirds of the dentists preferred the PCRF to the ECRF with no significant difference in relation to age $(\mathrm{p}=0.21)$. In conclusion, the Case Report Form was validated for all types of data capture that were tested. As information technology has turned into an evermore necessary working tool in general practice, there should be an important potential for uptake of further improvements in electronic data capture in the future.

\section{2 \\ Relationship between Toenails Fluoride Concentrations and Caries Prevalence Using the ICDAS-II Criteria

\author{
S. Charone ${ }^{\mathrm{a}, *}$, R.M. Gonçalves ${ }^{\mathrm{b}}$, C. Cloivos ${ }^{\mathrm{b}}$, L. Grizzo ${ }^{\mathrm{a}}$, \\ M.A.R. Buzalaf ${ }^{\text {, }}$ S. Groisman ${ }^{\text {b }}$ \\ sendacharone@yahoo.com.br \\ aBauru School of Dentistry, University of São Paulo, Bauru,

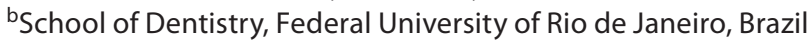

This study examined the relationship between fluoride concentrations in toenails and prevalence of caries using the International Caries Detection and Assessment System (ICDAS-II) criteria. Fifty-four children (4-13 years of age) living in Rio de Janeiro, Brazil, had their teeth surfaces examined by 2 calibrated examiners (Kappa inter-examiner $=0.72-0.80$ ) using ICDAS-II criteria. Following examination, toenails were clipped and analyzed for fluoride with the electrode (Orion 9409), after hexamethyldisiloxane diffusion. Toenails fluoride concentrations in children presenting ICDAS-II $\leq 10$ or $>10$ were compared by unpaired $t$ test with Welch correction. Dichotomized data were analyzed by Fisher's exact test. The significance level was set at $5 \%$. Mean ICDAS-II ( \pm SD, 95\% CI) was 28.3 ( $\pm 40.2,17.3-39.3)$. Mean \pm SD toenails fluoride concentrations in children $(\mathrm{n}=23)$ presenting ICDAS-II $\leq 10(1.85 \pm 1.32 \mu \mathrm{g} / \mathrm{g})$ were higher than values found those $(\mathrm{n}=31)$ having ICDAS-II $>10(1.58 \pm 0.78 \mu \mathrm{g} / \mathrm{g})$, but the difference was not significant. The corresponding $95 \% \mathrm{CI}$ were 1.28-2.42 and 1.29-1.87 $\mu \mathrm{g} / \mathrm{g}$, respectively. Among children having ICDAS-II $\leq 10,5$ had toenails fluoride concentrations higher than $2.0 \mu \mathrm{g} / \mathrm{g}$, while 18 had concentrations equal to 2.0 $\mu \mathrm{g} / \mathrm{g}$ or lower. For those presenting ICDAS-II $>10$, the corresponding figures were 7 and 24, respectively. The sensitivity and specificity of toenails fluoride concentrations in identifying children with ICDAS-II $\leq 10$ was 0.22 and 0.77 , respectively. The positive and negative predictive values were 0.42 and 0.57 , respectively. In conclusion, children with low caries prevalence tend to have higher toenails fluoride concentrations, but the validity of this biomarker as a diagnostic tool for caries prevalence is low, probably due to the fact that the mechanism of action of fluoride on caries control is essentially topical.

Supported by FAPESP. 


\section{Caries Research}

\section{Session 2 \\ De- and Remineralisation}

\section{3 \\ In vitro Remineralization of White-Spot Lesions by Fluoride Rinses with and without Functionalized Tricalcium Phosphate \\ R.L. Karlinsey ${ }^{\mathrm{a}, *}$, A.C. Mackey ${ }^{\mathrm{a}}$, G.K. Stookey ${ }^{\mathrm{b}}$ \\ rkarlins@gmail.com \\ andiana Nanotech, ${ }^{\mathrm{b}}$ Therametric Technologies, Indianapolis, Ind., USA}

The aim of this work was to determine the feasibility of combining a functionalized tricalcium phosphate (fTCP) system with a $0.05 \% \mathrm{NaF}$ commercial aqueous rinse formulation (ACTas a means of enhancing remineralization of early caries lesions in vitro. The studies included $\mathrm{pH}$ cycling and enamel fluoride uptake and involved the following groups: A: distilled (DI) water; $\mathrm{B}$ : ACT; C: ACT + 30 ppm fTCP; and, D: ACT + 60 ppm fTCP. Whitespot lesions were initially formed in bovine enamel specimens $(\mathrm{n}=10)$ immersed in a carbopol-lactic acid solution for $36 \mathrm{~h}\left(37^{\circ} \mathrm{C}\right)$ and then subjected to a $\mathrm{pH}$ cycling study with each day consisting of four two-minute treatments, one four-hour acid challenge (carbopol-lactic acid, $\mathrm{pH}=5.0$ ), and artificial saliva $(\mathrm{pH}=7.0)$ in between these events. After six days of cycling, mean \pm SEM changes in surface microhardness were A: $-1.2 \pm 0.9^{\mathrm{a}} ; \mathrm{B}$ $21.4 \pm 1.3^{\mathrm{b}}$; C: $32.8 \pm 2.8^{\mathrm{c}}$; and D: $27.8 \pm 3.3^{\mathrm{b}, \mathrm{c}}$, where $\mathrm{a}<\mathrm{b}<\mathrm{c}$ (ANOVA, SNK, p < 0.05). In a separate study, bovine enamel specimens $(\mathrm{n}=12)$ were immersed in carbopol-lactic acid solution $(\mathrm{pH}$ $=5.0$ ) for $24 \mathrm{~h}\left(37^{\circ} \mathrm{C}\right)$ and subjected to a single 30 -minute treatment. The mean \pm SEM enamel fluoride uptake $\left(\mu \mathrm{g} \mathrm{F} / \mathrm{cm}^{3}\right)$ were A: $44.9 \pm 4.4^{\mathrm{a}}$; B: $1056.5 \pm 58.0^{\mathrm{b}}$; C: $1324.8 \pm 81.6^{\mathrm{c}}$; and, D: 1314.9 $\pm 46.7^{\mathrm{c}}$, where $\mathrm{a}<\mathrm{b}<\mathrm{c}($ ANOVA, SNK, $\mathrm{p}<0.05)$. Based on the significance of these results, along with supporting fluoride stability data and optical microscopy and Knoops microhardness assessments of $\mathrm{pH}$ - cycled enamel cross-sections, we conclude the addition of fTCP to a $0.05 \% \mathrm{NaF}$ aqueous rinse establishes in vitro feasibility support for continued development and clinical evaluation of an fTCP-NaF rinse system for enhanced remineralization.

Supported by grant number R43DE018576 from the National Institute of Dental \& Craniofacial Research.

\author{
24 \\ Studying Enamel De-/Remineralisation under \\ Plaque Fluid-Like Conditions - A QLF Study \\ F. Lippert ${ }^{\mathrm{a}, *}{ }^{*}$, R.J.M. Lynch ${ }^{\mathrm{b}}{ }^{\mathrm{b}}$ A. Butler ${ }^{\mathrm{b}}$ \\ flippert@iupui.edu \\ andiana University School of Dentistry, Indianapolis, USA; \\ ${ }^{\mathrm{b}}$ GlaxoSmithKline, Weybridge, UK
}

The aim was to investigate de- and remineralisation in enamel lesions under plaque fluid-like conditions using QLF. Lesions were formed in bovine enamel using the acid-gel method $(0.1 \%$ lactic acid; $\mathrm{pH} 4.6 ; 12$ days) and randomised into 15 treatment groups $(n=9)$ based on $\Delta$ Fbase $(-55.6 \pm 6.2 \%)$. Lesions were then exposed to solutions containing $30 \mathrm{~mm}$ lactic acid, $5.5 \mathrm{~mm} \mathrm{Ca}, 9.4$ $\mathrm{mM} \mathrm{Pi}, 63 \mathrm{mM} \mathrm{KCl}$, varying in $\mathrm{pH}$ and $[\mathrm{F}]$ based on a $3 \times 5$ factorial study design ( $\mathrm{pH} 4.9 ; 5.2 ; 5.5 / 0 ; 0.1 ; 0.5 ; 1.5 ; 4$ ppm F). $\Delta \mathrm{F}$ was monitored for 11 days. Subsequently, lesions were demineralised in $50 \mathrm{mM}$ acetic acid, $2.25 \mathrm{~mm} \mathrm{Ca}, 1.35 \mathrm{~mm} \mathrm{Pi}, 130 \mathrm{~mm} \mathrm{KCl}$ for two 24 -h periods with $\Delta \mathrm{F}$ being monitored. Data were analyzed using repeated measures analysis of covariance. In general, a good $\mathrm{F}$ dose-response was observed with increasing $[\mathrm{F}]$ resulting in more remineralisation regardless of $\mathrm{pH}$. Small differences in remineralisation between different $\mathrm{pH}$ values were also observed, with higher $\mathrm{pH}$ values resulting in more remineralisation, although paired with an increased sample to sample variability. After 11 days, lesions exposed to solution $\mathrm{pH} 5.5 / 4 \mathrm{ppm} \mathrm{F}$ displayed a $\Delta \Delta \mathrm{F}$ of $28.2 \pm 14.0 \%$. No remineralisation was observed in lesions exposed to F-free solutions, regardless of $\mathrm{pH}$. Remineralisation was only noticeable under the following conditions: $\mathrm{pH} 4.9$ - $[\mathrm{F}] \geq 1.5$ ppm, pH 5.2 - $[\mathrm{F}] \geq 1.5$ ppm, pH 5.5 - $[\mathrm{F}] \geq 0.5$ ppm. Subsequent demineralisation showed that only very little protection was offered by exposure to the F-containing solutions, and further demineralisation compared to baseline was observed on lesions not being remineralised initially. The present study has shown that QLF, despite its limitations, is a useful tool in studying de- and remineralisation of caries lesions under plaque fluid-like conditions. 


\section{5 \\ Commercially Available Saliva Substitutes: Effects on Subsurface Enamel Lesions in vitro}

P. Tschoppe ${ }^{a}, *$, H. Meyer-Lückel ${ }^{b}$, A.M. Kielbassa ${ }^{a}$

peter.tschoppe@charite.de

aDepartment of Operative Dentistry and Periodontology,

University School of Dental Medicine, Charité, Berlin, ${ }^{b}$ Clinic for

Conservative Dentistry and Periodontology, School for Dental

Medicine, Christian-Albrechts-University, Kiel, Germany

Saliva substitutes are often the only treatment option to relieve the symptoms of patients suffering from hyposalivation. Currently, there are numerous commercial products available; however, only scanty information on the effects on dental hard tissues is available. The aim of this study was to investigate the effects of commercially available saliva substitutes on demineralized bovine enamel in vitro. Bovine enamel specimens (n/group 9-14) were prepared and demineralized (subsurface lesions). Subsequently, specimens were stored for 5 weeks in either one of $10 \mathrm{sa}$ liva substitutes [A.S Saliva Orthana, Artisial, Glandosane, Mouth Kote, Oralube, Saliva natura, modified Saliva natura (supersaturated with respect to dicalcium phosphate dihydrate), Saseem, TMP Tüshaus, Xerostom], or a remineralizing solution (positive control) at $37^{\circ} \mathrm{C}$. Differences in mineral loss [deltadeltaZ, vol\% $\times$ $\mu \mathrm{m}$; mean (SD)] between values before (deltaZDemin) and after storage (deltaZEffect) were evaluated from microradiographs. Mouth Kote was highly erosive, and induced significantly lower deltadeltaZ values $[-46,756(5,657)]$ compared to storage in Glandosane $[-6,115(1,777)]$ or Xerostom $[-7,300(3,339)]$ ( $\mathrm{p}<$ 0.05; ANOVA, Tukey), while these in turn resulted in significantly lower deltadeltaZ values compared to all other solutions $(\mathrm{p}<$ 0.05 ). No significantly different deltadelta $Z$ values could be observed between A.S Saliva Orthana, Artisial, Oralube, Saliva natura, Saseem, and control ( $p>0.05)$. Significantly decreased deltaZEffect values [indicating a remineralization compared to baseline; deltaZDemin control: 2,113 (66), deltaZDemin modified Saliva natura: 2,101 (149)] could be observed for specimens of control [742 (758)] and modified Saliva natura [1,205 (646); $\mathrm{p}=0.011$ and $\mathrm{p}=0.033$, resp.; adjusted; adjusted paired t-test]. Within the limitations of an in vitro study it can be concluded that Glandosane, Mouth Kote as well as Xerostom reveal demineralizing properties, whereas modified Saliva natura induces remineralizing effects on bovine enamel.

\section{6 \\ Mineral Loss and Lesion Depth of Enamel and Dentine Demineralized by Different Solutions/Gels \\ B.M. Moron ${ }^{\text {a, }}$, L.P. Comar ${ }^{\text {a }}$, A. Wiegand ${ }^{\text {b }}$, H. Yu ${ }^{\text {b }}$, M.A.R. Buzalaf ${ }^{\text {, }}$, A.C. Magalhães ${ }^{a}$ \\ acm@usp.br \\ aBauru School of Dentistry, USP - University of São Paulo, \\ Bauru-SP, Brazil; b University of Zurich, Switzerland}

A wide range of protocols to induce artificial carious lesions is described in the scientific literature. Thus, the aim of this study was to analyse the characteristics of different artificial carious enamel and dentine lesions. Bovine enamel lesions $(\mathrm{n}=10)$ were induced as follows: MC gel (8\% methylcellulose gel, $0.1 \mathrm{M}$ lactic acid, pH 4.6, 14 d [ten Cate et al.: Caries Res 1996;30:400-407]); PA gel (20g/l polyacrylic acid, $0.1 \mathrm{M}$ lactic acid, $500 \mathrm{mg} / 1$ hydroxyapatite, $\mathrm{pH} 4.8$, 16 h [Iijima et al.: Caries Res 2004;38:551- 556]); MHDP (50 mM lactic acid, $3 \mathrm{~mm} \mathrm{Ca,} 3 \mathrm{~mm}$ P, methyl diphosphonate, pH 5.0, 6 d [Buskes et al.: Caries Res 1985;19:490-496]) and Buffer (50 mM acetic acid, $1.28 \mathrm{~mm} \mathrm{Ca}, 0.74 \mathrm{~mm} P$ and $0.03 \mathrm{ppm}$ F, pH 5.0, 16h [Queiroz et al.: Braz Dent J 2008;19:21-27]). Bovine dentin lesions $(\mathrm{n}=10)$ were prepared either by CMC gel l ( $2 \%$ carboxymethylcellulose gel, $0.1 \mathrm{M}$ lactic acid, $\mathrm{pH}$ 5.0, $14 \mathrm{~d}$ [Thomas et al.: J Dent 2007;35:547-551]) or Buffer (50 mM acetic acid, 2.2 mM Ca, 2.2 mM P, 0.5 ppm F, pH 4.5, 7d [Preston et al: J Dent 2007; $35: 490-495])$. Lesion depth $(\mu \mathrm{m})$ and mineral loss $(\operatorname{vol} \% \cdot \mu \mathrm{m})$ were determined by TMR. Statistical analysis was done separately for enamel and dentin by ANOVA followed by Tukey's test and unpaired t-test $(\mathrm{p}<0.05)$, respectively. In enamel, only MHDP and MC gel were significantly different; MHDP induced higher and deeper mineral loss than MC gel $(1.519 \pm 805$ vs. $731 \pm 141$ vol $\% \cdot \mu \mathrm{m}, 86 \pm 50$ vs. $36 \pm 3 \mu \mathrm{m}$, respectively). In dentin, the buffer induced higher and deeper mineral loss than CMC gel $(8.187 \pm 534$ vs. $1,468 \pm 347 \mathrm{vol} \% \cdot \mu \mathrm{m}, 262 \pm 22 \mathrm{vs} .67 \pm 8 \mu \mathrm{m}$, respectively). As the characteristic patterns of artificial lesions varied distinctly, standardized protocols to induce artificial lesions are required to allow for comparison among different studies.

Funding: FAPESP - São Paulo State Research Foundation (Process 2009/03581-9 and 2008/01472-5).

\section{7 \\ Comparative Study of Radiographical Evaluation and Morphological Evaluation of Remineralization of Carbonate Apatite \\ J. Inukai ${ }^{\mathrm{a}, *}$, H. Nakagaki ${ }^{\mathrm{b}}$, K. Kato $^{\mathrm{b}}$, M. Mukai ${ }^{\mathrm{a}}$ \\ junko@dpc.aichi-gakuin.ac.jp \\ aDepartment of Dental Hygiene, Aichi-Gakuin University Junior College, ${ }^{b}$ Department of Preventive Dentistry and Dental Public Health, School of Dentistry, Aichi Gakuin University, Nagoya, Japan}

Remineralization of dental enamel is evaluated frequently using contact microradiography. The aim of this study was to compare graphical processing results with morphological results of remineralization of carbonate apatite. Synthesized type B carbonate apatite was ground into $50-100 \mu \mathrm{m} .10 \mathrm{mg}$ of the carbonate apatite was packed in 44 nylon ring of $300 \mu \mathrm{m}$ thickness, $4 \mathrm{~mm}$ diameter and filter of $5.0 \mu \mathrm{m}$. The specimen was exposed to demineralized solutions for 2 days. These specimens were divided into 4 groups and control group, 11 were exposed to remineralized solution for 1 week (Group A), 11 were exposed to remineralized solution added with 2 ppm F for 1 week (Group B), 11 were exposed to remineralized solution for 2 weeks (Group C), 11 were exposed to remineralized solution added with 2 ppm F for 2 weeks (Group D) and 10 demineralized specimens (Control). After rinsing with distilled water, the level of remineralization was determined using contact microradiography. The data was analyzed statistically by 2-way ANOVA. The Ca:P ratios were determined 
using field emission probe microanalyzer and crystal phases were evaluated using FTIR. The results showed that the remineralization rate of Group A was 40.6\%, Group B 53.8\%, Group C 47.8\% and Group D $46.6 \%$. There was no significant difference in the length of remineralization with or without fluoridation. The $\mathrm{Ca}: \mathrm{P}$ ratio of Group A was 1.76 $\pm 0.04 \mathrm{SE}$, Group B $1.69 \pm 0.02 \mathrm{SE}$, Group C $1.70 \pm 0.02$ SE and Group D $1.70 \pm 0.02$ SE. There was no significant difference in $\mathrm{Ca}: \mathrm{P}$ ratio. However, FTIR spectra indicated the increase of OCP from apatite as the time spent on remineralization lengthened or with fluoridation. Therefore, it is suggested that OCP is precipitated first on apatite particle.

Supported by JSPS KAKENHI 20592478.

\section{8 \\ In situ Remineralization Model to Predict Dose-Response Using Low-Fluoride Dentifrices and Bovine Enamel}

A.C.B.Delbem *, R.L. Afonso, B.B. Igreja, C.F. Cantagallo, M. Danellon

adelbem@pq.cnpq.br

Araçatuba School of Dentistry, Unesp - Univ Estadual Paulista,

Araçatuba, Brazil

The aim of this study was to evaluate in situ remineralization models for dose-response establishment using low-fluoride (F) concentration dentifrices. This randomized controlled doubleblind study was performed according to the volunteers' availability and had 8 experimental periods with 7-day intervals. The volunteers $(n=10)$ utilized palatal appliances containing demineralized bovine enamel blocks. Two experimental regimens (3- and 7-day) and dentifrice treatments (placebo, 275, 550 and 1,100 $\mu \mathrm{g} \mathrm{F/g}$ ) were set. The volunteers were oriented to brush their natural teeth and the appliances, including the enamel blocks, three times a day. Demineralization depth using polarized light microscopy, surface and cross-sectional hardness (Knoop) and F concentration in enamel $\left(\mu \mathrm{g} \mathrm{F} / \mathrm{mm}^{3}\right)$ were determined. Data were homogeneous and submitted to variance analysis $(\mathrm{p}<0.05)$. Demineralization areas presented a mean depth of $47.7 \pm 12.6 \mu \mathrm{m}$ with traits of subsurface lesion. Hardness analysis showed a remineralization improvement as $\mathrm{F}$ concentration in dentifrices increased, for the 3-day experimental regimen $(\mathrm{p}<0.05)$. The augment of time and exposure to $\mathrm{F}$ dentifrices did not increase $\mathrm{F}$ concentration in enamel, considering similar $\mathrm{F}$ dentifrices ( $\mathrm{p}>0.05$ ). In the 3 -day experimental regimen, all groups presented significant differences in remineralization ( $\mathrm{p}$ $<0.05$ ). Regarding the 7-day regimen, the following groups presented similar values $(\mathrm{p}>0.05)$ : placebo $=275 ; 275=550 ; 550=$ $1,100 \mu \mathrm{g} \mathrm{F} / \mathrm{g}$. In addition, the dentifrice with $550 \mu \mathrm{g} \mathrm{F} / \mathrm{g}$, 7-day experimental period, showed similar degrees of remineralization $(\mathrm{p}$ $>0.05$ ) when compared to dentifrice with $1,100 \mu \mathrm{g} F / \mathrm{g}$ (3- and 7 -day). It may be concluded that the in situ 3-day remineralization model showed the ability to verify dose-response; however, it is more suitable for testing low $\mathrm{F}$ concentration dentifrices.

Funding: CAPES (Brazilian Coordination of Training of Higher Education Graduate).

\section{9}

\section{Reduction of Enamel Demineralisation by a Hydroxyapatite Containing Fluoride Toothpaste}

\author{
K. Hornby *, M. Evans, A. Joiner \\ kate.hornby@unilever.com \\ Unilever Oral Care, Bebington, UK
}

The objective of this study was to evaluate the enamel protective effects of a novel toothpaste formulation containing hydroxyapatite (HAP) and sodium monofluorophosphate (SMFP) versus control formulations from acid erosion in vitro. Bovine enamel specimens were prepared and baseline surface microhardness (SMH) measurements taken. Specimens were stratified into three groups $(\mathrm{n}=9)$ and mounted in dental wax. Each group was initially treated with sterilised whole human saliva for $2 \mathrm{~h}$ to form a pellicle and then underwent a $\mathrm{pH}$ cycling protocol as follows: toothpaste slurry (1:2, toothpaste:water) (3 min); $0.3 \% \mathrm{w} / \mathrm{w}$ citric acid, $\mathrm{pH} 4.0$ (2 min); saliva (60 min), with water rinses between each treatment. The cycling protocol was repeated 12 times and then the SMH was remeasured. The mean percentage change in $\mathrm{SMH}$ was calculated for each group. The toothpaste slurry treatments were either the novel toothpaste containing HAP/SMFP (A), its non-HAP control toothpaste (B) or a non-fluoride control toothpaste (C). The mean percentage change (s.d.) for each treatment group was: A 6.7 (6.9); B 15.7 (6.9), and C 28.7 (3.1). Statistical analysis (ANOVA, TukeyKramer) showed that all treatments were significantly different ( $\mathrm{p}$ $<0.05$ ) to each other. It can be concluded that the novel HAP containing toothpaste can give additional protection to enamel from acid erosion over non-HAP formulations.

Funding: Unilever Oral Care, Bebington, UK.

\section{0 \\ A Comparison of Terahertz Imaging with Transverse Microradiography for Measuring Mineral Loss in Enamel \\ D. Churchley ${ }^{\mathrm{a}, *}$, R.J.M. Lynch ${ }^{\mathrm{a}, \mathrm{b}}$, F. Lippert ${ }^{\mathrm{a}}$, L. Cooper ${ }^{\mathrm{b}}$, S.M. Higham ${ }^{\text {b }}$, J. Alton ${ }^{\mathrm{c}}$ \\ david.r.churchley@gsk.com

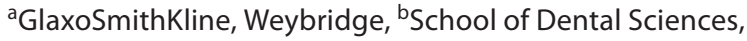 \\ University of Liverpool, Teraview Limited, Cambridge, UK}

Transverse micro-radiography (TMR) is the 'gold standard' for measuring mineral loss in enamel but is destructive in nature. The aim was to compare a non-destructive imaging modality (Terahertz Pulsed Imaging (TPI)) with TMR for measuring mineral loss in enamel. Lesions with diverse mineral characteristics were created using three demineralising systems. In the first (US), enamel bocks were immersed in lactic acid/methylcellulose gel, $\mathrm{pH} 4.6$ and incubated at $37^{\circ} \mathrm{C}$. Thirty enamel blocks were demineralised for 7, 11 and 18 days respectively. The second and third (CaPi/0.05F and $\mathrm{CaPi} / 0.10 \mathrm{~F}$ respectively) were similar but calcium $\left(4.10 \mathrm{mmol} / \mathrm{l}\right.$, as $\left.\mathrm{CaCl}_{2} \cdot 2 \mathrm{H}_{2} \mathrm{O}\right)$, phosphate $(8.00 \mathrm{mmol} / \mathrm{l}$, as $\left.\mathrm{KH}_{2} \mathrm{PO}_{4}\right)$ and fluoride $(0.05$ and $0.10 \mathrm{ppm}$, as $\mathrm{NaF}$ ) were added. In each system, thirty enamel blocks in three groups of ten were demineralised for 14, 21 and 27 days respectively. All specimens 
were imaged using a TPI imaga 1000 (TeraView Ltd). Reflection data were used to calculate lesions' refractive index (RI) profiles and the differences in the integrated areas between sound enamel and post demineralisation profiles were used to calculate $\Delta \mathrm{Z}$ $\left(\Delta \mathrm{Z}_{\mathrm{TPI}}\right.$, units au). $\Delta \mathrm{Z}$ was measured subsequently using TMR

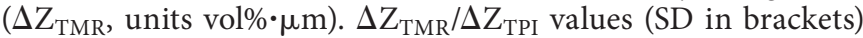
were (US) 2,732 (562)/20.2 (4.85), 4,568 (749)/42.0 (7.69) and 5,143 (588)/65.0 (3.83) for 7, 11, 18 days respectively, (CaPi/0.05) 1,925 (620)/20.8 (9.50), 2,762 (1,187)/40.3 (15.1) and 4,344 (708)/44.3 $(6.7)$ at $14,21,27$ days respectively and (CaPi/0.01) 2,802 $(1,440) / 20.7$ (11.3), 3,863 (1,400)/36.2 (12.7) and 4,041 (1,742)/43.0 $(11.5)$ at $14,21,27$ days respectively. Significant $(\mathrm{p}<0.05)$ correlations between $\Delta \mathrm{Z}_{\mathrm{TMR}}$ and $\Delta \mathrm{Z}_{\mathrm{TPI}}$ (Pearson's, all data) were observed in each group. These findings demonstrate that TPI could be used as an adjunct to TMR as a 'gold standard' technique for hard tissue imaging.

\section{1 \\ Effects of Saliva Substitutes in Combination with Fluoride Products on Bovine Dentin Subsurface Lesions}

D.L. Zandim ${ }^{\mathrm{a}, *}$, P. Tschoppe ${ }^{\text {a }}$, J.E.C. Sampaio ${ }^{\mathrm{b}}$, A.M. Kielbassa $^{\mathrm{a}}$

daniela.zandim@charite.de

aDepartment of Operative Dentistry and Periodontology,

University School of Dental Medicine, Charité, Berlin, Germany;

bSão Paulo State University - UNESP, School of Dentistry of

Araraquara, Brazil

Patients suffering from hyposalivation are instructed to use saliva substitutes to alleviate oral complaints and are encouraged to intensify their oral hygiene to prevent demineralization of dental tissues. Some saliva substitutes have been shown to demineralize dentin; this effect might be counteracted by the application of fluoride-containing products, while fluorides might increase the remineralizing effects of saliva substitutes. The purpose of this in vitro study was to evaluate the effects of two artificial salivas in combination or not with fluoride products on remineralization of bovine dentin subsurface lesions. Dentin specimens were demineralized, and stored in either Volvic mineral water (control; $\mathrm{pH}$ 7.0; Danone), Glandosane ( $\mathrm{pH}$ 5.2; Cell pharm), or modified Saliva natura (SN, saturation with respect to octacalciumphosphate $=2 ; \mathrm{pH} 5.98$; medac) for 5 weeks $\left(37^{\circ} \mathrm{C}\right)$. The following treatments were applied twice daily ( $n=12$ /group): (1) no treatment; (2) immersion in pure Elmex sensitive mouthrinse (250 ppm F-; 10 min; Gaba); (3) brushing with Duraphat toothpaste (5,000 ppm F-; Colgate)/storage solution slurry (5 s; ratio 1:3); (4) combination of treatments 2 and 3. Differences in mineral loss [deltadeltaZ, vol\% $\times \mu \mathrm{m}$; mean (SD)] before and after treatment were evaluated (transversal microradiography). Specimens stored in Glandosane showed significantly lower deltadeltaZ values $[-3,796(710)]$ compared to water [242 (361)] and Saliva natura [817 (340)] ( $\mathrm{p}<0.05$; ANOVA, Tukey). Treatment with fluoride products inhibited the demineralizing effects of Glandosane [72 (290); 5 (392); 7 (556)], while the combination of Saliva natura with daily application of both fluoride products significantly increased remineralization $[1,423(319)](\mathrm{p}<0.05)$. In conclusion, modified Saliva natura revealed remineralizing effects, and this was in- creased by simultaneous application of both fluoride products. Fluoride products inhibited the detrimental effects of Glandosane.

This study was supported in part by the Coordination for the Improvement of Higher Education Personnel/CAPES (Brasilia, DF, Brazil) and the Forschungsgemeinschaft Dental e. V. (Cologne, Germany).

\section{2 \\ Promoted Enamel Remineralization and Acid Resistance due to Fluoride Chewing Gum \\ Y. lijima ${ }^{\text {a, }}$, E. Suyamaa ${ }^{\text {b }}$, T. Tamura $^{\text {b }}$, T. Ozawa $^{\text {b }}$, A. Suzuki $^{\text {b }}$, S.lijima ${ }^{a}$ \\ iijima@nagasaki-u.ac.jp

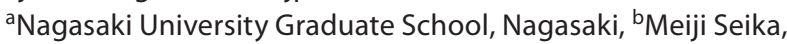 \\ Tokyo, Japan}

Low levels of fluoride ions in oral environment, especially at the saliva/enamel interface are beneficial for preventing dental caries and for remineralization of enamel. The current aim of in situ study was to evaluate enamel remineralization and acquisition of acid resistance by using sugar free chewing gum containing a green tea extract. Forty five adult volunteers attended in crossover, double blind study and wore removable intra-oral appliances with human enamel. The enamel samples were previously demineralized in $0.1 \mathrm{M}$ lactic acid conditions for 3 days. All subjects chewed tea extracts (containing $25 \mu \mathrm{g}$ fluoride/pellet) and placebo gum for 20 min 2 times daily for 4 weeks. The samples were sectioned and microradiographed (HB-50, HITEX) after exposure to acid conditions again. Remineralization and acid resistance was evaluated by mineral change value $(\Delta \mathrm{Z}$, in vol\% $\% \mu \mathrm{m})$. Mixed saliva was collected at $0,30 \mathrm{~s}, 1,3,5,10,15$, and 20 min with TISAB IV and analyzed by a fluoride ion-specific electrode (F-53/6561-10C, HORIBA Ltd., Japan). Enamel samples were dissolved in $0.5 \mathrm{M} \mathrm{HClO}_{4}$ acid and its $\mathrm{Ca}$ ion was measured by an atomic absorption spectrometer (Z-5300, HITACHI, Japan). For the calculation of acid etched depth $(\mu \mathrm{m})$ and fluoride content (ppm), a density of $2.95\left(\mathrm{~g} / \mathrm{cm}^{3}\right)$ for enamel and $37.5 \% \mathrm{cal}-$ cium concentration of enamel were used. Fluoride concentration for salivary peak height was $3.93 \pm 1.28 \mathrm{ppm}$ (mean $\pm \mathrm{SD}$ ) within $1 \mathrm{~min}$ and kept above $0.18 \pm 0.20 \mathrm{ppm}$ even after chewing for $20 \mathrm{~min}$. These higher fluoride concentration in saliva than baseline $(0.05 \pm 0.03 \mathrm{ppm})$ brought higher fluoride concentration of $712 \pm 137 \mathrm{ppm}$ at the remineralized region than $378 \pm 71 \mathrm{ppm}$ for placebo gum from the surface until $30 \mu \mathrm{m}$ depth $(\mathrm{p}<0.01)$. Mineral change values of $\Delta \Delta \mathrm{Z}$ (mean $\pm \mathrm{SD}$ ) after remineralization resulted in $467 \pm 418$ and $270 \pm 427$ for fluoride chewing gum and for the placebo gum $(\mathrm{p}<0.01)$, respectively. After acid challenge they showed 1,699 $\pm 1,194$ and 2,216 \pm 964 for fluoride chewing gum and for the placebo gum $(\mathrm{p}<0.05)$, respectively. Fluoride chewing gum produced superior level of remineralization and acid resistance to the control placebo gum. In situ results suggest that regular use of green tea extracts-containing chewing gum appears useful to promote acid resistance of remineralized enamel lesions.

Funding: research fund from Nagasaki University. 
33

\section{Microstructural Investigations of Early Caries Lesions in Natural and Polished Enamel}

R.J.M. Lynch ${ }^{\mathrm{a}, *}{ }^{*}$, F. Lippert ${ }^{\mathrm{b}}$, A. Kiesow $^{\mathrm{c}}$, A. Cismak ${ }^{\mathrm{c}}$, L. Berthold ${ }^{\mathrm{c}}$, M. Petzold ${ }^{\mathrm{C}}$

richard.j.lynch@gsk.com

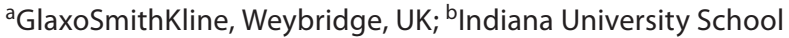
of Dentistry, Indianapolis, Ind., USA; 'Fraunhofer Institute for

Mechanics of Materials, Halle, Germany

The aim was to investigate the microstructure of early caries lesions formed in vitro in natural and polished human enamel. Specimens were prepared from unerupted third molars and demineralised using unsaturated (14 days) and partially saturated (21 days) acid-gels yielding lesions with different mineral distributions (low- and high-R). Lesions were analysed topographically using SEM and cross-sectionally using SEM, EDX and TEM after FIB-lamellae preparation. Thin sections were analysed by TMR. In general, lesions formed more homogenously and more subsurface demineralisation (lesion depth/volume) occurred in polished than in natural enamel. However, lesion surface zones were more pronounced (degree of mineralisation and width) in natural than in polished enamel. Differences in mineral distribution were observed between low- and high-R lesions using SEM and EDX and confirmed using TMR. Topographical analysis also revealed an increase in surface roughness for all demineralised specimens, but a more pronounced formation of diffusion channels/pores was observed for high- in comparison to low-R lesions. FIB-TEM crosssectional near-surface (approx. $5 \mu \mathrm{m}$ ) investigations of untreated specimens revealed a less dense, inhomogeneous crystal structure at the surface for natural than polished enamel, which can be attributed to the immature surface structure of unerupted enamel. On demineralised specimens, differences in crystal density between low- and high-R lesions were observed using FIB-TEM. Low-R lesions showed an almost intact surface zone of approx. 0.5 $\mu \mathrm{m}$, whereas a less dense crystal structure at the surface was observed for high-R lesions and irrespective of the nature of the tissue. The present study has shown that it is possible to differentiate between low- and high-R lesions formed in natural and polished enamel using microstructural analysis techniques in combination with TMR. Furthermore, differences exist in lesion formation between polished and natural enamel.

\section{4 \\ Correlations between Chemical, Optical and Mechanical Parameters on the Dentin-Enamel Junction of Molar Teeth \\ R. Chalas*, J. Nowak, T. Bachanek, A. Kuczumow \\ drrzenia@wp.pl \\ Department of Conservative Dentistry, Medical University of Lublin, and Department of Chemistry, Catholic University of Lublin, Poland}

Conventionally, enamel and dentin caries were described as two independent entities. Nowadays, changes in dentin during caries progression cannot be understood without taking the spread of the enamel lesion. The first signs of dentin reactions to the enamel lesion can be noted at the biochemical and histochemical level. Basic on actual knowledge we assume that dentinenamel junction (DEJ) is the border of reversibility for dental caries. For many years it had been thought that the dentin demineralization is spreading in a lateral direction along DEJ. Today it is interpreted as a reaction to stimuli in the direction of the rods from the less advanced parts of the enamel lesion approaching DEJ. Objective: The aim of the study was to assess the correlations between chemical, optical and mechanical parameters on the dentin-enamel junction of human molar teeth. Material and Methods: Firstly, the enamel-DEJ region was estimated and correlations between phosphorus concentration (measured by EPMA and PIXE methods) and optical signal (intensity of gray scale) on this region were assessed. Next, the chemical results were correlated with the mechanical parameter characteristic for enamel hardness. Thirdly, the dentin-DEJ region was examined and correlations between organic carbon (measured by EPMA and Raman spectroscopy methods) and optical signal were assessed. Similarly, the chemical results were compared with the mechanical parameter characteristic for dentin - friction coefficient. Results: The obtained results showed the positive correlation assessed with a Pearson test between all examined parameters. The strongest one was the correlation between phosphorus and optical signals. Conclusions: The results confirm that early microhardness changes of the outer enamel surface corresponds with chemical changes at the DEJ - boundary of this two morphologically different structures of tooth (enamel and dentin).

\section{5 \\ Measuring the Interaction between a Statherin-Like Peptide and Hydroxyapatite Using Liquid Cell Atomic Force Microscopy}

F. Ahme, A.H. Barber, J. Kosoric, P. Anderson *

p.anderson@qmul.ac.uk

Queen Mary University of London, UK

The salivary protein statherin and its shortened N-terminal analogue STN21 reduce the kinetics of enamel and hydroxyapatite (HAp) acidic demineralization. As part of an overall aim to understand the molecular mechanisms involved, the aim of this study was to measure the adhesive force between STN21 and HAp using Liquid Cell Atomic Force Microscopy (LCAFM). STN21, the $21 \mathrm{~N}-$ terminal amino acid sequence analogue of statherin was synthesized using Fmoc chemistry. HAp crystals measuring approximately $50 \mu \mathrm{m}$ in length were synthesized in a laboratory. A silicon nitride (SiN) tip used in the LCAFM was loaded with peptide by exposure to a solution containing STN21 at $1 \mathrm{mmol} \mathrm{L}-1$ (in phosphate buffer). Raman Spectroscopy was used to confirm adsorption of STN21 to the tip. The HAp crystals were attached to a silicon slide using resin and placed in the liquid cell of the LCAFM containing deionised water. Scanning Electron Microscopy in conjunction with Focused Ion Beam (FIB) milling was used to identify and locate an individual HAp crystal for use in the study. The interaction between the SiN tip and the HAp crystal face was measured with and without loaded STN21 using LCAFM. Quantification of this interaction was achieved by measuring the adhesion 
force between the SiN tip and HAp crystal face. The adhesion force between the SiN tip and the HAp crystal was $2.59 \pm 0.45 \mathrm{nN}$, reducing to $1.32 \pm 0.38 \mathrm{nN}$ when the tip was loaded with STN21. These results suggest that the N-terminal of the STN21 is bound to the SiN tip and inhibits interaction with the HAp crystal surface.

Supported by QMUL Discipline Bridging Initiative Grant Number PPA016.

\section{6}

Effect of Replenishment Rate of Demineralisation Solutions on Hydroxyapatite Dissolution Kinetics Studied Using Scanning Microradiography

H. Lingawi ${ }^{\mathrm{a}, *}$, M. Barbour ${ }^{\mathrm{b}}$, P. Anderson ${ }^{\mathrm{a}}$

h.lingawi@qmul.ac.uk

${ }^{a}$ Queen Mary University of London, ${ }^{b}$ University of Bristol, UK

The replenishment of demineralising solution adjacent to a dissolving surface has considerable influence on the rate of dissolution of solids. This is particularly pertinent to dissolution studies of enamel, and similar studies of model systems for dental caries using compressed powders of hydroxyapatite as the substrate. As part of an overall investigation of the fundamental mechanisms influencing kinetics of enamel and hydroxyapatite dissolution, the aim was to compare the dissolution rates of compressed hydroxyapatite (HAP) powder discs as a function of replenishment rate of demineralising solution, using scanning microradiography (SMR). Compressed HAP powder discs (Hitemco Medical Applications, USA) ( $\mathrm{d}=12.05 \mathrm{~mm} ; \mathrm{w}=1.25 \mathrm{~mm} ; 20 \mathrm{wt} \%$ nominal porosity) were sterilised, coated with acid-resistant varnish on all surfaces except one, preconditioned, and located in an SMR cell volume $1.96 \mathrm{~cm}^{3}$. Demineralising solution $(0.1 \%$ acetic acid buffered with $1 \mathrm{M} \mathrm{KOH}, \mathrm{pH}$ 4.0) was pumped at various replenishments rates using a variable speed circulating pump. The rate of HAP dissolution (RDHAP) was measured using SMR at a single centrally located point on each disc for periods of $24 \mathrm{~h}$ at $22^{\circ} \mathrm{C}$. Each measurement was repeated in triplicate. The mean RDHAP was: 0.823E-04, 1.18E-04, 1.70E-04, 2.47E-04, 2.84E-04, 3.13E-04, $3.31 \mathrm{E}-04 \mathrm{~g} \cdot \mathrm{cm}-2 \cdot \mathrm{h}-1$ at circulation speeds of $0,0.19,0.39,0.58,0.78$, 0.97 and $1.17 \mathrm{~cm}^{3} \cdot \mathrm{min}-1$ respectively. The RDHAP statistically significantly increased for circulation speeds up to $0.78 \mathrm{~cm}^{3} \cdot \mathrm{min}-1$, but did not change significantly at higher speeds. This study demonstrates that the solution composition in contact with a demineralising HAP surface achieved by sufficient replenishment rate, or stirring, is an important parameter in HAP dissolution studies. Diffusive transport of dissolved substrate away from the dissolving HAP surface will influence the kinetics of the process.

Supported by The Ministry of Higher Education - Kingdom of Saudi Arbia (Part of PhD Project).

\section{7 \\ Dentin Laboratorial Analysis after Indirect Pulp Treatment \\ R. Franzon ${ }^{\mathrm{a}, *}$, D.M. Dalpian ${ }^{\mathrm{a}}$, L. Casagrande ${ }^{\text {b }}$, F.B. Araujo ${ }^{\mathrm{a}}$ \\ rfranzon@bol.com.br \\ aDepartment of Pediatric Dentistry, Faculty of Dentistry, \\ Federal University of Rio Grande do Sul, Porto Alegre, \\ bSchool of Dentistry, Franciscan University Center (UNIFRA), \\ Santa Maria, RS, Brazil}

The aim of this study was to evaluate remaining carious dentin microhardness from deciduous teeth submitted to Indirect Pulp Treatment (IPT) and restored with self etching primer and composite, associated or not with calcium hydroxide capping. Deciduous molars treated with IPT and restored with Clearfil SE Bond - Kuraray, Japan (CSE) and composite Filtek-Z250 - 3M ESPE, EUA were clinically and radiographically evaluated until exfoliation. Seven teeth capped with calcium hydroxide (Dycal - Dentsply, EUA - DY) and 10 with CSE were collected and compared with 10 sound deciduous molars where 3 - to 4 -mm deep cavities were prepared, and also with 10 carious molars were partial caries removal was conducted in vitro. Microhardness analysis was performed after sample sectioning and polishing, at 10-, 35-, 60-, 85-, 110 - and $200-\mu \mathrm{m}$ depths and later each $100 \mu \mathrm{m}$ until reaching the pulpar chamber roof. The date was analysed using Kruskal-Wallis Test $(\mathrm{p}<0.05)$. No difference was observed in microhardness values between CSE and DY groups in all depths analyzed, otherwise when compared with carious group, CSE presented significantly higher values from $35 \mu \mathrm{m}$ depth, while this increase was observed only with $200 \mu \mathrm{m}$ in the DY group. From $700 \mu \mathrm{m}$ depth no difference was observed in the microhardness values between all groups in study. It can be concluded that deciduous teeth submitted to IPT presented a hardness increase suggesting mineral gain in the remaining carious tissue, independently of the capping material type, Dycal or CSE.

Supported by Federal University of Rio Grande do Sul (UFRGS).

\section{8 \\ X-Ray Microtomographic Study of the 3-Dimensional Remineralisation of Natural and Artificially Demineralised Caries Lesions \\ H.S. Wassif*, S.E.P. Dowker, G.R. Davis, J.C. Elliott \\ s.e.p.dowker@qmul.ac.uk \\ Barts and The London, Queen Mary's School of Medicine and Dentistry, University of London, UK}

The aim was to determine the feasibility of using X-ray microtomography (XMT) to assess $3 \mathrm{D}$ remineralisation in natural and artificially demineralised lesions. The laboratory XMT system provided high spatial resolution (14.96 $\mu \mathrm{m}$ voxel side-length), high contrast resolution, and kinematic repositioning of specimens. The effective monochromatic energy was $\sim 40 \mathrm{keV}$. Specimens included two molars with natural fissure caries and two quarter blocks of molar teeth in which artificial smooth surface 
lesions were produced through unvarnished windows by 30 days demineralisation ( $\mathrm{pH}$ 4.5). Changes in mineral content within each specimen were determined from measurements of linear attenuation coefficient (LAC cm-1) at baseline, and after periods of immersion in $250 \mathrm{ml}$ remineralising solution ( $\mathrm{pH} \mathrm{8)}$ to $>100$ days for natural lesions and $>150$ days for the artificial lesions. Results: In the artificial lesions, there was a trend of remineralisation with time but the extent varied within the lesions. LAC measurements increased by up to $25 \%$ after $>150$ days but did not reach the baseline value before demineralisation. By contrast, in natural lesions, there was no remineralisation detected after 100 days which could be due to the presence of organic material and proteins that block fissures. Conclusions: XMT can provide quantitative 3D mapping of mineral distribution during remineralisation in vitro. It is important to determine the extent and site of remineralisation and how it varies within the demineralised lesion.

Supported by EPSRC (Grants GR/L86050, GR/R28911), MRC (Grant G9824467) and Unilever plc.

\section{9 \\ An X-Ray Microtomographic Study of Natural White Spot Enamel Lesions \\ N.J. Cochrane ${ }^{\mathrm{a}, *}$, P. Anderson ${ }^{\mathrm{b}}$, G.R. Davis $^{\mathrm{b}}$, E.C. Reynolds ${ }^{\mathrm{a}}$ \\ n.cochrane@unimelb.edu.au \\ aOral Health Cooperative Research Centre, Melbourne Dental \\ School, Bio 21 Molecular Science and Biotechnology Institute, \\ The University of Melbourne, Victoria, Australia; ${ }^{b}$ Centre for \\ Oral Growth and Development, Barts and The London, Queen \\ Mary's School of Medicine and Dentistry, Dental Biophysics, \\ London, UK}

Aim: White spot enamel lesions (WSEL) are an early presentation of dental caries and are ideally treated by remineralisation back towards sound enamel. The aim of this study was to characterise twelve WSEL prior to attempting to remineralise them in vitro. In particular, the mineral densities across the lesion and depths from the surface to various points within the lesion were measured. Experimental Approach: Twelve molars with long buccal WSEL were collected, photographed and sectioned to produce three $500 \mu \mathrm{m}$ thick sections from each. The sections were mounted and imaged using quantitative X-ray microtomography at a $15 \mu \mathrm{m}$ voxel size [Wong et al. Arch Oral Biol 2004;49:937944]. Line profiles through the middle of each 3D image were taken using Image J and analysed to measure the mineral densities at various points across, and deep to the lesion. The depth from the surface to the various points in the lesion were measured. Results: The $95 \%$ confidence intervals for the mineral densities of enamel deep to the lesion, surface layer maximum, lesion minimum and the average across the lesion were 2.68-2.74, 2.35-2.60, $1.72-2.14$ and $2.02-2.32 \mathrm{~g} / \mathrm{cm}^{3}$ respectively. The mean distance from the surface to the point of maximum lesion density, midpoint lesion density, minimum lesion density and 95\% of sound enamel density were $72 \pm 27,157 \pm 67,288 \pm 132$ and $517 \pm 183$ $\mu \mathrm{m}$ respectively. Significant negative correlations were found between total lesion depth and minimum lesion mineral density, average lesion mineral density and depth to the point of minimum mineral density. No relationship was found between total lesion depth and maximum mineral density in the surface layer, or the distance from the surface to this point. Conclusion: The WSEL studied were highly variable and had surface layers of considerable thickness.

Supported by the Australian Dental Research Foundation Hession Award.
40
A Quantitative MicroCT Pilot Study of Acid Etching and Resin Infiltration of Proximal Enamel Lesions
A. Nyugen ${ }^{\text {a }, ~ W . ~ Z o u ~}{ }^{\text {b }}$, A. Jones ${ }^{\text {c }, ~ M . V . ~ S w a i n ~}{ }^{\mathrm{d}}$ * *
mswain@mail.usyd.edu.au

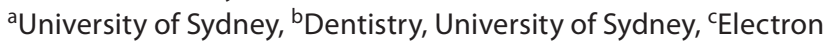
Microscopy Unit, University of Sydney, dBiomaterials Unit,
Dentistry, University of Sydney, Australia

Aim: To quantify the 'effective' mineral density changes associated with acid etching and resin infiltration of enamel caries proposed by Paris et al (JDR 86, 662, 2007) using microCT with a quantitation method developed by Huang et al. (J Dentistry 35: 737, 2007) Experimental approach: Six molar teeth with obvious natural proximal lesions recently extracted for unrelated clinical purposes were investigated. The teeth were evaluated using microCT (Skyscan, Belgium) before, after acid etching and following resin infiltration using a kit developed by DMG (Hamburg, Germany). Calibration standards of hydroxyapatite with different mineral density were incorporated with the teeth for all microCT scans to enable quantitative analysis. Results: MicroCT grey level images incorporating the calibration standards enabled mineral density contours through each of the enamel lesions to be generated. The pre-treated lesions ranged in depth from 250 to $800 \mu \mathrm{m}$ and typically showed 30 to $50 \%$ reduction in mineral density compared with adjacent sound enamel. All teeth showed a significant further reduction in the mineral density (12 to $21 \%$ ) through the lesion following acid etching and a minor increase (6 to $12 \%$ ) in mineral density after resin infiltration. With a number of the teeth the acid etching procedure did not completely dissolve the outer hard layer on the lesion. Conclusions: MicroCT enables the changes in mineral density of enamel proximal lesions to be quantified at the different stages of the treatment protocol proposed by Paris et al (2007).

Supported by DMG Hamburg (reparative kit).

\section{1 \\ Visual Assimilation of Artificial Enamel Caries Lesions by Infiltration in vitro
S. Paris, J. Keltsch *, C.E. Dörfer, H. Meyer-Lueckel
jasper.keltsch@gmx.net
Clinic for Operative Dentistry and Periodontology, School
of Dental Medicine, Christian-Albrechts-Universität zu Kiel,
Germany

Infiltration of caries lesions with light-curing resins is efficacious to inhibit further lesion progression. As a positive 'side effect' the whitish appearance of caries lesions is altered by the pen- 
etrating resin due to reduction of light scattering. The aim of this in vitro study was to assess the effect of resin infiltration on the visual appearance of artificial caries lesions. In each of 20 bovine enamel specimens two artificial lesions were created ( $\mathrm{pH} 4.95,50$ days), leaving two sound windows of enamel. After etching $(37 \%$ $\mathrm{H}_{3} \mathrm{PO}_{4}, 3 \mathrm{~s}$ ), in each specimen one lesion and one sound window were infiltrated (Icon; DMG). The other (untreated) lesion and sound window served as negative and positive controls, respectively. Half of the four areas of each specimen was polished (removal $\sim 15 \mu \mathrm{m}$ ). Specimens were remineralized ( $\mathrm{pH} 7.0$ ) for 25 days. During that period specimens were additionally stored in a coffee/red wine mixture (1:1) for 10 min daily and brushed with fluoride toothpaste $(1,400 \mathrm{ppm}) 12 \mathrm{~h}$ later. After infiltration as well as after the remineralization period standardized photographs were taken and $\mathrm{L}^{*} \mathrm{a}^{*} \mathrm{~b}^{*}$-color values were measured (Photoshop CS4). Color differences compared to positive controls $(\Delta \mathrm{E})$ were calculated for each area. After infiltration, $\Delta \mathrm{E}$-values [median (Q25/Q75)] of infiltrated lesion [7 (6/10)] and infiltrated sound areas [4(3/6)] were significantly lower compared with those of negative controls [20 (17/22)] ( $p<0.001$; Wilcoxon). After remineralization, infiltrated polished lesion areas [6 (5/8)] showed significantly lower $\Delta \mathrm{E}$-values compared with (unpolished) negative controls $[11(6 / 15)](\mathrm{p}<0.05)$. Compared to remineralization alone, infiltration in combination with remineralization resulted in better assimilation of the appearance of artificial enamel lesions to adjacent sound enamel.

Supported by the Deutsche Forschungsgemeinschaft (PA1508/1-2)

\section{2 \\ Impact Evaluation on Caries-Risk Status of 5-Year- Old Vulnerable Children after Three-Year Fluoride Varnish Program Exposure \\ J. Véronneau ${ }^{\mathrm{a}}$, J.R. Lamarre ${ }^{\mathrm{b}, *}$ \\ jacques.veronneau@mcgill.ca \\ aMcGill University, and ${ }^{b}$ Agence de la Santé et des Services \\ Sociaux de Montreal, Montreal, Canada}

There is limited information concerning longitudinal topical fluoride programs as a potential mediator of early childhood caries-risk status. The aim of this study was to use a randomised control trial (RCT) initiated in 2-year-olds testing the effectiveness of fluoride varnish and to assess the impact on risk status after a 43-month follow-up period. The RCT used the scientific Consort concepts leading randomly to the allocation of two experimental groups to a homogeneous distribution of potential bias factors. All RCT dyads (mother-child) were originally selected based on their child-stimulation attendance program or the low annual family income. Seven dental hygienists were trained and calibrated to standardise the screening procedure. The endpoint risk evaluation was based on Quebec schoolchildren program criteria defining high caries-risk category at 5 years of age as the experience of at least one smooth surface with obvious dentinal carious lesions, filling or missing for caries or with two posterior smooth surfaces with the same type of caries experience. Risk status was performed using mirrorlight, prebrushing proce- dure and $2 \times 2$ cotton for drying. A sample of 265 children (mean age 32 months) was recruited and randomly allocated in a test group of 141 children - exposed to seven biannual topical fluoride applications during the follow-up of 43 months - and 121 children in a control group exposed to normal care. At the end of the RCT, all 76-month-old children were screened using the provincial criteria. The mean proportion of children without experience $(\mathrm{dmfs})$ of caries at dentinal level was $35.1 \%$ for all of the sample. The proportion of high-risk children was $34.5 \%$ in the test group and $21.4 \%$ in the control group. In conclusion, an early fluoride varnish program to vulnerable children may not change their expected caries-risk status.

Funding by the Health Ministry, Government of Quebec, Canada.

\section{3 \\ Effect of Hydrogel Concentration and Calcium Activity on Demineralisation \\ G.J. Owens ${ }^{\text {a, }}{ }^{*}$, R.J.M. Lynch ${ }^{\text {a, b }}$, L. Cooper ${ }^{\text {a }, ~ S . P . ~ V a l a p p i l ~}{ }^{\text {, }}$ S.M. Higham ${ }^{\text {a }}$ \\ G.Owens@liverpool.ac.uk \\ ${ }^{a} S c h o o l ~ o f$ Dental Sciences, University of Liverpool, \\ ${ }^{\mathrm{b}}$ GlaxoSmithKline, Weybridge, UK}

Caries-like lesions can be developed in vitro though model systems which often incorporate a hydrated gelatinous phase in reflection of the acidic stagnation area of cariogenic plaque. Elevated calcium activity in these areas can reduce demineralisation. Hence both calcium activity $(\{\mathrm{Ca}\})$ and methylcellulose $(\mathrm{MeC})$ density may have a significant impact on the ionic activity within this phase and thus enamel demineralisation. Model demineralising systems were constructed as lactic acid ( $\mathrm{pH} 4.6) / \mathrm{MeC}$ gels (MeC concentrations 4, 8 and $12 \%$ ). MeC concentrations were investigated in triplicates and repeated for added $\mathrm{CaCl}_{2}$ concentrations ([Ca])of 10, 1, 0.1 and $0 \mathrm{mM}$. At each $\mathrm{CaCl}_{2} /$ hydrogel concentration combination, enamel demineralisation was evaluated through bovine enamel blocks immersed in a subset of the lactic acid/MeC gels. The study was conducted for a period of 14 days after which Ca ionic activities were quantified via calcium-specific electrode measurement. Integrated mineral loss (deltaZ), lesion depth (LD) and average mineral-loss (R) were measured subsequently using transverse microradiography (TMR). Mean $\{\mathrm{Ca}\}$ increased significantly with increasing [Ca] as expected; however, curve escalation coefficients were proportionally greater for higher gel densities. $\{\mathrm{Ca}\}$ was significantly reduced to approximately $10 \%$ of $[\mathrm{Ca}]$. deltaZ decreased with increasing $\{\mathrm{Ca}\}$. Lesion character changed from sub-surface for lower $\{\mathrm{Ca}\}$ to surface-softening at higher $\{\mathrm{Ca}\}$. $\mathrm{R}$ values also decreased significantly with increasing $\{\mathrm{Ca}\}$. Hydrogel density afforded ionic stagnation therefore contributing to lesion development. A relationship of increasing $\{\mathrm{Ca}\}$ with increasing $\mathrm{MeC}$ density was observed. Concluding, both $\{\mathrm{Ca}\}$ and hydrogel density can influence enamel demineralisation both quantitatively and qualitatively.

Supported by GlaxoSmithKline (Funded PhD Studentship GlaxoSmithKline), Weybridge, UK and School of Dental Sciences, University of Liverpool, UK. 


\section{4}

\section{Pre-Clinical Biofilm Model for Testing Anticariogenic} Potential of Dental Therapies

M.S. Cenci *, F.H. van de Sande, M.S. Azevedo, R.G. Lund

m.s.cenci@pq.cnpq.br

School of Dentistry, Federal University of Pelotas, Brazil

The aims of this study were to evaluate enamel demineralization response to cariogenic conditions induced by several sucrose regimens in microcosm biofilms, and after setting the nutritional condition, to test the model with a dose-response evaluation to chlorhexidine. The microcosm biofilms from human saliva were grown on bovine enamel discs in multi-well plates fed with artificial saliva which was supplemented with several sucrose concentrations. Sucrose exposure occurred all day (batch) or in a semidynamic scheme (6 h a day). Data from acidogenicity of biofilms were collected as $\mathrm{pH}$ readings from medium supernatants and the percentual surface microhardness change (\%SMHC) was obtained by enamel surface microhardness readings. The results from sucrose regimens indicated that acidogenicity of biofilms affected enamel surface microhardness. Low sucrose exposures (batch $0.075 \%$ ) did not cause any enamel hardness change. Exposures to $0.15 \%$ sucrose were time-dependent, as enamel surface experienced mineral loss in batch but not in semi-dynamic fed. Higher sucrose concentrations $(0.5 \%$ and $1 \%)$ were just evaluated for semi-dynamic fed, and both caused significant mineral loss. The dose-response evaluation was taken in one regimen (semidynamic $1 \%$ sucrose) and both biofilm acidity and enamel mineral loss were dose-responsive to chlorhexidine treatments. In conclusion, the biofilm model assessed promoted dose-response effect to chlorhexidine and to different sucrose feeding protocols, being suitable as a pre-clinical model for testing the anticariogenic potential of treatments.

Supported by CAPES (Prodoc 420030/0-0). 


\section{Caries Research}

\section{Session 3 Clinical Studies I}

\author{
45 \\ Restorative Treatment Decisions on Occlusal Caries \\ in Norway \\ S. Vidnes-Kopperud * A.B. Tveit, I. Espelid \\ simen.vidnes-kopperud@odont.uio.no \\ Faculty of Dentistry, University of Oslo, Oslo, Norway
}

The aim was to describe variations in the threshold for operative treatment of occlusal caries in permanent teeth, use of dental materials and comparison of results from a similar study conducted in Norway in 1995-96 [Espelid et al.: Acta Odontol Scand 2001;59: 21-27]. Materials and Methods: An electronically, pre-coded questionnaire was sent in March 2009 to all dentists with e-mail address in the member register of the Norwegian Dental Association (NDA). The questions were related to caries, treatment strategies and choice of restorative materials. Of the 4,317 members of the NDA, 3,654 e-mail addresses were known and replies were obtained from $68.2 \%$ after two reminders. Significantly fewer dentists would restore lesions confined to enamel based on clinical and radiographic appearance in 2009 compared to 1995 (12.7 vs. 17. 8\%, p < $0.01)$. Most dentists preferred to drill only the carious part of the fissure rather than the whole fissure both in 2009 (78.9\%) and 1995 $(68.4 \%)(\mathrm{p}<0.01)$. In 2009, 90.1\% preferred composite as the restorative material of choice, compared to $39.1 \%$ in 1995 . The corresponding values for conventional glass ionomer cement (GIC) were 2.8 vs. $10.5 \%$, resin modified GIC 1.8 vs. $11.9 \%$, and a combination of GIC and composite: 1.6 vs. $16.1 \%$. In 2009 compomer was preferred by $1.5 \%$ of the respondents while $2.3 \%$ did not specify which material they preferred. More dentist wait until lesions are recorded in dentin before initiating operative treatment of occlusal caries in 2009 compared to 1995 . Dentists appear to be more reluctant to remove tooth substance in 2009. Composite is the dominating material of choice in 2009, and seems to have substituted both amalgam and glass ionomer cement as filling material in Class I restorations.

The study was funded by the University of Oslo.

\author{
46 \\ Resin Infiltration of Caries Lesions: An Efficacy \\ Randomized Trial \\ S. Paris ${ }^{a, *}$, W. Hopfenmuller ${ }^{\text {b }}$, H. Meyer-Lueckel ${ }^{\text {a }}$ \\ paris@konspar.uni-kiel.de \\ ${ }^{\mathrm{a} C l i n i c}$ for Operative Dentistry and Periodontology, School \\ of Dental Medicine, Christian-Albrechts-Universität Kiel, \\ ${ }^{b}$ Department of Medical Informatics, Biometry, and \\ Epidemiology, CharitéCentrum 4, Institute of Medical Biometry \\ and Clinical Epidemiology, Charité, Berlin, Germany
}

Caries infiltration is a micro-invasive therapeutic approach to arrest progression of caries lesions. The aim of this randomized split-mouth placebo-controlled clinical trial was to assess, whether resin infiltration of proximal lesions in combination with self-applied non-operative measures is more efficacious than non-operative measures alone with respect to inhibition of caries progression. In 22 young adults, 29 pairs of interproximal lesions radiographically extending into inner half of enamel (E2) or outer third of dentin (D1) were randomly allocated to either one of two treatments. Test lesions were infiltrated (ICON, pre-product; DMG). A placebo treatment was performed in control lesions. All subjects received instructions for non-cariogenic diet, flossing and fluoridation. The primary outcome was radiographic lesion progression (digital subtraction radiography). At baseline $32 \%$ of participants had a low, $36 \%$ a moderate, $23 \%$ an increased, and $9 \%$ a high caries risk (Cariogram 2.01). After 18 months, 28 lesion pairs could be re-evaluated radiographically using standardized bitewing holders (pre-product; DMG). No unwanted effects could be observed. Radiographic progression was recorded in $3 / 28$ test lesions (11\%) and 11/28 control lesions (39\%) ( $\mathrm{p}<0.008$; McNemar test). For interproximal caries lesions extending radiographically around the enamel-dentin junction (E2, D1) resin infiltration in combination with self-applied non-operative measures is more efficacious in reducing lesion progression compared with self-applied non-operative measures alone.

Supported by DMG, Hamburg and conducted at Charité Universitätsmedizin Berlin. 


\section{7 \\ The Evaluation of Resin Infiltration for Masking Labial Enamel White Spot Lesions}

S. Kim * J.H. Shin, E.Y. Kim, S.Y. Lee, S.G. Yoo

shinkim@pusan.ac.kr

Pusan National University, School of Dentistry, Yangsan, Korea

The aim of this study was to clinically assess the effectiveness of masking white spot enamel lesions with resin infiltration technique which was recently developed to arrest the incipient caries in a minimum invasive concept. Twenty teeth with DDE (Developmental Defect of Enamel) from twelve children and eighteen teeth with POD (Post-orthiodontic Decalcification) from nine children were selected and treated with resin infiltration. The clinical procedure was composed of erosion with HCL, desiccation with ethanol and application of low-viscosity light cured infiltrant resin. The standardized photos were taken before, immediately after and one week after treatment, analyzed with image analyzing software calculating $\Delta \mathrm{E}$ value. The results were classified into 3 groups; completely masked; partially masked; not changed. Among twenty teeth with DDE, five teeth (25\%) were classified as completely masked whereas seven (35\%) and eight teeth (40\%) showed partially masked and not changed respectively. Among eighteen teeth with POD, eleven teeth (61\%) showed completely masked, six teeth $(33 \%)$ partially masked and one tooth $(6 \%)$ not changed. In some teeth, the result was more improved after 1 week than immediately after infiltration. Conclusively, masking effect was dramatic in some cases, while totally or partially not in other cases. It was thought that the effect depends on lesion depths; namely, in the lesions deeper than penetration range of infiltrant resin, the masking effect was a little or none. The method for clinically measuring lesion depths was thought highly desirable to distinguish the indication of resin infiltration for masking purpose.

Materials were kindly supplied by DMG-Germany.

\section{8 \\ 1-Year Radiographic Results of Infiltrating, Sealing and Giving Flossing Instructions: Proximal Lesions in Young Adults \\ S. Martignon ${ }^{\mathrm{a}, *},{ }^{*}$.R. Ekstrand $^{\mathrm{b}}$ \\ smartignon@gmail.com \\ aUniversidad El Bosque, Bogotá, Colombia; ' ${ }^{\text {University of }}$ \\ Copenhagen, Denmark}

The aim of this split-mouth design study was to assess the efficacy of resin infiltration of proximal carious lesions on young adults. Positive controls were sealed lesions and negative controls were flossing instructions. In each of 40 patients, three proximal lesions with radiographic extension around the enamel-dentinjunction (E2) or in the dentine outer-third (D1) were selected. Lesions were randomly allocated to one of three treatment modalities and treated after $48 \mathrm{~h}$ elective-temporary separation: A) Infiltration (test lesion): 15\%-HCl gel etching and light-cured resin; $\mathrm{B}$ ) Sealing with an adhesive (positive control); and C) placebo-treat- ment (negative control). All subjects received instructions for flossing. Standardized x-rays were obtained at baseline and after 1 year. They were analyzed by an examiner blind to the study and to group using pair-wise recording of the radiographs and by digital-subtraction radiography. At baseline subjects' caries risk was distributed as follows: 45\%-low; 30\%-moderate, and 25\%-high. The 12-months follow-up examinations were conducted in $97.5 \%$ of the sample $(\mathrm{n}=39)$. No unwanted effects such as pain, vitality loss or staining was noted after 1 year. Pair-wise radiographic comparison showed progression in seven of the infiltrated, in 12 of the sealed, and in 18 of the negative lesions. Correspondent figures for the subtraction comparison were: 17,25 , and 32, respectively. Using Mc Nemar's test significant differences were observed in lesion progression both with the pair-wise and subtraction reading methods between Infiltration and negative controls $\left(\chi^{2}=9.30 ; \chi^{2}=11.52 ; p<0.01\right)$, respectively. No significant difference was noted between Infiltration and Sealing (positive controls) ( $\mathrm{p}$ values $>0.05$ ), and between Sealing and negative controls ( $p$ values $>0.05$ ). Conclusion: After one year the infiltration of proximal caries was more efficacious than sealing lesions, which was more efficacious than flossing instructions.

We gratefully acknowledge DMG-Germany for partially funding the study.

\section{9 \\ Clinical Applicability and Safety of Resin Infiltration of Proximal Caries}

\author{
M. Alkilzy*, C. Splieth \\ alkilzy@hotmail.com \\ Department of Preventive and Pediatric Dentistry, University of \\ Greifswald, Germany
}

The aim of this prospective practice-based study is to evaluate the applicability and clinical safety of a new minimally invasive treatment for proximal initial carious lesions by infiltration. Five dentists in the Department of Preventive and Pediatric Dentistry, University of Greifswald applied the infiltration material ICON ${ }^{\circledR}$ (DMG, D) on proximal initial lesions (D1-D3 without cavitation on standardized X-ray) in primary and permanent teeth in 20 patients ( $9 \mathrm{~m}, 11 \mathrm{f}$; mean age $18.5 \pm 8.2$ years). The applicability was evaluated using two questionnaires filled out by dentists and patients. The dentist's questionnaire evaluated the medical history, caries experience and risk, time and difficulties of application, involvement of neighboring teeth and the comparison to a composite filling. The patient questionnaire evaluated the satisfaction with the procedure regarding time, comfort and complexity. At the recall after one week, the infiltrated surfaces were assessed for discoloration, marginal adaptation, gingival status and plaque accumulation. The recall ended with an oral hygiene instruction including flossing and topical fluoride application. The results showed good patient's satisfaction with the procedure and time (mean time $24.3 \pm 4.95 \mathrm{~min}$ ) which included rubber dam application (mean time $9 \pm 2.8 \mathrm{~min}$ ) and infiltration (mean time $15.3 \pm 4.4 \mathrm{~min}$ ). In four patients, the proximal spaces were insufficient due to difficulties with the separation of the teeth, which 
prolonged the infiltration time. In most cases, the dentists reported that infiltration was comparable to the application of a composite filling. At recalls, all infiltrated surfaces showed smooth transition to enamel, no discoloration, and no undesirable inflammatory or allergic signs in the adjacent gingiva. In conclusion, the infiltration of carious initial lesions may provide a simple, minimally invasive procedure for treatment of proximal initial caries.

Supported by Stiftung Innovative Zahnmedizin, Switzerland.

\section{0 \\ Treatment of Proximal Superficial Caries Lesions on Primary Molar Teeth with Resin Infiltration and Duraphat versus Duraphat only: Efficacy after One Year}

A. Bakhshandeh ${ }^{\mathrm{a}, *}$, K.R. Ekstrand ${ }^{\mathrm{a}}$, S. Martignon ${ }^{\mathrm{b}}$

kim@odont.ku.dk

aUniversity of Copenhagen, Denmark; 'bniversidad El Bosque,

Bogota, Colombia

This split mouth study aimed to assess the efficacy of resin infiltrated lesions covered by Duraphat versus Duraphat treatment only of proximal carious lesions on deciduous molar teeth. The duration of the study was one year. Inclusion criteria were children with 2 or more proximal enamel or early dentinal lesions on deciduous molar teeth detected on BW. After written consent from parents, two lesions on a total of 48 children were randomly allocated to one of the two treatment regimes: resin infiltration followed by treatment with fluoride varnish $(2.26 \% \mathrm{~F})$ (test lesion) versus only fluoride varnish (control lesion). Duraphat was applied again to both the test and control lesions 6 and 12 months after the first treatment. After one year the children were re-examined clinically and radiographically. Adequate radiographs were obtained for 39 children (81\%). One external examiner not familiar with the study scored the radiographs twice (weighted kappa value 0.88). Baseline mean age of the children was 7.17 (SD 0.68 ) and mean def-s was 8.1 (SD 6.9). Radiographically, 23\% of the test lesions had progressed versus $62 \%$ of the control lesions $(\mathrm{p}<0.01)$. Relevant data from those participants where we have no radiographical one year data did not differ from corresponding data on those who remain in the study. Thus, the therapeutic effect of both resin infiltration/fluoride varnish over fluoride varnish treatment alone was $38 \%$ and significant. To conclude: Resin infiltration in conjunction with fluoride varnish seems promising for controlling lesion progression in proximal lesions on deciduous molar.

Supported by DMG Dental-Material Gesellschaft mbH (Hamburg, Germany; material and financial aid). The Public Dental Service in Nuuk, Greenland is thanked for their kindness, understanding and letting us work on the clinics.

\section{1 \\ Which Materials Could Be Recommended for Fissure Sealing? - Results from a Systematic Review}

\author{
J. Kühnisch ${ }^{\mathrm{a}, *}$, R. Heinrich-Weltzien ${ }^{\mathrm{b}}$, R. Hickel $^{\mathrm{a}}$ \\ jkuehn@dent.med.uni-muenchen.de \\ aDepartment of Conservative Dentistry and Periodontology, \\ Ludwig-Maximilians-University of Munich, ${ }^{\mathrm{b}}$ Department \\ of Preventive and Paediatric Dentistry, University of Jena, \\ Germany
}

The aim of this systematic review of literature was to analyse the reported retention rates from clinical studies on pit and fissure sealing in relation to the used sealant material and observation time as well as to provide recommendations for the dental practice. The literature search covered the MEDLINE and EMBASE databases for the time period up to the end of 2008. After reviewing the identified abstracts $(n=1,851), 473$ clinically based publications were analysed in detail. In result of this evaluation step 140 publications included information about sealant retention with a minimum observation time of two years. All these studies were analysed for the retention of the used material(s) (UV-light-, visible-light- and auto-polymerizing sealants, fluoride-releasing materials, compomers, flowables and glass-ionomer-cements) in relation to the observation time. In result, the descriptive analyses showed that auto-polymerizing sealants have the longest clinical experience with up to 20 years observation time and a corresponding rate of $65.4 \%$ fully retained sealants. When viewing the overall retention rates then methacrylatebased materials (visible-light and auto-polymerizing sealants, fluoride-releasing products and flowables) showed similar retention rates with up to $\sim 80 \%$ fully retained sealants after two years. Contrary to this finding a remarkable retention loss was documented for UV-light-polymerizing materials, compomers and glass-ionomer-based sealants within the first two years after application. Therefore, retention rates for these materials have to be characterised as inferior. In conclusion of this systematically search of literature visible-light- and auto-polymerizing sealants, fluoride-releasing materials and flowables can be recommended for the clinical use. Bearing in mind the faster and less errorprone clinical application technique of light-polymerizing materials, therefore, these materials should be favoured in the daily dental practice.

\section{2}

Sealing Dentinal Caries Lesions in Permanent Teeth: Pilot Study

D. Hesse *, T.S. Teixeira, C.C. Bonifácio, D.P. Raggio, M.M. Braga J.C.P. Imparato

danielar@usp.br

University of Sao Paulo, Brazil

The aim of this pilot in vivo study was to verify the effect of pit and fissure sealants in dentinal caries lesions compared to conventional restorative treatment. Thirty three permanent teeth from twenty four patients had been selected and randomly allo- 
cated in two groups: G1 - sealant application and G2 - restoration with composite resin, after outer dentine removal. After treatment, patients were submitted to clinical and radiographic (bitewing) evaluation after 6 and 12 months. The scores for clinical assessment were: partial loss, total loss or total retention. In radiographic method, teeth were classified in absence or presence of caries progression. Chi-square test was performed in each period $(p<0.05)$. There was no statistic difference between groups, regarding clinical evaluation. After 6 months there was $100 \%$ total retention in both groups $(\mathrm{p}=0.727)$. After 12 months there was $92.85 \%$ total retention and $7.15 \%$ partial loss in G1 and $100 \%$ total retention in G2 $(p=0.863)$. In both groups, all cases showed absence of signs for caries progression in the radiographic exam for both periods. We conclude in this pilot study that pit and fissures sealant is more conservative treatment then conventional restoration, reducing the progress of dentinal caries lesions and preserving dental structure. Longer follow-up is necessary to confirm these findings.

Acknowledgement to University of Sao Paulo.

\section{3 \\ Clinical Evaluation of Non-Restorative Cavity Treatment (NRCT)}

\author{
R.J.M. Gruythuysen ${ }^{\mathrm{a}, *}$, W.H. van Palenstijn Helderman ${ }^{\text {b, }}$ \\ C. van Loveren ${ }^{\text {a }}$ \\ r.gruyt@planet.nl \\ ${ }^{a}$ ACTA Amsterdam, and bUMCN Nijmegen, The Netherlands
}

The present retrospective study aims to investigate extending lifetime of cavitated primary teeth by a Non-Restorative Cavity Treatment. This study estimates the clinical results of caries management. NRCT is a child friendly treatment approach consisting of: 1) making the lesions accessible for the toothbrush by slicing 2) improving self care by motivational interviewing 3) application of fluoride varnish and/or if needed for protection of the pulp, application of RMGIC lining cement (Vitrebond/3MESPE). Teeth selection: 35 approximally cavitated primary molars from $18 \mathrm{anx}-$ ious/non-cooperative children (mean age: 78.1 months, s.d. 27.9), referred for dental treatment to ACTA. During follow-up (median: 12 months) NRCT's took place at recall visits of which the frequency was based on the caries activity. Criteria for caries activity (assessed by photography and recorded information on dental charts) were: not disturbed plaque present/dentin: soft to leathery/gum: red and/or swollen. At the start, 24 of 35 teeth showed $1 / 3$ or less remaining dentin thickness. Radiographs were made when needed for clinical purposes. Criteria for failure were: the presence of irreversible pulp pathology. Criterion for restorative treatment was: reflexive hypersensibility. When caries was arrested and the cooperation was good, restorations were performed if needed for the comfort of the patient. NRCT failed in two teeth (12/22 months). After 8 months in two hypersensitive teeth a well accepted pulpotomy was performed. At the end of the follow-up time 14 caries lesions were inactive and the remaining 17 showed still activity but without pain or discomfort. NRCT was well accepted by all children. In conclusion Non-Restorative Caries Treatment seems to be a feasible approach for (temporary) dental treatment of primary teeth in anxious/non-cooperative children.

\section{4 \\ Value of Bitewing Radiographs for Detecting Approximal Caries in 6-Year-Old Dutch Children}

J.H.G. Poorterman *, J.H. Vermaire, J. Hoogstraten

j.poorterman@acta.nl

ACTA, Department of Social Dentistry and Behavioural Sciences, Amsterdam, The Netherlands

Background: When diagnosing caries using clinical judgment only, the prevalence of approximal caries is highly underestimated. However, surveys on this topic predominantly included adolescents and young adults. Aim: The aim of the study was to determine the additional diagnostic value of bitewing radiographs in 6-year-old children to detect approximal dentin caries in the primary dentition. Design: Participants were 6-year-old (regular) patients of two general dental practices with affinity for pediatric dentistry in several large communities in the Netherlands. A total of 197 patients were clinically examined (dmfs, oral hygiene) by two experienced dentists. All were considered for participation in this study, but 147 were excluded as they previously had radiographs taken at 5 years of age. Thus, the remaining sample consisted of 50 children. Interobserver agreement was calculated using Cohen's Kappa and proved to be very good for both clinical assessment $(\kappa=0.94)$ as well as radiographic assessment $(\kappa=$ 0.87). Results: In 9 patients (18\%) it was impossible to make radiographs. Bitewing radiography appeared to have an additional effect of $97 \%$ when only caries in dentin is considered. The additional value for detecting inadequate restorations was $600 \%$. Furthermore, the dmfs was highly correlated to the amount of plaque found. Conclusion: Although not possible to achieve in all 6-yearold children, bitewing radiographs can reveal a considerable amount of carious surfaces and inadequate restorations, which appear clinically sound.

\section{5 \\ Clinical Evaluation of a Fluorescent Camera for Caries Discrimination \\ E. Terrer ${ }^{\text {a }}$, A. Raskin ${ }^{\text {a }}$, B. Pelissier ${ }^{\text {b }}$, B. Levallois ${ }^{\text {b }}$, S. Longhi ${ }^{\text {, }}$, H. Tassery ${ }^{\mathrm{a}, *}$ \\ herve.tassery@numericable.fr \\ aMarseille Dental School, Marseille, ${ }^{\mathrm{b}}$ Montpellier Dental School, Montpellier, 'CNRS, Univerité Aix Marseille, Marseille, France}

The aims of the following series of experiments were to evaluate the sensitivity, specificity, positive and negative predictive values of a fluorescent camera for occlusal caries diagnosis versus visual, digital radio and laser inspection and to compare the in vivo variation of brightness of sound dentin, active and arrested carious dentin illuminated with the same device. Kinetic Polymerase Chain Reaction was also used to correlate the fluorescence with the bacterial level. Methods: Part 1: 15 patients were randomly selected and 16 teeth (molars and premolars per patient were independently analyzed by 2 observers. Using both ICDAS classification, observer 1 provided visual inspection and bitewing radiography and observer 2 illuminated the same teeth using the fluorescent camera and laser device. Laser device was used as gold 
standard. Part 2: In vivo, 15 treated carious teeth were illuminated with the fluorescent camera and pictures were recorded. To analyze the brightness in RGB space, Image software was used and a short constant rectangular section was drawn passing through the healthy area and the pathologic area to reveal the dynamic brightness profile. Results: Part 1: Laser device used as standard. Respectively Fluorescent camera, Digital radio, Visual inspection: sensibility $(0.644,0,0.266)$, specificity $(0.820,0.987$, 0.883), PPV (0.674, 0, 0.571), NPV $(0.8,0.631,0.673)$. Part 2: Statistically significant differences in the brightness and dynamic brightness profile variations were found between the different areas respectively and between active and arrested caries processes. Kinetic Polymerase Chain Reaction results and fluorescence were positively correlated. In conclusion we found that the fluorescent camera revealed a good specificity and the highest sensitivity and that in restorative procedure the device could be useful for caries discrimination activity.

Funding: Acteon-Sopro industry.

\section{6}

\section{Salivary Flow Rate, Buffer Capacity, Microbial Counts and Caries Activity of Young Healthy Subjects}

D. Karaiasheva, M. Marinova, E. Boteva *

e_boteva@abv.bg

Faculty of Dental Medicine, Sofia, Bulgaria

The aim of the present study was to investigate the relationship between diet, salivary flow rate, buffer capacity, microbial counts and DMFT, and differences in two groups of caries active and caries resistant healthy subjects. All volunteers were without open caries cavities. The age range of the volunteers ( $\mathrm{n}=44,20$ males and 24 females) was 18-25 years. Dietary data was investigated with questionnaire filled with personal interview. Salivary qualities, S. mutans and Lactobacilli counts were tested in two regimes - hygienic and nonhygienic for $24 \mathrm{~h}$. Tests were performed in the morning, in same hours and non-stimulated saliva was collected, tested with Vivacare Line-CRT (Ivoclar, Vivadent). The results were compared caries active versus caries resistant and male versus female subjects. The statistical analysis was performed using nonparametric tests, Mann Whitney and Fisher's t-test. Significant differences $\mathrm{p}<0.05$ were found in the salivary flow rate and microbial counts of $S$. mutans and Lactobaccilli, between caries active and resistant groups in normal - 3.35-2.95 $\mathrm{ml} / 5 \mathrm{~min}$ and nonhygienic $-2.65-2.70 \mathrm{ml} / 5 \mathrm{~min}$, regimes. The microbial counts of the two groups in the two tested regimes, were defined as high risk when higher than $10^{5}$ and low risk when lower than $10^{5} \mathrm{CFU} / \mathrm{ml}$ although plaque was not accumulated at all for this small duration of time. Microbial counts among caries active group were up to $10^{7} \mathrm{CFU} / \mathrm{ml}$ among female and $10^{9} \mathrm{CFU} /$ $\mathrm{ml}$ among male subjects in nonhygienic regime. Conclusions were related to the permanent quality of dental biofilm in caries active subjects, and to the related to diet possible postresorbtional effects on saliva and de- and re-mineralization qualities of mineralized oral structures.

Funding: Council of medical sciences, Medical University, Sofia, Bulgaria.

\section{7}

Oral Hygiene Education in Adolescents Based on Psychological Behavioral Theories - A Pilot Study

\author{
V. Brukienë ${ }^{\mathrm{a}}{ }^{*}$, J. Aleksejûnienë ${ }^{\mathrm{b}}$
}

vilma.brukiene@mf.vu.lt

anstitute of Odontology, Faculty of Medicine, Vilnius University, Lithuania; ${ }^{b}$ Department of Oral Health Sciences, Faculty of

Dentistry, The University of British Columbia, Vancouver,

Canada

Aim of the study was to test if psychological behavioral theory based interventions are effective in modifying oral hygiene behavior of adolescents. Material and methods: The sample comprised 348 Lithuanian adolescents aged 12-13 years. Subject groups (school classes) were randomly assigned to either one of three intervention groups (interventions based either on the Precaution Adoption Process Model (PAPM), the Authoritative Parenting Model (APM) or on conventional instruction) or to a control group. Baseline and follow-up individual plaque levels were assessed applying the Quantitative Plaque Percent Index. Changes in mean plaque scores among groups were compared by ANOVA test with Post Hoc Dunnet adjustment. In a three-month follow-up observation mean plaque scores decreased by $23.8 \%(\mathrm{p}<$ $0.001)$ in PAPM group and by $18.3 \%(\mathrm{p}=0.007)$ in APM group. No significant changes were found in the control or in the conventional instruction groups. Interventions based on psychological behavioral theories were more effective in modifying dental health-related behavior of adolescents than conventional instruction.

\section{8 \\ Operative Treatment Performance and Acceptance by Preschoolers with Early Childhood Caries in Bogotá, Colombia

\author{
V. Qvist ${ }^{\mathrm{C}}$ \\ smartignon@gmail.com \\ aUniversidad El Bosque, and bistrict Secretary of Health,
} \\ S. Martignon a , M. Tellez ${ }^{\text {a }, ~ L . M . ~ M a r i n ~}{ }^{\text {a, }}$, J.A. Ruiz a , A. Padilla ${ }^{\text {b }}$ \\ Bogotá, Colombia; 'University of Copenhagen, Denmark}

This study aimed at comparing clinical acceptance, pain intensity and 1-year percentage of failure of three class-II-cavity restorative-techniques: amalgam (A), glass-ionomer (GI), and stainless-steel-crown (SSC). 477 economically deprived 3-5-yearolds in Bogotá, Colombia, who had at least one occlusal-proximal cavitated carious lesion in a primary tooth, participated. Restorations were conducted under cotton-roll isolation as follows: A: local-anesthesia, conventional bur preparation, amalgam placement; GI: no local-anesthesia, bur cavity opening, hand-excavator removal of infected-soft dentine, plastic-matrix, glass-ionomer-placement; SSC: no local-anesthesia; infected-soft dentine hand-excavator removal, conservative preparation, glass-ionomer SSC cementation. During the operative procedures the child's behaviour (Frankl's Behaviour-Rating-Scale) and pain intensity (Reis-Barreto's Visual-Analogue-Scale-of-Faces) were assessed. 
Baseline examination included: df-s and caries-risk-assessment. Mean age at baseline: $3.8 \pm 0.7$ years; random treatment-allocation: A: $168=35 \%$, GI: $162=34 \%$, and SSC: $147=31 \%$. After 12 months, 258 (54\%) restorations were evaluated for failure: dental caries, marginal integrity-loss, restoration/tooth fracture, and pulpal complication. Analyses disclosed no significant differences between remaining and dropout children regarding: age, gender, mean df-s, risk-status, restorative technique, or first-primary-molar-tooth restored (Chi-square test, $p$ values $>0.05$ ). A 'positive' to 'definitely-positive' behaviour and a 'very low' to 'low' pain intensity was observed in over $90 \%$ of the children, with no significant differences between techniques (Chi-square test, $p$ values $>0.05$ ). The lowest failure percentage was observed for SSC (15\%), followed by A (18\%) and GI (53\%), with significant differences between GI and the two other techniques (ANOVA test; $p$ values $<0.05)$. The most prevalent reasons for restoration failure were marginal-integrity alterations, fractures and dental caries. To conclude: There was no difference in children's behavior and pain intensity between the 3 treatments but SSC and A performed significantly better than GI in terms of restoration failure during the first year.

3M-ESPE funded partially this study. The Secretary of District Health - Bogotá contributed with recruiting and following up the sample.

\section{9}

Photodynamic Antimicrobial Chemotherapy in Bacterial Decontamination of Deep Carious Lesions

C.A.B. Guglielmi ${ }^{\text {a }}{ }^{*}$, M.R.L. Simionato ${ }^{\text {b }}$, J.C.P. Imparato ${ }^{\text {a }}$, S.L.Pinheiro ${ }^{C}$, D.P. Raggio ${ }^{a}$, M.A.A.C. Luz ${ }^{\mathrm{d}}$

camigugli@usp.br

aDepartment of Pediatric Dentistry, School of Dentistry,

${ }^{b}$ Department of Microbiology, Institute of Biomedical

Sciences, University of Sao Paulo, 'Department of Pediatric

Dentistry, School of Dentistry, Catholic University of Campinas,

dDepartment of Restorative Dentistry, School of Dentistry,

University of Sao Paulo, Brazil

Background and Objectives: As an alternative to remove cariogenic organisms by drilling, the purpose of this study was to eliminate them through photosensitization promoted by irradiation with a low power laser in combination with a photosensitization dye. Patients and Methods: Remaining dentine from deep carious lesions on permanent molars $(n=26)$ were treated with $0.01 \%$ methylene blue dye and irradiated with a low power laser (InGaAIP - Indium Gallium Aluminum Phosphide; $\lambda=660 \mathrm{~nm}$; $100 \mathrm{~mW} ; 320 \mathrm{Jcm}-2 ; 90 \mathrm{~s}$; 9J). Samples of dentine from pulpal wall were collected with a micropunch immediately before (baseline) and after (experimental) the photodynamic antimicrobial chemotherapy (PACT) and kept in VGMA III transport medium for microbiological analysis. Samples were cultured in Brucella blood agar, Mitis Salivarius Bacitracin agar and Rogosa SL agar plates to determine the total viable bacteria, mutans streptococci and Lactobacillus spp. counts, respectively. After incubation, the number of colony- forming units (CFU) was counted and the percentage of microbial reduction was calculated for each group of bacteria studied. Results: Statistical analyses comparing data of baseline and experimental samples were done trough Wilcoxon test and the comparisons between groups were analyzed though MannWhitney test. PACT leads to a statistically significant reduction $(\mathrm{p}<0.0001)$ of the dentin microflora for mutans streptococci (78.07\%), Lactobacillus spp. (78.0\%) and also for the total of viable bacteria (76.03\%) and that there was no statistic differences for its efficiency between groups ( $p>0.05$ ). Conclusion: Through the methodology tested, PACT can be considered an effective technique for dentin decontamination in deep carious lesions. This study was needed to test the effectiveness of PACT in dentin decontamination in vivo, as a minimally invasive approach, since its potential has been already established in vitro.

This study was supported by FAPESP (Fundacao de Amparo a Pesquisa do Estado de Sao Paulo, Brazil) (\#08/54903-3) and CAPES (Coordenacao de Aperfeicoamento de Pessoal de Nivel Superior, Brazil).

\section{0 \\ Mineral Supplementation of Desalinated Drinking Water for Caries Control

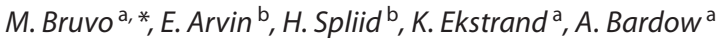 \\ mbl@odont.ku.dk

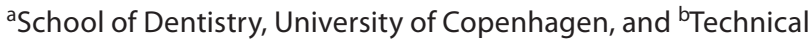 University of Denmark, Denmark}

Shortage of drinking water in many parts of the world will increase the use of desalinated seawater and brackish water as a drinking water resource. After treatment, such water is depleted of ions and therefore some level of remineralisation has to occur. However, apart from the well-documented effects of fluoride little is known about the effect of other ions in drinking water on dental caries. Recently we showed that high levels of calcium in drinking water were related to a low number of decayed, missing and filled tooth surfaces (DMF- S) among 15-year-olds in Denmark. The combined explanatory power for a model containing both fluoride and calcium was $45 \%$. The aim of the present study was to make an expanded model for the effect of all major physicochemical water characteristics on DMF-S. Physiochemical data were retrieved as average concentrations from 1995 to 2004 for each waterworks in Denmark's 275 municipalities from the Geological Survey of Denmark and Greenland (GEUS) and DMF-S data for Danish school children aged 15 in 2004 were retrieved from the Danish National Board of Health. The identification and estimation of the most significant water compounds for DMF-S were performed by an iterative search and testing algorithm based on generalized linear modelling. Among twenty-two different physiochemical variables from drinking water a model containing fluoride (reducing), calcium (reducing), bicarbonate (reducing), $\mathrm{pH}$ (reducing) and chloride (increasing) could be developed increasing the explanatory power from drinking water on DMF-S to $51 \%$. This is the highest explanatory power ever found for simple chemical effects on dental caries in vivo. We therefore conclude that physiochemical characteristics of drinking water other than fluoride and calcium also have an impact on DMF-S. 
Financial support from Institute of Odontology, Faculty of Health Sciences, University of Copenhagen, Danish Dental Association, Danish Medical Research Council and Colgate is gratefully acknowledged.

\section{1}

\section{Root Caries Index as an Outcome Variable in} Regression Modeling of Root Caries

\author{
A.V. Ritter ${ }^{\mathrm{a}, *}$, J.S. Preisser ${ }^{\text {b }}$, B.T. Amaechi ${ }^{\mathrm{C}}$, M.Z. Anabtawi ${ }^{\mathrm{d}}$, \\ S.K. Makhija ${ }^{d}$, D.A. Shugars ${ }^{\text {a }}$, for the X-ACT Collaborative \\ Research Group
}

rittera@dentistry.unc.edu

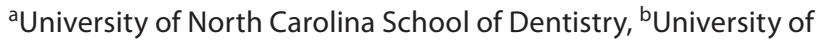
North Carolina Gillings School of Global Public Health, Chapel Hill, N.C., The University of Texas Health Sciences Center at San Antonio, Tex., ${ }^{\mathrm{d}}$ The University of Alabama at Birmingham School of Dentistry, Ala., USA

Person-level prevalence is often used as the outcome variable when estimating associations between risk indicators and root caries (RC). However, prevalence does not account for number of surfaces at risk; therefore prevalence regression models may overlook predictors of disease extent. The root caries index (RCI), defined as the proportion of exposed root surfaces that are affected, may be a more appropriate model outcome. This study used ordinary least squares to assess associations of baseline characteristics with RCI in caries-active adults from an ongoing multi-site clinical trial, X-ACT. Study participants, adults aged 21-80 with at least 12 erupted teeth and between 1-10 coronal or $\mathrm{RC}$ lesions, received caries examinations and responded to demographic and medical-dental questionnaires. Backward selection for multiple linear regression was applied to participants with at least one exposed root surface $(n=558)$ with a $\mathrm{p}$ value criterion of 0.05 or less for inclusion. Heteroscedasticity-consistent standard errors (se) for regression coefficient estimates (est) were used to account for violations of normality and constant variance. RCI was 13.9 ('b 19.6) surfaces per 100 surfaces at risk. Adjusting for study site and participant age, male gender (est = 4.11 , se $=1.62$ ), oral health self-described as poor/very poor, fair or good versus very good/excellent (est $=9.88$, se $=2.90$ ), and fewer teeth (est $=0.67$, se $=0.22$ ) were associated with higher RCI. These results differed slightly from results of a logistic regression analysis for prevalence, in which, adjusting for study site and coronal caries index (coronal surfaces with caries/surfaces at risk), male gender, increased age, fewer teeth, and recent dental visit were significantly associated with higher odds of any RC (personlevel prevalence). The results from the linear modeling of RCI appear to be more consistent with RC etiology than the logistic modeling of prevalence.

Supported by NIDCR grants U01DE018038, U01DE018047, U01DE018048, U01DE018049, and U01DE018050.
62

\section{Caries Status, Salivary Flow and pH in Type 2 Diabetes Mellitus Adults: A Preliminary Study}

\author{
R.A. Giacaman *, D. Ibarra, P. Tapia, V. Mujica \\ giacaman@utalca.cl \\ University of Talca, Chile
}

Aim: Type 2 diabetes mellitus (DM) patients have more prevalence and severity of periodontitis. It is still unclear and controversial whether DM is associated with higher caries risk, nonetheless. While some investigations have shown higher rates of caries among DM individuals, others suggest that DM patients have less caries than non-DM individuals, based on the fact that DM patients have carbohydrate-restricted diets. The aim of this study, therefore, was to assess caries status in DM elderly patients. Materials and Methods: $28 \mathrm{DM}$ individuals and 41 age-matched non-diabetic controls with a mean age of $64.7 \pm 8.6$ years old, participated in this cross-sectional study. All DM patients had been diagnosed at least 5 years before enrolment in the study, and received treatment by a specialist physician. Each subject was interviewed and examined by 2 previously calibrated dentists. Caries status was expressed as DMFT index (WHO). Samples of stimulated saliva to assess salivary flow and $\mathrm{pH}$ were obtained. Comparisons were considered significant at $\mathrm{p}<0.05$ using Student's $\mathrm{t}$ test. Results: $100 \%$ of DM subjects underwent regular control of DM and had received diet instruction and follow up. $92.8 \%$ of the patients had other systemic condition besides DM. DMFT was $14.57 \pm 8.99$ in DM patients and $23.17 \pm 5.3$ in the age-matched controls $(\mathrm{p}<0.001)$. Salivary $\mathrm{pH}$ was significantly higher in $\mathrm{DM}$ subjects than controls $(\mathrm{p}<0.001)$, but stimulated salivary flow failed to show differences between the groups. Conclusions: Within the limitations of this type of study, well controlled DM patients show less history of caries than unaffected controls. Higher salivary $\mathrm{pH}$ in DM patients appears to be associated with lower caries experience in this sample of elderly DM patients under strict control.

Funding: Grant \#I001068 of the Direction of Research Projects (DPI) of the University of Talca. 


\section{3 \\ Use of the Manual of Instruction Program in Education for Health}

S. Groisman ${ }^{\text {a, }}$, J.J. de Soet ${ }^{\text {b }}$, M. Bertolini ${ }^{\text {C }}$, E.R.T. Carvalho ${ }^{\mathrm{c}}$

sonia@dentistas.com.br

aSchool of Dentistry, Federal University of Rio de Janeiro, Brazil;

${ }^{b}$ Department of Oral Microbiology, Academic Center

for Dentistry - ACTA, The Netherlands; 'School of Dentistry,

Estadual University of Rio de Janeiro, Brazil

According to the World Health Organization (WHO) the level of oral health in Brazil is one of the lowest in the world due to lack of information among the population. The objective of this study was to investigate whether dental knowledge promotes a better oral health. 45 students from a public school in Niterói, RJ (age 12 year) participated. They were randomly divided into 3 groups where G1 received health education through a manual of programmed instruction, G2 received conventional lectures on dental hygiene and G3-control received speeches from the $\mathrm{Mu}$ nicipal Dental Health of the City of Niterói. For all students, the Gingival Index (GI), the salivary levels of Streptococcus mutans by using a Strip Mutans test (SSM) and knowledge about etiology and prevention of caries, using a questionnaire was measured before the education and 2 months later. The GI for all three groups reduced significantly with 0.21 for group 1 to 0.36 for group 3 ( $p>0.05$ paired $t$ test). There was no significant difference between the groups. The levels of SSM decreased slightly but a Fisher-exact test revealed that no significant differences could be found between the groups. From the questionnaire, G1 and G2 had 41 questions wrong before the education and 13 after the education, while G3 decreased from 41 to 38 wrong questions. G1 and $\mathrm{G} 2$ reacted significantly better to the education than G3 ( $p<$ 0.05 ) We conclude that the Instruction Manual and the Scheduled Lecture were effective in gaining dental awareness which was visible in de reduced gingival plaque index. Although differences between the studied groups were not significant, we recommend implementation of this program to reduce the dental caries risk in these populations.

Supported by Federal University of Rio de Janeiro.

\section{4 \\ Clinical and Non-Clinical Characteristics of Participants and Non-Participants of a RCT on Caries-Preventive Strategies in the Netherlands}

J.H. Vermaire*, C. van Loveren, J. Hoogstraten

e.vermaire@acta.nl

ACTA, Amsterdam, The Netherlands

Studies reporting clinical and non-clinical outcomes between participant and non-participants are scarce. Results from previous studies show that non-participants are more likely to show less favorable outcomes on socio-economic issues as well as on caries experience. The following situation occurred: As part of baseline data-collection of a RCT on caries-preventive strategies, 346 parents of children of 6.0 years old ( \pm 3 months) were approached to let their child participate. 60 parents refused but 56 parents were willing to fill out the same set of questionnaires and allowed their child to be clinically examined. Comparison revealed that parents from participating children had higher SES, were more often of autochthonous origin and scored better on knowledge questions. Furthermore these parents reported a higher willingness to invest (money and time) to keep their child's teeth healthy. Participating children were more likely to hold on to regular meals and had lower levels of plaque (OHI-s: 0.89 $( \pm 0.71)$ vs $1.25( \pm 0.67) \mathrm{t}=-3.13, \mathrm{p}<0.005)$. Surprisingly, nonparticipating children had lower dmfs scores ( $4.69 \pm 6.88$ vs 5.85 \pm 8.76). Correcting for SES (ANCOVA) would polarize the results even more. The care index ( $\mathrm{fs} / \mathrm{ds}+\mathrm{fs})$ of non-participants was lower $(0.37( \pm 0.36)$ vs $0.58( \pm 0.40), Z=-2.68, p<0.01$. Including extractions in the concept of 'care' ( $\mathrm{ms}+\mathrm{fs} / \mathrm{ds}+\mathrm{ms}+\mathrm{fs})$ resulted in higher levels of care $(0.43( \pm 0.39)$ vs $0.66( \pm 0.40), Z=-3.10, p<$ $0.005)$. Apparently, dietary habits, dental knowledge and oral hygiene are not the only modifying factors involved in the development of caries in children. Other factors may contribute to differences found between participants and non-participants of this RCT. These should be taken in mind when interpreting the outcomes of clinical trials. 


\section{Caries Research}

\section{Session 4 Fluoride}

\section{5}

Effect of $\mathrm{TiF}_{4}, \mathrm{ZrF}_{4}, \mathrm{HfF}_{4}$ and $\mathrm{AmF}$ on Erosion and Erosion/Abrasion of Enamel and Dentin in situ

A. Wiegand ${ }^{\mathrm{a}, *}$, B. Hiestand ${ }^{\mathrm{a}}$, B. Sener ${ }^{\mathrm{a}}$, A.C. Magalhaes ${ }^{\mathrm{b}}$,

M. Roos ${ }^{\mathrm{C}}$, T. Attin ${ }^{\mathrm{a}}$

annette.wiegand@zzmk.uzh.ch

${ }^{a}$ Clinic for Preventive Dentistry, Periodontology and Cariology, University of Zurich, Switzerland; 'b Department of Biological Sciences, Bauru School of Dentistry, Bauru, Brazil; 'Biostatistics Unit, Institute of Social and Preventive Medicine, University of Zurich, Switzerland

This in situ study aimed to analyse the impact of different tetrafluorides $\left(\mathrm{TiF}_{4}, \mathrm{ZrF}_{4}\right.$ and $\mathrm{HfF}_{4}$ ) and $\mathrm{AmF}$ on erosion and erosion plus abrasion of enamel and dentin. Ten volunteers took part in this crossover and double-blind study performed in 8 phases of each 3 days. In each phase, 2 bovine enamel and 2 dentin specimens were fixed in intraoral appliances. One enamel and one dentin sample were pretreated once with $\mathrm{TiF}_{4}, \mathrm{ZrF}_{4}, \mathrm{HfF}_{4}$ or AmF (all $0.5 \mathrm{M} \mathrm{F}$ ) for $60 \mathrm{~s}$, while the other samples remained unfluoridated and served as control. Then, all samples were subjected to either erosion only (4 times/day, $90 \mathrm{~s}$, extraoral immersion in Sprite) or to erosion and abrasion (2 times/day, 30s/sample). Toothbrushing abrasion was performed extraorally $90 \mathrm{~min}$ after the first and last erosion with an electrical toothbrush and fluoridated toothpaste at $1.2 \mathrm{~N}$. After 3 days, enamel and dentin loss was assessed by profilometry $(\mu \mathrm{m})$ and analysed by repeated measures ANOVA and paired t-test $(p<0.05)$. All fluoride solutions reduced enamel and dentin loss significantly compared to the controls. Generally, eroded samples showed less wear than eroded and abraded samples. The protective potential of the fluorides was not significantly different and was only slightly, but mostly not significantly, decreased by abrasion. The protective effect of the fluoride solutions was similar in enamel and dentin. Tetrafluorides and AmF are able to reduce erosion and erosion plus abrasion in situ and are almost equally effective.

\author{
66 \\ Protective Effect of $\mathrm{TiF}_{4}$ Solution with Different \\ Concentrations and pH on the Development of \\ Erosion-Like Lesions \\ L.H. Hove ${ }^{\mathrm{a}, *}$, B. Holme ${ }^{\mathrm{b}}$, K.R. Stenhagen ${ }^{\mathrm{a}}$, A.B. Tveit $^{\mathrm{a}}$ \\ lenehh@odont.uio.no \\ aniversity of Oslo, and ${ }^{\mathrm{b}}$ SINTEF, Oslo, Norway
}

The erosion-inhibiting effect of various TiF4 solutions has been investigated in many in vitro studies with different results. The aim was to study how concentration and $\mathrm{pH}$ of TiF4 solutions influence the protective effect on enamel when exposed to acid. Materials and method: Eight teeth were all divided into five specimens. One specimen from each tooth served as a control. The remaining four were treated separately with either 1 . $\operatorname{TiF}_{4}(0.5 \mathrm{M}$ F), 2. $\mathrm{TiF}_{4}(0.05 \mathrm{M} \mathrm{F}), 3 . \mathrm{TiF}_{4}(0.5 \mathrm{M} \mathrm{F}$ buffered to the same $\mathrm{pH}$ as $0.05 \mathrm{M} \mathrm{F})$ or $4 . \mathrm{TiF}_{4}(0.05 \mathrm{M} \mathrm{F}$ buffered to the same $\mathrm{pH}$ as $0.5 \mathrm{M} \mathrm{F})$ for $2 \mathrm{~min}$ prior to exposure to $0.01 \mathrm{M} \mathrm{HCl}$ for $2+2+2 \mathrm{~min}$ and analyzed by a White Light Interferometer. A coating was present on the enamel in all groups, except in group 4, after 2 min of acid exposure. The fraction of the area covered with the coating was $93,71,17$ and $0 \%$ in group $1-4$. When present, the coating protected the surface completely from the acid. After 6 min of acid exposure, remnants of the coating could only be seen in group 1 , where $43 \%$ of the surface was covered. In all specimens, the uncovered enamel was etched as the control $(1.2 \mu \mathrm{m} / \mathrm{min})$ except for the specimens in group $4(1.7 \mu \mathrm{m} / \mathrm{min})$. In group 1 , the mean etch depth was reduced by $47 \%$ compared to the controls after $4 \mathrm{~min}$ exposure to acid. No significant reduction was shown for the other groups. Reducing the concentration of $\mathrm{TiF}_{4}$ and increasing the $\mathrm{pH}$ of the solution decreased the ability of native $\mathrm{TiF}_{4}(0.5 \mathrm{M} \mathrm{F})$ to protect enamel from acid attacks.

Funding: University of Oslo, Norway. 


\section{7 \\ Effect of $\mathrm{TiF}_{4}$ and NaF Varnishes and Solutions on Enamel Erosion plus Abrasion in vitro}

L.P. Comar * F.M. Levy, M.F. Gomes, D. Rios, M.A.R. Buzalaf,

A.C. Magalhães

acm@usp.br

Bauru School of Dentistry, University of São Paulo,

Bauru, Brazil

Previous in vitro study has shown that TiF4 varnish might reduce enamel erosion [Magalhães et al.: Caries Res. 2008;42:269274]. However, no data regarding the effect of this experimental varnish on enamel erosion plus abrasion is available so far. Thus, this in vitro study aimed to analyze the effect of a single application of TiF4 and $\mathrm{NaF}$ varnishes and solutions to protect against enamel erosion plus abrasion. Bovine enamel specimens were pretreated with NaF-Duraphat varnish (2.26\% F, pH 4.5), $\mathrm{NaF} \mathrm{CaF}_{2}$ Duofluorid varnish (5.63\% F, pH 8.0), NaF- experimental varnish (2.45\% F, pH 4.5), $\mathrm{TiF}_{4}$-experimental varnish (2.45\% F, pH 1.2), $\mathrm{NaF}$ solution $(2.26 \% \mathrm{~F}, \mathrm{pH} 4.5), \mathrm{TiF}_{4}$ solution $(2.45 \% \mathrm{~F}, \mathrm{pH} 1.2)$ and placebo varnish ( $\mathrm{pH} 5.0$, no-F varnish control). Controls remained untreated. Ten specimens in each group were then subjected to an erosive demineralization (Sprite Zero) for $90 \mathrm{~s}, 4$ times daily. Additionally, the specimens were abraded using an electrical toothbrush (Colgate Montions Multi-action, F = 1.5 N) and slurries of no-F toothpaste (1:3 water, $0.5 \mathrm{ml} / \mathrm{specimen}$, Crest Procter \& Gamble, $\mathrm{pH} 6.8$, silica) for $10 \mathrm{~s}, 2$ times daily, after the first and last erosive challenges. Between the erosive and abrasive challenges, the specimens were remineralized using artificial saliva ( $\mathrm{pH}$ 6.8). Enamel loss was measured profilometrically $(\mu \mathrm{m})$ after the 5 th day. The data were analyzed by ANOVA followed by Tukey's test $(p<0.0001)$. Erosion was significantly lower for $\mathrm{TiF}_{4}$ varnish-treated specimens compared to the specimens treated with the NaF-based varnishes and solution, which in turn showed significantly less erosion than control and placebo varnish. $\mathrm{TiF}_{4}$ solution lead to the highest erosive loss compared to all groups. In conclusions, $\mathrm{TiF}_{4}$ varnish seems to be a promising treatment to reduce enamel loss under mild erosive and abrasive conditions.

Funding: FAPESP - São Paulo State Research Foundation (Process no. 08/07105-4 and 08/03727-0).

\section{8 \\ Effect of $\mathrm{TiF}_{4}$ and NaF Varnishes and Solutions on Dentin Erosion Plus Abrasion in vitro}

F.M. Levy*, F.A.P. Rizzante, D. Rios, A.C. Magalhães, M.A.R. Buzalaf mbuzalaf@fob.usp.br

Bauru School of Dentistry - University of São Paulo, Brazil

Previous in vitro study has shown that $\mathrm{TiF}_{4}$ varnish might reduce enamel erosion [Magalhães et al.: Caries Res. 2008;42:269274] but not dentin erosion [Magalhães et al.: J Dent. 2009 Oct 4 (Epub ahead of print)]. However, no data regarding the effect of this experimental varnish on dentin erosion plus abrasion is available so far. Thus, this in vitro study aimed to analyze the effect of a single application of $\mathrm{TiF}_{4}$ and $\mathrm{NaF}$ varnishes and solutions to protect against dentin erosion plus abrasion. Bovine dentin specimens were pre-treated with NaF-Duraphat varnish $(2.26 \% \mathrm{~F}, \mathrm{pH}$ 4.5), NaF/CaF 2 -Duofluorid varnish (5.63\% F, pH 8.0), NaF-experimental varnish $(2.45 \% \mathrm{~F}, \mathrm{pH} 4.5), \mathrm{TiF}_{4}$-experimental varnish (2.45\% F, pH 1.2), NaF solution (2.26\% F, pH 4.5), $\mathrm{TiF}_{4}$ solution (2.45\% F, pH 1.2) and placebo varnish ( $\mathrm{pH} 5.0$, no-F varnish control). Controls remained untreated. The varnishes were applied in a thin layer and removed after $6 \mathrm{~h}$ and the solutions were applied for $1 \mathrm{~min}$. Ten specimens in each group were then subjected to an erosive demineralization (Sprite Zero) for 90 s, 4 times daily. Additionally, the specimens were abraded using an electrical toothbrush (Colgate Montions Multi-action, $\mathrm{F}=1.5 \mathrm{~N}$ ) and slurries of no-F toothpaste (1:3 water, $0.5 \mathrm{ml} /$ specimen, Crest - Procter \& Gamble, pH 6.8, silica) for $10 \mathrm{~s}, 2$ times daily, after the first and last erosive challenges. Between the erosive and abrasive challenges, the specimens were remineralized using artificial saliva $(\mathrm{pH}$ 6.8). Enamel loss was measured profilometrically $(\mu \mathrm{m})$ after 5 th day. The data were analyzed by ANOVA followed by Tukey's test $(\mathrm{p}<0.05)$. All fluoridated varnishes were able to significantly reduce wear when compared to placebo varnish, control and fluoridated solutions, which did not significantly differ from each other. In conclusion, all fluoridated varnishes tested had a similar performance and seem to have a good potential to reduce dentin loss under mild erosive and abrasive conditions.

Supported by São Paulo State Research Foundation- FAPESP (Proc. 08/07105-4 and 08/03727-0).

\section{9 \\ Dental Erosion Inhibition by $\mathrm{SnF}_{2}$ and $\mathrm{TiF}_{4}$ Compared with the Effect of HF}

A. Young ${ }^{\mathrm{a}, *}$, G. Jonski ${ }^{\mathrm{b}}$, G. Rölla ${ }^{\mathrm{b}}$

a.y.vik@odont.uio.no

aDepartment of Cariology and Gerodontology, Institute of

Clinical Dentistry, and ${ }^{\mathrm{b}} \mathrm{Clinical}$ Research Laboratory, Faculty of

Dentistry, University of Oslo, Norway

Background: The aim of this in vitro pilot study was to compare the effect of three acidic equimolar fluoride solutions (and subsequent water treatment to remove all un-reacted fluoride) on the acid resistance of teeth. Cleaned whole teeth $(n=24,6$ teeth/ group) were each pre-etched by immersion in $10 \mathrm{ml}$-aliquots of $0.1 \mathrm{~mol} / \mathrm{l}$ citric acid for $5 \mathrm{~min}$. The teeth were then incubated in $10 \mathrm{ml}$ of the test fluoride solutions containing $0.1 \mathrm{M} \mathrm{F}: \mathrm{SnF}_{2}(\mathrm{pH}$ 2.9), $\mathrm{TiF}_{4}(\mathrm{pH}$ 2.0) or hydrofluoric acid (HF, pH 3.0) for $20 \mathrm{~min}$. A control group was not treated with any fluoride. Teeth were then incubated in distilled water for $24 \mathrm{~h}$ before re-etching as for the pre-etch. The citric acid solutions before and after F-treatment were retained for $\mathrm{Ca}$-analysis using atom absorption spectroscopy. Mean pre- and post etch [Ca] for each F-solution and $\% \Delta \mathrm{Ca}$ were calculated for each F-solution and for the control. Mean preetch [Ca] and mean post F-treatment [Ca] with $(\mathrm{SD})$ results in $\mathrm{mg} / \mathrm{l}$ were for HF: 8.894 (2.52) and 1.418 (1.188); $\mathrm{SnF}_{2}: 8.201$ (1.92) and 2.525 (1.19); $\mathrm{TiF}_{4}: 7.683$ (1.75) and 2.822 (0.93) and control: 7.318 (2.31) and 7.470 (1.69). The \% reduction in Ca dissolution was $86,70,64$ and -6 , for $\mathrm{HF}, \mathrm{SnF}_{2}, \mathrm{TiF}_{4}$ and the control respec- 
tively. At this low fluoride concentration, HF was more effective than the two metal fluorides, suggesting that the metal ions may not contribute directly to the inhibition of mineral dissolution. The role of the metal ions may be to provide an acidic environment in these solutions (and formation of HF), as they react with the hydroxyl ions of water during the solubilization of their salts.

Supported by Research grant from the University of Oslo.

\section{0}

\section{Erosion-Inhibiting Potential of a Stannous Chloride-Containing Fluoride Solution under Acid Flow Conditions in vitro}

H. Yu ${ }^{\text {a, b, }}{ }^{*}$, F.J. Wegehaupt ${ }^{\text {b }}$, M. Zaruba ${ }^{\text {b }}$, M. Roos ${ }^{c}$, T. Attin $^{\text {b }}$, A. Wiegand ${ }^{\mathrm{b}}$

hao.yu@zzmk.uzh.ch

aDepartment of Prosthodontics, Wuhan University, China;

${ }^{b}$ Clinic for Preventive Dentistry, Periodontology and Cariology,

University of Zurich, 'Biostatistics Unit, Institute of Social and

Preventive Medicine, University of Zurich, Switzerland

This study aimed to analyse the erosion-inhibiting potential of a single application of stannous chloride-containing fluoride solution on pellicle-covered enamel and dentin under constant acid flow conditions in vitro. Bovine enamel $(n=60)$ and dentin $(n=60)$ samples were exposed to the oral cavity of four healthy volunteers to allow for in situ pellicle formation. Pellicle-covered samples were randomly assigned to three groups (each $n=20$ enamel and $\mathrm{n}=20$ dentin samples; 5 enamel and 5 dentin samples/volunteer) and treated once with a $\mathrm{SnCl}_{2} / \mathrm{AmF} / \mathrm{NaF}(800$ ppm Sn(II), 500 ppm F, pH 4.5) or a NaF solution (500 ppm F, pH $4.5)$ for 2 min or remained untreated (controls). Samples were eroded with hydrochloric acid ( $\mathrm{pH} 2.6)$ in a small erosion chamber at $60 \mu \mathrm{l} / \mathrm{min}$ for $25 \mathrm{~min}$. Calcium release into the acid was monitored in consecutive $30 \mathrm{~s}$ intervals for $5 \mathrm{~min}$, then at $1 \mathrm{~min}$ intervals up to a total erosion time of 25 min using the Arsenazo III procedure [Attin et al.: Caries Res 2005;39:432-6]. Data were statistically analysed by random-effects linear models $(\mathrm{p}<0.05)$. The stannous chloride-containing fluoride solution reduced calcium loss of enamel and dentin to up to $6 \mathrm{~min}$ and $3.5 \mathrm{~min}$, respectively. Calcium loss (\% of control) amounted from $24 \pm 7$ (30 s) up to $93 \pm 14(6 \mathrm{~min})$ in enamel and from $38 \pm 13(30 \mathrm{~s})$ to $87 \pm 15(3.5 \mathrm{~min})$ in dentin. The sodium fluoride solution was unable to reduce enamel and dentin erosion at any timepoint. In conclusion, a single application of a stannous chloride-containing fluoride solution reduced enamel and dentin erosion up to $6 \mathrm{~min}$ and 3.5 min of constant acid flow, respectively.

The study was supported by GABA International AG, Switzerland.

\section{1 \\ Superior Protection of Stabilized, Stannous-Containing Toothpaste against Erosive Acid Damage}

\author{
R.V.Faller*, S.L. Eversole \\ faller.r@pg.com \\ Procter \& Gamble Co., Mason Ohio, USA
}

Toothpaste benefits can be measured not only by prevention against subsurface damage but also by assessing a product's ability to protect enamel against irreversible erosive acid damage. We previously demonstrated the ability of $\mathrm{SnF}_{2}$ toothpaste to provide superior protection against erosive acid challenges in both in vitro and in situ studies. Objectives: Purpose of this study was to compare the ability of a new, stabilized stannous-containing $\mathrm{NaF}$ toothpaste (Crest Pro-Expert Enamel protection) to protect enamel against erosive acid damage relative to a number of marketed toothpastes. This next-generation toothpaste features $\mathrm{NaF}$ $(1,450 \mathrm{ppm} \mathrm{F}-)$ as the active ingredient and stannous chloride as a key excipient. These elements combine synergistically during toothbrushing to generate a stannous-fluoride complex. Methods: Cores of human enamel (4/group) were soaked in pooled human saliva (pellicle formation), and treated with a 1:3 slurry (toothpaste:saliva) using a standardized model (5-day cycling) that includes $1 \%$ citric acid (10 min) challenges $60 \mathrm{~min}$ after each treatment. Enamel surface loss was measured using TMR. Results: Specimens treated with the new, stabilized Sn-containing formula resulted in the least damage, with Crest Pro-Expert toothpaste providing significantly greater protection $(\mathrm{p}<0.05$, Fisher LSD) compared to all other products tested. Other toothpastes tested resulted in 1.8 to 3.4 times more damage compared to this new Sn-containing toothpaste. Other products: active agent(s): enamel loss data include: Meridol: 1,400 ppm $\mathrm{F}$ as $\mathrm{AmF}+\mathrm{SnF}_{2}: 12.0 \mu \mathrm{m}$; Colgate Cavity Protection: $1,450 \mathrm{ppm} \mathrm{F}$ as SMFP + NaF: $12.9 \mu \mathrm{m}$; Odol-med 3: 1,400 ppm F as NaF: $14.2 \mu \mathrm{m}$; Elmex: 1,400 ppm F as AmF: $14.5 \mu \mathrm{m}$; Colgate Enamel Protect: 1,450 ppm $\mathrm{F}$ as $\mathrm{NaF}+\mathrm{KNO}_{3}: 16.3 \mu \mathrm{m}$; Lacalut: $1,400 \mathrm{ppm} \mathrm{F}$ as $\mathrm{AlF}_{3}$ : $18.5 \mu \mathrm{m}$; Sensodyne ProNamel: $1,450 \mathrm{ppm} \mathrm{F}$ as $\mathrm{NaF}+$ $\mathrm{KNO}_{3}: 20.5 \mu \mathrm{m}$; and Mentadent: 1,450 ppm F as NaF + ZnCitrate: $22.3 \mu \mathrm{m}$. Conclusions: These results continue to support the superior protective nature of stabilized, stannous-containing toothpaste against erosive acid damage.

Funding: Procter \& Gamble Company.

\section{2 \\ Reduction of Erosive Wear in situ by Stannous Fluoride-Containing Toothpaste \\ M.C.D.N.J.M. Huysmans *, J.L. Ruben, C. Klijn, D. Unk \\ m.c.d.n.j.m.huysmans@dent.umcn.nl \\ Department of Dentistry, Radboud University Nijmegen \\ Medical Centre, The Netherlands}

The aim of this study was to evaluate the effect of toothpastes with stannous fluoride in the prevention of erosive enamel wear. A combined split-mouth (extra-oral water or toothpaste brushing) and cross over (type of toothpaste) in situ set-up was used. Twelve 
volunteers wore palatal appliances containing 2 control and 2 experimental human enamel samples for experimental runs of 5 days during working hours. Three toothpastes were used, in consecutive runs, in randomized order: two toothpastes containing stannous fluoride (coded $\mathrm{M}$ and $\mathrm{PE}$ ) and one toothpaste containing only sodium fluoride (coded C). On day 1 of each run the appliances were worn for pellicle formation, and not brushed or eroded. At the start and end of days 2 to 5 the samples were brushed using a brushing machine (10 strokes) with a toothpaste- water slurry (ratio $1 / 3 \mathrm{w} / \mathrm{w}$ ) or only water (control) and remained in the slurry or water for $2 \mathrm{~min}$. Erosion took place on days 2 to 5 extra-orally 3 times a day ( $5 \mathrm{~min}$ ) in a $0.05 \mathrm{M}$ citric acid solution $(\mathrm{pH} 2.3)$. Enamel wear depth was quantified by optical profilometry at day 5 . The effect of toothpastes and differences between toothpastes were tested using paired T-test $(\alpha=0.05)$. Average erosive wear depth of control samples in the three runs was 22.3, 23.4, and 24.7 $\mu \mathrm{m}$, respectively, showing a small but significant run effect. Both stannous fluoride toothpastes significantly reduced erosive wear: M by 34\% (SD 39\%) and PE by 26\% (SD 25\%). The control toothpaste reduced erosive wear by $7 \%$ (SD 20\%), but this was not statistically significant. We conclude that stannous fluoride containing toothpaste may help reduce erosive tooth wear.

\section{3}

\section{Effects of Amine Fluoride/Stannous Fluoride} Products on Oral Microflora in Orthodontic Patients

G. Nagy ${ }^{a, *}$, J. Bánóczy ${ }^{b}$, G. Götz $^{c}$, B. Szádeczky ${ }^{c}$, S. Márton $^{\text {d, }}$ M. Madléna ${ }^{c}$

\section{madlena@fok.usn.hu}

${ }^{a}$ Departments of Oral Diagnostics, ${ }^{b}$ Oral Biology Faculty of Dentistry, Semmelweis University, 'Department of Pediatric Dentistry and Orthodontics, Faculty of Dentistry, Semmelweis University, Budapest, ${ }^{d}$ Department of Sociology and Social

Policy, Faculty of Arts, University of Debrecen, Hungary

The aim of the study was to evaluate the effects of amine fluoride/stannous fluoride $\left(\mathrm{AmF} / \mathrm{SnF}_{2}\right)$ containing (meridol) toothpaste and mouthrinse on oral microflore in patients having fixed orthodontic appliances. 40 volunteers (mean age: $20.1 \pm 5.65$ years) participated in the study. Test Group: Participants using meridol toothbrush, toothpaste for twice daily toothbrushing for three minutes, after rinsing with $10 \mathrm{ml}$ mouthrinse for $30 \mathrm{~s}$. Control group: participants using only toothbrush and toothpaste for twice daily toothbrushing for three minutes. Changes in accounts of SM, $\mathrm{LB}$ and six periopathogen species were determined from the plaque samples. Both LB and SM counts were lower after the usage of meridol products. The improvement was slightly better in test group. The LB count values were reduced significantly by 84.6 and $96 \%$ in control and test groups, respectively. The SM count showed a similar tendency ( 59.4 and $77.5 \%, \mathrm{p}<0.01$, respectively). In the test group (using meridol toothpaste and mouthrinse) the level of all pathogenic bacteria showed a very impressive decrease, the relative proportion of periopathogens to total bacterial load was decreased about to the third in this group. In conclusion: The additional use of $\mathrm{AmF} / \mathrm{SnF}_{2}$ containing mouthrinse resulted in a clinically important improvement of gingival health in orthodontic patients.

Supported by GABA International, Therwil, Switzerland.

\section{4 \\ Combination of APF Application and F-Dentifrice Use on Root Dentine Demineralization and Biofilm Composition}

C.P.M. Tabchoury ${ }^{a}$ * , G.C. Vale a, A.A. Del Bel Cury a , L.M.A. Tenuta ${ }^{a}$, J.M. ten Cate ${ }^{\mathrm{b}}$, J.A. Cury ${ }^{\mathrm{a}}$

cinthia@fop.unicamp.br

apiracicaba Dental School, UNICAMP, Piracicaba, Brazil;

${ }^{b}$ Academic Centre for Dentistry Amsterdam, The Netherlands

The objective of this study was to evaluate the effect of acidulated phosphate fluoride (APF) application and fluoridated dentifrice (FD) use and their combination on root caries, biofilm composition and $\mathrm{F}$ reactivity with dentine. A crossover blind in situ study was conducted, in which 12 adult volunteers wore palatal appliances, containing bovine dentine slabs of known surface hardness (SH), which were subjected during 4 experimental phases of 7 days each to the following treatments: placebo dentifrice (PD), 1,100 ppm FD, APF+PD and APF+FD and to a high cariogenic challenge (biofilm accumulation and sucrose solution exposure $8 \times /$ day). APF application was performed at the beginning of the phase once with APF gel $(1.23 \%$ F) for 4 min. Dentifrices were used $3 \times /$ day. At the end of each phase, the plaque-like biofilm (PLB) was collected for F analysis in the fluid. In dentine, mineral loss was quantified by the percentage of SH loss (\%SHL) and transversal microradiography and $\mathrm{CaF} 2$ in dentine was determined. ANOVA showed statistically significant effect $(\mathrm{p}<$ 0.05 ) for the isolated factors dentifrice and APF, but not for the interaction between them ( $p>0.05)$, for \%SHL, integrated mineral loss (IML), F in PLB fluid and CaF2. The results (mean \pm SD) for the groups NFD, FD, APF+NFD and APF+FD were respectively: \%SHL: $71.2 \pm 7.3,38.0 \pm 7.8,52.5 \pm 9.4,24.2 \pm 10.2$; IML $(\operatorname{vol} \% \times \mu \mathrm{m}): 1,951.8 \pm 1,139.6,376.7 \pm 189.4,751.6 \pm 369.2$, $183.9 \pm 41.7 ; \mathrm{F}(\mu \mathrm{M})$ in PLB fluid: $2.2 \pm 1.1,5.4 \pm 3.9,7.5 \pm 6.9$, $8.3 \pm 9.4 ; \mathrm{CaF} 2\left(\mu \mathrm{g} \mathrm{F} / \mathrm{cm}^{2}\right): 2.1 \pm 1.4,6.8 \pm 2.8,21.0 \pm 4.3,24.0$ \pm 5.1 . The data suggest that the combination of APF gel application and the daily use of $\mathrm{F}$ dentifrice is able to reduce root dentine demineralization with synergistic protective effect and is relevant to control root caries.

Supported by FAPESP (process no. 05/03090-4 and 08/116968) and CAPES.

\section{5}

A Calcium Rinse Used before a NaF Rinse Increases Calcium Fluoride in Plaque

G.L. Vogel ${ }^{\mathrm{a}, *}$, L.M.A. Tenuta ${ }^{\mathrm{b}}$, G.E. Schumacher ${ }^{\mathrm{a}}$, L.C. Chow ${ }^{\mathrm{a}}$

jvogel@nist.gov

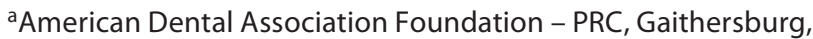
Md., USA; bPiracicaba Dental School, Brazil

'Calcium-fluoride-like' ( $\mathrm{CaF}_{2}$-like) plaque F reservoirs are often identified as a persistent source of plaque fluid F. Previously we have shown (Vogel et al., Cares Res in press) that these deposits were not found in plaque shortly after a $\mathrm{NaF}$ rinse. Objectives: To determine if a Ca pre-rinse can induce the formation $\mathrm{CaF}_{2}$-like deposits in plaque. Methods: Thirteen subjects accumulated 
plaque $48 \mathrm{~h}$ and fasted overnight. The subjects then rinsed with $150 \mathrm{mmol} / \mathrm{l}$ calcium lactate followed immediately by $12 \mathrm{mmol} / \mathrm{l}$ $\mathrm{NaF}$ rinse (both rinses $20 \mathrm{ml}, 1 \mathrm{~min}$ ). One h later a large plaque sample was collected and immediately homogenized under a water-saturated $5 \% \mathrm{CO}_{2}$ atmosphere. The plaque fluid was then recovered and analyzed with microelectrodes. The remaining plaque mass was divided into aliquots. Aliquot 1 was retained, while aliquot 2 was extracted with a fluid having the same $\mathrm{pH}$, $\mathrm{Ca}^{+2}$ activity and $\mathrm{F}$ activity as the plaque fluid (a procedure that removes only $\mathrm{CaF}_{2}$-like deposits). The total $\mathrm{F}$ remaining in the samples was then determined by acid extraction. Results: The plaque fluid $\mathrm{F}$ and total plaque $\mathrm{F}$ were $165 \pm 28 \mu \mathrm{mol} / \mathrm{l}$ and 27.0 $\pm 2.7 \mu \mathrm{mol} / \mathrm{g}$, respectively (mean $\pm \mathrm{SE}$ ). These values are similar to previous values $(\approx 6 \times$ and $10 \times$ higher than values obtained without the pre-rinse [Vogel et al., J Dent Res 2008;87:466-469]). The amount of $\mathrm{CaF}_{2}$-like deposits as determined by subtracting the total $\mathrm{F}$ in aliquot 2 from aliquot 1 , was $9.3 \pm 1.9 \mu \mathrm{mol} / \mathrm{g}$. Conclusion: The Ca pre-rinse induces the formation of large amounts of plaque $\mathrm{F}$, about $1 / 3$ of which appears to be in the form of $\mathrm{CaF}_{2}$ like deposits. These results suggest that a Ca pre-rinse can increase the cariostatic effect of $\mathrm{F}$ topical agents.

Supported by NIDCR grant DE16416 ADAF, and NIST.

\section{6 \\ Low Fluoride Dentifrice and Deciduous Enamel Demineralization under Biofilm Accumulation and Sucrose Exposure}

\section{A.A. Del Bel Cury *, R.C. Amaral, L.M.A. Tenuta, C.P.M. Tabchoury, J.A. Cury \\ altaircury@dglnet.com.br \\ Piracicaba Dental School, UNICAMP, Piracicaba, SP, Brazil}

Since low fluoride (F) dentifrice (500 ppm F) compared with 1,100 ppm $\mathrm{F}$ is not effective to control caries in caries active children, this experimental in situ study was conducted to test the hypothesis whether its effect would depend on the current cariogenic challenge. During four phases of 14 days each, 14 volunteers used 500 or 1,100 ppm F dentifrice, and wore palatal appliances containing deciduous enamel slabs, which were subjected to increasing cariogenic challenge induced by sucrose $20 \%$ exposure from 2 to 8 times/day, and under plaque-like biofilm (PLB) accumulation. On the 14th day, PLB formed on the slabs was collected before ( $10 \mathrm{~h}$ after the last use of dentifrice) and $15 \mathrm{~min}$ after toothbrushing for F determination. Enamel demineralization was assessed by surface hardness loss (\%SHL) and cross-sectional hardness (deltaS = area of hardness loss). F uptake by enamel was also determined. F in PLB fluid and solids was statistically higher when 1,100 ppm F dentifrice was used $(\mathrm{p}<0.05)$. The dentifrices did not differ statistically in terms of \%SHL or deltaS ( $p>0.05)$ but the relationship between deltaS and frequency of sucrose exposure was statistically significant only for the $500 \mathrm{ppm} F$ dentifrice $(p<0.05)$. The dentifrices did not differ statistically in terms of $\mathrm{F}$ in enamel $(\mathrm{p}>0.05)$. The findings suggest that low $\mathrm{F}$ dentifrice may have the same anticaries benefit than 1,100 ppm F dentifrice when the cariogenic challenge provoked by sucrose is not so high.

Supported by FAPESP 2007/05994-3.

\section{7 \\ Evaluation of Dentifrice with Low Fluoride Content Supplemented with Trimetaphosphate on Biofilm and Enamel Demineralization in an in situ Study}

E.M. Takeshita *, L.P. Castro, M. Danelon, K.T. Sassaki, A.C.B. Delbem

elimitsue@yahoo.com.br

UNESP - Sao Paulo State University, Aracatuba, Brazil

The reduction of fluoride $(\mathrm{F})$ concentration in dentifrices would contribute to reduce the prevalence of dental fluorosis. However, it is necessary to maintain their effectiveness. The aim of this study was to evaluate whether the supplementation with sodium trimetaphosphate (TMP) of a dentifrice with low-F content $(500 \mu \mathrm{g} / \mathrm{g})$ would provide a similar effect to that of a standard dentifrice $(1,100 \mu \mathrm{g} \mathrm{F} / \mathrm{g})$. In this crossover double blind study, 9 volunteers, wearing acrylic palatal appliance containing 4 enamel bovine teeth, were subjected to 4 treatment groups: placebo (negative control), dentifrice with low-F, dentifrice with low-F and $1 \%$ of TMP and a dentifrice with $1,100 \mu \mathrm{g} F / \mathrm{g}$ (positive control). During the experimental period (14 days each), test dentifrices were applied $2 \times /$ day, and a $20 \%$ sucrose solution was applied $6 \times /$ day by being dripped on the blocks. After each phase, cross-sectional hardness was assessed to calculate the integrated loss of subsurface hardness $(\Delta \mathrm{KHN})$ and fluoride $(\mathrm{F})$, calcium (Ca), phosphorus $(\mathrm{P})$ and insoluble polysaccharide extracellular (IP) present on biofilm were also measured. The data were heterogenic and statistical analysis was carried through KruskalWallis. The results showed that the dentifrice supplemented with $\mathrm{F}$ and $1 \%$ TMP showed the lowest $\Delta \mathrm{KHN}(\mathrm{p}<0.05)$. Regarding F, $\mathrm{Ca}$ and IP on biofilm there were no differences between dentifrice with low-F and TMP and positive control ( $p>0.05$ ), but they were different when compared to dentifrice with low $\mathrm{F}$ and placebo $(p<0.05)$. It is concluded that the addition of $1 \%$ of TMP to the $500 \mu \mathrm{g} \mathrm{F} / \mathrm{g}$ dentifrice allowed a similar effect as compared with a standard dentifrice in this in situ model.

Supported by FAPESP (Grant no. 07/03254-2, 07/03660-0).

\section{8 \\ Factors Influencing Fluoride Ingestion from Dentifrice by Children}

\section{J.P. Pessan *, C.A.N. Kobayashi, F.M. Italiani, M.R. Belini, L.T. Grizzo, M.A.R. Buzalaf}

mbuzalaf@fob.usp.br

Bauru Dental School, University of São Paulo, Bauru, Brazil

The aim of this study was to assess fluoride ingestion from dentifrice by children, taking into account the amount of dentifrice used during toothbrushing and the dentifrice flavour. Sample consisted of 155 children of both genders attending public kindergartens and schools in Bauru, Brazil, distributed into 5 groups ( $\mathrm{n}=30-32$ ) of children aged 2, 3, 4, 5 and 6 years old. The dentifrices used were Sorriso ${ }^{\mathrm{TM}}$ (1,219 ppm F, pepper-mint flavoured) and Tandy ${ }^{\mathrm{TM}}$ (959 ppm F, tutti-frutti flavoured). The assessment of fluoride intake from dentifrices was done 6 times for each child, by using 0,3,0,6 and 1,2 $\mathrm{g}$ of each dentifrice, following a random, 
crossover distribution. Brushing was performed by the children with or without the assistance of the parents and under the observation of the examiner. Depending on the home habits, children expectorated and rinsed the mouth after brushing or not. The expectorant was collected in a plastic vessel; the toothbrush was rinsed in deionized water, and the rinse was collected in the same vial. Fluoride was analyzed with an ion-specific electrode after HMDS-facilitated diffusion. The amount of fluoride ingested was indirectly obtained. Results were analyzed by 3 -way repeatedmeasures ANOVA and Tukey's test $(\mathrm{p}<0.05)$. An inverse relationship was found between age and fluoride intake for both dentifrices and the 3 amounts used $(p<0.0001)$. Fluoride intake was significantly higher after the use of Tandy ${ }^{\mathrm{TM}}$ under all conditions of the study when compared to Sorriso ${ }^{\mathrm{TM}}(\mathrm{p}<0.0001)$. Significant differences were observed when brushing with $0.3 \mathrm{~g}$ when compared to $1.2 \mathrm{~g}$, for both dentifrices tested $(\mathrm{p}<0.05)$. The results indicate that these variables must be considered in preventive measures aiming to reduce the amount of fluoride ingested by young children.

This study was funded by FAPESP (Grant 2008/09059-0).

\section{9 \\ Urinary Fluoride Excretion in Children Aged 4-6 Years Residing in a $\mathbf{F}$ Area in the UK}

N. Omid ${ }^{\mathrm{a}, *}$, A. Batterham ${ }^{\text {a }}$, W.T. O'Hare ${ }^{\mathrm{a}}$, A. Maguire ${ }^{\mathrm{b}}$, V. Zohoori $^{\mathrm{a}}$

g7128303@tees.ac.uk

aTeesside University, Middlesbrough, ${ }^{\mathrm{b}}$ Newcastle University,

Newcastle Upon Tyne, UK

Background: A range of urinary fluoride excretions (UFE) for children and adults has been provisionally recommended by the WHO [Marthaler: World Health Organisation 1999;70] as a marker for optimal use of fluoride $(F)$ to achieve effective dental caries prevention as well as predict occurrence of dental fluorosis. However, the rate of UFE may vary between days depending on the amount of $\mathrm{F}$ ingested. Therefore, the aims of this study were to investigate daily variation in UFE of 4-6 year-old children residing in a fluoridated area, and compare the range of UFEs with the provisional WHO standards (360-600 $\mu \mathrm{g} / \mathrm{d}$ for 3-7-yearolds). Methods: As part of a larger study to evaluate dietary methods used to assess F exposure in children, 61 healthy 4-6-year-old children were recruited from a fluoridated area in the north-east of England. Two 24-hour urines were collected from each child, with a 7-day gap between each collection to avoid seasonal variation. F concentration of urine samples was measured directly using F-ion-selective electrode (Model 9409, Orion) after adding TISABII. A Paired T-test was used to investigate daily variation in UFE. Results: The mean (SD) UFEs for the 1st and 2nd collections were 370 (202) and 371 (166) $\mu \mathrm{g} /$ day, respectively. No statistically significant differences in the mean UFE between the two collections were detected. However, there was a large within-child dayto-day variation in the rate of UFE, with a 95\% Confidence Interval of 119 to $171 \mu \mathrm{g} /$ day. The daily UFE was below the provisional WHO standards range for $53 \%$ of children. Conclusion: Results indicated that based on the provisional WHO standards, more than half of the children living in a fluoridated area did not receive the recommended intake of $\mathrm{F}$ for effective protection against caries.

Supported by grants from University of Teesside Research Doctoral Scholarships and The Borrow Foundation.

\section{0 \\ Individual Fluoride Exposure History Better Explain Effect of Water Fluoridation than Area-Level Fluoridation Status}

L.G. Do *, A.J. Spencer, K.F. Roberts-Thomson, J. Armfield

loc.do@adelaide.edu.au

Australian Research Centre for Population Oral Health, University of Adelaide, Australia

Background: Recent studies comparing caries experience by fluoridation status of current residence of children often report small or insignificant preventive effects of fluoridation. Aim: to compare the preventive effect of fluoridation measured by individual fluoride exposure and area-level fluoridation status. Methods: Permanent caries experience of 10-15-year-old children in four Australian states was collected. Questionnaire-collected information on residential history since birth by postcodes was matched with databases of fluoride levels in water. Current residence was classified as non-fluoridated or fluoridated (having $0.7+$ ppm fluoride). Percentage of lifetime residence in fluoridated areas, adjusted for actual water usage, was estimated as individual fluoride exposure. This estimate was used to group children into having $0 \%,>0-25 \%,>25-50 \%,>50-75$ and $75+\%$ lifetime exposed to fluoridated water. Multilevel modelling using SAS PROC MIXED adjusted for clustering effect within postcodes. Results: Data of 7,222 children aged 10-15 years were available. Permanent caries experience was strongly age-related. Ageand sex-adjusted mean DMFS: 1.26 (95\%CI: 1.14-1.37). Fluoridation status of current residence explained only small variances in mean DMFS. No statistically significant preventive effect in mean DMFS scores was observed between children currently residing in fluoridated compared to non-fluoridated areas. However, adjusted percentage lifetime exposure to fluoridated water was significantly associated with the preventive effect in mean DMFS. Compared with those having $0 \%$ lifetime exposure, those having $>25-50 \%,>50-75 \%$ or $75+\%$ lifetime exposure had significantly lower age- and sex-adjusted DMFS scores (parameter estimates: $0.21(\mathrm{p}=0.040),-0.289(\mathrm{p}=0.0049)$, and $-0.388(\mathrm{p}=0.0009)$, respectively). Dose-response effect was observed. Conclusion: Individual percentage of lifetime exposure to fluoridated water better captured the preventive effect of fluoridation than simplistic dichotomised fluoridation status of current residence. This should be taken into account when evaluating effectiveness of water fluoridation.

Supported by Australia National Health and Medical Research Council (NHMRC) project grant. Loc Do was supported by an NHMRC Capacity Building Grant. State Dental Services in South Australia, Victoria, Tasmania and Queensland participated in data collection. 


\section{1}

\section{A Systematic Review about the Effectiveness of Toothpaste in Preschool Children}

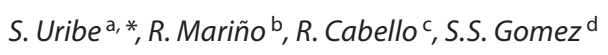

sergiouribe@uach.cl

aSchool of Dentistry, Universidad Austral de Chile, Valdivia,

Chile; ' University of Melbourne, Australia; 'Universidad de Chile,

Santiago, 'Universidad de Valparaiso, Chile

Aim: To systematically review the available evidence about the effectiveness of fluoridated toothpaste (FT) on caries in preschool children. Methods: A systematic search in electronic databases for articles published between 1966 and January 2008 was conducted with the inclusion criteria of a randomized or controlled clinical trial, at least 2 years follow-up and caries increment in the primary (deltadmfs/t) dentition as endpoint. Reviewers individually performed data extraction and scored each study using a quality assessment instrument. We tested for heterogeneity, publication bias, and study quality. We pooled effect sizes (prevented fraction $[\mathrm{PF}]$ ) using fixed-effects and random-effects models. Results: Of 253 studies, seven meet all the inclusion criteria: two systematic reviews and five clinical trials. The studies show differences in caries (PF) from 13 with FT against placebo and 2.6 for 450-550 ppm versus 1,055-1,450 ppm FT. Conclusion: Fluoridated toothpaste is effective against caries in preschool children, with a correlation between fluoride concentration and anticaries effectiveness. More clinical studies are needed to assess the differences in effectiveness between low-high risk children.

Supported by Ministry of Health, Chile.

\section{2}

\section{Selection of the Studies Included in a Meta-Analysis}

S. Tubert-Jeannin ${ }^{\mathrm{a}, *}$, C. Auclair ${ }^{\text {b }}$, P. Tramini ${ }^{\text {C }}$, E. Amsallem ${ }^{\text {d, }}$ A.G. Schulte ${ }^{\mathrm{e}}$, L. Gerbaud $^{\mathrm{f}}$

stephanie.tubert@u-clermont1.fr

${ }^{a}$ Faculty of Dentistry, University of Auvergne, bPublic Health, CHU of Clermont-Ferrand, ' Faculty of Dentistry, University of Montpellier, ${ }^{\mathrm{d}}$ Quality - Evaluation (CETAF), Saint-Etienne, France; ${ }^{\text {FFaculty }}$ of Dentistry, University of Heidelberg,

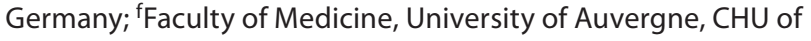

Clermont-Ferrand, France

The conduct of a meta-analysis involves the careful application of a strict protocol in order to ensure the validity of the results. Search methods for identification of studies, criteria for considering studies to be included, methods used for data collection and analysis need to be defined precisely. The process of selection of the studies included in a meta-analysis is very restrictive: a small proportion of the reports found during the search are selected for inclusion in the review. Many reports are irrelevant or do not fulfil the criteria for selection in the meta-analysis. Many studies are excluded due to a lack of information, the absence of pertinent data or to methodological problems. This can be illustrated by exploring the process of selection of the studies included in the meta-analysis on the efficacy of fluoride supple- ments for preventing dental caries in children. (Tubert-Jeannin S et al, Cochrane Database of Systematic Reviews 2009, Issue 1). In this review, randomised controlled trials with a duration of at least 2 years of ingestion of fluoride supplements were compared with no treatment or with other preventive measures such as topical fluorides in children less than 16 years. Studies without caries assessment were excluded. Following the removal of duplicates, the searches yielded 1,380 records. They were checked on the basis of the title, keywords and abstract. Records which obviously did not refer to the use of fluoride supplements, reported results of in vitro studies, reviews or epidemiological studies were excluded. This led 85 reports for further assessment. Thirty-four reports were excluded for the following reasons: no control group, design not prospective, supplements administered to controls, evaluation after the end of intervention, controls historical or of insufficient duration. A thorough examination of the remaining manuscripts led to the exclusion of 33 more reports. In 6 studies, the study groups were inadequately described. In 7 studies, there was no clear indication of a randomisation process. In 5 studies, baseline evaluation of dental status was lacking. Two studies were excluded due to an insufficient length of follow up. Eight studies were excluded due to a lack of information and absence of many data. Finally, a total of 18 reports were eligible for inclusion in the review.

\section{3 \\ Effect of Two Different Concentrations of Fluoridated Milk on Enamel Remineralisation in situ \\ M. Malinowski *, M.S. Duggal, S.M. Strafford, K.J. Toumba \\ M.Malinowski@Leeds.ac.uk \\ Paediatric Dentistry, Leeds Dental Institute, UK}

The aim was to measure the ability of 2.5 and $5.0 \mathrm{ppm}$ fluoride (F) in milk to promote remineralisation of pre-demineralised enamel in an in situ model with a cariogenic challenge. 25 subjects were recruited to a controlled, randomised, 3-arm crossover, double-blinded clinical study. Subjects wore an intra-oral lower removable appliance containing enamel slabs with artificial caries lesions, continuously for 21 days during each study arm. During the entire study subjects used fluoride-free toothpaste. The cariogenic challenge comprised 5 dippings (each 2 min duration) per day in $12 \%$ sucrose solution. In addition, subjects dipped their appliances in $50 \mathrm{ml} \mathrm{milk}$, either with no added fluoride (negative control), or with 2.5 or 5.0 ppm F for 5 min twice daily during the test period. Subjects also drank $100 \mathrm{ml}$ twice per day of the same milk immediately on reinserting their appliances in order to replicate the topical and systemic effects of fluoridated milk. At the end of the study, mineral loss of the artificial caries lesions of the slabs was measured using Transverse Microradiography (TMR) to assess changes in mineralisation. 23 subjects completed the study. Although both fluoride concentrations and the non-fluoride control milk were effective in promoting statistically significant remineralisation $(\mathrm{p}<0.005)$ compared with their respective baseline values, a higher value was observed for $2.5 \mathrm{ppm}(697.84$ \pm 388.06), compared with $5.0 \mathrm{ppm} \mathrm{F}(499.98 \pm 357.47)$ and $0 \mathrm{ppm}$ F (403.58 \pm 413.78). However, the differences were not statistically significant when compared between groups. In conclusion, 
using a caries- like in situ model and a randomised controlled double blind cross over study design, conducted to $\mathrm{GCP} / \mathrm{ICH}$ guidelines, it seems clear that a concentration of 2.5 and $5.0 \mathrm{ppm}$ fluoride in milk significantly promoted enamel remineralisation. Supported by a grant from The Borrow Foundation.

\section{4}

Caries-Preventive Efficacy of Silver Diamine Fluoride and Sealant Application in a School-Based Daily Fluoride Toothbrushing Program

\author{
W.H. van Palenstein Helderman ${ }^{a}{ }^{*}, B$. Monse ${ }^{b}$, \\ R. Heinrich-Weltzien ${ }^{c}$, C.J. Holmgren ${ }^{a}$ \\ w.vanpalenstein@dent.umcn.nl \\ a Radboud University Nijmegen Medical Centre, \\ The Netherlands; ${ }^{\text {b}}$ Department of Education, Health and \\ Nutrition Centre, Cagayan de Oro, Philippines; ' $D e p a r t m e n t ~ o f$ \\ Preventive Dentistry and Paediatric Dentistry, University Hospital \\ of Jena, Germany
}

Rationale: As caries remains largely untreated in children in low- and middle-income countries, fundamental components of school-based oral health programs are exposure to fluoride and low cost approaches to prevent onset of caries on the occlusal surface of the first molars. Objective: To assess the effectiveness of a one time application of $38 \%$ SDF and to compare it with ART sealants in preventing the onset of caries in permanent first molars. Methods: Prospective controlled clinical trial including 1,155 first graders, $6-8$ years old, in 8 public elementary schools with a daily fluoride toothbrushing program in the Philippines. Two schools were randomly selected where the children did not receive treatment. Children of the remaining schools received either a one time SDF application or an ART sealant on their erupted permanent first molars. Non-compliance to the daily school-based toothbrushing scheme in three schools offered the opportunity to analyse the caries preventive effect of SDF and ART sealants separately in toothbrushing and in non-toothbrushing children. Results: Daily school-based fluoride toothbrushing reduced caries increment statistically significantly by $39 \%$ and caries progression into the pulp by $61 \%$. In the toothbrushing children, a one time SDF application did not reduce caries increment whereas ART sealants reduced the onset of caries. Conclusion: The results emphasise the high efficacy of daily school based fluoride toothbrushing. SDF application as a low cost intervention was not proven to be an effective additional strategy in the prevention of caries, if children are exposed to daily fluoride toothpaste.

3MESPE for supplying the glass ionomer material used in the study and CIM/GTZ for financing other consumable materials and travel. 


\section{Caries Research}

\section{Session 5 \\ Microbiology}

\section{5}

\section{Anticariogenic Effects of Oenothera biennis Seed Extracts on Streptococcus mutans Activity}

\author{
M. Matsumoto-Nakano a, , K. Nagayama a , K. Fujita ${ }^{a}$, S. Inagaki ${ }^{\text {a }}$,
} S. Kawabata ${ }^{\text {b }}$, T. Ooshima ${ }^{\text {a }}$

ooshima@dent.osaka-u.ac.jp

a Department of Pediatric Dentistry, and ${ }^{\text {b}}$ Department of Oral and Molecular Microbiology, Osaka University Graduate School of Dentistry, Osaka, Japan

Streptococcus mutans, implicated as a primary causative agent of dental caries, adsorbs into an acquired pellicle formed on the enamel surface with salivary proteins through hydrophobic action at the initial stage of the disease. Next, the organism synthesizes adhesive glucans from sucrose by the action of glucosyltransferases (GTFs) and those glucans mediate the firm adherence of bacterial cells to tooth surfaces. Several kinds of polyphenols have been widely reported to have anticariogenic activities, with Oenothera biennis seed extract (OBSE), from evening primrose, shown to contain such polyphenols. In the present study, we examined the inhibitory effects of polyphenols contained in OBSE on caries development. In vitro analyses showed that OBSE had a strong aggregation activity toward S. mutans and reduced its hydrophobicity. Furthermore, the rates of insoluble glucan synthesis by recombinant GTFB (rGTFB) and sucrose-dependent adherence of $S$. mutans were decreased by the addition of OBSE. In experiments with specific pathogen-free (SPF) SpragueDawley rats, animals infected with $S$. mutans MT8148R given in drinking water showed significantly lower caries scores than those without OBSE. Taken together, our results indicate that OBSE is a possible anticariogenic agent.

Supported in part by the Fund for Scientific Promotion of Kobayashi Pharmaceutical Co., Ltd.

\section{6 \\ Contribution of Collagen-Binding Adhesin of Streptococcus mutans to Deterioration of Intracerebral Hemorrhage \\ R. Nomura ${ }^{\mathrm{a}, *}$, K. Nakano ${ }^{a}$, N. Taniguchi ${ }^{\mathrm{a}}$, K. Wada $^{\mathrm{b}}$, M. Matsumoto ${ }^{\text {a }}$ T. Ooshima ${ }^{\text {a }}$ \\ ooshima@dent.osaka-u.ac.jp \\ aDepartment of Pediatric Dentistry, and bepartment of Pharmacology, Osaka University Graduate School of Dentistry, Osaka, Japan}

Streptococcus mutans, a major pathogen of dental caries, is occasionally isolated from tissues outside the oral cavity. However, the relationship between $S$. mutans and systemic diseases other than infective endocarditis (IE) remains to be elucidated. Recently, an approximately $120-\mathrm{kDa} \mathrm{Cnm}$ protein that binds to type I collagen was identified as a cell-surface antigen of $S$. mutans and strains with the protein were shown to have a prevalence of approximately $10-20 \%$. Such $S$. mutans strains are considered to have advantages for attaching to impaired heart valve tissue, which can lead to the onset of IE. In the present study, we analyzed the correlation between $S$. mutans strains with collagen-binding properties and intracerebral hemorrhage (ICH), a major complication of IE. Isogenic mutant strains with a defect of $\mathrm{Cnm}$ were constructed from $S$. mutans strains with the $\mathrm{cnm}$ gene by insertional inactivation of an erythromycin resistant gene. Our results showed that those cnm-inactivated mutant strains lacked collagen-binding properties. Evaluation of ICH model mice with photochemical impairment of the middle cerebral artery revealed that infection with TW295 (cnm-positive) from the jugular vein induced significantly greater hemorrhage areas than seen in those infected with the oral isolate MT8148 (cnm-negative) or TW295CND (cnm-inactivated isogenic mutant strain constructed from TW295). In addition, analyses of other clinical strains with various collagen-binding activities showed a positive correlation between collagen-binding intensity and size of the hemorrhage area. Furthermore, a negative correlation was identified between collagen-binding intensity and platelet aggregation rates. These results indicate that the $\mathrm{Cnm}$ protein of $S$. mutans is an important virulence factor for deterioration of ICH.

Supported by the Grants-in-Aid for Scientific Research (A) 19209063 and Grant-in-Aid for Young Scientists (A) 21689052 and (B) 21792067 from the Ministry of Education, Culture, Sports, Science and Technology of Japan. 


\section{7 \\ Viability and Mechanisms of Herbal Tooth Powder on Cariogenic Streptococcus mutans}

R. Karthikeyan, B.T. Amaechi *

amaechi@uthscsa.edu

University of Texas Health Science Center, San Antonio, USA

Introduction: Cariogenic biofilm formation is a biological process involving attachment and proliferation of oral bacteria, such as Streptococcus mutans, on the tooth surface. S. mutans is considered the main bacteria involved in the etiology of dental caries. The aim of the present study was to investigate the effectiveness of extract from Indian herbal tooth powder (Pita karuan; Mark Tierno's Medicinal herbs products) against S. mutans. Methods: S. mutans biofilm was grown on Lab-Tek chamber slides with cover slide. The grown biofilm was treated with ethanol-extracted plant extract for $1 \mathrm{~min}, 5 \mathrm{~min}$ and $1 \mathrm{~h}$, and then stained using LIVE/DEAD ${ }^{\circledR}$ BacLight ${ }^{\mathrm{TM}}$ Bacterial Viability Kit to test the viability of $S$. mutans. Chlorhexidine gluconate mouthrinse served as the control treatment. Following staining, the biofilm samples were examined via an Olympus FV1000 confocal laser scanning system on an IX81 microscope at excitation wavelengths of 488 and $543 \mathrm{~nm}$. Effectiveness and mechanism was tested by structural analysis by Variable Pressure Digital Scanning electron microscope (SEM) imaging of biofilm formed overnight in glass slides after treatment with Minimum Inhibitory Concentration of plant extract for $30 \mathrm{~min}$ at room temperature. Results: For biofilm treated with plant extract, viability live/dead staining showed significant $(\mathrm{p}<0.05)$ increase in dead cell areas at all time points from $53.6 \%$ in $1 \mathrm{~min}$ to $85 \%$ at $1 \mathrm{~h}$. Chlorhexidine showed only $28 \%$ in $1 \mathrm{~min}$, approximately one half of the activity of plant extract. SEM images showed damage to cell membranes and cell walls of $S$. mutans by plant extract. Conclusion: The higher and faster killing activity suggests that plant extract has effective antibacterial activity against $S$. mutans, and may be a useful medication inhibition of cariogenic biofilm.

\section{8 \\ Comparison of Long-Term Microcosm Biofilms Formed on Different Substrata}

K. Tomiyama *, R.A.M. Exterkate, J.M. ten Cate

tomiyma@kdcnet.ac.jp

Department of Cariology, Endodontology, Pedodontology,

Academic Centre for Dentistry Amsterdam (ACTA),

The Netherlands

This study aimed to investigate microcosm biofilm formation during extended periods on different substrata. A high-through put model was used based on active attachment of bacteria to surfaces. Smooth surface of cylindrical specimens were obtained from bovine lower incisors. In this study, dentine specimens and circular glass cover slips were used as substrata to grow biofilms. Saliva was obtained from a single donor, diluted 50 times and was then used to inoculate both dentine and glass specimens. Experiments were performed anaerobically in McBain medium, which was refreshed twice daily. Biofilm specimens were recovered after 48, 120, 144,
168 and $192 \mathrm{~h}$, and assessed for cfu counts and lactic acid production capacity. Also, the $\mathrm{pH}$ and calcium release in the spent medium were analyzed. Results show constant calcium loss during the period for the dentine specimens and an about one unit $\mathrm{pH}$ difference in the mediums between the two substrata types (dentine $\mathrm{pH} 5.24$ versus glass $\mathrm{pH}$ 4.15). Biofilms on the two substrata show different temporal patterns regarding cfu counts and acid formation. Acid formation and cfu counts increased with time in the glass group, but remained constant for the dentine group. In conclusion, it was possible to form stable, mature biofilms during longer periods. These biofilms differed in important properties, and hence presumably composition, depending on the choice of substratum.

\section{9 \\ Streptococcus mutans and Streptococci Distribution in Plaque Biofilm Formed on Enamel with Glass-Ionomer Cement Restoration}

T.T. Thuy ${ }^{\text {a, }}$, H. Nakagaki a , K. Kato a , Y. Kawamura ${ }^{\text {b }}$, C. Robinson ${ }^{c}$

thranthuthuyrhm@yahoo.com

${ }^{a}$ School of Dentistry, and ${ }^{b}$ School of Pharmacy, Aichi-Gakuin University, Nagoya, Japan; 'Division of Oral Biology, Leeds

Dental Institute, University of Leeds, UK

Previous studies show fluoride released from dental materials assist in the remineralization of surrounding dental tissues. It was reported that glass-ionomer cements (GICs) have antimicrobial properties. However antimicrobial effects of fluoride releasing materials in vivo remain contentious. Recent microbiological studies have prompted an intensified awareness of the important of streptococci other than mutans streptococci to dental caries. This study examined the distribution of Streptococcus mutans and streptococci within dental biofilms formed in vivo. Biomass was collected by plaque generation devices set on upper molars of twenty consenting volunteers. Plaque biofilms were allowed to develop on sound enamel (Enamel group) and enamel with GIC (Fuji VII, GC) (GIC group) during the period of 7 days. Biofilms were then sectioned throughout their depth. Sections stained with neutral toluidine blue were used for evaluation of biomass volume. Genomic DNA was extracted from remaining sections (QIAamp DNA Mini Kit, Qiagen). S. mutans and streptococci were quantified by TaqMan real-time PCR (7900HT, Applied Biosystems). The volume occupied by biomass and biomass profile were similar in the two groups. The level of bacteria varied widely among subjects. No difference was found between groups in both number and distribution of $S$. mutans. There was no significant difference in the level of streptococci between groups. However the distribution of oral streptococci in GIC group revealed significant changes. Within $300 \mu \mathrm{m}$ biomass depth toward enamel interface, the level of streptococci were significant lower in the first layer closed to enamel surface compared with the subsequent layers only in GIC group (Wilcoxon test, $\mathrm{p}<0.005$ ) and not in Enamel group. It can be suggested that the environment closed to GIC restorations is not habitats favorable for the growth of oral streptococci.

This work was supported by the AGU High-Tech Research Center Projects from the Ministry of Education, Culture, Sport, Science and Technology, Japan and the Overseas Training Projects from the Ministry of Education and Training, Viet Nam. 
90

\section{Screening of Plant Extracts from Brazilian Pantanal for Antimicrobial Activity against Bacteria with Cariogenic Relevance}

F.L. Brighenti ${ }^{a}$, ${ }^{*}$ M.J. Salvador ${ }^{\text {b }}$, A.C.B. Delbem ${ }^{\text {c }}$, L.S.F. Freitas ${ }^{\text {a }}$, C.Y. Koga-Ito ${ }^{\text {a }}$

ferbrighenti@gmail.com

aUNESP, Universidade Estadual Paulista, São José dos Campos,

bUnicamp, Universidade de Campinas, 'UNESP, Universidade

Estadual Paulista, Aracatuba, Brazil

In the past decades, interest on natural products has increased. This study aimed to select plants extracts from Pantanal with potential anticariogenic properties. The plants studied were: Jatropha weddelliana, Scheelea phalerata, Buchenavia tomentosa, Croton doctoris, Mouriri elliptica, Mascagnia benthamiana, Senna aculeata, Unonopis lindmanii, Allagoptera leucocalyx and Bactris glaucescens. Thirty extracts (20 g of dried leaves in $400 \mathrm{ml}$ of deionized water) were obtained using different times and temperatures (A: $5^{\prime} / 100^{\circ} \mathrm{C}$; B: $1 \mathrm{~h} / 55^{\circ} \mathrm{C}$; $\mathrm{C}: 72 \mathrm{~h} /$ room temperature). The extracts were liophilyzed and re-suspended in BHI broth before use. Plants were screened for antibacterial activity at $50 \mathrm{mg} / \mathrm{ml}$ using the agar well diffusion test against Actinomyces naeslundi ATCC 19039, Lactobacillus acidophilus ATCC 4356, Streptococcus mutans ATCC 35688, S. sanguinis ATCC 10556 and S. sobrinus ATCC 33478. The active extracts were then tested regarding their minimum inhibitory concentration (MIC) and minimum bactericidal concentration (MBC). Twenty-one extracts (70\%) were active against at least one microorganism. S. mutans and L. acidophilus showed fewer active extracts, smaller inhibition halo (9-13 $\mathrm{mm})$ and higher MIC (1.56-6.25 $\mathrm{mg} / \mathrm{ml})$ and MBC values $(12.5$ up to higher than $50 \mathrm{mg} / \mathrm{ml}$ ) in comparison to the other microorganisms studied. S. sanguinis and $A$. naeslundii were the most susceptible, showing inhibition halo higher than $40 \mathrm{~mm}$ in some extracts. The lowest MIC values for S. sanguinis, A. naeslundii and S. sobrinus were $0.39,0.78$ and $0.39 \mathrm{mg} / \mathrm{ml}$, respectively. For MBC, the lowest values found were 3.13 and $1.56 \mathrm{mg} / \mathrm{ml}$, for S. sanguinis and $A$. naeslundii, respectively. No MBC was observed for extracts tested against $S$. sobrinus. A wide variety on extract activity was found for the microorganisms studied and extraction method. Some of the extracts studied may have promising anticariogenic activity.

Funding: FAPESP (08/04114-2 and 08/53299-5).

\section{1 \\ Analysis of Streptococcus mutans Biofilms of Genotypes Isolated from Dental Biofilm under Sucrose Exposure}

J.A. Cury*, R.A. Arthur, R.A. Ccahuana-Vásquez, C.P.M. Tabchoury

jcury@fop.unicamp.br

Piracicaba Dental School, UNICAMP, Piracicaba, SP, Brazil

S. mutans genotypes isolated from dental biofilm formed under sucrose exposure could have more cariogenic properties than those isolated in the absence of sugar pressure, what was evalu- ated in the present study. Eight distinct genotypes, from 3-day in situ plaque-like biofilm formed in the presence or absence of $20 \%$ sucrose solution exposure $8 \times /$ day, which also showed different acidogenicity and aciduricity traits when tested in planktonic conditions, were grown as biofilm on enamel slabs. The biofilms $(\mathrm{n}=3)$ were formed on human saliva coated bovine enamel slabs, whose surface hardness ( $\mathrm{SH}$ ) were pre- determined. The biofilms were grown at $37^{\circ} \mathrm{C}, 5 \% \mathrm{CO} 2$ in ultrafiltered tryptone-yeast extract broth containing $0.10 \mathrm{~mm}$ glucose and additionally they were subjected $8 \times /$ day for $1 \mathrm{~min}$ to $20 \%$ sucrose solution. The medium was changed at $24 \mathrm{~h}$ and sucrose every time. On the 5 th day the biofilms were collected for biomass (Colony forming units, CFU) and insoluble (IEPS) and soluble extracellular (SEPS) polysaccharides determination. Enamel $\mathrm{SH}$ was again determined and the percentage of SH loss (\%SHL) was calculated. The genotypes were statistically distinct $(\mathrm{p}<0.05)$ only concerning biomass (from 0.4 to $1.4 \mathrm{CFU} \times 109 / \mathrm{mg}$ biofilm dry weight) and enamel demineralization (from 26.7 to $49.7 \% \mathrm{SHL}$ ) irrespective if they were isolated from dental biofilm formed under sucrose exposure or not. No differences $(p>0.05)$ were found regarding concentration of either IEPS or SEPS (mean \pm SD of $47.1 \pm 9.0$ and $24.7 \pm 5.1 \mu \mathrm{g} / \mathrm{mg}$ biofilm dry weight, respectively). The findings suggest that $S$. mutans genotypes isolated from dental biofilm formed under sucrose pressure are not more cariogenic than those isolated in the absence of sugar exposure.

Supported by FAPESP (Proc. 03/10972-8, 05/03089-6 and 07/08000-9).

\section{2}

Effect of а к-Casein Peptide on Streptococcus mutans Biofilms

S.G. Dashper*, S.-W. Liu, E.C. Reynolds

stuartgd@unimelb.edu.au

Oral Health Cooperative Research Centre, The University of Melbourne, Australia

Streptococcus mutans is considered as one the major causative agents of dental caries and this bacterium exists as part of dense polymicrobial biofilms on the tooth surface. The aim of this study was to determine the effect of $\kappa$-casein(106-169) on a S. mutans biofilm and compare its effects with the widely used antimicrobial agent chlorhexidine. S. mutans biofilms were cultured in 3-channel flow cells using an artificial salivary medium. After 16 $h$ of incubation the cells were stained and imaged using confocal laser scanning microscopy to reveal structured S. mutans biofilms with an average depth of $14.8 \pm 5.9 \mu \mathrm{m}$ and a biovolume of $9.2 \pm 3.5 \mu \mathrm{m}^{3} / \mu \mathrm{m}^{2}$ of substratum. A $10 \mathrm{mg} / \mathrm{ml}$ preparation containing $\mathrm{\kappa}$-casein(106-169) and $20 \mathrm{mM} \mathrm{ZnCl}_{2}$ was applied to the 16 h S. mutans biofilms for $10 \mathrm{~min}$ and this treatment reduced biofilm biovolume to $1.7 \pm 0.6 \mu \mathrm{m}^{3} / \mu \mathrm{m}^{2}(-76 \%)$ and average biofilm thickness to $2.3 \pm 1.1 \mu \mathrm{m}(-78 \%)$. Chlorhexidine $(0.1 \%)$ treatment reduced biofilm biovolume to $3.2 \pm 0.7 \mu \mathrm{m}^{3} / \mu \mathrm{m}^{2}(-66 \%)$ and average thickness to $4.7 \pm 0.8 \mu \mathrm{m}(-69 \%)$. There were statistically significant differences between biometric parameters of the control, $\kappa$-casein and chlorhexidine treatments $(\mathrm{p}<0.05)$, indicating that the $\kappa$-casein(106-169) preparation was more ef- 
ficacious at disrupting established S. mutans biofilms. However chlorhexidine was significantly more effective than the $\kappa$-casein (106-169) preparation when tested against $6 \mathrm{~h} \mathrm{~S}$. mutans biofilms that were approximately half the thickness of the $16 \mathrm{~h}$ biofilms. In conclusion chlorhexidine appeared to kill bacteria at the biofilm surface but penetrated these biofilms poorly whereas the $\kappa$-casein (106-169) preparation significantly disrupted mature biofilm structure.

Supported by the Australian Government's Cooperative Research Centres Programme.

\section{3}

Ecological Importance of Lactobacillus rhamnosus for Caries Progression in Early Childhood Caries

\author{
A. Callaway ${ }^{\mathrm{a}, *}$, A. Borutta ${ }^{\mathrm{b}}$, F. Schmidt ${ }^{\mathrm{c}}$, H. Küpper ${ }^{\mathrm{b}}$, \\ B. Willershausen ${ }^{\text {a }}$ S. Kneist ${ }^{\text {b }}$ \\ callaway@uni-mainz.de \\ aUniversity of Mainz, Dental School, bUniversity of Jena, Dental \\ School, 'University of Greifswald, Interfaculty Institute of \\ Genomics and Functional Genomics, Germany
}

Lactobacilli are considered as secondary invaders of existing carious lesions and specialists for caries progression. In a previous study it could be shown that L. paracasei ss paracasei, L. paracase $i$ ss tolerans, L. rhamnosus, L. gasseri, and L. alimentarius were found sporadically in all caries progression stages of deciduous molars (75/85) with Caries profunda, and that L. paracasei ss paracasei and $L$. rhamnosus might be the specialists among the lactobacilli in the caries progression [Kneist et al.: Caries Res 2008; $42: 218]$. The aim of the present study was to characterize lactobacilli from carious dentine of 10 deciduous teeth of children with early childhood caries. From carious dentine $(2 \times 51,3 \times 61,54$, $55,64,84,85) 62$ isolates, grown on Rogosa agar and presumed to be lactobacilli, were identified by physiological and biochemical characteristics and shotgun mass mapping (SMM) based on determining mass spectra profiles by MALDI-TOF mass spectrometry of trypsinated isolates and database comparison with mass spectra from 20 reference strains. The mean total viable count of lactobacilli in carious dentine was determined with $\log _{10} 2.3449$. The majority of the 62 isolates were identified as L. rhamnosus, two as $L$. case $i$, one isolate was an obligate heterofermentative species, which couldn't be identified. L. rhamnosus reached in 7 teeth a percentage of $>70 \%$ of the total viable count of the lactobacilli, and was thus the dominant species in the carious dentine. L. casei was found in only one tooth, with a prevalence of $1 \%$ of the lactobacillus cfu. In agreement with the previous study it could be shown that L. rhamnosus may be the specialist in the carious progression of early childhood caries.

Supported by Universities in Mainz and Jena.

\section{4 \\ Microbiological and Salivary Cariogenic Features of Pediatric Patients with Sickle Cell Anemia}

\author{
B.M. Matos ${ }^{a}{ }^{*}$, Z.E.A. Ribeiro ${ }^{\text {a }}$, E.Y. Komiyama ${ }^{a}$, M.S. Figueiredo ${ }^{b}$, \\ J.A.P. Braga ${ }^{\text {b }}$, C.Y. Koga-Ito ${ }^{a}$ \\ mellodematos@yahoo.com.br \\ aSão José dos Campos Dental School, baulista School of \\ Medicine, São Paulo, Brazil
}

Sickle cell anemia is the most frequent hereditary disease in Brazil. Children with this disease are under a long-term prophylactic treatment with penicillin and little is known about the effect of this therapy on oral microbiota. The aim of this study was evaluate the influence of penicillin prophylaxis on microbiological (mutans streptococci, lactobacilli and yeasts) and salivary (salivary flow rate and buffering capacity) cariogenic features of pediatric patients with sickle cell anemia. Forty children (4-11 years old) with sickle cell anemia (genotype SS) under a long-term prophylactic treatment with penicillin (at least for 6 months) were included in the study. A control group of healthy children with matching age was also included. Stimulated saliva was collected for $5 \mathrm{~min}$ and $\mathrm{dmft} / \mathrm{DMFT}$ scores were performed. Salivary flow rate and buffering capacity were determined. Aliquots of saliva were plated in duplicate on selective media to mutans streptococci (mitis salivarius bacitracin sucrose agar), lactobacilli (Rogosa agar with acetic acid) and yeasts (Sabouraud dextrose agar with chloramphenicol) and incubated. The results showed statistical differences to counts of yeasts (Mann-Whitney test, $\mathrm{p}=0.0027$ ) and buffering capacity (Student's t test, $\mathrm{p}=0.0480$ ). There were no statistical differences to mutans streptococci and lactobacilli counts and to salivary flow tests $(\mathrm{p}>0.05)$. The mean $\mathrm{dmft}$ and DMFT were respectively, 2.13 and 1.60 for the sickle cell anemia group, and 2.38 and 1.30 for the control group, but there were no statistical differences between the groups studied (Mann-Whitney test, $p>0.05$ ). We could conclude that pediatric patients with sickle cell anemia showed higher levels of yeasts and lower buffering capacity than the control group.

Supported by São Paulo Research Foundation (FAPESP) Process 2007/58999-2.

\section{5 \\ Caries Activity in Plaque Grown on Sealants: A Human Saliva-Based in vitro Study}

\section{Hauke, H. Stöger, B. Schmid, I. Häberlein * \\ ingo.haeberlein@mmm.com \\ Dental Biotechnology Labs, 3M ESPE, Seefeld, Germany}

Dental sealants are known to be effective in preventing pit and fissure caries. It has been shown that ClinproTM Sealant prevents completely progression of artificial enamel lesions in an in-situ caries model [Paris et al.: Caries Research 2009; 43: 228]. Aim of the study was to investigate if the protective effect of dental sealants is solely due to the formed mechanical barrier or if, in addition, sealants lower the caries activity in plaque. Human saliva was used to grow plaque on materials placed per manufacturers' 
IFU on bovine enamel specimens. Plaque growth was initiated via 1:4 mixture of human saliva and MCM-sucrose-culture media for $4 \mathrm{~h} / 37^{\circ} \mathrm{C}$; the media was replenished for the next $21 \mathrm{~h} / 37^{\circ} \mathrm{C}$. The plaque was collected and lyophilized to determine plaque biomass. Exposed surface area of each specimen was measured. Activity of caries bacteria prevailing in plaque was determined via the amount of released lactic acid, which was measured with ClinproTM Cario L-PopTM (3M ESPE). Plaque grown on bovine enamel showed lactic acid release of $2.15 \mathrm{mmol} / \mathrm{mg} \cdot \mathrm{cm}^{2} \cdot \mathrm{h}$. Plaque grown on all investigated sealants showed less lactic acid release between 0.40 and $1.24 \mathrm{mmol} / \mathrm{mg} \cdot \mathrm{cm}^{2} \cdot \mathrm{h}$. Anova and 2-Sample T-Test revealed that only ClinproTM Sealant (3M ESPE) achieved a statistically significant difference to bovine enamel $(p<0.05)$ whereas ClinproTM Sealant showed strongest control over caries activity in plaque. The data reveals that sealants have the potential to lower the caries activity in the plaque layer grown on top of the sealants.

\section{6 \\ Influence of Sweetened Gum Chewing on Profiles of Biomass Volume and Glucan within Dental Plaque}

K. Kato *, K. Tamura, S. Sakakibara, Y. Ou, H. Nakagaki

kazkato@dpc.aichi-gakuin.ac.jp

School of Dentistry, Aichi-Gakuin University, Nagoya, Japan

This crossover trial was carried out to estimate the influence of sucrose and xylitol in chewing gum on density of biomass volume and glucan throughout plaque using a depth-specific analysis. Fourteen consenting subjects (18-44 years) wore in situ plaque generating devices on their upper molars. They were asked to form plaque by abstaining from tooth brushing for 4 days, chewing sucrose or xylitol gum 6 times daily (total $15 \mathrm{~g}$ ). The samples were separated into $4-6$ layered fractions ( $180 \mu \mathrm{m}$ thick) by serial sectioning ( 2 at $2 \mu \mathrm{m}, 2$ at $4 \mu \mathrm{m}$, then 6 at $4 \mu \mathrm{m}$ ) using a microslicing technique. Thinner sections were stained and used for area measurement to evaluate sample volume. Genomic DNA was extracted from middle thick sections to amplify the 16S rRNA gene sequences by PCR with $S$. mutans-specific primers. Thicker sections were heated with sulfuric acid to produce hydrolysate, in which glucose concentrations were determined to estimate the total amount of glucans. Glucan concentrations (mean $\pm \mathrm{SD} ; \mu \mathrm{g} /$ $\mathrm{mm}^{3}$ ) per layer of the plaque exposed to sucrose and xylitol were $7.25 \pm 4.26$ and $7.48 \pm 5.12$, respectively. Volume occupied by biomass (mean \pm SD; \%) were $36.0 \pm 9.9$ and $33.7 \pm 10.9$. Biomass volume in both groups had a central peak decreasing towards the interior, indicating that the innermost layer of plaque exposed to xylitol possessed a significantly lower biomass. Significant relationships were found between the amount of glucose and biomass in layers of both plaque samples, although frequencies of layer positive for the bacteria, 63.6\% (xylitol) and 94.0\% (sucrose) indicated no significant differences in those indicators. This crossover trial using two kinds of gum showed only limited effects on plaque biofilm structure and matrix composition.

Supported by KAKENHI (C) No. 20592477, Japan.

\section{7 \\ Differences of Growth in Xylitol-Resistant \\ Streptococcus mutans Exposed to Mixed Carbohydrates}

\section{S.Y. Chung *, Y.E. Lee, J.H. Kim, Y.H. Choi, K.B. Song}

kbsong@knu.ac.kr

School of Dentistry at Kyungpook National University, Daegu, South Korea

Streptococcus mutans is one of the main pathogenic bacterium associated with dental caries. Xylitol has been widely used as an effective caries preventive sugar substitute. It is also found that long-term xylitol consumption leads to the emergence of xylitolresistant (XR) mutans streptococci in human. The aim of this study was to identify fundamental characteristics of XR S. mutans and to examine cell growth of XR S. mutans in comparison with those of xylitol-sensitive (XS) exposed to mixed carbohydrate environment. Methods: S. mutans KCTC 3065 was used in this study. Ability of XR strains to grow and produce acid, and virulent factors related to extracellular polysaccharide (ECP) synthesis were monitored. XS and XR strains were cultured in four carbohydrate conditions; glucose based TYE medium (g-TYE), g-TYE plus sucrose, g-TYE plus fructose and g-TYE plus maltose. Two-way ANOVA was performed to test the effect of types of S. mutans and various carbohydrates on cell growth across strains. Results Final OD600 and pH of XR strains were not affected by xylitol treatment. Also, gtf gene expression and ECP synthesis reduced in XR compared to XS. Final growth of both strains was lower in g-TYE plus sucrose and fructose yet higher in g-TYE plus maltose than g-TYE. Changes in the extent of final growth of XR in comparison with that of XS were significant when exposed to g-TYE plus fructose $(p<0.05)$. Conclusions A certain condition of different mixed carbohydrates significantly influenced the growth rate of XR compared to those of XS. This finding may suggests that metabolism of XR S. mutans exposed to fructose is needed to consider other sugar metabolites such as gene expression related to ECP synthesis.

This work was partially supported by National Research Foundation of Korea Grant funded by the Korean Government (KRF-2008-521-E00132) and Korea Caries Prevention Association.

\section{8 \\ Control of Caries Activity in Plaque by Coating Materials \\ R.P. Rusin ${ }^{\text {a, }}$, H. Stöger ${ }^{\text {a }}$ M. Hauke ${ }^{\text {a }, ~ B . ~ S c h m i d ~}{ }^{\text {a }}$, H. Häberlein ${ }^{\text {a }}$, D. Tantbirojn ${ }^{\mathrm{b}}$ \\ rprusin@mmm.com \\ a3M ESPE; bUniversity of Minnesota, USA}

Aim: Characterize (a) caries activity in human-saliva-derived plaque grown on materials, (b) artificial caries inhibition of two coating materials: $3 \mathrm{M}^{\mathrm{TM}}$ ESPE ${ }^{\mathrm{TM}}$ Vanish ${ }^{\mathrm{TM}}$ XT Extended Contact Varnish (VXT), and $3 \mathrm{M}^{\mathrm{TM}} \mathrm{ESPE}^{\mathrm{TM}} \mathrm{Clinpro}^{\mathrm{TM}}$ Sealant (CPS). Experimental Approach: Plaque activity: plaque was grown using 
human saliva on materials placed per manufacturers' instructions on bovine enamel. Plaque growth was initiated with 1:4 mixture of human saliva and MCM-sucrose-culture media $4 \mathrm{~h} /$ $37^{\circ} \mathrm{C}$; media was replenished for the next $21 \mathrm{~h} / 37^{\circ} \mathrm{C}$. Plaque was collected and lyophilized to determine plaque biomass. Exposed surface area of each specimen was measured. Caries bacteria activity in plaque was determined via amount of released lactic acid measured with Clinpro ${ }^{\mathrm{TM}}$ Cario L-Pop ${ }^{\mathrm{TM}}$ (3M ESPE). Cariogenic resistance: using methods of Tantbirojn et al. (Caries Res, 1997), enamel mineral loss $(\Delta \mathrm{Z})$ after simulated cariogenic challenge $\left(0.1 \mathrm{M}\right.$ lactic-acid gel 20 days $\left./ 37^{\circ} \mathrm{C}\right)$ was determined by cross-sectional microhardness under control (nail-varnish), under test coating, and adjacent uncoated enamel 0.5 and $2 \mathrm{~mm}$ from coating. Data were analyzed via one-way ANOVA and Tukey's t test $(\mathrm{p}<0.05)$. Results: Superscripts denote groups that are statistically not different. Plaque biomass $\left(\mathrm{mg} / \mathrm{cm}^{2}\right)$ was: enamel $5.15 \pm$ $1.25^{\mathrm{a}}$, VXT $4.59 \pm 1.09^{\mathrm{a}}$, CPS $4.73 \pm 0.53^{\mathrm{a}}$. Lactic acid release rate $\left(\mathrm{mmol} / \mathrm{mg} \cdot \mathrm{cm}^{2} \cdot \mathrm{h}\right)$ was: enamel $0.99 \pm 0.27^{\mathrm{a}}$, CPS $0.57 \pm 0.21^{\mathrm{b}}$, VXT $0.59 \pm 0.32^{\mathrm{a}}$. Mineral loss $(\Delta \mathrm{Z})$ under the coating $(\operatorname{vol} \% \cdot \mu \mathrm{m})$ was: VXT $79 \pm(137)^{\mathrm{a}}$, CPS $67 \pm(85)^{\mathrm{a}}$; under the control, VXT 200 $\pm(201)^{\mathrm{a}}, 91 \pm(183)^{\mathrm{a}}$; at $0.5 \mathrm{~mm}$ from coating, VXT $249 \pm(344)^{\mathrm{a}}$ CPS $5298 \pm(1,205)^{\mathrm{b}}$; at $2 \mathrm{~mm}$ from coating, VXT $130 \pm(170)^{\mathrm{a}}$, CPS $6783 \pm(1,223)^{\mathrm{b}}$. Conclusions: The statistically significant plaque activity reduction on CPS vs. enamel confirms earlier results (Hauke et al., ORCA 2010). CPS and VXT provided mechanical protection for underlying enamel, and demonstrate a trend toward reduced plaque activity. In addition, VXT demonstrated a statistically significant protective effect at 0.5 and $2 \mathrm{~mm}$ from the coating.

Supported in part by $3 \mathrm{M}$ ESPE.

\section{9 \\ Contrasting Effects of Chemotherapeutic Agents on Plaque Microcosm Biofilm Development and the Cariogenic Microbiota}

C.H. Sissons *, L. Wong, S.K. Filoche, G.M. Wall-Manning, M. van Bekkum

chris.sissons@otago.ac.nz

University of Otago Wellington, New Zealand

Dental plaque is a complex biofilm community that in caries becomes more aciduric acidogenic plaque from carbohydrate-induced acidogenesis. Aim: To evaluate in vitro chemotherapeutics that potentially counter and reverse development of cariogenic plaque. Methods: Plaque microcosms biofilm were developed in the Multiplaque Artificial Mouth with continuous artificial saliva (DMM) and 8-hourly 6-min sucrose pulses. The chemotherapeutics: chlorhexidine (CHX) 0.2\%, Listerine ${ }^{\mathrm{TM}}$ (original), and F101 (a purified extract of Sophora flavescens) at 100 and 1,000 ppm were applied in 4-hourly $15 \mathrm{~min}$ pulses from the beginning of growth, after day three growth, and compared to controls. Treatment stopped on day 11. Culture continued for 12-14 days to evaluate post treatment regrowth and microbiota. Biomass (net weight) accumulation and endpoint microbiota by DGGE and checkerboard analysis were evaluated. Results: CHX strongly inhibited growth. Regrowth of the day 0 -treated plaque was immediate and rapid but the day 3 preformed plaque require $\mathrm{CHX}$ washout. Complex biofilm communities resulted and cariogenic species increased. Listerine was less inhibitory and disruptive to the community composition. Cariogenic species were comparable to control levels or reduced. F101 had opposite effects to $\mathrm{CHX}$. Initially, the biofilm grew for 5 days. Then growth stopped for both day 0 and day 3 treatments and did not resume after treatment stopped. Higher cariogenic streptococci levels resulted. Conclusions: $\mathrm{CHX}$ is probably typical of strong broad-spectum antiseptics. It did not completely kill species but disrupted community interrelationships such that regrowth communities could be more cariogenic. Less community disruption by phytochemicals such as Listerine may modulate plaque development to a less cariogenic state. The unexplained effects of F101 suggest that phytochemicals exist with unexpected and effects and could have potentially useful biofilm community modulating properties.

Supported by Health Research Council of New Zealand, New Zealand Lottery Health Board, Wellington Medical Research Foundation.

\section{0 \\ Photobleaching Phenomena Induced in Oral Biofilm by Quantitative Light-Induced Fluorescence}

C.K. Hope ${ }^{\mathrm{a}, *}$, E. de Josselin de Jong ${ }^{\mathrm{a}, \mathrm{b}}$, S.M. Higham ${ }^{\mathrm{a}}$

C.Hope@liverpool.ac.uk

aUniversity of Liverpool, ' Inspektor Research Systems BV,

Bussum, The Netherlands

Quantitative Light-Induced Fluorescence Digital (QLFD) is an adaptation of QLF which employs a different light source and modified filter set combined with a digital SLR camera. QLFD was developed to enhance the detection and quantification of plaque as revealed by the intrinsic fluorescence of bacterial porphyrins. Since fluorescence intensity is proportional to the incident light intensity, it is prudent to maximise the amount of light in order to likewise maximise the sensitivity and dynamic range of QLFD. However, high irradiance could lead to photobleaching of the fluorophores which would adversely affect the accuracy of the system and the extent of this problem with respect to QLFD is unknown. Duplicate microcosm oral biofilms were grown from salivary inoculum on nitrocellulose filter-membranes laid onto blood agar. After seven days incubation under anaerobic conditions, the biofilms were extricated and examined by a custommade in vitro QLFD system delivering an irradiance of $750 \mu \mathrm{mol}$ $\mathrm{m}^{-2} \cdot \mathrm{s}^{-1}$. A measureable loss of plaque fluorescence $(\Delta \mathrm{F})$ due to photobleaching was evident in both microcosm biofilm samples with the highest rates of photobleaching observed immediately upon exposure to light. $\Delta \mathrm{F}$ readings were averaged, within discrete periods of time, to yield $\Delta \mathrm{F} \mathrm{min}^{-1}$ (arbitrary units normalised to 100 at time zero); 15.2 ( 0 to $1 \mathrm{~min}$ ), 6.79 (1 to $2 \mathrm{~min}$ ), 4.87 ( 2 to $5 \mathrm{~min}$ ), 2.17 ( 5 to $10 \mathrm{~min}$ ) and 1.29 (10 to $20 \mathrm{~min}$ ). Care must be taken when undertaking quantitative measurements of dental plaque fluorescence during longitudinal experiments and clinical investigations to avoid photobleaching.

Funding: University of Liverpool. 


\section{1 \\ Visualisation of Oral Biofilms Using Quantitative Light-Induced Fluorescence}

K. Bakht ${ }^{\text {a }}{ }^{*}$, C.K. Hope ${ }^{\text {a }}$, E. de Josselin de Jong ${ }^{\text {a, b }}$, S.M. Higham ${ }^{\text {a }}$, G.C. Martin ', G. Burnett ${ }^{\mathrm{c}}$

c.hope@liverpool.ac.uk

aUniversity of Liverpool, UK; ${ }^{b}$ Inspektor Research Systems BV,

Amsterdam, The Netherlands; 'GlaxoSmithKline, Weybridge, UK

Quantitative light-induced fluorescence (QLF) is a useful diagnostic tool for the detection and quantification of enamel caries in vivo, in vitro and in situ. Samples visualised under QLF, in general, display either red or green fluorescence. With particular pertinence to this study, red fluorescence has been shown to be associated with the presence of porphyrins, a by-product of bacterial metabolism and monitoring this type of fluorescence could provide insight into the microbial dynamics of plaque accumulation. This study aimed to correlate the QLF imaging of independent oral biofilms produced using an in vitro model with microbiological analysis as the model biofilm matured in order to evaluate the suitability of QLF as a diagnostic for plaque bacterial numbers. Independent oral biofilms representative of human plaque were produced using a constant-depth film fermenter (CDFF) operated under normal growth conditions. These biofilms were imaged using the QLFTMInVitro setup (Inspektor Research Systems BV, The Netherlands) and were analysed for fluorescence using ImageJ software $(n=16)$. Bacterial composition was also enumerated using traditional microbiological culture techniques. Results indicate that there is a weak negative correlation $(\mathrm{r}=-0.534, \mathrm{p}<0.05)$ between total microbial counts and intensity of red fluorescence (RF). Negative correlation $(r=-0.702$, $\mathrm{p}<0.05$ ) was also present between mutans streptococci and RF. Weak positive correlations were found between numbers of lactobacilli and RF $(r=0.466)$ and total streptococci and RF $(r=0.086)$ but these were not significant at the $5 \%$ level. Further work is required to corroborate the trends found here. This technique has the potential to improve our understanding of plaque biofilms and could be useful in evaluating therapies used to reduce plaque and other caries control strategies.

Supported by BBSRC GSK CASE.

\section{2 \\ Effect of Maltitol or Xylitol Sugar-Free Chewing Gums on Plaque Parameters Related to Dental Caries Development}

C. Thabuis ${ }^{\mathrm{a}}{ }^{*}$, V. Macioce $^{\mathrm{a}}$, B. Zhong ${ }^{\mathrm{b}}, \mathrm{S}$. Li ${ }^{\mathrm{b}}$, C. Lefranc-Millot ${ }^{\mathrm{a}}$, M. Berard ${ }^{c}, D$. Wils ${ }^{\text {a }, ~ L . ~ G u e r i n-D e r e m a u x ~}{ }^{a}$

clementine.thabuis@roquette.com

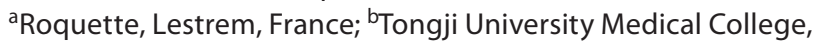

Shanghai, and 'Roquette, Shanghai, China

In order to better understand the role of polyols in dental caries prevention, the effects of maltitol and xylitol chewing gums (CG) on plaque parameters were assessed in a double-blind, par- allel, randomized, double-controlled study conducted in China. 240 children aged 13-15 years were divided into four groups: three groups chewing for 10 min either SweetPearl ${ }^{\mathrm{TM}}$-maltitol CG, XYLISORB ${ }^{\circledR}$-xylitol CG or gum base, 5 times/day for 30 days, and a control group without CG supplementation. Frequency of dental hygiene was defined, and plaque parameters (growth, $\mathrm{pH}$, bacteria, insoluble glucans) were evaluated at different times. The area under the curve of plaque $\mathrm{pH}$ was greater with polyols $\mathrm{CG}$ than with gum base (ANOVA, $\mathrm{p} \leq 0.05$ ) after 1 or 30 days of CG supplementation. Insoluble glucans level significantly decreased over study period in all gum groups $(-0.15 \mathrm{mg} / \mathrm{ml}$ for maltitol, -0.06 $\mathrm{mg} / \mathrm{ml}$ for gum base), and this reduction was more important with polyols than with gum base (Kruskal Wallis, $\mathrm{p}=0.02$ ). Plaque growth was reduced with maltitol CG ( -1.34 over experimental period) in a higher extent (Kruskal Wallis, $p=0.02$ ) than with gum base (-0.93). In comparison with gum base, polyols also lead to significant decreases in the concentrations of 5 bacteria species (Streptococcus mutans, Streptococcus sobrinus, Actinomyces viscosus, identified by plate method, and Porphyromonas gingivalis and Streptococcus pneumoniae, identified by real-time PCR) (ANOVA, $\mathrm{p} \leq 0.05$ ). Lastly, compared to the no-gum group, gum base induced a significant increase in plaque $\mathrm{pH}$ and significant decreases in insoluble glucans, plaque growth and bacteria. Therefore, this study showed that maltitol and xylitol CG induced similar modifications of plaque parameters, reflecting conditions favourable to dental caries prevention. In addition, polyols CG exhibited stronger effects than gum base CG, revealing a polyol effect on caries development.

Supported by Roquette.

\section{3 \\ Proteomic Analysis of Extracellular Matrix of Plaque-Like Biofilm Formed under Sucrose or Glucose and Fructose \\ G.P.Moi ${ }^{\mathrm{a}}{ }^{\mathrm{b}}$, * , J.A. Cury ${ }^{\mathrm{a}}$, A.F. Paes Leme ${ }^{\mathrm{b}}$ \\ adriana.paesleme@cebime.org.br \\ apiracicaba Dental School, UNICAMP, ${ }^{\text {b}}$ Center for Structural \\ Molecular Biology, ABTLuS, SP, Brazil}

Sucrose promotes changes in the protein composition of extracellular matrix (ECM) of plaque-like biofilm (PLB) and undetectable levels of calcium-binding proteins by two-dimensional gel electrophoresis (2-DE) in PLB formed in its presence provides further insight into the unique cariogenic properties of this dietary carbohydrate. To evaluate whether these changes are sucrose- dependent or the presence of its monosaccharides could also interfere with the protein profile an in situ study was conducted. During 2 phases of 14 days (in triplicates) a volunteer wore a palatal appliance containing 16 enamel blocks, being 8 in each side for PLB accumulation. In each phase, $20 \%$ sucrose solution or $\mathrm{H}_{2} \mathrm{Odd}$ and $10 \%$ glucose $+10 \%$ fructose solution or $\mathrm{H}_{2} \mathrm{Odd}$ were extraorally dripped onto each set of 8 blocks 8 times/day. On the 14th day, PLB were collected, ECM proteins were extracted and analyzed by two different approaches: (1) 2-DE followed by liquid chromatography coupled with mass spectrometry in tandem (LC-MSMS) and (2) whole extract proteins were analyzed by 
LC-MSMS. The results revealed differentially expressed proteins in $2-\mathrm{DE}$ were prolactin-induced proteins and calcium-binding proteins. Interestingly, the last proteins were found in 2-DE only in the $\mathrm{H}_{2} \mathrm{Odd}$ and glucose+fructose treatments. On the other hand, both proteins were found in the complex mixture analysis in $\mathrm{H}_{E} \mathrm{Odd}$, glucose+fructose and sucrose treatments. The calcium-binding proteins were found only in the sucrose whole extract and not in 2-DE, but in lower levels compared to $\mathrm{H}_{2} \mathrm{Odd}$ whole extract. These data suggest that the changes in the protein profile are not exclusively attributed to PLB formed in the presence sucrose and that the presence of calcium-binding proteins in glucose+fructose treatment could also modify the cariogenic potential of the PLB.

Supported by the Brazilian Funding Agency-Agency CAPES.

\section{4 \\ In situ Fluoride Binding to a Test Plaque after Fluoride Rinses \\ L.M.A. Tenuta *, A.A. Del Bel Cury, C.D.P. Kawachi, W.J. Silva, J.A. Cury \\ litenuta@fop.unicamp.br \\ Piracicaba Dental School, UNICAMP, Piracicaba, Brazil}

Fluoride $(\mathrm{F})$ can be taken up by biological (bacterial calcium (Ca)) and inorganic (calcium fluoride) reservoirs in dental plaque, which could contribute to maintain increased $\mathrm{F}$ concentrations in plaque fluid. In order to study $\mathrm{F}$ binding to bacterial $\mathrm{Ca}$ after a short-term exposure to $\mathrm{F}$, a test plaque was exposed in situ to $\mathrm{NaF}$ rinses at $\mathrm{F}$ concentrations of 0 (control), 52.6, 263.2 or $526.3 \mu \mathrm{M}$, all undersatured concerning calcium fluoride precipitation. In a double-bind, crossover study, a S. mutans IB1600 test plaque was mounted on palatal appliances, used by 10 volunteers for $15 \mathrm{~min}$ (enough time to allow Ca plaque binding from saliva according to a pilot study). Then, volunteers gently rinsed with the test solutions for $1 \mathrm{~min}$. After $30 \mathrm{~min}$, the test plaque and saliva were collected. Plaque fluid was separated from the bacterial solids; F and $\mathrm{Ca}$ concentrations in both and in saliva were determined. No significant difference in $\mathrm{Ca}$ concentrations in test plaque or saliva was found among the experimental groups $(p>0.05)$. In saliva, $F$ concentrations were below $2 \mu \mathrm{M}$, but significantly higher $(\mathrm{p}<$ 0.05 ) for groups 263.2 and $526.3 \mu \mathrm{M} \mathrm{F}$ when compared to the control. F concentrations in plaque fluid and solids increased linearly $(\mathrm{p}<0.05)$ with the increase in $\mathrm{F}$ concentration in the rinses, respectively (mean \pm SD; fluid: $7.1 \pm 2.0,8.4 \pm 2.5,12.3 \pm 2.9$ and $21.3 \pm 8.2 \mu \mathrm{M}$; solids: $27.5 \pm 14.0,42.4 \pm 14.2,46.6 \pm 13.0$ and $58.9 \pm 13.9 \mathrm{nmol} / \mathrm{g}$ wet weight). The results suggest that short-term exposure of plaque to low $\mathrm{F}$ concentrations is able to dose-dependently increase plaque biological F reservoirs, which could act as a source of $\mathrm{F}$ to plaque fluid.

Supported by CNPq (480212/2007-4) and FAPESP (2009/ 11464-2).

\section{5 \\ Fluoride Measurement of Plaque and Plaque Fluid from an in situ Model}

E.A. Martinez-Mier*, C. Buckley, P. Chandrappa, S.A. Kelly, D.T. Zero esmartin@iupui.edu

Indiana University School of Dentistry, Indianapolis, USA

Fluoride levels in plaque and plaque fluid are directly related to its anticaries effects. Therefore, models that incorporate these parameters while testing the anticaries potential of products would be valuable. The current project aimed at modifying an in situ model to measure plaque and plaque fluid fluoride with sufficient sensitivity and repeatability. 22 previously consented subjects participated in a two-treatment, examiner-blind study. A modification of the Koulourides model with partially demineralized enamel specimens covered with gauze was used. Two days before each leg, subjects received a dental cleaning. Under supervision, subjects brushed for one minute using 0 or $1,100 \mu \mathrm{g} \mathrm{F} / \mathrm{mg}$ F toothpaste. Then, subjects brushed twice daily for two weeks with their assigned toothpaste for one timed minute. At their next visit, plaque samples were obtained from the gauze. Several modifications to the collection and analysis techniques were tested. Plaque and plaque fluid fluoride were analyzed using modifications to the microdiffusion method and directly using a microelectrode. Plaque samples analyzed directly were acidified prior to analysis. Average $( \pm S D)$ plaque fluid fluoride was $0.25 \pm 0.12$ $\mu \mathrm{g} \mathrm{F} / \mathrm{mg}$ for the $0 \mu \mathrm{g} \mathrm{F} / \mathrm{mg}$ group, and $2.50 \pm 0.82$ for the 1,110 $\mu \mathrm{g}$ F/mg group. Average $( \pm \mathrm{SD})$ plaque fluoride was $2.03 \pm 1.57$ and $16.24 \pm 14.28 \mu \mathrm{g} \mathrm{F} / \mathrm{mg}$, for the $0 \mu \mathrm{g} \mathrm{F} / \mathrm{mg}$ group, measured directly and by diffusion; it was $41.23 \pm 55.60$ and $252.97 \pm$ $254.72 \mu \mathrm{g} \mathrm{F} / \mathrm{mg}$ for the $1,110 \mu \mathrm{g} / \mathrm{mg}$ group. All differences between the 0 and $1,100 \mu \mathrm{g} \mathrm{F} / \mathrm{mg}$ groups were statistically significant $(\mathrm{p}<0.01)$. ICCs for repeated analysis ranged from 0.97 to 0.99 . It is concluded that the technique rendered repeatable values when analyzing samples of unknown concentration and differentiated between groups exposed to different fluoride concentrations. 


\section{Caries Research}

\section{Session 6 Clinical Studies II}

\section{6 \\ Indirect Pulp Treatment in Primary Teeth after Long-Term Function \\ F.B. Araujo a, * L. Casagrande b ${ }^{\text {, R. Franzon }}{ }^{\text {a }}$, L.W. Bento
,
D.M. Dalpian a , F. García-Godoy \\ fernando.araujo@ufrgs.br \\ aDepartment of Pediatric Dentistry, Faculty of Dentistry, Federal University of Rio Grande do Sul, Porto Alegre, bSchool of Dentistry, Franciscan University Center (UNIFRA), Santa Maria, Brazil; 'Bioscience Research Center, College of Dental Medicine, Nova Southeastern University, Fort Lauderdale, Fla., USA}

Indirect pulp treatment (IPT) is a procedure where caries which is closest to pulp tissue is left in place and covered with a biocompatible material. The main goal of this minimally invasive technique is to maintain pulp vitality, thus avoiding a pulp exposure, while preserving dental substrate. To evaluate the clinical and radiographic outcomes of IPT in primary molars after longterm function, forty teeth were selected from 21 healthy children (4 to 8 years old). Teeth with deep carious lesions without signs and symptoms of irreversible pulpitis were randomized and divided into two groups, according to the capping material used over the demineralized dentin: Experimental group (1): $n=19$, self-etching adhesive system (Clearfill SE Bond); and Control group (2): $n=21$, calcium hydroxide liner (Dycal). Both groups were filled with composite resin $(3 \mathrm{M}-\mathrm{Z} 250)$ and submitted to a clinical and radiographic monitoring period until exfoliation. After the follow-up period (up to 60 months, 48 months mean time), 15 children were re-examined at a final evaluation. Six patients dropped out of the study and thirty-two teeth (baseline $=40$ ) were evaluated. Twenty-five cases met the criteria for clinical and radiographic success, reaching an overall success rate of $78 \%$ with no statistical difference between the groups $(p=678)$. Failures occurred after the first year of follow-up, and were detected by radiographic evaluation. Second molars and the occlusal restorations presented higher frequency of failures, when compared to first molars and occluso-proximal restorations, but it was not statistically significant. These results showed that IPT has a high clinical and radiographic performance in primary teeth after long-term function and is not dependent on the capping material used over the carious dentin.

\author{
107 \\ Chemical Analysis of Carious Dentine after \\ Incomplete Dentine Caries Removal and Sealing \\ E. Oliveira ${ }^{\text {a }}$ M. Bueno ${ }^{\text {a }}$, C.S. Bavaresco ${ }^{\text {b, }}{ }^{*}$, M. Maltz ${ }^{\text {b }}$ \\ mmaltz@ufrgs.br \\ aFaculty of Odontology, Pelotas Federal University, and \\ bFaculty of Odontology, Federal University of Rio Grande do Sul, \\ Brazil
}

This study evaluated the effect of calcium hydroxide cement (CHC) and glass ionomer cement (GIC) on the calcium, phosphate and fluoride dentine content after incomplete dentine caries removal (IDCR) and sealing. Forty-one permanent teeth with deep lesions were submitted to IDCR, application of CHC, GIC or wax (placebo) and sealing for 3 months. Following the IDCR and the sealing period (SP), dentine sample was collected from the pulpal wall of the cavity. Calcium, phosphorus and fluoride dentine content was measured (biochemical) after (IDCR) and after treatment (SP). Differences among the groups were tested by Kruskal-Wallis test and the effect of the treatment in each group by Wilcoxon test. Data is presented as percentile (25-50-75). One case of pulp necrosis was observed (wax). An increased phosphorus concentration was observed in dentine collected after treatment in the CHC $(0.16-0.225-0.40$; $\mathrm{p}<0.05)$ and wax $(0.07-0.12-$ $0.39 ; \mathrm{p}<0.05)$, while in the GIC the increased was not significant (0.0875-0.22-0.41; $\mathrm{p}>0.05)$. Calcium levels were higher after SP with CHC $(0.13-0.36-0.63 ; \mathrm{p}<0.05)$ while in the GIC $(0.24-$ $0.49-0.60 ; \mathrm{p}>0.05)$ and wax (0.21-0.28-0.51; $\mathrm{p}>0.05)$ only a tendency was observed. No differences in calcium and phosphorus content were observed among the groups after IDCR and SP $(p>0.05)$. No difference in the calcium and phosphorus ratio was observed among the groups after the IDCR and SP $(p<0.2)$. Fluoride content increased after treatment with GIC (1,389.45$3,472.75-14,685.02$; $\mathrm{p}>0.05)$ but not in CHC (128.25-365.15579.25 ; $p>0.05)$ and wax (157.4-250.6-430.95; p > 0.05). Differences in fluoride content was observed among the groups ( $\mathrm{p}>$ $0.05)$. The results indicate an increase in mineral content in the sealed carious dentine, a suggesting tissue remineralization.

Supported by FAPERGS. 


\section{8 \\ Photodynamic Therapy for Disinfection of Infected Dentine: A Phase I Clinical Trial}

J.P.F. Longo ${ }^{\text {a, }}{ }^{*}$, S.C. Leal ${ }^{\text {b }}$, A.R. Siminoni ${ }^{c}$, A.C. Tedesco $^{c}$, R.B. de Azevedo ${ }^{a}$

jplongo82@gmail.com

aDepartment of Genetics and Morphology, Institute of Biology, University of Brasília, 'b Department of Dentistry, Universidade de Brasília, 'Department of Chemical and Philosophy, University of São Paulo, Ribeirão Preto, Brazil

The aim of this study was to evaluate the efficacy of Photodynamic Therapy (PDT) mediated by Aluminum-chloro-phthalocyanine $(\mathrm{AlClFt})$ in reducing cariogenic bacteria in carious lesions. 10 subjects presenting class I carious lesions involving $2 / 3$ of dentine, determined by radiograph analysis in primary/permanent molars, without pulp and periodontal disease were enrolled in the study. The lesions were treated according to the Atraumatic Restorative Treatment (ART), except for the application of PDT in part of the infected dentine before restoration, as follows: the infected dentine was totally removed from the mesial half of the lesion and chips were collected for bacteria analysis, while the distal half was removed and chips collected after being treated with PDT. PDT was performed in 3 steps: 1 . application of liposomal AlClFt photosentizer in the cavity for $5 \mathrm{~min}$; 2 . removal of the excess photosentizer with water; 3. cavity irradiation with red laser with $180 \mathrm{~J} / \mathrm{cm}^{2}$. PDT efficacy was assessed by quantifying the cariogenic bacteria in dentine chips collected before (mesial) and after (distal) the PDT treatment. These dentine chips were immediately placed into a $1 \mathrm{ml}$ eppendorf containing BHI (Brain Hearth Infusion) Broth and transported to the laboratory within $60 \mathrm{~min}$. The eppendorfs were shacked for $1 \mathrm{~min}$ after which the supernatants were collected (500 $\mu \mathrm{l})$. Supernatants samples were analyzed for the total number of cariogenic bacteria after the colony forming units (CFU) counting in BHI agar-broth. The t-test was used to compare the number of CFUs in dentine before and after the application of PDT. The results showed a reduction of $82 \%$ of total cariogenic bacteria after PDT application $(\mathrm{p}<0.0001)$. This clinical trial I demonstrated the disinfection capacity of the PDT protocol mediated by AlClFt.

Supported by Conselho Nacional de Desenvolvimento Científico e Tecnológico (CNPq - Brasil).

\section{9 \\ Human Dental Pulp Vasculogenesis Evaluated by CD34 Antigen Expression}

A. Sotirovska-Ivkovska ${ }^{\text {a, }}{ }^{*}$, E. Zabokova $^{\text {a }}$, L. Popovska ${ }^{\text {b }}$, L. Ivkovski ${ }^{\mathrm{c}}$

anasotirovska@yahoo.com

Departments of a Pedodontic Dentistry, and ${ }^{\mathrm{b} C}$ Cariology and Endodontology, School of Dentistry, 'Department of Histopathology and Clinical Cytology, Institute for Radiotherapy and Oncology, School of Medicine, Skopje, Macedonia

Vascular development involves vasculogenesis, in which endothelial cells form a tubular network. Development and maintenance of the vascular system requires not only the formation of new vessels, but also the continuous adjustment of vessels structures in response to functional needs. CD34 is an endothelial marker that is extensively used in dental pulp for the identification of pulpal vasculature. This study investigated the responses of the immune system under 3 different clinical conditions: healthy teeth, shallow and deep cavities. Teeth were extracted and immediately cut longitudinally, pulp tissue was extirpated and fixed in formalin for $24 \mathrm{~h}$ at $4^{\circ} \mathrm{C}$. The specimens were embedded in paraffin, according to standardized laboratory procedure. Sections were cut at $5 \mu \mathrm{m}$ thicknesses and stained by the streptavidin-biotin complex immunoperoxidase method. Dental pulps were obtained from permanent teeth, morphologically examined at light microscope level and with immunohistochemistry. To characterize the vascularization of human dental pulp, we examined the expression of the human haematopoietic progenitor cell antigen CD34. The findings indicate that vasculogenesis of dental pulp is process that is present in healthy teeth with single CD34 positive cell. In carious teeth these cells subsequently coalesce to form solid vascular cords inside the connective tissue, which later aggregate with the progression of the carious lesion. Pericites were embedded within the newly formed microvessels basement membrane. The presence of CD34 endothelial cells reveals the continuous adjustment of vessels in response to functional needs and dental tissue homeostasis. Endothelial cells play a key role in immune and inflammatory reactions by regulating lymphocyte and leukocyte movement into dental pulp.

Supported by The Ministry of Education - Republic of Macedonia.

\section{0 \\ Qualitative and Quantitative Analysis of Tertiary Rats \\ L. Nemeth * \\ lidija.nemeth@mf.uni-lj.si \\ Medical Faculty, University of Ljubljana, Slovenia} Dentin after Cavity Preparation and Acid Etching in

The aim of this study was to qualitatively and quantitatively analyse tertiary dentin after three restorative procedures in different time intervals post-operatively, comparing the response in young and old rats. The study was conducted on $48 \mathrm{Wistar}$ rats (45 days and 18 months old). On the intact first upper molar in each quadrant three different restorative procedures were made: (A) cavity preparation with phosphoric acid etching and a filling; (B) cavity preparation only with a filling; (C) cavity preparation, etching with one step self-etching adhesive and a filling. Young and old animals were divided into four groups: 3, 7, 30 and 60 days post-operatively. On histologic specimens, stained with haematoxylin and eosin, the appearance of tertiary dentin was investigated and its thickness measured. In young teeth there was a faster onset of tertiary dentin synthesis; after etching with phosphoric acid its thickness was the greatest and its structure more regular than in old teeth. 30 days post-operatively in young animals the tertiary dentin thickness was greater after procedures $\mathrm{A}$ and $\mathrm{B}(\mathrm{p}<0.029$ and $\mathrm{p}<0.023)$ in comparison with procedure $\mathrm{C}$. In old animals there were no differences in tertiary dentin thickness between the three procedures. 30 and 60 days post-operative- 
ly after etching with phosphoric acid the thickness of tertiary dentin was greater in young animals $(\mathrm{p}<0.031$ and $\mathrm{p}<0.015)$ compared to old animals. We concluded that in old teeth phosphoric acid etching has less stimulative effect on tertiary dentin synthesis than in young teeth.

Protocol of study approved by the Veterinary Ethic Committee of the Republic Slovenia (323-02-798/2005).

\section{1 \\ Use of Carisolv as a Chemical Pre-Treatment to Reduce Fissure Sealant Microleakage - An in vitro Study}

A.B. Schenkel *, V.P. Thompson

abs5@nyu.edu

New York University College of Dentistry, New York, USA

Aim: Resin-based sealant placed in the occlusal pits and fissures of posterior teeth is accepted as an effective means of preventing dental caries. Improvements in the technique could lead to even greater effectiveness. This study investigated a method of improving the sealing procedure using Carisolv as a chemical pretreatment for enhancing bonding effectiveness of a resin-based pit and fissure sealant. Methods: 16 decay free molar teeth were selected from teeth extracted at New York University College of Dentistry. The occlusal surface of each tooth was cleaned using a water-slurry of coarse pumice in a standard dental prophy cup. Six teeth had Carisolv applied for $60 \mathrm{~s}$, rinsed, dried, etched with $37 \%$ phosphoric acid, rinsed, dried, and had sealant applied according to the manufacturer's instructions (Ultraseal XT Plus, Ultradent Corp, Salt Lake City). The other ten teeth were etched and sealed as above with no chemical pre-treatment after prophy. Each sample was subjected to 50,000 mouth-motion occlusal $50 \mathrm{~N}$ load cycles to simulate time in the mouth under function. All samples were then exposed to caries indicator dye (Sable Seek, Ultradent Corp) for $24 \mathrm{~h}$, rinsed, sectioned and examined with light microscopy. Microleakage was measured as a percentage representing the length of dye penetration along the total length of the sealant-tooth interface. Results: Samples pre-treated with Carisolv showed an average of $29 \%(S D=27)$ microleakage, as opposed to an average of $59 \%(\mathrm{SD}=10)$ microleakage for samples with no pre-treatment. This difference was found to be statistically significant at $p=0.05$. Conclusions: Pre-treatment of the fissure with Carisolv before etching may add to the effectiveness of the adhesion of the resin sealant by possibly removing the pellicle and organic remnants in the fissure system not reached by prophy.
112

\section{Early Occlusal Caries Management Supported by Quantitative Light-Induced Fluorescence}

M.R. Alammari, P.W. Smith *, E. de Josselin de Jong, S.M. Higham

P.W.Smith@liverpool.ac.uk

School of Dental Sciences, University of Liverpool, UK

An increased proportion of the total caries burden is found in fissures and subsequent restorative repair is costly in terms of time, resources and oral health. Caries diagnosis is important not only in preventive caries strategies but also in minimising and guiding operative interventions. The objective of this study was to determine whether the QLF parameters $\Delta \mathrm{F}$ and $\Delta \mathrm{Q}$ were appropriate for aiding diagnosis and clinical decision making of early occlusal caries by comparing QLF analysis with actual restorative management. Following ethical approval 46 subjects attending a dental teaching hospital were enrolled into the study. White light digital (WL) and QLF images/analyses of 46 unrestored posterior teeth with suspected occlusal caries were made after a clinical decision had already been taken to explore the fissure operatively. WL and QLF imaging/analysis were repeated after initial cavity preparation, and the type of restorative treatment was determined by the supervising clinician independent of any imaging performed. The actual restorative management carried out was recorded as a fissure sealant/preventive resin restoration (FS) or a class I occlusal restoration (Rest.) thus reflecting the extent of operative intervention. All QLF images obtained were analysed independently without knowing the treatment outcome. The results showed statistically significant differences between the two treatment groups $\Delta \mathrm{F}(\mathrm{p}=0.002)($ mean $\pm \mathrm{SD}: 22.60 \pm 5.60-\mathrm{FS}$ and $28.80 \pm 6.06$-Rest.) and $\Delta \mathrm{Q}(\mathrm{p}=0.012)$ (mean $\pm \mathrm{SD}: 230.49$ \pm 161.82 -FS and $348.30 \pm 235.94$-Rest.) thus higher values of $\Delta \mathrm{F}$ and $\Delta \mathrm{Q}$ were associated with more extensive restoration. $\Delta \mathrm{F}$ and $\Delta \mathrm{Q}$ values may be useful in aiding clinical diagnosis and supporting decision making in relation to the restorative management of occlusal caries.

Funding: International scholarship from King Abdulaziz University, Kingdom of Saudi Arabia.

\section{3 \\ The Use of Computer-Assisted Light Fluorescence for the Assessment of Caries Excavation}

M.J. Altenburger *, P. Ganter, E. Hellwig, K.-T. Wrbas, A. Al-Ahmad

markus.altenburger@uniklinik-freiburg.de

Department of Operative Dentistry and Periodontology,

University Hospital and Dental School Freiburg, Germany

The aim of caries excavation is to remove bacterially contaminated dentine but to take care of sound dentine areas. Current methods to evaluate the completeness or to detect areas of incomplete caries excavation, e.g. the visual-tactile or the dye staining method, are dissatisfying. The aim of the present in vitro study was to assess the usefulness of a computer-assisted light fluorescence device (VistaProof) for the control of caries excavation. 50 
teeth with cavitated carious lesions were excavated with a rosehead drill and intermittently analyzed with the VistaProof-system. Excavation was stopped when no more fluorescence could be observed. Then the cavities were checked with the visual-tactile or the staining method (Caries Detector), respectively. In each 25 additional lesions, complete caries removal was first checked with the staining or the visual-tactile method. Subsequently the cavities were analysed with the VistaProof-system. From all cavity floors dentine samples were gathered and evaluated according to bacterial DNA. Additionally, every tooth was sectioned and microhardness (KHN) was tested in $50 \mu \mathrm{m}$ steps perpendicularly to the cavity floor. All cavities, where conventional methods were used to check for complete caries excavation were free of bacterial DNA, while in $6 \%$ of the cavities examined with VistaProof DNA could be detected. However $50 \%$ of the cavities checked first with VistaProof could have been further excavated when examined with conventional methods. Cavities tested with VistaProof were significantly softer (up to $300 \mu \mathrm{m}$ distance from the cavity floor) compared to those examined with the conventional methods. All methods indicated the completeness of caries removal sufficiently. However, only VistaProof was able to classify according to infected and affected (but not infected) dentine and might therefore be a useful tool to avoid overexcavation.

Supported by Duerr Dental AG, Bietigheim-Bissingen, Germany.

\section{4 \\ Discomfort of Different Methods for Approximal Caries Detection in Primary Molars}

F.M. Mendes *, T.F. Novaes, R. Matos, J.C.P. Imparato, D.P. Raggio, M.M. Braga

fmmendes@usp.br

School of Dentistry, University of Sao Paulo, Brazil

This in vivo study aimed to evaluate the discomfort reported by children after utilization of different methods for approximal caries detection in primary molars. Seventy-six children (age 4-12 year-old) had approximal surfaces examined by visual inspection (ICDAS), radiographic examination, laser fluorescence device (DIAGNOdent pen, LFpen), and direct visual examination after temporary separation with orthodontic rubber. For discomfort assessment, we used Wong-Baker faces scale which is a six-point scale from 0 (a smiling face, indicating no discomfort) to 5 (a crying and sad face, indicating great discomfort). After each diagnostic method, examiner asked the child to indicate the face which represented his/her feeling regarding the method. For temporary separation with orthodontic rubber method, the discomfort assessement was carried out immediately after placing the rubber and in the recall visit, seven days after. Friedman's test was used to compare the discomfort degree among the methods. Poisson regression analysis was used to evaluate association between gender, age and type of dentition with the discomfort degree. Temporary separation method was performed in only 50 children. Radiographic and LFpen methods presented similar discomfort degree (mean $\pm \mathrm{SD}=0.72 \pm 1.13$; and $0.76 \pm 1.02$, respectively) and higher than ICDAS $(0.42 \pm 0.72)$. Considering the temporary sep- aration immediately after placing the rubber, the discomfort was higher than using ICDAS $(0.84 \pm 1.02)$ and similar to other methods. After seven days, however, there was no difference compared to other methods $(0.64 \pm 0.96)$. Older children (Rate ratio, $95 \%$ $\mathrm{CI}=0.73,0.57-0.94)$ and children with mixed dentition $(0.32$, $0.17-0.62)$ reported less discomfort with the LFpen. Nevertheless, for temporary separation, higher discomfort was reported by older children (1.19, 1.04-1.36). In conclusion, radiographic, temporary separation and LFpen methods provoke higher discomfort than ICDAS, but generally the scores of discomfort are low.

Supported by Conselho Nacional de Desenvolvimento Cientifico e Tecnologico - CNPq - Brazil (Process No. 476372/2006-2, 565061/2008-9, 302368/2008-6 and 471952/2008-7).

\section{5 \\ Comparative Analysis of Two Different Colorimetric Buffering Test Kits}

\section{B. Buchgraber *, A. Lintner, P. Städtler}

barbara.buchgraber@medunigraz.at

Department of Dentistry and Maxillofacial Surgery, Division of Preventive and Operative Dentistry, Endodontics, Pedodontics, and Minimally Invasive Dentistry, Medical University Graz, Austria

The most commonly used caries risk diagnosis kits are the colorimetric method for detecting individual buffering capacity. Although the paper strip method is easy to use, the colour matching method with colour guides is maybe problematic. We therefore determined the colour agreement between five different operators (raters) using two different colorimetric methods. Stimulated saliva samples were gathered from 35 subjects. Saliva buffering capacity was assessed, Saliva check with three test pads A, B, C and CRT-buffer with one test pad. One drop of saliva was dispensed onto each test pad and after 2 to $5 \mathrm{~min}$ photos were taken, and shown immediately to five different raters who had to examine 70 photos. The colours of the test pads were compared with the buffer colour chart defining the buffering capacity. With Saliva check colour matching for pad A was 77\%, for pad B 71\% and for pad C 79\% while for CRT-buffer colour matching between the five operators was $78 \%$. The buffering capacity agreement between the two kits was $22.7 \%$. To assess the amount of agreement among raters different statistical measures were considered: Fleiss' kappa, Leight's weighted kappa, Krippendorff's alpha and Kendall's concordance coefficient and iota, which is used to analyze the amount of agreement among the raters using both tests. These statistical tests showed that with Saliva check there was no significant agreement among the raters $(p=0.957)$ whereas with CRT-buffer there was only weak concordance $(\mathrm{p}<0.004)$ and the iota value $(0.0542)$ indicated there was no significant agreement between the test kits. The coefficients considered here account not only for the percentage agreement but also for the percentage of agreement expected by chance. If the coincidence is not considered the results may misrepresent the overall agreement. The percentage agreement suggested there was a good colour matching between the five raters but more sophisticated analysis found that this was not statistically significant and so the clinical relevance of such buffering tests must be questioned. 


\section{6 \\ Clinical Performance of DIAGNOdent in Occlusal Caries Detection}

W. Dukic ${ }^{\mathrm{a}, *}$, S. Kadic ${ }^{\mathrm{b}}$, V. Picek ${ }^{\mathrm{b}}$, B. Delija $^{\mathrm{a}}$, O. Lulic $^{\mathrm{a}}$

dukic@sfzg.hr

aSchool of Dental Medicine, University of Zagreb, and

${ }^{\mathrm{b}}$ Dental Polyclynic Zagreb, Croatia

The aim of this study is to clinically analyse efficacy of detection of fissure caries with laser fluorescence method (DIAGNOdent) on different locations of the fissure system. Occlusal surfaces of 123 permanent molars were analysed with DIAGNOdent device on five different locations in the fissure system; mesial, central, distal, oral and buccal fissure. A total of 579 different fissures of the occlusal surfaces were examined with DIAGNOdent device according to caries score: $0-20$ no caries, 21-99 caries. The fissures were opened (PFO) with Komet Micropreparation set No. 4377 burs and analysed with the following caries scores: 0 - no caries, 1 - enamel caries, 2 - dentin caries. From the total of 579 analysed fissures, $323(55.8 \%)$ did not have any caries, $166(28.7 \%)$ had enamel caries and $90(15.5 \%)$ had dentine caries. The specificity (SP) and sensitivity (SE) of DIAGNOdent for different fissures is as follows: mesial 0.93 (SP) 0.71 (SE), central 0.85 (SP) 0.92 (SE), distal 0.92 (SP) 0.96 (SE), buccal 0.83 (SE) 0.92 (SE) and oral 0.88 (SE) 0.97 (SP). The overall SP and SE for DIAGNOdent was 0.88 and 0.92 . Positive predictive value of DIAGNOdent device in different fissures is as follows: distal $96.3 \%$, central $88.7 \%$, oral $84.3 \%$, mesial $78.5 \%$ and finally buccal $66.7 \%$, the latter being statistically different from other fissures (chi square test, d.f. $=4$, $\mathrm{p}<0,001)$. According to PFO, enamel caries occurs most frequently in distal fissure (43.9\%) and dentine caries in central fissure $(23.5 \%)$ and that is statistically significant (chi square, d.f. $=$ $8, \mathrm{p}=0.001$ ). DIAGNOdent device showed a high degree of accuracy in analysis of occlusal fissure system which enables the precise analysis of different parts of that system and therapy by the principles of minimally invasive preparation.

\section{7 \\ Reliability of the Nyvad Caries Diagnostic and Lesions Activity Assessment Criteria}

\section{M.C. Séllos *, V.M.Soviero}

marianasellos@yahoo.com.br

Rio de Janeiro State University, Rio de Janeiro, Brazil

This study aimed to evaluate the reproducibility of Nyvad's caries diagnostic criteria in the primary teeth and to verify the average examination time using this method. Eighty children (3-7 years old) participated in the study. Parents signed an informed consent and the study was approved by the Committee for Ethics in Research. Examinations were performed after supervised toothbrushing in the dental chair under standardized conditions, artificial light and compressed air by two calibrated examiners trained by the criteria's authors. Examination time was measured using a digital chronometer. Inter- and intra-examiner reliability was expressed as percentage agreement (\%) and kappa
( $k$ ) at tooth surface level, using the following cut-off points: (1) sound $\times$ diseased; (2) active $\times$ inactive; (3) discontinuity level $\times$ sound; (4) cavity level $\times$ sound. Inter-examiner results were: (1) $k=0.82$; (2) $k=0.80$; (3) $k=0.90$; (4) $k=0.95$. Intra-examiner results were: (1) $k=0.86$; (2) $k=0.86$; (3) $k=0.94$; (4) $k=0.98$. The majority of disagreements were concentrated between sound and non-cavitated surfaces $(65.3 \%$ - 158/242): $33.5 \%$ (81/242) between sound and non-cavitated inactive, $26.0 \%$ (63/242), sound and non-cavitated active and 5.8\% (14/242), non-cavitated active and inactive. The mean examination time was $226.5 \mathrm{~s}$ ( \pm 128.53$)$. It was concluded that Nyvad's caries diagnostic criteria showed high reliability and suitable examination time, being consistent and reliable for dental caries clinical studies in primary teeth.

\section{8 \\ Treatment Decision for Deep Caries Lesions in Public Health Service in Porto Alegre, Brazil \\ C.M. Weber, L.S. Alves, M. Maltz * \\ mmaltz@ufrgs.br \\ Faculty of Odontology, Federal University of Rio Grande do Sul, Brazil}

Several treatments have been proposed for deep carious lesions, focusing mainly the traditional complete caries removal and stepwise excavation. Considering the evidences that complete caries removal in deep caries lesions increases the risk of pulp exposure, the aim of this study was to assess the impact of treatment decisions in deep caries lesions on Public Health Service in the city of Porto Alegre, Rio Grande do Sul, Brazil. A structured enquery and three simulated clinical cases were presented per dentist. Fifty-four out of 122 professionals of the service, participated in the study. There was no observed difference between respondents and non-respondents regarding gender, year of graduation and specialty (chi-square test, $\mathrm{p}>0.05$ ). In a total of 155 observations, the most indicated procedure was the complete caries removal in a single session (61.30\%), followed by complete caries removal in two sessions (11.61\%), incomplete caries removal in two sessions (18.06\%) and incomplete caries removal in a single session $(9.03 \%)$ (chi-square test, $\mathrm{p}<0.05)$. There was no association between treatment and gender, university of graduation and specialty (chi-square test, $\mathrm{p}>0.05$ ). Although, there was significant association between year of graduation and proposed treatment. Dentists graduated after 1990 proposed the incomplete caries removal more often than dentists graduated in previous years (chi-square test, $\mathrm{p}=0.04$ ). The prevalent proposed treatment is the one with higher rate risk of pulp exposure and bad prognosis. Younger dentists tend to indicate more conservative approaches. The wide variation in restorative practices shows the importance of continuous dental education programs.

Supported by CAPES, CNPq. 


\section{9}

\section{Caries Management Decisions in France}

S. Doméjean *, S. Tubert-Jeannin

sophie.orliaguet@u-clermont1.fr

CHU Clermont-Ferrand, Service d'Odontologie, Hôtel-Dieu,

Clermont-Ferrand, France

Aims: To explore the caries management decisions in private practice in France through (1) a questionnaire survey and (2) a practice-based survey. Methods: (1) A French translation of a questionnaire used by Espelid et al. [Espelid and Tveit: Acta Odontol Scand 2001;59:285-289; Espelid et al.: Acta Odontol Scand 2001;59:21-27] in Scandinavia, was sent to a random sample ( $n=2003)$ of French general private practitioners. (2) Another sample of French practitioners $(n=100)$ was asked to record the characteristics of 35 preventive or restorative treatments made on vital permanent teeth. Results: (1) Responses were received from 830 dentists. (2) Twenty-six practitioners recorded the characteristics of 921 treatments performed on 457 patients. Results: (1) Answers indicated a tendency towards early restorative intervention in a young adult with low caries activity and good oral hygiene. They also showed large variations between the treatment decisions of French dentists. (2) Results indicated that participants rarely performed non-invasive treatments. Of all therapies, $66 \%$ were initial treatments (IT) (85\% were restorations, $15 \%$ noninvasive treatments). The respondents used an inappropriate detection tool, as most of treatment decisions were based on visual inspection frequently associated with probing. Multidimensional analyses allowed a better understanding of the factors influencing the decision making: it showed that dentists provided different restorative treatments depending on patient characteristics, with minimally invasive, esthetic restorations preferentially performed for healthy, young and well-insured patients. Restorative treatments and detection tools also varied markedly among practitioners. These variations in service patterns were not related to a specific patient profile in each dental practice. Conclusions: Recent concepts in caries management have not yet been adopted in everyday practice. Pathophysiology seemed not to be the only factor influencing the decision-making in caries management.

Funding: Survey 2 was funded by the Clermont-Ferrand regional university hospital, France, through a Projet Hospitalier de Recherche Clinique (hospital-led clinical research project).

\section{0 \\ Incidence of Caries around Restorations on a Longitudinal Study Using ICDAS}

A.G. Ferreira Zandona ${ }^{\mathrm{a},}{ }^{*}$, E. Santiago $^{\text {b }}{ }^{\text {, G.J. Eckert }}{ }^{\mathrm{c}}$, D.T. Zero $^{\mathrm{a}}$

azandona@iupui.edu

andiana University School of Dentistry, Indianapolis, buniversity of Puerto Rico School of Dentistry, San Juan, Indiana University School of Medicine, Indianapolis, USA

Caries around restorations and sealants (CARS) are the major reason for restoration replacement and represent diagnostic challenges. International Caries Detection and Assessment System (ICDAS) has been increasingly accepted, but there is only limited information regarding its use for longitudinal CARS assessment. This study aims to evaluate the prevalence and incidence of CARS in a 32-month interval in 569 children enrolled in rural schools in the Commonwealth of Puerto Rico who followed longitudinally using ICDAS. Associations with CARS prevalence and incidence were performed using GEE methods applied to logistic regression. Out of 2,953 restored surfaces examined at baseline, 298 surfaces (10\%) had lesions ICDAS score $\geq 1$ and 121 surfaces (4\%) had ICDAS score $\geq 3$. During the 32 -month follow-up $12 \%$ of the surfaces developed a new lesion ICDAS score $\geq 1$ and $6 \%$ ICDAS score $\geq 3$. At baseline and 32 months $96 \%$ of the surfaces were restored with amalgam restorations, $2 \%$ with tooth-colored restorations and the remainder with provisional restorations and crowns. There was a significant difference $(p<0.01)$ between the restoration type and the prevalence of CARS, with temporary fillings having a higher prevalence followed by tooth-colored restorations. Primary teeth had significantly higher incidence of CARS than permanent teeth $(\mathrm{p}<0.001)$. There were significant differences between surfaces $(p=0.0001)$ for both prevalence and incidence, buccal surfaces having a higher prevalence and incidence when lesions ICDAS score $\geq 1$ were included and occlusal surfaces having a higher prevalence and incidence for lesions ICDAS score $\geq 3$. Following placement of a new restoration, 111 of 1,080 surfaces (10\%) developed a lesion ICDAS score $\geq 1$ around the restoration, with 92 lesions occurring within 1 year of placement and 21 having ICDAS score $\geq 3$. In conclusion, ICDAS allows detailed analysis of CARS prevalence and incidence.

Supported by NIH/NIDCR RO1DE017890-01.

\section{1 \\ Comparison of Isolation Methods for Sealing Teeth in a Mobile Program}

\author{
A.E. Soto-Rojas *, K.M. Yoder, C. Krushinsky, C. Eberhardt, \\ G. Maupome-Carvantes \\ arsoto@iupui.edu \\ Indiana University School of Dentistry, Indianapolis, USA
}

Clinical success of dental sealants may be influenced by placement technique, isolation and surface preparation. The aim of this study was to compare the survival of dental sealants placed using 2 different isolation techniques. Seal Indiana is a statewide mobile dental program that provides preventive oral health services including dental sealants, oral examinations, and fluoride varnish applications. Dental students have provided these services under faculty supervision since 2003. Up until 2006, isolation technique performed included use of cotton rolls, dry angles, and high volume suction. From 2007 to date, the Isolite System (Santa Barbara, Calif., USA) has been mostly used to provide isolation. Preparation of teeth prior to sealant placement (surfaces cleaned with dry toothbrush and etched for $15 \mathrm{~s}$ with $60 \%$ phosphoric acid), the sealing criteria (sealing sound surfaces and non-cavitated incipient caries), and the sealant brand (Delton, Dentsply, York, Pa., USA) have remained identical since 2003. Records from children seen at least a second time by Seal Indiana were assessed for dental sealants survival. Survival was calculated based on records of the need to replace a sealant. Comparisons for survival of dental sealants placed using the two isolation methods were 
done using Cochran-Mantel-Haenszel tests. 660 children 6 to 18 years old were evaluated at least twice during 2003-2006; survival rate was $34 \%$ ( 481 sealants lost $/ 770$ sealants placed). 834 children were evaluated at least twice during 2007-2009; survival rate was 39\% (518 sealants lost/940 sealants placed). Differences were not statistically significant. Isolation approaches did not accrue significant differences for dental sealants clinical success, as determined by survival rates in a non-randomized assessment undertaken in an educational, service/learning, outreach seal mobile program.

Supported by Department of Preventive and Community Dentistry, Indiana University School of Dentistry.

\section{2 \\ Influence of Oral Hygiene Knowledge and Habits on Dental Fear in Croatian Preschool Children}

H. Juric * Z. Matosic, A. Novacic

juric@sfzg.hr

School of Dental Medicine, University of Zagreb, Croatia

Preschool age is defined as the time from age 3 to age 6 . This period in a child's life is when important attitudes and oral hygiene habits are developed, and dental fear can be a severely limiting factor in dental health maintenance. The aim of the present study was to assess oral hygiene habits and the quality of dental fear in Croatian preschool children, and to try to define statistically significant differences, with respect to age, sex and geographical background. The research was conducted by a questionnaire method on 796 preschool children (391 boys and 405 girls), aged $3-6$ years (mean $=4.96$ years), in two big cities located in different geographical areas (Split - coast or south area, and Zagreb - continental or north area of Croatia). Statistical data processing was conducted by implementing the $\chi^{2}$ test. Results show that there is a difference in oral hygiene habits between children in Zagreb and Split. Children in Zagreb had better oral hygiene habits $(\mathrm{p}=0.001)$. Different age and gender also had an influence on the results; the older the children the less the parents participated in maintaining their children's oral hygiene $(\mathrm{p}<$ 0.001 ), while girls had better oral hygiene habits. On daily basis $89.7 \%$ of girls and $81.5 \%$ of boys brush their teeth. This study also demonstrated that children are mostly afraid of the dental drill (50.9\% of children with dental fear). Furthermore, dental fear is not necessarily related to previous experiences. This research has shown that the level of oral hygiene and oral hygiene habits in preschool children in Croatia is unsatisfactory. This might be due to a low dental health awareness in parents and their children and a collapse of adequate funding of the primary preventive health care system in Croatia.

\section{3}

\section{Evaluation of Clinical Parameters Associated with Caries Activity in Primary Teeth}

\author{
M.M. Braga ${ }^{\text {a, }}$, R. Matos ${ }^{\text {a }}$, T.F. Novaes ${ }^{\text {a , J.C.P. Imparato }}{ }^{\text {a }}$, \\ K.R. Ekstrand ${ }^{\mathrm{b}}$, F.M. Mendes ${ }^{\mathrm{a}}$ \\ mmbraga@usp.br

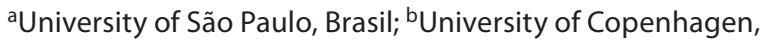 \\ Denmark
}

Several clinical parameters are, intentionally or not, evaluated in activity assessment of caries lesion. Caries recording systems, the Nyvad criteria (NY) and an adjunct to the ICDAS system (LLA-ICDAS), have considered these parameters differently during the process of caries activity assessment. The aim of this in vivo study was to evaluate the association between clinical parameters and the decision concerning lesion caries activity in primary teeth, using NY and LAA-ICDAS. Agreement between both indices was also used. Occlusal surfaces of 757 primary molars in 139 children (3-12 years old) were classified as sound, having inactive or having active caries lesions using separately each system. Clinical parameters, as roughness, potential for plaque stagnation, surface integrity and staining, were also recorded in carious sites $(n=347)$. Association among these parameters and presence of active caries on occlusal surfaces were evaluated using univariate and multiple logistic analysis and adjusted Odds ratio (OR; $95 \% \mathrm{CI}$ ) was calculated for all models. In univarite analysis, rough sites were more prone to be active lesions (NY-OR: 15.7;9.0-27.3; LAA-ICDAS-OR: 29.0; 9.7-86.4, p $<0.001)$, as well as whitish or yellowish sites when compared to dark-brownish sites (NY-OR: 10.0; 5.5-18.1; LAA-ICDAS-OR: 18.8; 8.2-43.4, p < 0.001). Cavitated lesions were more frequently active than noncavitated lesions (NY-OR: 2.4; 1.4-4.0; LAA-OR: 8.2; 2.4-27.6, p < 0.001). Otherwise, plaque stagnation potential were only associated with activity by LAA-ICDAS (NY-OR: 2.2; 0.95.01, p = 0.07; LAA-OR: $7.4 ; 2.9-19.0)$. In the multiple models, all these variables were retained, but showing different power of association. Considering agreement between systems, explanatory models pointed possible differences between system activity assessment. Therefore, clinical parameters, as roughness, staining, potential for plaque stagnation and surface integrity, can be used solely in caries lesions activity assessment, since they presented significant association, but their association is stronger when they are used jointly.

Funding: Conselho Nacional de Desenvolvimento Científico e Tecnológico (CNPq), Brasil - 476372/2006-2 and 565061/2008-9.

\section{4 \\ Activity Assessment of Caries Adjacent to Amalgam Fillings Using Freshly Extracted Permanent Teeth \\ K.R. Ekstrand ${ }^{\mathrm{a}, *}$, G. Douglas $\mathrm{G}^{\text {b }}$, J. Wang ${ }^{\text {c }}$, D.N.J. Ricketts ${ }^{\mathrm{c}}$ \\ kim@odont.ku.dk \\ aUniversity of Copenhagen, Denmark; beeds Dental Institute, \\ 'School of Dentistry, Dundee, UK}

This study had two main aims: (1) to devise and determine the reproducibility of a visual-tactile scoring system to assess the activity of lesions adjacent to amalgam restorations and (2) to test the 
accuracy of the devised system. Based on the literature the following 3 visual-tactile predictors were devised to assess lesion activity adjacent to teeth restored by amalgam: (A) Visual appearance of the tooth next to the restoration; (B) Tactile feeling of the tooth next to the restoration (ball ended probe, gently drawn over surface) and (C) location of the lesion next to the restoration. Each of the 3 predictors $(\mathrm{A}, \mathrm{B}, \mathrm{C})$ had sub scores which were given points from 0 to 3 , where lower scores indicated inactivity and higher scores indicated lesion activity. The cumulative score or predictive value could be between 0 and 7. A threshold was established, thus a cumulative score of $\geq 4$ predicted the lesion as being active and below arrested or sound. The accuracy of the scoring system was tested on 90 sites on 64 freshly extracted teeth with amalgam restorations. The validation was performed by using methyl red - a dye method recognized for being red when $\mathrm{pH}$ is $<5.5$ and yellow when $\mathrm{pH}$ is $>5.5$. Intra-examiner-reproducibility of the 3 variables was found to be substantial to excellent. The combined sum of specificity and sensitivity for the scoring system was 1.80 . Thus, it is possible fairly accurately to assess the activity of lesions adjacent to amalgam restorations by means of the devised scoring system.

\section{5 \\ Fluoride Modification of Bleaching Gel to Reduce Erosion and Erosion/Bleaching Associated Enamel Softening \\ F.J. Wegehaupt ${ }^{\mathrm{a}, *}$, H. Witzke ${ }^{\mathrm{b}}$, T. $_{\text {. }}$ ttin $^{\mathrm{a}}$ \\ florian.wegehaupt@zzmk.uzh.ch \\ ${ }^{a}$ Clinic for Preventive Dentistry, Periodontology and Cariology, University of Zurich, Switzerland; 'bentist private practice, \\ Lengede, Germany}

This in vitro study aimed to determine if the admixture of fluoride compounds to bleaching gel reduces the erosion and erosion/bleaching associated loss of enamel microhardness. Moreover the effect of fluoride modification of bleaching gel on the uptake of fluoride should be assessed. 200 bovine enamel samples were randomly allocated to ten groups: A1 and A2 (no gel, control), B1 and B2 (fluoride and carbamide peroxide free gel), $\mathrm{C} 1$ and C2 (10\% carbamide peroxide gel), D1 and D2 (10\% carbamide peroxide gel with $0.5 \%$ amine fluoride) and E1 and E2 (10\% carbamide peroxide gel with $0.5 \%$ sodium fluoride). In samples of groups A1-E1 the amount of $\mathrm{KOH}$-soluble and structurally bound fluoride was determined after performing a 10-day bleaching cycling with $2 \mathrm{~h}$ application of the respective substance at $37^{\circ} \mathrm{C}$ and humid conditions and $22 \mathrm{~h}$ storage in artificial saliva. Microhardness of groups A2-E2 was determined at baseline and after two times performing the following erosion/bleaching cycle: $2 \mathrm{~h}$ treatment with the respective substance at $37^{\circ} \mathrm{C}$ and humid conditions, $1 \mathrm{~h}$ storage in artificial saliva, $15 \mathrm{~s}$ erosion with $1 \%$ citric acid and $1 \mathrm{~h}$ storage in artificial saliva. Data were statistically analysed using ANOVA and Scheffe post-hoc tests. Groups B1 and C1 showed a significantly lower amount of $\mathrm{KOH}$-soluble and structurally bound fluoride compared to group A1 while the amount of both fluoride kinds was significantly increased in groups D1 and E1. Highest loss of microhardness $(\Delta \mathrm{KHN})$ could be observed in groups B2 and C2 while loss of microhardness was significant- ly lower for groups D2 and E2 compared to B2 and C2. Bleaching associated loss of $\mathrm{KOH}$-soluble and structurally bound fluoride could be avoided by fluoride modification of the bleaching gel furthermore the microhardness loss due to erosion/bleaching is reduced by this modification.

\section{6 \\ Appearance of Natural Enamel after Topical Fluoride Treatments and Abrasion in vitro}

\author{
K.R. Stenhagen ${ }^{\text {a, }}{ }^{*}$, A.B. Tveit ${ }^{\text {a }}$, L.H. Hove ${ }^{\text {a }}$, K. Kvam ${ }^{\text {b }}$ \\ k.r.stenhagen@odont.uio.no \\ aFaculty of Dentistry, University of Oslo, Norway; \\ ${ }^{\text {b} N o r d i c ~ I n s t i t u t e ~ o f ~ D e n t a l ~ M a t e r i a l s, ~ H a s l u m, ~ N o r w a y ~}$
}

$\mathrm{TiF}_{4}$ and $\mathrm{SnF}_{2}$ solutions are known to produce a surface coating on enamel. The aim of this pilot study was to investigate the enamel surface after topical treatment with these solutions, $\mathrm{NaF}$ and Bifluorid 10 and the effect of brushing (abrasion). Natural human enamel surfaces were treated with $\mathrm{TiF}_{4} 0.5 \mathrm{M} \mathrm{pH} 1.2, \mathrm{SnF}_{2}$ $0.5 \mathrm{M} \mathrm{pH} 1.2$, NaF $0.5 \mathrm{M} \mathrm{pH} 8.0$ solutions for $2 \mathrm{~min}$ and Bifluorid 10 for $20 \mathrm{~s}$ (manufacturers application instruction) respectively, air dried and analysed in a scanning electron microscope (SEM). Additionally enamel surfaces treated in the same way were abraded for $2 \mathrm{~min}$ in a standardized tooth brushing machine prior to the SEM analysis. A total of 24 specimen surfaces were investigated. The $\mathrm{TiF}_{4}$ treatment resulted in a nearly continuous surface coating with small cracks and precipitates on top. The precipitates were removed by the abrasion, but the coating remained intact except for a few abrasion grooves. Images following $\mathrm{SnF}_{2}$ treatment showed an incomplete thin coating which nearly disappeared after abrasion. Analyses after $\mathrm{NaF}$ treatment revealed no alterations of the surface compared with the controls. The Bifluorid 10 treatment resulted in a coating with precipitates. Abrasion resulted in distinct grooves, but a thin coating covered by a considerable amount of $\mathrm{CaF}_{2}$-like globules remained on the surface. In conclusion, the coatings formed after $\mathrm{TiF}_{4}$ and Bifluorid 10 treatment under the present experimental procedure, were abrasion resistant in contrast to the thin coating formed following $\mathrm{SnF}_{2}$ application. The enamel surfaces treated with $\mathrm{NaF}$ had the same appearance as the control surfaces both before and after abrasion.

Funding: University of Oslo. 


\section{Caries Research}

\section{Session 7 \\ Diagnostics}

\section{7 \\ In vivo Reproducibility of ICDAS II for Pit and Fissure Caries Detection \\ D.F. Côrtes ${ }^{\mathrm{a}, *}$, V.L.M. Soviero ${ }^{\mathrm{b}}$, R.J. Arkader ${ }^{\mathrm{b}}$, R.P. Ellwood ${ }^{\mathrm{c}}$ \\ denise.cortes@uol.com.br \\ aFederal University of Juiz de Fora, 'b State University of Rio de \\ Janeiro, Brazil; ' Dental Health Unit, Manchester University, UK}

The aim of this in vivo study was to evaluate the reproducibility of ICDAS II for caries detection. The permanent molars, premolars and upper incisors of 72 subjects ( $8-18$ years) were examined. A total of 1,560 pit and fissure surfaces were evaluated by one trained examiner using the ICDAS II primary caries detection criteria. The ICDAS codes ' 0 ' and ' 2 ' were split and additional codes for 'stain' ( $0 \mathrm{~A})$ and 'brown caries discoloration' (2A) were included. The examination was repeated after one week for evaluation of the intra-examiner reproducibility. The unweighted kappa found was 0.76 for the traditional ICDAS II and it has increased to 0.81 when the 'stain' and 'brown caries discoloration' were considered as one additional code. The weighted kappa was 0.91 for both ICDAS criteria. The proportion of agreement per code was higher than $90 \%$ for codes ' 0 sound' and ' 6 extensive dentin cavity'. The lowest agreement (32\%) was found for code ' 1 first enamel change'. The agreement has ranged from $51 \%$ to $63 \%$ for traditional codes ' 2 ', ' 3 ', ' 4 ' and ' 5 ' and for codes ' $0 \mathrm{~A}$ and ' $2 \mathrm{~A}$ '. The proportion of agreement has increased to $75 \%$ when 'stain' and 'brown caries discoloration' were considered as one joined code. The poorest agreement per code was found for the white caries discoloration visible after drying. Sound and extensive dentin cavity codes showed the highest agreement. The substantial reproducibility for ICDAS II in vivo has slightly increased when stain and brown caries discoloration were considered as an additional code, as well the proportion of agreement for that joined code.

Supported by Colgate Palmolive Co, UK.

\author{
128 \\ How Reliable Are Members of the ICDAS Core Group \\ at Using the ICDAS? An in vitro Study

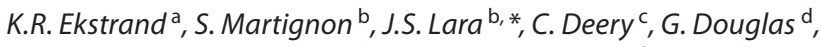 \\ H. Eggertsson ${ }^{\text {e }, ~ L . F . ~ G a m b o a ~}{ }^{\mathrm{b}}$, A. Ferreira-Zandona ${ }^{\mathrm{f}}$, J. Kolker ${ }^{\mathrm{g}}$,

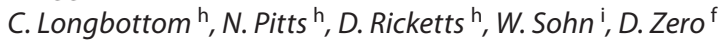 \\ kim@odont.ku.dk \\ aUniversity of Copenhagen, Denmark; ' Universidad El Bosque, \\ Bogotá, Colombia; 'University of Sheffield, 'University of Leeds, \\ UK; ' Indianapolis, Ind., 'Indiana University School of Dentistry, \\ Indianapolis, 9University of lowa, lowa City, USA; hUniversity of \\ Dundee, UK; 'University of Michigan, Ann Arbor, USA
}

The aim of this study was to investigate the intra-examiner and inter-examiner reproducibility of the ICDAS-system when used by eleven members from the ICDAS Core-Group. The material comprised 64 extracted teeth selected from a bank of teeth stored in thymolised water at $7^{\circ} \mathrm{C}$. Prior to the examinations the teeth were cleaned with a toothbrush and water. Photographs taken of predetermined sites on the tooth surface were printed in black and white, simply to direct the examiners where to score. The eleven examiners discussed the seven ICDAS scores in a $1 \mathrm{~h}$ session prior to the examinations, although as members of the ICDAS-Core Group all examiners had extensive experience of the System. The teeth were placed in random order along a table with one meter between each position, together with the photograph indicating the site to score. There were $30 \mathrm{~s}$ available to score each tooth, which were placed in water to avoid dehydration between examinations. At each position there was a three-in-one syringe and a ball-ended probe. The teeth were re-scored the following day, but the tooth sequence was altered. The intra-examiner and inter-examiner reproducibility is expressed by means of weighted kappa (WK) and percentage agreement (PA). Inter-examiner reliabilities among all examiners ranged from WK of 0.69-0.92 and PA 87.9-96.8\%. The WK-median was 0.82 . The intra-examiner reliability of the 11 examiners varied between WK $0.84-0.98$ and PA 93.1-99.3\% and the WK-median was 0.9. Conclusion: Under in-vitro conditions the ICDAS-Core Group can produce good levels of agreement when using the ICDAS system.

Universidad El Bosque, Bogotá, Colombia has partially funded this study. 


\section{9 \\ The Impact of Clinical Experience and Magnification on Reproducability of ICDAS II for Occlusal Caries}

P. Mitropoulos *, C. Rahiotis, A. Kakaboura, G. Vougiouklakis

pgmitrop@dent.uoa.gr

Faculty of Dentistry, National and Kapodistrian University of

Athens, Greece

This in vitro study investigated the impact of clinical experience and magnification on degree of agreement with implementation of the ICDAS II criteria. The occlusal surfaces of 38 extracted non-cavitated intact teeth received classification according to the ICDAS II criteria separately by 3 post-graduate students having received only theoretical training (Group A) and 3 post-graduate dental students having 1-year clinical experience in the ICDAS II criteria (Group B). Two different dentists with 1-year clinical experience in the ICDAS II criteria examined separately the same teeth in two sessions, with a 7-day interval. The procedure was repeated after 2 months with use of magnification loupes $(\times 2.8)$. The weighted kappa values (mean $\pm \mathrm{SE}$ ) for group A and B were: $0.85 \pm 0.07 / 0.83 \pm 0.08$ (grade 0$), 0.65 \pm 0.07 / 0.73 \pm 0.06$ (grade 1), $0.75 \pm 0.12 / 0.8 \pm 0.1$ (grade 2$)$ and $0.94 \pm 0.12 / 1 \pm 0$ (grade $3)$, respectively. The intra-examiner kappa weighted values for examiner 1 for examination without and with magnification were $0.89 \pm 0.12 / 0.86 \pm 0.05$ (grade 0$), 0.83 \pm 0.07 / 0.71 \pm 0.11$ (grade 1), $0.82 \pm 0.17 / 0.82 \pm 0.15$ (grade 2), $0.89 \pm 0.1 / 0.96 \pm 0.1$ (grade $3)$, respectively. For examiner 2 the corresponding values were: $0.98 \pm 0.07 / 0.93 \pm 0.04$ (grade 0$), 0.84 \pm 0.12 / 0.73 \pm 0.15$ (grade 1), $0.89 \pm 0.16 / 0.84 \pm 0.1$ (grade 2 ) and $0.99 \pm 0.08 / 0.98 \pm 0.01$ (grade 3 ). The inter-examiner kappa weighted values without and with magnification were $0.83 \pm 0.07 / 0.83 \pm 0.13$ (grade 0$), 0.77$ $\pm 0.12 / 0.67 \pm 0.14($ grade 1$), 0.87 \pm 0.11 / 0.81 \pm 0.08$ (grade 2$)$ $0.91 \pm 0.1 / 0.91 \pm 0.16$, respectively. In conclusion, grade 0 and grade 3 ICDAS scores are highly reproducible irrespectively of clinical experience. Additionally, clinical experience in the ICDAS II criteria improves the degree of interexaminer agreement for grade 2 , but not for grade 1 . The examination under magnification loupes does not improve inter-examiner and intraexaminer agreement relatively to visual examination.

\section{0 \\ ICDAS-II and DIAGNOdent Reproducibility for Detection of Occlusal Caries by Undergraduate Students and Experienced Dentists}

M.A. Stacey *, M.S. Hopcraft, D.L. Bailey, M.A. Abuzar, G.G. Adams, N.J. Cochrane

mastacey@unimelb.edu.au

Melbourne Dental School, The University of Melbourne,

Australia

The aim of this study was to compare the reproducibility of the ICDAS-II visual-based system for occlusal caries detection with the reproducibility of the DIAGNOdent laser fluorescence-based system for occlusal caries detection when used by undergraduate dental students and experienced dentists. Six undergraduate dental students and three experienced dentists were trained in the use of ICDAS-II using a commercially available computer program, and in the use of the DIAGNOdent by means of the manufacturer's instructions. The students and dentists then assessed the occlusal surfaces of 120 permanent molars twice, with a 2 -week interval between the first and second assessments, using the ICDAS-II, and later repeated this cycle using the DIAGNOdent. DIAGNOdent readings were classified as described by Lussi et al. [J Dent 34:467-471]. Intra-examiner Cohen's kappa values for ICDAS-II ranged from $0.56-0.66$ for students and $0.63-0.78$ for dentists. Intra-examiner kappa values for DIAGNOdent ranged from 0.49-0.58 for students $0.51-0.60$ for dentists. Each student and dentist scored a higher intra-examiner kappa value for ICDAS-II than for DIAGNOdent. Inter-examiner Cohen's kappa for ICDAS-II ranged from $0.37-0.62$ for students and $0.47-0.58$ for dentists. Inter-examiner kappa values for DIAGNOdent ranged from $0.29-0.66$ for students and $0.49-0.62$ for dentists. The dentists had similar inter-examiner kappa scores for both techniques. Agreement between students was variable with some achieving higher agreement with ICDAS-II and others with DIAGNOdent. Despite the fact there was no formal calibration process ICDAS-II had greater intra-examiner reproducibility for occlusal caries detection compared with the DIAGNOdent for both students and dentists.

Funding: Melbourne Dental School.

\section{1 \\ The Outcome of a Training Session in the ICDAS-System - A Reliability Study among Dentists in Kuwait \\ Y. Altarakemah ${ }^{\mathrm{a}, *}$, M. Alsane a , K. Ekstrand ${ }^{\mathrm{b}}$, A. Ismail ${ }^{\mathrm{c}}$, N. Pitts ${ }^{\mathrm{d}}$, D. Ricketts ${ }^{\text {d }}$ E.S. Akpata ${ }^{a}$ \\ yaltarakemah@yahoo.com \\ aKuwait University, Kuwait; bUniversity of Copenhagen, Denmark; 'Maurice H. Kornberg School of Dentistry, Temple University, USA; dUniversity of Dundee, UK}

Four members of the ICDAS-core group were invited November 2009 by the Faculty of Dentistry at Kuwait University to give 1 day of lectures, followed by a 2 day hands-on training on the International Caries Detection and Assessment System (ICDAS). The aim of this study was to report the reliability outcomes achieved during the hands on training, thus at the early parts and at the end of the training sessions. Ten dentists participated in the training, which lasted for 4 sessions. In the first session the dentists worked on extracted teeth with lesions representing all 7 ICDAS codes. The second and third sessions involved examining selected teeth in 12 patients $(n=45$ teeth, Surfaces $=220)$, and 13 patients $(n=49$ teeth, Surfaces $=245)$ respectively. All teeth were examined under acceptable clinical conditions. The first and second session was followed up by discussions with the ICDAS-core group members. The inter-examiner agreement using weighted Kappa after session 2 varied in the 45 tests between $0.57-0.81$ with the arithmetic mean of 0.71 and the median on 0.72 . Correspond- 
ing figures after the third session were $0.83-0.93 ; 0.87$; and 0.87 . A paired t-test showed a significant improvement in performance from the second to the third session; $\mathrm{t}=-17.7 ;$ d.f. $=44 ; \mathrm{p}<0.0001$. In the fourth session, 9 of the 10 dentist's re-assessment at least $50 \%$ of the teeth examined at earlier sessions. The intra- examiner reproducibility also using weighted kappa values varied between $0.89-0.97$. The median was 0.91 . To conclude, it is possible in a short time to teach dentists the ICDAS system so they can achieve acceptable reliability values. No effort was done to measure the accuracy.

\section{2 \\ Effect of an E-Learning Programme on the Performance of ICDAS in Detecting Occlusal Caries}

J.A. Rodrigues *, I. Hug, A. Lussi

jorodrigues@hotmail.com

Department of Preventive, Restorative and Pediatric Dentistry, School of Dental Medicine, Bern, Switzerland

This study aimed to evaluate the effect of an e-learning programme on the performance of ICDAS scores in detecting occlusal caries. To do so, 97 human permanent molars were selected and one site in each occlusal surface was chosen as test site. Four dentists, who never had any contact with ICDAS before, scored the sites twice (before and after the e-learning programme available on http://icdas.smile-on.com) with a one-week interval according to the ICDAS codes. One expert examiner, who has a large experience with ICDAS, also examined the sites once. The teeth were histologically prepared and assessed for caries extension. Weighted kappa (wK), sensitivity, specificity and area under the ROC curve (AUC) were calculated at $D_{1}, D_{2}$ and $D_{3,4}$ thresholds before and after the e-learning programme. McNemar test was performed to compare the values of sensitivity, specificity before and after the programme. The averages of wK values were 0.61 (before) and 0.66 (after) for interexaminer reproducibility. The sensitivity values before and after the programme were 0.80 and $0.79\left(\mathrm{D}_{1}\right), 0.71$ and $0.63\left(\mathrm{D}_{2}\right)$ and 0.80 and $0.70\left(\mathrm{D}_{3,4}\right)$, respectively. Specificity values were 0.66 and $0.66\left(D_{1}\right), 0.69$ and $0.77\left(D_{2}\right)$ and 0.83 and $0.89\left(\mathrm{D}_{3,4}\right)$. AUC values varied from 0.77 to 0.88 (before) and from 0.78 to 0.86 (after), with no statistical difference. McNemar test did not show any difference between the values before and after the programme. Correlation (wK) with histology was 0.62 (before), 0.63 (after) and 0.62 (expert examiner). It can be concluded that the e-learning programme did not have any statistical significant effect on the performance of ICDAS, although this visual score system performed well in detecting occlusal caries.

Funding: University of Bern.

\section{3 \\ Performance of Fluorescence-Based Methods, ICDAS-II and Radiographic Examination to Detect Occlusal Caries Lesions in Primary Teeth}

J.F. Souza ${ }^{\mathrm{a}, *}$, T. Boldieri $^{\mathrm{a}}$, M.B. Diniz ${ }^{\mathrm{a}}$, J.A. Rodrigues ${ }^{\mathrm{b}, \mathrm{c}}$, A. Lussi $^{\mathrm{b}}$, R.C.L. Cordeiro ${ }^{a}$

ritacord@foar.unesp.br

a Araraquara Dental School, UNESP, Universidade Estadual

Paulista, Brazil; 'b School of Dental Medicine, University of Bern,

Switzerland; ' Cruzeiro do Sul University, UNICSUL, São Paulo,

Brazil

The aim of this in vitro study was to evaluate the performance of fluorescence-based methods, radiographic examination and visual inspection for occlusal caries detection in primary teeth. Seventy-eight primary molars with occlusal surfaces varying from sound to to different stages of caries lesions were selected. After professional tooth cleaning, one site of each teeth was assessed twice by 2 examiners using the DIAGNOdent 2095 (LF), DIAGNOdent 2190 (LFpen), VistaProof (FC), ICDAS criteria for visual inspection and bitewing radiographs (BW). The teeth were histologically prepared and assessed for caries extension. Optimal cut-off limits for LF, LFpen and FC were established by the maximal sum of sensitivity and specififity. Considering threshold 1 , the sensitivities values were statistically similar each other for FC (0.83) and ICDAS (0.75) and LF (0.63) LFpen (0.71), who showed the best value for threshold 3 (1.00). The better specificities values were for BW (1.0) for threshold 1 and LF (0.92) ICDAS (0.94) and BW (0.94) for threshold 3. Spearman correlation coefficients with histology were 0.71 (LF), 0.65 (LFpen), 0.74 (FC), 0.71 (ICDAS) and 0.64 (BW). The area under the ROC curve exhibited values statistically similar varied from 0.803 to 0.945 . Inter- and intraexaminers intraclass correlation values were respectivety 0.86 and 0.92 (FC), 0.85 and 0.86 (LF), 0.84 and 0.88 (LFpen). ICDAS weighted Kappa values for inter- and intraexaminer were 0.83 and $0.88-0.96$, respectively; and BW Kappa values were 0.73 and $0.75(\mathrm{BW})$, respectively. In conclusion, the methods evaluated exhibited good reproducibility and good validity for occlusal caries detection in primary teeth.

Funding: FAPESP (2009/00218-0) and CAPES - Brazil.

\section{4 \\ In vitro Detection and Measurement of Dental Caries Using QLF Compared to ICDAS and Histological Assessment \\ K.R. Ekstrand ${ }^{\mathrm{a}}$, S. Martignon ${ }^{\mathrm{b}}$, A. Cortes ${ }^{\mathrm{b}}{ }^{\mathrm{*}}$, R.P. Ellwood ${ }^{\mathrm{c}}$ \\ kim@odont.ku.dk \\ aUniversity of Copenhagen, Denmark; ' Universidad El Bosque, \\ Bogotá, Colombia; ' University of Manchester, UK}

This in-vitro study aimed at comparing the detection and measurement of dental caries using QLF with the ICDAS system and histological assessment. In 58 cleaned permanent teeth a preselected site was scored (KE) using the ICDAS codes $0-6$ with a 
head-light, WHO-probe and three-in-one syringe. The teeth were also imaged using QLF (Inspektor Pro, The Netherlands) and later images were analyzed to calculate deltaQ representing 'lesion volume' with a mineral loss threshold of $5 \%$. A repeat assessment was conducted after 15 days. $220 \mu \mathrm{m}$-thick sections were histologically assessed with a stereomicroscope at the lesion/scored area center for demineralisation (KE) as follows: $0=$ no demineralisation; 1 = demineralisation limited to outer-enamel half; $2=$ demineralisation involving inner-enamel half to outer-dentine $1 / 3 ; 3=$ demineralisation involving middle-dentine $1 / 3$; and $4=$ demineralisation involving inner-dentine $1 / 3$. ICDAS scores were distributed among the surfaces as follows: $0=10 ; 1=5 ; 2=9 ; 3=$ $9 ; 4=7 ; 5=11$; and $6=7$. The intraclass correlation coefficient between the first and second QLF assessments was 0.79. For classification of disease based on histological thresholds of score: $>1$, $>2$ and $>3$, the area under the ROC's were 1.00, 0.96 and 0.90 and for the same thresholds, the maximum Sensitivity and Specificities were 100 (Se) and 100 (Sp), 100 (Se) and 88 (Sp) and $86(\mathrm{Se})$ and $83(\mathrm{Sp})$ respectively. For classification of disease based on ICDAS thresholds of $>1,>2$ and $>3$, the area under the ROC's were $0.99,0.98$ and 0.95 and for the same thresholds, the maximum Sensitivity and Specificities were 100 (Se) and 90 (Sp), 93 (Se) and $100(\mathrm{Sp})$ and $79(\mathrm{Se})$ and $96(\mathrm{Sp})$ respectively. In this in vitro study, the QLF system showed good reproducibility and was strongly associated with histological and ICDAS assessments of dental caries.

Universidad El Bosque, Bogotá, Colombia has partially funded this study.

\section{5 \\ In vitro Reproducibility and Accuracy of ICDAS and FOTI Examinations of Dental Caries with Histological Validation}

K.R. Ekstrand ${ }^{\text {a }}$, S. Martignon ${ }^{\text {b }}$, J. Gomez ${ }^{\text {b, * }}$, C. Longbottom ${ }^{\mathrm{c}}$, R.P. Ellwood ${ }^{\mathrm{d}}$

kim@odont.ku.dk

aUniversity of Copenhagen, Denmark; ${ }^{b}$ Universidad El Bosque, Bogotá, Colombia; 'School of Dentistry, Dundee, dUniversity of Manchester, UK

This study investigated the intra-examiner reproducibility and accuracy in detecting caries and estimating lesion depth of the ICDAS-system alone and also supplemented with Fibre Optic Trans-Illumination (FOTI). Fifty-eight permanent teeth surfaces were visually assessed (KE) with the ICDAS codes 0-6 using a head-light, WHO-probe and three-in-one syringe. Same surfaces were examined by two examiners (RE, CL) using the ICDAS criteria supplemented with FOTI (Microlux, AdDent, USA) and a consensus decision was recorded based on the following additional FOTI criteria: $0=$ no shadow/stained area; $1=$ thin-grey-shadow into enamel; 2 = wide-grey-shadow into enamel; 3 = microcavitation with wide-grey-shadow in enamel and no evidence of dentine shadow; $4=$ orange/brown or bluish/black shadow $<2$ $\mathrm{mm}$ wide; $5=$ orange/brown or bluish/black shadow $>2 \mathrm{~mm}$ wide and/or trans-illumination light-blocked; 6 = large frank-cavita- tion area with likely pulpal involvement; 9-thin stain preserving same width when transilluminated. A repeat examination of all teeth was conducted after 1 day. $220 \mu$ m-thick sections were histologically assessed with a stereomicroscope at the lesion/scored area center for demineralisation (KE) as follows: $0=$ no demineralisation; 1 = demineralisation limited to outer-enamel half; $2=$ demineralisation involving inner-enamel half to outer-dentine $1 / 3 ; 3=$ demineralisation involving middle-dentine $1 / 3$; and $4=$ demineralisation involving inner-dentine $1 / 3$. ICDAS scores were distributed among the surfaces as follows: $0=10 ; 1=5 ; 2=9 ; 3=$ $9 ; 4=7 ; 5=11$; and $6=7$. Intra-examiner agreement using weighted Kappa values for the ICDAS, FOTI and the histological assessments were $0.96 ; 0.88 ; 0.98$, respectively. The association between ICDAS and FOTI, ICDAS and Histology and FOTI and Histology, using a Spearman correlation coefficient were 0.95, 0.92 and 0.91 . The intraclass correlation coefficients for the same comparisons were $0.95,0.85$ and 0.84 respectively. Both ICDAS and the FOTIscoring system had excellent intra-examiner reproducibility and were strongly associated with histological scores under in vitro conditions.

Universidad El Bosque, Bogotá, Colombia has partially funded this study.

\section{6 \\ Thermal Images under Pulse Heating for Quantification of Sound, De- and Re-Mineralized Enamel in vitro \\ M. Ando a, * , T. Sakagami ${ }^{\text {b }}$, G.J. Eckert ${ }^{\text {c }}$, D.T. Zero ${ }^{\text {a }}$ \\ mando@iupui.edu \\ andiana University School of Dentistry, Department of Preventive and Community Dentistry, Indianapolis, USA; ${ }^{b}$ Kobe University Graduate School of Engineering, Department of Mechanical Engineering, Kobe, Japan; 'Indiana Univeristy School of Medicine, Division of Biostatistics, Indianapolis, USA}

This study evaluated whether pulse heating thermal images could distinguish sound, de- and re-mineralized enamel. 24 extracted adult lower incisors were covered with paraffin wax except on the labial surface of two $1 \times 1 \mathrm{~mm}^{2}$-windows. Specimens were divided into 2 groups, and were demineralized by exposure to Streptococcus mutans A32-2 in trypticase-soy-broth containing $5 \%$ sucrose at $37^{\circ} \mathrm{C}$ for 3 or 6 days. One window was covered. Specimens were further divided into 2 groups, and were remineralized with 250 or 1,100 ppm-F as NaF for 10 days by $\mathrm{pH}$-cyclicmodel [3 days-demineralization (3 days-demin)/remineralized with 250 ppm-F (250 ppm F-remin), 6 days-demineralization (6 day-demin)/250 ppm F-remin, 3 days-demin/remineralized with 1,100 ppm-F (1,100 ppm F-remin), and 6 days-demin/1,100 ppm F-remin]. The distribution of the infrared radiation energy was acquired by the infrared camera (100 images/s). A xenon flash lamp was the heat source (1,600 J and excitation-time: 1/1,200 s). Sound, demineralized, and remineralized enamel were analysed. The temperature descent curve between $\operatorname{Ir}$ (intensity of the infrared radiation energy) and $t$ (elapsed time after heating) was fitted to the equation with coefficients $A$ and $B: \operatorname{Ir}=-A \ln (t)+B$. Repeat- 
ed measures ANOVA models were used to compare the coefficient $A$ estimates between groups and treatment periods (sound, demin, remin). Average $\pm \mathrm{SD}$ of $A$ (sound, demin, remin) were: 3 days-demin/250 ppm F-remin: $342 \pm 38,347 \pm 60,413 \pm 81$, 6 days-demin/250 ppm F-remin: $363 \pm 69,522 \pm 63,572 \pm 46$, 3 days-demin/1,100 ppm F-remin: $348 \pm$ 51, $455 \pm$ 96, $447 \pm$ 109, and 6 days-demin/1,100 ppm F-remin: $351 \pm 39,539 \pm 89$, $501 \pm 57$. There was no difference for sound period among groups ( $p>0.05)$. For demin, 3 days-demin/250 ppm F-remin was lower than the other groups. For remin, 6 days-demin $/ 250$ ppm F-remin was higher than 3 days-demin/250 ppm F-remin and 3 days-de$\min / 1,100 \mathrm{ppm}$ F-remin $(\mathrm{p}<0.05)$. Remin was higher than sound for 3 days-demin/250 ppm F-remin $(p<0.05)$. Demin and remin were higher than sound for the other 3 groups $(p<0.05)$. These results showed that pulse heating thermal imaging has the potential for detection and quantification of non-cavitated enamel caries.

\section{7 \\ Evaluation of Different Methods for Occlusal Caries Detection: An in vivo Study with Histological Validation}

M.B. Diniz ${ }^{\mathrm{a}, *}{ }^{*}$, T. Boldieri $^{\mathrm{a}}$, L. Santos-Pinto ${ }^{\text {a }}$, J.A. Rodrigues ${ }^{\mathrm{b}, \mathrm{c}}$, A. Lussi $^{\text {b }}$, R.C.L. Cordeiro ${ }^{\text {a }}$

mibdiniz@hotmail.com

${ }^{a}$ Araraquara Dental School, UNESP, Universidade Estadual

Paulista, Araraquara, Brazil; bSchool of Dental Medicine, University of Bern, Switzerland; 'C Cruzeiro do Sul University, UNICSUL,

São Paulo, Brazil

The aim of this in vivo study was to evaluate the validity of different methods for occlusal caries detection in permanent teeth. This study was approved by the Ethics Committee in $\mathrm{Re}$ search of the Araraquara Dental School, UNESP (50/08). Eightyeight adults aged 18-35 years participated in the study; a total of 105 posterior permanent teeth with indication of extraction were selected. After professional tooth cleaning, one occlusal site per tooth varying from sound to different stages of caries was selected. One experienced examiner performed the examinations using: DIAGNOdent 2095 (LF), DIAGNOdent 2190 (LFpen), VistaProof (FC), ICDAS visual criteria and bitewing radiographs (BW). After extraction, the teeth were histologically prepared and assessed for caries extension. Optimal cut-off limits were established for LF, LFpen and FC. The detection methods were compared by means of sensitivity, specificity, accuracy and area under the ROC curve. The specificities at D1 and D3 were, respectively, 1.00 and 0.77 for LF, 0.80 and 0.71 for LFpen, 0.80 and 0.49 for FC, 0.60 and 0.77 for ICDAS and 1.00 and 0.97 for BW. The sensitivities were 0.85 and 0.81 for LF, 0.89 and 0.85 for LFpen, 0.74 and 0.85 for FC, 0.93 and 0.52 for ICDAS and 0.29 and 0.44 for BW at D2 and D3, respectively. The accuracy values were higher for LF, LFpen and ICDAS at D1 and D3 thresholds. The area under de ROC curve varied from 0.645 to 0.949 and confirmed the good performance of the methods. It can be concluded that LF, LFpen,
FC and ICDAS presented good validity in detecting occlusal caries in vivo. However, BW showed good validity to detect only caries in dentine.

Funding: Fundação de Amparo à Pesquisa no Estado de São Paulo (FAPESP, Processo 2009/00218-0) and Coordenação de Aperfeiçoamento de Pessoal de Nível Superior (CAPES).

\section{8 \\ Quantification Algorithm of Occlusal Caries for Light-Induced Fluorescence Images \\ E. de Josselin de Jong ${ }^{\mathrm{a}-\mathrm{c}, *}$, D. Inaba ${ }^{\mathrm{d}}$, M.H. van der Veen ${ }^{\mathrm{c}}$ \\ e.dejosselindejong@inspektor.nl \\ ${ }^{a}$ Research Systems B.V., Amsterdam, The Netherlands; \\ bschool of Dental Sciences, University of Liverpool, UK; \\ 'Academic Centre for Dentistry Amsterdam (ACTA), Amsterdam, The Netherlands; ${ }^{d}$ Department of Oral Health and Development Sciences, Iwate Medical University, Morioka, Japan}

Previously we described a simple blue light in combination with an optical filter to visualize occlusal caries lesions including deep dentinal ones underneath seemingly intact enamel. Based on this a digital camera fitted with the same optical filter and blue light excitation spectra was developed called QLF-D. A program was written to perform automatic analysis. Occlusal fluorescence images of 8 extracted molars were collected with QLF-D. For each tooth pixel a 4th degree polynomial fit was made though points determined by its red and green values. From this set all pixels were excluded were the red value was higher than the polynomial fit value. A second polynomial fit function was made through the new pixel set, which is assumed to represent the pixel values of sound tooth tissue. For each tooth pixel the red fluorescence factor $\Delta \mathrm{R}_{\text {pix }} \equiv \mathrm{R}_{\text {pix }} / \mathrm{R}_{\text {sound }}$ was calculated where $\mathrm{R}_{\text {pix }}$ was its red component and $\mathrm{R}_{\text {sound }}$ the value of the second polynomial fit. A cloud of pixels, aimed to exclude stains and calculus/white spot areas, was assembled using the following exclusion criteria: $\Delta R_{p}>K_{\text {stain }}$ and $\left(\mathrm{G}_{\mathrm{p}} / \mathrm{G}_{\mathrm{s}}\right)<\mathrm{K}_{\text {calc/whspot }}$. Here $\mathrm{K}_{\text {stain }}$ and $\mathrm{K}_{\text {calc/whspot }}$ were constants. A subsurface caries parameter $\Delta S$ was introduced and defined as the mean $\Delta \mathrm{R}_{\text {pix }}$ in the remaining cloud of pixels. For the 8 teeth examined we optimized the values $\mathrm{K}_{\text {stain }}$ and $\mathrm{K}_{\text {calc/whspot }}$ to 1.50 and 0.90 respectively to exclude stains and white spot/calculus. $\Delta S$ is potentially a measure for the presence of porfyrines under the tooth surface. It is also postulated that $\Delta \mathrm{S}$ a measure for the depth of the caries lesion under the enamel surface which is independent of stains and white spot/calculus presence on the tooth surface.

Funding: Inspektor Research Systems B.V. 


\section{9}

Red Fluorescence Detection from the Occlusal Aspect of Extracted Molars Related to Fluorescence Depth Profile

D. Inaba ${ }^{\mathrm{a}, *}$, E. de Josselin de Jong ${ }^{\mathrm{b}-\mathrm{d}}$, M.H. van der Veen ${ }^{\mathrm{d}}$

d.inaba001@gmail.com

aDepartment of Oral Health and Development Sciences, Iwate Medical University, Morioka, Japan; ${ }^{\text {In }}$ spektor Research

Systems B.V., Amsterdam, The Netherlands; ' School of Dental

Sciences, University of Liverpool, UK; ${ }^{\mathrm{d} A c a d e m i c}$ Centre for

Dentistry Amsterdam (ACTA), Amsterdam, The Netherlands

Recently we have developed a novel quantitative light-induced fluorescent system (QLF-D, Inspektor Research Systems B.V.) which enables us to capture green and red fluorescence at extremely high resolution. The aim of this study was to examine the red fluorescent surface intensity on the occlusal surface of molars with the depth profile of the red fluorescence. Occlusal red fluorescence images of 8 extracted human molars (\#1-8) were collected using the QLF-D system fitted with the red-color specific optical filters and blue light excitation. Then mesio- distal crosssectional planes including central pit were observed by QLF-D for depth of red fluorescence. The depth of red was rated 0: invisible; 1: limited to enamel; 2 : involved dentine; 3 : cavity. On the occlusal surface images the mean red fluorescence intensity $(\Delta S)$ was calculated, with respect to sound enamel. The range of $\Delta S$ values was 1 to 8.9 and $\Delta \mathrm{S}$ correlated significantly with depth rank with $\mathrm{r}=$ 0.78 (Spearman rank correlation, $\mathrm{p}<0.05$ ). The molars without red fluorescence in the sectional surfaces showed mean $\Delta \mathrm{S}$ value of $1.23(\mathrm{n}=3)$; molars category 1 had $\Delta S$ value of 2.7 ; molars category 2 had $\Delta S$ value of 2.7; molars category 3 had $\Delta S$ value of 5.75 In conclusion, the data suggest that the red fluorescence observed from the occlusal aspect of extracted molars has good agreement with actual depth at which fluorescence is found in hemi sections.

\section{0}

\section{Comparison of Measurements between} Conventional QLF-Pro and Newly Developed QLF-D

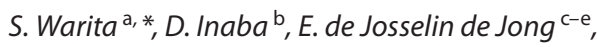
M.H. van der Veen ${ }^{\mathrm{e}}$

saciwari@tky.ndu.ac.jp

aDepartment of Pediatric Dentistry, Nippon Dental University, Tokyo, 'bepartment of Oral Health and Development Sciences, Iwate Medical University, Morioka, Japan; 'Inspektor Research Systems B.V., Amsterdam, The Netherlands; ' ${ }^{\text {S }}$ chool of Dental Sciences, University of Liverpool, UK; ${ }^{\mathrm{e} A c a d e m i c}$ Centre for Dentistry Amsterdam (ACTA), Amsterdam, The Netherlands

The conventional QLF-Pro has been upgraded to the QLF-D by applying a 15.5 Mpixels SLR (EOS 500D, Canon) and LED illumination source. The aim of this study was to examine fluorescent aspects of enamel lesions in comparison with conventional QLF-Pro. Bovine enamel blocks $(\mathrm{n}=14)$ were demineralized in a
$0.1 \mathrm{M}$ lactic acid solution ( $\mathrm{pH} 4.5$ ) for $48 \mathrm{~h}$, and then measured for $\Delta \mathrm{F}$ values (fluorescent reduction rate, \%) by QLF-Pro and QLF-D in a standardized conditions of illumination and optical setting. To evaluate whole systematic error, all the procedures from image capturing to $\Delta \mathrm{F}$ measurement were repeated 10 times independently by one operator using QLF-D for randomly selected 3 samples. In addition, 10 repetitions of $\Delta \mathrm{F}$ measurements by the QLF software were performed for each of 3 fluorescent images taken by the QLF-D to estimate measurement reproducibility. The $\Delta \mathrm{F}$ values from QLF-D for 14 samples (17.9 \pm 4.74\%) were statistically similar ( $\mathrm{p}=0.586$, unpaired t test) to the measurements from QLF-Pro (18.8 $\pm 4.45 \%)$. The $\Delta \mathrm{F}$ values for 3 samples were 26.8 $\pm 0.64 \%(\mathrm{CV}=2.4 \%), 15.98 \pm 0.75 \%(4.7 \%)$ and $16.29 \pm 0.83 \%$ (5.1\%) indicating that the systematic error of QLF-D processing was less than $5.1 \%$ for $\Delta \mathrm{F}$ values. Ten repetitions of $\Delta \mathrm{F}$ measurement resulted in $\mathrm{CV}$ values of $4.9 \%$ at most. In conclusion, the novel QLF-D system is fully compatible with the conventional QLF-Pro for fluorescent assessment and the measurement procedure of QLF-D has acceptable reproducibility in vitro.

\section{1 \\ Desktop X-Ray Micro CT Imaging Compared with Conventional Histological Sectioning as a Gold Standard in Cariology \\ C. Boca ${ }^{a}$, B. Truyen ${ }^{\text {a }}$ L. Henin ${ }^{\text {b }}$, A.G. Schulte ${ }^{\text {, }}$ V. Stachniss ${ }^{\text {d, }}$ N. Declerck ${ }^{\mathrm{e}}$, J. Cornelis a , P. Bottenberg ${ }^{\mathrm{b}, *}$ \\ pbottenb@vub.ac.be \\ Departments of aElectronics and Informatics, and ${ }^{b}$ Restorative Dentistry, Vrije Universiteit Brussel, Belgium; 'Department of Conservative Dentistry, Universität Heidelberg, ${ }^{\mathrm{d}}$ Department of Conservative Dentistry, Universität Marburg, Germany; ${ }^{\mathrm{e}}$ Micro CT Research Group, Universiteit Antwerpen, Belgium}

Conventional histological validation is a generally accepted gold standard in caries studies. Besides demanding complex laboratory processing, about $80 \%$ of the tooth material is lost irreversibly. X-ray Micro Computer Tomography (XMT) is a high-resolution, non-destructive imaging technique, not requiring elaborate specimen preparation. Desktop XMT systems significantly reduced cost and complexity of the technique. The aim of the present study is to compare both methods for categorical caries gradation and assessment of lesion depth and size. 148 extracted human premolars and molars, encompassing lesions of different extent, were selected. All teeth were individually mounted on a support to ensure identical orientation during XMT and histological preparation. After obtaining XMT images with spatial resolution of $18 \mu \mathrm{m}^{3}$ (Skyscan1076, Belgium), all teeth were embedded in resin and serially sectioned, with digital photographs being taken. Custom registration software allowed accurate correlation of histological sections and XMT slices. Images were displayed in randomized order on a $21^{\prime \prime}$ CRT monitor. Four observers scored lesion category, and marked central depth as well as lesion area, using a proprietary graphical user interface.

According to XMT $76 \%$ of the surfaces were sound, $12 \%$ had enamel and $12 \%$ had dentine caries. Histology showed $69 \%$ of 
sound surfaces, $15 \%$ with enamel caries, and $16 \%$ with dentine caries. Spearman's correlation coefficient for categories was 0.81 $(p<0.0001)$. Correlation between both techniques was 0.720 for central depth, and 0.757 for lesion area $(p<0.0001)$. Bland-Altman plots revealed that $84 \%$ of the data points were within $2 \mathrm{SD}$, with $65 \%$ total agreement between both techniques. XMT and histology demonstrated good agreement, although both techniques are based on distinct decision criteria. Due to its nondestructive nature and comparatively simple specimen manipulation, desktop XMT imaging is a potentially promising alternative to histology as a gold standard in cariology.

Supported by VUB research fund, FWO Vlaanderen.

\section{2 \\ Optical Coherence Tomography Images of Caries Analyzed by Signal Attenuation}

A.M.A. Maia ${ }^{\mathrm{a},}{ }^{*}$, A.Z. Freitas ${ }^{\mathrm{b}}$, A.S.L. Gomes ${ }^{\mathrm{a}}$

anamarlyamaia@gmail.com

aUniversidade Federal de Pernambuco, Recife, ${ }^{b}$ Instituto de

Pesquisas Energéticas e Nucleares, Sao Paulo, Brazil

The aim of our research was to improve the images generated by an Optical Coherence Tomography (OCT) set up as a method for early caries detection. The OCT images that are based on quantitative measurements of the backscattered light intensity as a function of depth into the analyzed region can be described through the attenuation signal. The OCT is a good technique to be applied to caries detection because the technique is well suited for detecting changes in optical scattering, tissue polarization, and refractive index due to morphological alterations within samples. The OCT images are formed by a straight-line collection of adjacent A-scans, plotted as a two-dimensional cross section, called B-Scan. Our OCT system (Thorlabs) was coupled to a micrometer translation stage to scan an area of $2 \times 2.5 \mathrm{~mm}$ of sound and carious enamel surface in $0.5 \mathrm{~mm} / \mathrm{s}$. It was analyzed each signal of all B-Scan that is representative of a linear sequence from adjacent spots located 6 um apart resulting in a point attenuation. The calculation of each point attenuation coefficient provides the observation of the distribution of the scattering aggregates changes representing an AScan. The optical attenuation at $930 \mathrm{~nm}$ was analyzed by acquiring 350 parallel OCT images at 6 um intervals of each scan. All images were processed by software developed in LabView environment, and each attenuation value was projected onto a $2 \mathrm{D}$ map that gives information of different optical characteristic of the depth tissue evaluated. The image shows micrometric details as cracks, and even Hetzius streaks of enamel, and also makes easy the evaluation from professionals that are not adapted to the technique.

Supported by Capes, CNPq and PRONEX - FACEPE.

\section{3 \\ Detection of Artificially Induced Secondary Caries Using Different Methods under Simulated Clinical Conditions}

C. González-Cabezas a, * M. Ando b, K. Arab c

carlosgc@umich.edu

aUniversity of Michigan, Ann Arbor, and ${ }^{b}$ Indiana University

School of Dentistry, Indianapolis, USA; ' King Faisal Specialist

Hospital and Research Center, Riyadh, Kingdom of Saudi Arabia

The aim of this investigation was to evaluate several detection methods [visual/tactile, radiography, fiber-optic transillumination (FOTI) and digital imaging fiber-optic transillumination (DIFOTI)] in the detection of secondary caries in approximal gingival margins of resin-matrix composite restorations under simulated clinical conditions. MOD resin-matrix composite restorations were placed in 120 extracted premolars and molars. Each of the two gingival margins of each specimen was assigned to one of four groups; three to have caries lesions developed; one be kept as sound margin. Two of the groups have cavities with different depths (reaching DEJ or extending about $2 \mathrm{~mm}$ into dentin) created with carbide burs. Specimens were incubated with Streptococcus mutans A 32-2 (serotype $c$ ) in TSB $+5 \%$ sucrose for three weeks and caries lesions developed at the exposed gingival margins (three of the groups). Afterwards, teeth were mounted in 10 plastic manikins (20 typodonts) and examined by three examiners under clinical conditions using the four caries detection methods. Then, specimens were sectioned though the lesions, and examined under a digital stereomicroscope for lesion extension severity scoring. Results showed that DIFOTI had the highest agreement between examiners, followed by radiographs. The likelihood ratios were powerful for all methods ( $>10$ for positive; $\leq 0.2$ for negative). The area under the ROC curve was high $(>0.80)$ for all methods, for all examiners. DIFOTI had significantly higher accuracy than the other methods ( $\mathrm{x} \leq 0.0005)$ and radiography and FOTI had significantly higher accuracy than visual/tactile ( $p \leq 0.0009$ ). Examiner repeatability showed good agreement for all methods using this model (weighted Kappa range was $0-75-0.95$ for both intra- and inter-examiner). In conclusion, this new model seems to be a good alternative to clinical studies for the screening of secondary caries detection methods.

Supported by Indiana University School of Dentistry.

\section{4 \\ Impact of 'Independent Data' Using Fluorescence Methods for Occlusal Caries Diagnosis}

\author{
A. Jablonski-Momeni * H.M. Schipper, S.M. Rosen, \\ M. Heinzel-Gutenbrunner, V. Stachniss, K. Pieper \\ momeni@staff.uni-marburg.de \\ Dental School, Philipps University of Marburg, Germany
}

Occlusal carious lesions can occur at different discrete sites and be of different appearance and severity. In assessing diagnostic accuracy, the presence of multiple lesions on an occlusal sur- 
face may cause problems for evaluating individual lesions and the group of lesions is not statistically independent. This study aimed to evaluate whether there is an effect of 'independent data' on the performance of fluorescence based devices and the reproducibility of the measurements when used on occlusal surfaces. 36 permanent extracted teeth (2-3 investigation sites on each occlusal surface, $\mathrm{n}=82$ ) were chosen. Two investigators examined the sites using DIAGNOdent pen (LF) and VistaProof (FC). Each investigation site was measured independently in a random order twice within one day. The teeth were serially sectioned for assessment of lesion depth. Intra-class-correlation coefficients (ICC) for intra- and inter-examiner reproducibility and the areas under the ROC-curve (AUC) were calculated for all investigation sites. Additionally, one investigation site per tooth was randomly selected (independent data, $\mathrm{n}=36$ ). To test whether reproducibility and performance differ systematically between independent data and measurements including all investigation sites, the effect size (Cohen's d) was calculated. The results for all investigation sites were: ICC: LF 0.91-0.97, FC 0.83-0.94; AUC: LF 0.71-0.82, FC 0.740.82 . The results for the independent ere: ICC: LF $0.83-0.98$, FC 0.79-0.91; AUC: LF 0.58-0.72, FC 0.63-0.71. Comparing the ICC for all data and for the randomized selection, a small effect for the LF (effect size 0.11-0.5) and no effect for the FC (0.07-0.2) occurred. AUC for the whole sample and the randomized data showed small effects for LF and FC (0.17-0.25). Measuring multiple sites on teeth with fluorescence devices moderately influences performance compared to one site being investigated.

Supported by Dürr Dental, Bietigheim-Bissingen, Germany.

\section{5 \\ Validation of an Optical Method and Sensor for Assessment of Caries Lesion Activity \\ K.W. Neuhaus ${ }^{\text {a, * }}$, B. Nyvad ${ }^{\text {b }}$, J. Frohn ${ }^{c}$, A. Lussi $^{\text {a }}$, L. Jaruszewski ${ }^{\mathrm{d}}$ \\ klaus.neuhaus@zmk.unibe.ch \\ aSchool of Dental Medicine, University of Bern, Switzerland;

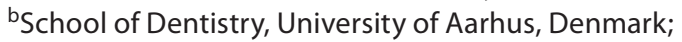

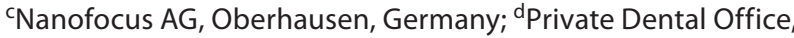 \\ Hildesheim, Germany}

Aim: To validate the assessment of lesion activity by means of an experimental optical sensor measuring reflexion intensity. Methods: Fourty teeth with enamel lesions were selected from a pool of extracted teeth, stored in $1 \%$ chloramine solution at $4^{\circ} \mathrm{C}$. Each tooth was cut in a clinically sound half and a half with a noncavitated lesion. After gentle plaque removal with $\mathrm{NaOCl}$ for $30 \mathrm{~s}$ and rinsing with tap water, the teeth were kept humid. The lesions were then photographed at $6.25 \times$ magnification and one measuring point was chosen per lesion and indicated with an arrow on a printout. Independently from each other, visual assessment (VA), optical assessment using an experimental sensor (ES) and confocal profilometry $(\mathrm{CP})$ of the chosen spot was performed. ES is a handheld confocal chromatic sensor rendering natural numbers $0-50$ indicating the percentage of reflexion intensity. The results were compared by calculating the $95 \%$ confidence intervals for ES and OP. Not overlapping CIs indicate a statistically significant differ- ence. Results: Both ES and CP rendered significantly different values comparing lesions visually scored as active or inactive. For ES the $95 \%$ CIs for lesions scored active and inactive were [11.5; 19.5] and $[24 ; 29]$, respectively $(\mathrm{p}<0.05)$. For $\mathrm{CP}$, the corresponding $95 \%$ CIs were $[0.56 \mu \mathrm{m} ; 1.05 \mu \mathrm{m}]$ and $[0.36 \mu \mathrm{m} ; 0.5 \mu \mathrm{m}]$, respectively $(p<0.05)$. ES values of the clinically sound control teeth were significantly different from active lesions, but not from inactive lesions. CP values of the control surfaces were not significantly different from active/inactive lesions. Conclusion: Within the limits of this study, the experimental sensor can be regarded as a tool for quantitative enamel lesion activity assessment. Practical aspects of the ES device should be considerably improved.

\section{6 \\ Can New Methods Improve the Detection of Proximal Carious Lesions in Primary Molars?}

N. Chawla *, L.B. Messer, D.J. Manton

ljbm@unimelb.edu.au

Melbourne Dental School, Melbourne, Australia

Aims: To compare and contrast eight available carious lesion detection methods (visual inspection using ICDASII, digital radiography, four transillumination methods, two methods based on laser-fluorescence) with the gold standard (histology) to determine the method with the highest accuracy for detecting proximal carious lesions in extracted primary molars. Materials and Methods: Extracted primary molars $(n=140)$ were stored in $0.02 \%$ chlorhexidine solution and mounted in light-cured impression tray material in pairs. Three examiners used eight detection methods to assess proximal carious lesions. Five damaged teeth were discarded. The teeth $(n=135)$ were sectioned, seriallyground, and examined under light microscopy using Downer's histological (HST) criteria as the gold standard. Intra- and interexaminer reliability, agreement with HST, specificity, sensitivity, ROC curves and areas under the curve (AUC; Az) were calculated. Results: For the main study, transillumination (SDI instrument without collimator) had the highest inter-examiner reliability (0.53); digital radiography had the highest intra- and inter-examiner reliability $(0.76,0.63)$. Combining the measures of specificity and sensitivity into the area under the receiver operating characteristic curves (AUC), visual inspection had the highest value for detecting enamel and dentinal carious lesions $(\mathrm{Az}=0.74)$, and digital radiography had the highest value for detecting dentinal carious lesions only $(\mathrm{Az}=0.86)$. Conclusions: Enamel and dentinal carious lesions on proximal surfaces of primary molars were detected most accurately by visual inspection followed by digital radiography, and dentinal carious lesions were detected most accurately by digital radiography followed by visual inspection. The four transillumination methods were more accurate in detecting dentinal carious lesions only than enamel and dentinal carious lesions, but did not achieve the accuracy of visual inspection and digital radiography. The two laser fluorescence methods studied were judged to be of little diagnostic value.

Supported by Melbourne Dental School, the University of Melbourne. 


\section{7}

\section{Using PTR-LUM ('The Canary System') for in vivo Detection of Dental Caries: Clinical Trial Results}

K. Sivagurunathan ${ }^{\text {a }, ~ S . H . ~ A b r a m s ~}{ }^{\text {a, }}$, J. Garcia ${ }^{\text {a }}$, A. Mandelis ${ }^{\text {a }}$,

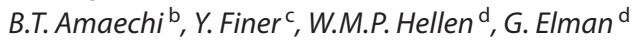

dr.abrams4cell@sympatico.ca

${ }^{a}$ Quantum Dental Technologies, Center for Advanced Diffusion

Wave Technologies (CADIFT), Toronto, Canada; ${ }^{\text {b University }}$

of Texas Health Science Center, San Antonio, USA; ' ${ }^{\text {FFaculty of }}$

Dentistry, University of Toronto, and d Cliffcrest Dental Office,

Toronto, Canada

Objective: Using Frequency Domain Photothermal Radiometry (PTR) and Modulated Luminescence (LUM) diagnostics, Quantum Dental Technologies, Inc., (QDT) has developed and tested a portable PTR-LUM instrument (The Canary ${ }^{\mathrm{TM}}$ Dental Caries Detection System) detecting artificial dental caries in several in-vitro studies. The aim of the present clinical trial was to determine the safety and in-vivo application of this device for clinical detection of early caries. Methods: In this study, PTR, LUM amplitude (A) and phase (P) responses at various modulation frequencies from healthy and carious dental enamel (ICDAS 0-6) were measured. A clinical trial was done using The Canary ${ }^{\mathrm{TM}}$ (prototype 1), under the approval of Health Canada. Over 500 regions on healthy tooth surfaces of 50 subjects were used to construct a healthy baseline for each output channel (1-PTR-A, 2-PTR-P, 3-LUM-A and 4-LUM-P). While PTR-A and PTR-P were used to detect near-surface and subsurface lesions, LUM-A and LUM-P were used to detect near-surface lesions. Results: The Canary ${ }^{\mathrm{TM}}$ did not cause any adverse events or soft or hard tissue trauma. There was no difference in signal from wet or dry tooth surfaces; anterior and posterior healthy tooth surfaces provided the same signal, and the presence of surface stain and biofilm did not affect the signal from healthy tooth surfaces. In carious enamel, we observed a clear shift from the baseline in both PTR (PTRA increased up to 6-230\%, PTR-P shifted up to 5-25\%) and LUM (LUM-A decreased up to 7-85\%, LUM-P shifted up to 1-9\%) depending upon the type, depth and nature of the lesion. Conclusion: Results from this first clinical trial showed that the Can$\operatorname{ary}^{\mathrm{TM}}$ is safe, discriminates healthy and carious enamel.

Supported by The Health Technology Exchange (HTX) Project HTX 2007-13.

\section{8 \\ The Caries Assessment Spectrum Index: Combination of ICDAS II, PUFA and DMF Indices}

\author{
J.E. Frencken ${ }^{\mathrm{a}, *}$, R.G. Amorim ${ }^{\mathrm{b}}$, J. Faber ${ }^{\mathrm{b}}$, S.C. Leal ${ }^{\mathrm{b}}$ \\ j.frencken@dent.umcn.nl \\ ${ }^{a}$ Global Oral Health, Radboud University Nijmegen,

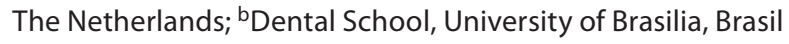

This presentation describes the rational and content of a novel caries assessment index that is termed: Caries Assessment Spectrum (CAS). It combines the ICDAS II and PUFA indices, and the $\mathrm{M}$ - and F-components of the DMF index. The CAS index consists of assessing stages of carious lesions progression in enamel, dentine and the pulp, together with teeth Missing and Filled due to dental caries. The index is expressed as EDPMF-T/S. The word 'spectrum' depicts what is considered the main strength of the new index - its usefulness to describe the complete range of stages of carious lesion progression from no carious lesion through lesions in enamel and dentine, to the advanced stages of carious lesion progression in pulpal and tooth surrounding tissue in the public oral health setting. Furthermore, treatment requirements are better derived from the CAS index than from the ICDAS II, PUFA or DMF indices. The CAS index has been used in a child population in Brasil in a response to difficulties encountered in reporting study outcomes from using PUFA and in particular, ICDAS II. How to report results from using the CAS index will be presented. A DMF score can easily be deduced from the CAS score which secures the use of existing DMF scores all over the world. Both the individual index codes at enamel, dentine and pulpal level as well as combinations are reported, as can the consequences of advanced stages of untreated cavitated carious lesions with CAS. The use of the CAS index is very promising and should be used in different age groups in different countries and cultures. 


\section{Caries Research}

\section{Session 8 Caries Risk}

\section{9 \\ Oral Health Status and Treatment Need of 41 Hospital Patients with Rheumatoid Arthritis \\ R. Boudas ${ }^{\mathrm{a}, *}$, L. Bigeard ${ }^{\mathrm{a}}$, L. Brisset ${ }^{\mathrm{a}}$, J. Sibilia ${ }^{\mathrm{b}}$, A.M. Musset ${ }^{\mathrm{a}}$ \\ bigeardl@msn.com \\ aDental Faculty, ${ }^{\mathrm{b}}$ Faculty of Medicine, Strasbourg, France}

Objectives: To assess oral health state of patients with rheumatoid arthritis (RA) treated in the University Hospital of Strasbourg (France) and to develop a protocol of specific preventive dental care. Design: A transversal descriptive epidemiologic study to describe oral conditions, salivary parameters and craniomandibular dysfunctions. Subjects: The 41 patients hospitalized for RA treatment monitoring from April to October 2008, aged from 20 to $80(80 \%[0.68 ; 0.92]$ female, sex ratio $=0.24) .70 \%[0.56$; 0.84 ] of the population was aged from 50 to 69 years $(\mathrm{m}=57.8$; median age $=61$; s.d. $=14.82$; Variation Coefficient $=0.256)$. Results: The mean DMFT: 20.95 [18.6; 23] (s.d. = 7.42) and DMFS: 83.9 [72; 95.8] (s.d. $=38.8$ ) were high. The 50 to 64 aged subjects presented a mean DMFT of $21.32[12.4 ; 30.22]$ and a mean DMFS of 89 [52;126]. The 65 to 79 years-old subjects presented a mean DMFT of 20.58 [8.9; 32.2] and a mean DMFS of $75.66[33 ; 118]$. There was no significant difference between the two age groups $(\mathrm{p}<0.05)$. Following data were calculated on the whole sample. Sialometry showed that $22 \%$ had low and $22 \%$ moderate salivary flow, $58.5 \%$ a high level $\left(>10^{5} \mathrm{CFU} / \mathrm{ml}\right)$ of Streptococus mutans and $49 \%$ presented a diminution of the salivary buffering capacity. A high prevalence of clinical signs of cranio-mandibular dysfunctions was observed $(46.3 \%$ showed a limitation of mouth opening, 52\% clicking and/or cracking noises and 37\% suffering from TMJ pains. Conclusion: The RA patients were at high carious risk. They cumulated unfavourable salivary conditions with functional impairment responsible of defective oral hygiene.

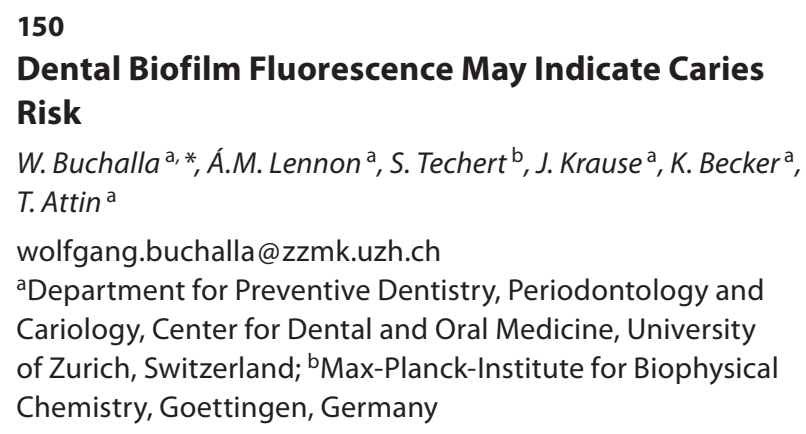

This study explored dental biofilm/plaque fluorescence and its potential for caries risk assessment. 'Clinical findings' were recorded from 65 volunteers (age 18-73): Dental visits (frequency); education; oral hygiene; fluoride exposure; nutrition; salivary flow rates, $\mathrm{pH}$, calcium, phosphate, buffer-capacity $\left(\right.$ CRT-buffer $\left.{ }^{\circledR}\right)$, bacteria (Dentocult $\left.{ }^{\circledR}-\mathrm{SM} / \mathrm{LB}\right)$; lactate $\left(\right.$ Clinpro $^{\mathrm{TM}}$ Cario L-Pop ${ }^{\mathrm{TM}}$ ); plaque index (PI; Silness J, Löe H: Acta Odontol Scand 1964;22: 121-135); plaque-pH; PSI; DMFS; initial and active caries. A pooled sample of mature plaque from each volunteer was subjected to fluorescence-spectrophotometry at $405 \mathrm{~nm}$ excitation. Following professional tooth cleaning and three days without oral hygiene, the volunteers were re-examined concerning PI, plaque$\mathrm{pH}$, and plaque fluorescence (second examination). A red-overgreen ratio (RGR) comprising spectral intensity integrated from 565-720 nm (green) and 490-540 nm (red) was calculated. Using the Cariogram (Bratthall D: Eur J Oral Sci 1996;104:486-491), the chance-values from $0-33 \%$ were defined as 'high risk', $34-67 \%$ as 'moderate risk' and 68-100\% as 'low risk'. Correlation and regression were analysed between clinical findings and RGR. All dental biofilm samples showed a native peak in the green spectrum and several peaks in the red spectrum that resembled porphyrin fluorescence. Emission spectra from 'high risk' volunteers showed more intense fluorescence in the red spectrum compared to 'moderate risk' and 'low risk' volunteers. Comparing mature to 3-dayold plaque, fluorescence intensity in the red spectrum decreased in 'high risk', but increased in 'low risk' volunteers. Among clinical findings correlating with RGR, at the first examination the following were significantly $(\mathrm{p}<0.05)$ associated with RGR by bivariate linear regression: Age, stimulated salivary flow rate, lactate, LB and SM, PI, PSI, DMFS, and active caries. At the second examination, SM, resting saliva calcium, PI, active and initial caries lesions, and chance-values were related. The fluorescence fingerprint of dental plaque may be suitable for caries risk assessment. 
4.492, $\mathrm{p}=0.034)$ reduced the chances of experiencing ECC. The presence of PRP IB-4 significantly increased caries experience (B $=2.731$, S.E. $=1.295, \mathrm{~W}=4.449, \mathrm{p}=0.035)$. In addition, age $(\mathrm{B}=$ 0.041$, S.E. $=0.018, \mathrm{~W}=5.388, \mathrm{p}=0.020)$ and $\mathrm{MS}$ counts $(\mathrm{B}=0.141$, S.E. $=0.067, \mathrm{~W}=4.381, \mathrm{p}=0.036)$ increased caries experience, however gender was not associated with dental caries $(B=-0.074$, S.E. $=0.474, \mathrm{~W}=0.024, \mathrm{p}=0.877)$. Our results suggest that presence of specific peptides in saliva of CF or CE children in early childhood predisposes to a higher or lower risk of caries experience.

Supported by FUNCAP (Association which supports research in the state of Ceara, Brazil).

\section{4 \\ Early Childhood Carious Lesions Progression in Primary Dentition over a 33-Month Period in Cree Indian Children}

J. Véronneau

jacques.veronneau@mcgill.ca

McGill University, Montreal, Canada

Caries in primary teeth is an ongoing issue in children's dental health. Relatively little is known on rates of initial carious lesions progression through the enamel or the dentin. This longitudinal study was designed to assess visually, the rate of initial carious lesion progression between the ages of 21 and 54 months among Cree Indian children. As part of a single blind, randomized, controlled trial to assess a dental health intervention using fluoride varnish, 170 young children aged 21 months, were initially randomly allocated to a group control. Children had no trial intervention and were exposed to normal care control group for a mean period of 33 months. At baseline and outcome, $\mathrm{d}_{1-4} \mathrm{efs}$ (number of decayed, missing [due to caries] and filled tooth surfaces) at noncavitated level, was measured in the children by a total of 3 trained, calibrated dental hygienists blinded to the group allocation. The evaluation was carried out in community centres or daycares using an illuminated mirror, an explorer and $2 \times 2$ gauze pads on a table with mattress or a regular chair. The teeth were cleaned prior to examination. Carious lesions rates (percentage) of progression on all dental surfaces were measured from initial stage $\left(d_{1}\right)$ to others $\left(d_{2-4}\right)$. A total of 8,777 surfaces were considered. Among all surfaces, 2,276 (25.9\%) had lesion experience during the study period. On all initial carious lesions at baseline, lesion progressed $50 \%$ of the time and regressed $40 \%$ of the time during the study period. These results suggest that initial carious lesion in vulnerable children tend to progress about half of the time between the age of 2 and 4 years old.

Supported by the James Bay Public Health Authorities, Cree Health Board, Quebec, Canada.

\section{5 \\ Effect of Hyperbaric Oxygenation on Post-Radiation Xerostomia in Patients with Teeth and in Edentulous Patients}

K. Cankar*, Z. Finderle, J. Jan

ksenija.cankar@mf.uni-lj.si

Medical Faculty, University of Ljubljana, Slovenia

In our previous study we observed that hyperbaric oxygenation (HBO) therapy improves salivary function and decreases the quantity of cariogenic bacteria in patients exposed to radiotherapy of the salivary glands. There is no data on the influence of the teeth present on this improvement. Our aim was to evaluate the influence of $\mathrm{HBO}$ therapy on post-radiation xerostomia in patients with teeth and in edentulous patients. Eight patients with teeth (20 \pm 8 teeth present, with dentinal carious lesions) and eight matched edentulous patients were included. Examinations were performed at baseline before and after 20 daily $\mathrm{HBO}$ therapies in a hyperbaric chamber at 2.5 ATA, where patients breathed $100 \%$ oxygen for $90 \mathrm{~min}$ each day. The measurements of salivary flow, $\mathrm{pH}$, and colony densities of $S$. mutans (SM), Lactobacillus $(L B)$ and C. albicans (CA) in stimulated saliva were performed using Orion Diagnostica (Finland) tests. There were no significant differences in the measured parameters between the two groups at baseline. After $\mathrm{HBO}$ therapy salivary flow increased in patients with teeth from $0.38 \pm 0.2 \mathrm{ml} / \mathrm{min}$ to $0.7 \pm 0.3(\mathrm{p}=0.031)$ as well as in edentulous patients from $0.11 \pm 0.1$ to $0.86 \pm 0.2 \mathrm{ml} / \mathrm{min}$ (paired t test; $\mathrm{p}=0.006$ ). Salivary $\mathrm{pH}$ increased significantly only in edentulous patients from $5.8 \pm 0.2$ to $6.5 \pm 0.2(\mathrm{p}=0.016)$ and not in patients with teeth. SM colony density decreased in patients with teeth and in edentulous patients from $10^{5}$ to $10^{4} \mathrm{CFU} / \mathrm{ml}$ (Wilcoxon test; $\mathrm{p}=0.031$ vs. $\mathrm{p}=0.011$ ). However, $L B$ and $C A$ decreased from $10^{5}$ to $10^{4} \mathrm{CFU} / \mathrm{mL}$ only in edentulous patients $(\mathrm{p}=$ 0.008 vs. $p=0.021$ ), but did not change in patients with teeth. The results showed the effect of $\mathrm{HBO}$ therapy on salivary $\mathrm{pH}$ and $\mathrm{CFU}$ of $\mathrm{LB}$ and CA was more beneficial in the edentulous group of patients.

Supported by Slovenian Ministry of Science and Education (No. PO-510-381 and L3-9693-0312).

\section{6 \\ Effect of Maltitol or Xylitol Sugar-Free Chewing Gums on Salivary Parameters Related to Dental Caries Development}

V. Macioce ${ }^{\mathrm{a}, *}$, C. Thabuis ${ }^{\mathrm{a}}$, B. Zhong ${ }^{\mathrm{b}}$, S. Li ${ }^{\mathrm{b}}$, C. Lefranc-Millot ${ }^{\mathrm{a}}$, M. Berard ${ }^{c}$, D. Wils ${ }^{\text {a }, ~ L . ~ G u e r i n-D e r e m a u x ~}{ }^{\text {a }}$

valerie.macioce@roquette.com

aRoquette, Lestrem, France; 'Tongji University Medical College, Shanghai, and 'Roquette, Shanghai, China

In order to better understand the role of polyols in dental caries prevention, the effects of maltitol and xylitol chewing gums (CG) on saliva parameters were assessed in a double-blind, parallel, randomized, double-controlled study conducted in China. 
240 children aged 13-15 years were divided into four groups: three groups chewing for $10 \mathrm{~min}$ either SweetPearl-maltitol CG, Xylisorb-xylitol CG or gum base, 5 times/day for 30 days, and a control group without CG supplementation. Frequency of dental hygiene was defined, and salivary parameters (flow, $\mathrm{pH}$, free sialic acid, glucan sucrase) were evaluated at different times.

Compared to gum base, polyols CG lead to greater areas under the curves of salivary flow $(\mathrm{p} \leq 0.05)$ and $\mathrm{pH}(\mathrm{p} \leq 0.05)$. Free sialic acid concentration was reduced over study period in all gum groups (maltitol, $-0.133 \mu \mathrm{mol} / \mathrm{l}$; xylitol, $-0.118 \mu \mathrm{mol} / \mathrm{l}$; gum base, $-0.0655 \mu \mathrm{mol} / \mathrm{l}$ ), and this reduction was more important with polyols CG than with gum base $(\mathrm{p}=0.008)$. For these three parameters, maltitol and xylitol induced similar changes. However, the decrease in salivary glucan sucrase activity was more important with maltitol than with xylitol $(\mathrm{p}=0.002)$, itself showing a stronger modification than gum base $(\mathrm{p}=0.01)$. Lastly, compared to the no-gum group, gum base induced significant increases in salivary flow and $\mathrm{pH}$ and significant decreases in free sialic acid concentration and glucan sucrase activity. Moreover, none of the children reported any feeling of digestive discomfort, evaluated by questionnaire.

Therefore, this study showed that maltitol and xylitol CG induced saliva parameters modifications which are favourable to dental caries prevention. These effects might not only be due to mastication as the beneficial changes observed are stronger with polyols CG than with gum base.

Supported by Roquette.

\section{7 \\ Expression and Activity Profiles of Matrix Metalloproteinases in Bovine and Human Dentine

\author{
A.R. Hannas ${ }^{\text {a, }}$, M.T. Kato ${ }^{\text {a }}$, A.L. Leite ${ }^{\text {a }}$, B.L. Zarella ${ }^{\text {a }}$, \\ L. Tjäderhane ${ }^{b}$, M.A.R. Buzalaf ${ }^{a}$ \\ mbuzalaf@fob.usp.br \\ aBauru Dental School, University of São Paulo, Bauru, Brazil; \\ bUniversity of Oulu, Finland
}

Matrix metaloproteinases (MMPs) are endopeptidases responsible for the degradation or resorption of all components of the extracellular matrix (ECM). They are involved in the development of human dental enamel and remodeling of dentine organic matrix. MMPs-2, -8 and -9 have already been identified in human dentine. Since bovine dentine is frequently employed in in vitro and in situ studies as surrogate for human dentine, the purpose of the present investigation was to evaluate the expression and activities of MMP-2 and -9 present in human and bovine dentine. Blocks from bovine incisors and human molars were obtained from roots and crowns. They were pulverised using a cryogenic mixer mill. Dentin powder was demineralised in $0.87 \mathrm{M}$ citric acid $\mathrm{pH} 2.3$ for $24 \mathrm{~h}$ at $4^{\circ} \mathrm{C}$ under stirring. After centrifugation, the demineralised dentin powder was suspended in extraction buffer and sonicated 3 times. Samples were incubated for $24 \mathrm{~h}$ in extraction buffer. After centrifugation, the supernatant was collected and total protein concentration was measured by Lowry's method. Gelatin zymography was performed. Total activity of MMPs-
2 and -9 was determined by ELISA. Gelatinolytic activities identified as MMP-2 and MMP-9 were detected in extracts of crown and root dentin samples both from human and bovine origin. Total activities of MMP-2 were 14.85, 20.16, 14.71 and $22.92 \mathrm{ng} / \mathrm{ml}$ for bovine root, human root, bovine crown and human crown, respectively. The correspondent figures for MMP-9 were 16.71, 19.95, 21.97 and $19.46 \mathrm{ng} / \mathrm{ml}$, respectively. The results indicate that all kinds of dentin substrates tested can be used in studies involving the performance of MMPs-2 and -9.

Funding: FAPESP (07/08389-3, 07/04209-0 and 08/09857-3) and $\mathrm{CNPq}(557863 / 2008-2)$.

\section{8 \\ Infiltrants Inhibit Progression of Natural Caries Lesions in vitro}

\section{H. Meyer-Lueckel *, V. Alex, N. Buchmann, S. Paris \\ meyer-lueckel@konspar.uni-kiel.de \\ Christian-Albrechts-Universität Kiel, Germany}

The infiltration of enamel caries with light curing resins (infiltrants) might be an effective novel approach to arrest lesions under cariogenic conditions. The aim of this study was to evaluate the progression of infiltrated natural enamel caries lesions stored in a demineralizing environment in vitro. Natural enamel caries lesions (ICDAS II code: 2) of extracted teeth were etched with $15 \%$ hydrochloric acid gel for $120 \mathrm{~s}$ and subsequently infiltrated with either one of four experimental infiltrants (A: BisGMA 25\%, TEGDMA 75\%; B: BisGMA 20\%, TEGDMA 60\%, ethanol 20\%; C: TEGDMA 100\%; D: TEGDMA $80 \%$, ethanol 20\%) for $5 \mathrm{~min}$. As a positive control (PosC), lesions were covered with a flowable composite resin; whereas untreated lesions served as negative controls (NegC). Specimens of $1 \mathrm{~mm}$ thickness were prepared and exposed to a demineralizing solution ( $\mathrm{pH} 4.95)$ for 400 days. Progression of mineral losses was analyzed using Transversal Wavelength-Independent Microradiography. Median (25th; 75th percentile) baseline integrated mineral loss was $8,334 \mathrm{vol} \% \times \mu \mathrm{m}$ $(6,458 ; 13,535)$ for all lesion parts $(n=77)$ evaluated. After 400 days demineralization $\mathrm{NegC}$ showed median mineral progression of $24 \%(10 ; 56)$. Lesions of PosC, C, and D revealed almost no progression, whereas those of NegC, A, and B were significantly demineralized. It can be concluded that infiltrants mainly consisting of TEGDMA are capable to inhibit progression of natural enamel caries lesions in vitro.

Supported by the Deutsche Forschungsgemeinschaft (PA 1508/1-1). 


\section{9 \\ Contribution of Mass Spectrometry Imaging to the Molecular Study of Human Dentin \\ C. Hirtz ${ }^{a}{ }^{*}$, V. Grausi ${ }^{\text {a }}$, A. Bouslimani ${ }^{\text {b }}$, F. Cuisinier $^{\mathrm{a}}$, C. Larroque $^{\mathrm{b}}$, $D$. Deville de Périère ${ }^{a}$ \\ christophe.hirtz@univ-montp1.fr \\ aFaculty of Odontology, University of Montpellier, \\ bIRCM, Montpellier, France}

Matrix Assisted Laser Desorption Ionisation (MALDI) imaging is a new emerging technique that allows studying spatial distribution of proteins and peptides directly on a tissue specimen. This technique involves mounting a thinly sliced tissue section onto a sample plate and applying MALDI matrix directly on top of the tissue. A two-dimensional array of MALDI mass spectra are obtained, each with a location component. Objective: The aim of this works was to develop the MALDI imaging on tooth section in order to localize precisely specific proteins or peptides. Methods: Human fresh teeth were conserved after extraction in saline solution, at $4^{\circ} \mathrm{C}$ in order to maintain tissue morphology and minimize molecular degradation. Teeth were demineralised (Nitric acid) prior to paraffin-embedded and sectioning using a microtome at ambient temperature. MALDI MS imaging was performed using a 4,800 ToF/Tof mass spectrometer (Applied Biosystem) at a laser pixel resolution of $100 \times 100 \mu \mathrm{m}$ using an MS Reflector Positive method scanning the mass range 800-4,000 amu, firing 100 laser shots per pixel. Results: Our MSI study allowed the localization of multi ionic peptides specific to the human dentin. We present here the first molecular images of three distinct human dentin peptides. Conclusion: Our results showed the potential interest of this oral tissue molecular analysis method. The perspectives are now to analyze molecular images of dental hard tissues damage that will help us to understand the process of decay at a molecular level.

Funding: ANR.

\section{0 \\ Fluorescent and Non-Linear Optical Microscopy Study of Sound and Carious Tooth Dentin - Comparative Study \\ I. Panayotov ${ }^{\mathrm{a}, *}$, G. Romieu ${ }^{\mathrm{a}}$, B. Levallois ${ }^{\mathrm{a}}$, M. Martin ${ }^{\mathrm{b}}$, T. Cloitre $^{\mathrm{b}}$, C. Gergely ${ }^{\text {b }}$, F.J.G. Cuisinier ${ }^{\text {a }}$ \\ ivan.panayotov@univ-montp1.fr \\ ${ }^{a}$ EA 4203 Laboratory, Faculty of Dentistry, Montpellier 1 University, bUMR 5660 Laboratory, Montpellier 2 University, France}

Fluorescence has been recently applied in different types of human tooth caries diagnostic apparatus. In this work, we used fluorescence and the non-linear optical (NLO) microscopy to investigate the dental caries patho-physiology process. The objective of this study was the characterization and the differentiation of the healthy sound and the carious dentin and the understanding investigation of the dentin collagen fluorescence variations.
Materials and Methods: We have prepared eight longitudinal slices (thickness up to $0.5 \mathrm{~mm}$ ) from two freshly extracted teeth: 4 from a sound tooth without caries and non carious alterations and 4 from a carious tooth. The samples were polished to $0.25 \mu \mathrm{m}$ and cleaned up in an ultrasound alcohol bath for $5 \mathrm{~min}$. We have performed one photon fluorescence microscopy (1 PEF), using a wavelength of $480 \mathrm{~nm}$, on normal sound and caries carious dentin structures. NLO microscopy was used to imaging of dentin was provided by means of two photon florescence and second harmonic generation (2 PEF/SHG), using an incident wavelength of $800 \mathrm{~nm}$. Results and Conclusion: With the NLO microscopy images we have visualized groups of collagen fibers constituents of the healthy dentin extracellular matrix. We have also succeeded to make a differentiation between healthy and carious tooth dentin structure. The visualization of the three dimensional structure of the samples and the optical sectioning capacity inherent to the non linear optical microscopy technique could offer a new perspective of development to the caries diagnostic.

\section{1 \\ Carbonate Content of Enamel in Hypomineralised Molars \\ F.A. Crombie*, D.J. Manton, I. Zalizniak, E.C. Reynolds \\ djmanton@unimelb.edu.au \\ Melbourne Dental School, University of Melbourne, Australia}

The aim of this in vitro study was to investigate the relative carbonate content of enamel in teeth affected by molar hypomineralisation $(\mathrm{MH})$. Specimens were prepared from extracted first permanent molar teeth diagnosed clinically with $\mathrm{MH}$ by sectioning with a diamond blade cut-off saw, fracturing the enamel and grinding it into a fine powder to facilitate rapid dissolution. Enamel samples $(10 \pm 5 \mathrm{mg})$ were weighed in microcentrifuge tubes $(1.5 \mathrm{ml})$ then placed into a chamber containing $5 \mathrm{M}$ hydrochloric acid solution connected to a pressure tranducer. Real-time pressure data (represented by voltage) was recorded using a laptop computer and the acid was allowed to contact the enamel sample once the chamber was sealed and the system stabilised. Raw data was adjusted to aid analysis and the observed voltage change was used to calculate the amount of carbon dioxide released from each enamel sample from which carbonate content (as \% weight) was extrapolated. Enamel samples from hypomineralised areas were found to contain $1.80 \pm 0.08$ times the amount of carbonate relative to control enamel samples. The results indicate that not only is the enamel in hypomineralised molars quantitatively low in mineral but that the quality of the mineralised tissue is also compromised with higher levels of carbonated apatite present when compared to normal enamel.

Supported by Melbourne Dental School CRC for Oral Health Science. 


\section{2 \\ Morphological Changes on Human Dentin Caused by Irradiation with a Cold Atmospheric Plasma Jet}

S. Rupf ${ }^{a},{ }^{*}$, A. Lehmann ${ }^{\text {b }}$, M. al Muhammad ${ }^{\text {a }}$, B. Schäfer ${ }^{\text {a }}$, M. Hannig ${ }^{\mathrm{a}}$, A. Schindler ${ }^{\mathrm{b}}$

stefan.rupf@uks.eu

${ }^{a}$ Clinic of Operative Dentistry, Periodontology and Preventive

Dentistry, Saarland University, Homburg, 'Leibniz Institute for

Surface Modification, Leipzig, Germany

Atmospheric plasma jets are being intensively studied with respect to potential applications in medicine. The antimicrobial efficacy of a microwave-powered non-thermal atmospheric plasma jet against adherent oral microorganisms on dentin has been demonstrated in a previous study. The aim of this study was to test the effects of cold atmospheric plasma on (1) the wettability of dentin, (2) the smear layer which appears after the preparation and effects in combination with $\mathrm{H}_{3} \mathrm{PO}_{4}$-etching (37.5\% gel), as well as (3) on carious dentin. Dentin slabs were obtained from crowns of human molars and polished (grain P2500). For the investigation of the smear layer some dentin samples were roughened using a preparation diamond $(80 \mu \mathrm{m})$. Irradiation was carried out by a non-thermal plasma source (microwave driven, 2.45 $\mathrm{GHz}$ ) mounted on a computer controlled 3-axis motion system with the following parameters: distance: $2.5 \mathrm{~mm}$; mean power: 1.5-3 W; inert gas: $\mathrm{He}$, process gases: $\mathrm{O} 2, \mathrm{~N} 2$, irradiation time: $0.3-0.9 \mathrm{~s} / \mathrm{mm}^{2}$, surface temperature: $38-44^{\circ} \mathrm{C}$. Wettability changes were determined by contact angle measuring. Surface morphology was analyzed by scanning electron microscopy (fixation and dehydrating: glutaraldehyde $2.5 \%$ in PBS, increasing series of ethanol, 1,1,1,3,3,3-hexamethyl-disilazane) in a ULTRA 55 Carl Zeiss scanning electron microscope (magnification: $500 \times-$ $16,000 \times)$. Plasma irradiation caused surface changes on dentin surfaces: (1) the wettability increased (mean contact angle for $\mathrm{H}_{2} \mathrm{O}$ untreated: $52^{\circ}$, plasma treated: $\left.9^{\circ}\right)$, (2) smear layer was reduced and dentin tubules were exposed, effects of $\mathrm{H}_{3} \mathrm{PO}_{4}$-etching were strengthened by plasma, (3) microorganisms were minimized and dentin tubules in carious dentin became visible again. Cold plasma irradiation could enable new strategies for the infiltrative treatment of dentin lesions.

Supported by the Federal Ministry of Education and Research Germany (BMBF FKZ 01 EZ 0730 and 01 EZ 0731).

\section{3 \\ Molar Incisor Hypomineralization and Dental Caries: Prevalence, Severity and Correlation between the Two Diseases \\ F. Jeremias, J.F. Souza, C.M. Costa-Silva, A.C.C. Zuanon, R.C.L. Cordeiro, L. Santos-Pinto * \\ Ispinto@foar.unesp.br \\ Araraquara Dental School, UNESP, Universidade Estadual \\ Paulista, Araraquara, Brazil}

Molar incisor hypomineralization (MIH) is a developmental disturbance concerning first permanent molars and permanent incisors. This study investigated the prevalence and severity of
$\mathrm{MIH}$ and the correlation between the two diseases in a group of Brazilian children. The study group consisted of 6-12 years old scholars, 893 from public schools and 264 from private schools. First permanent molars and all permanent incisors were examined for demarcated opacities, post-eruptive enamel breakdown, atypical restorations and extractions due to MIH (EAPD criteria, 2003). Deciduous and permanent teeth caries experience were recorded using the WHO criteria, 1997. DDE screening index (FDI, 1992) was also applied to evaluate enamel defects in deciduous teeth. Data were analyzed using chi-square test $(\alpha=5 \%)$. The prevalence of MIH was $16.02 \%$. The mean value of demarcated opacities in the first permanent molars was 3.32. Of the 142 children with MIH $48.6 \%$ had lesions only in molars and $51.4 \%$ had both, molars and incisor affected. The most common type of defect was demarcates opacities $(90.7 \%)$. Tooth with MIH also presented breakdown (5.3\%) and atypical restoration (4.0\%). No teeth had been extracted due MIH. Overall caries experience was 0.48 (sd 1.04) for permanent teeth and 1.06 (sd 1.81) for deciduous teeth. A positive correlation was found between MIH $(\mathrm{DMFT}=$ 0.89 ; sd 1.18) and caries experience in permanent teeth $(\mathrm{p}<$ 0.0001). No correlation was found between the presence of $\mathrm{MIH}$ and the caries $(\mathrm{dmft}=1.24$; sd 1.82) $(\mathrm{p}=0.10)$ or enamel defects $(\mathrm{p}=0.11)$ in the deciduous teeth. MHI was common among Brazilian children and could be considered a marker for increased risk of caries in the studied populations.

Funding: FAPESP (2008/03247-9) and CNPq (47126/2008-7) Brazil.

\section{4 \\ Respiratory Diseases Are Associated with Molar-Incisor-Hypomineralisation}

D. Mach ${ }^{\mathrm{a}, *}$, E. Thiering ${ }^{\mathrm{b}}$, R. Heinrich-Weltzien ${ }^{\mathrm{c}}$, R. Hickel ${ }^{\mathrm{a}}$, J. Heinrich ${ }^{b}, J_{\text {Jühnisch }}{ }^{\mathrm{a}}$

dmach@dent.med.uni-muenchen.de

aDepartment of Conservative Dentistry and Periodontology, Ludwig-Maximilians-University of Munich, b Helmholtz Zentrum of Munich, German Research Centre for Environmental Health, Institute of Epidemiology, 'Department of Preventive and Paediatric Dentistry, Friedrich-Schiller-University of Jena, Germany

The etiology of Molar-Incisor-Hypomineralisation has not yet been clarified and there is insufficient evidence about possible (co)factors which are causing these prevalent demarcated enamel defects. Therefore, our study aimed to associate hypomineralisations in relation to prospectively collected data on potential risk factors, e.g. respiratory diseases, breastfeeding, maternal smoking and parental education. The study consisted of 692 children from the German Infant Nutritional Intervention birth cohort study. The dental examination included the tooth- and surfacerelated detection of caries (WHO standard) and hypomineralisations (criteria by the European Academy of Paediatric Dentistry). Children were categorized into those with Molar-and-IncisorHypomineralisations (M-and- $\mathrm{IH}$ ) and Molar-or-Incisor-Hypomineralisations (M-or-IH). The statistical analysis included the correlation of the prospectively collected health data from the first four years of life with the recorded dental health outcomes at 
the age of 10. The relationship was analyzed using simple and multiple logistic regression analysis. In result, no obvious decay in the permanent dentition (DMF $=0$ ) was found in $79.9 \%$ of the 10 -year-olds. The mean caries experience corresponded to 0.4 (0.9) DMFT and 0.4 (1.1) DMFS. While $30.6 \%$ of all 10 -year-olds were scored with $\mathrm{M}$ or IH the phenomenon of $\mathrm{M}$ and $\mathrm{IH}$ was observed in additional $9.5 \%$ of the study population. The overall mean number of hypomineralisations amounted to 0.9 (1.5) teeth and 1.1 (2.3) surfaces. After adjustment for possible confounding factors, 10-year-olds with at least one episode of respiratory disease within the first four years of life had a 2.23 higher risk (adjusted OR) for the development of $\mathrm{M}$ and IH. This association was found to be statistically significant. In conclusion, early respiratory infections seem to be directly or indirectly related to hypomineralisations. The role of (systemic) medications used for treatment of this condition has to be investigated in future studies.

Funding: The GINI study was funded for 3 years by grants of the Federal Ministry for Education, Science, Research and Technology (Grant No. 01 EE 9401-4), the 6 and 10 years follow-ups of the GINI plus study were partly funded by the Federal Ministry of Environment (IUF, FKZ 20462296). The dental investigation was funded by a grant of the German Research Foundation (Deutsche Forschungsgemeinschaft, FKZ KU 2518/1-1 and HE 3294/7-1). The GABA GmbH, Lörrach, Germany, supported the study with health care packages for all participating children.

\section{5 \\ Influence of MIH on the Need for Restorative Treatment in First Permanent Molars in Children}

C.P. Trindade, V.M. Soviero *

soviero@compuland.com.br

State University of Rio de Janeiro, Brazil

The aim of this study was to identify the proportion of first permanent molars treated due to MIH in a group of newly referred children for treatment at the pediatric dental clinic of the University of the State of Rio de Janeiro, Brazil. The study was approved by the local committee for ethics in research. To be included children should present all four first permanent molars erupted. Diagnosis for molar incisor hypomineralization (MIH) was positive when demarcated opacities or post- eruptive breakdown/atypical restoration was detected in at least one of the first permanent molars, associated or not to affected permanent incisors. The examinations were carried out by two calibrated examiners $(k=0.90$ and $\mathrm{k}=1.0$ for intra-examiner and 0.80 for inter-examiner reliability). From 173 patients considered for the examination, 21 could not be contacted and 26 did not show up for examination. Final sample comprised $126(72.8 \%)$ patients, 7 to 13 years-old, 61 girls and 65 boys. MIH was observed in 43 children (34.1\%). From the total sample, 59 (46.8\%) presented at least one first permanent molar which needed or had received an invasive treatment (restoration or extraction). Among children who needed invasive intervention in first permanent molars, 35/59 (59.3\%) presented MIH. Among those who did not need invasive treatment on these molars, 8/67 (11.9\%) presented MIH. The mean number of first permanent molars with invasive treatment was $1.86( \pm 1.36)$ among children with MIH against $0.64( \pm 1.20)$ among those who had no
MIH. In general, more than $40 \%$ of the first permanent molars which were treated by an invasive intervention presented enamel defect. The presence of MIH increased significantly the number of first permanent molars treated by an invasive intervention.

\section{6 \\ Eating Habits and Dental Caries Experience among Students of Strasbourg University (France)}

\section{Bigeard *, D. Muller, M. Jehl, L. Brisset, A.M. Musset}

bigeardl@msn.com

Dental Faculty, Strasbourg, France

Objectives: To identify eating habits and dental health indicators of young French adults. Design: Transversal descriptive study. Complete medical and dental evaluation. Individual interview to assess self-perception of health, oral hygiene and dietary habits. Subjects: The 203 students aged 18 to 25 years (mean 20.9), $70 \%$ girls, called for annual check-up by the Strasbourg University Health Center, October to December 2007. Results: A significant difference $(\mathrm{p}<0.05)$ appeared between good health assessed by medical records (87.6\%) and self-perception of good health (70.7\%). More boys than girls (76.2 vs. $23.8 \%$ ) had self-perception of good health $(\mathrm{p}<0.05)$. The mean BMI $(21.7$, s.d. $=2.99)$ was comparable to that of the 18-25 French population $(21.5$ s.d. = 3.4; Obépi, 2006). $75.6 \%$ took breakfast daily and $93.0 \%$ lunch and dinner. $13.6 \%$ drunk soft drinks every day, $2.5 \%$ ate sweets more than twice a day, with no significant difference between boys and girls. $66.0 \%$ declared to brush their teeth twice a day with no significant sex related difference. $29.9 \%$ were caries free; mean DMFT was 3.20 (s.d. = 3.52), DMFS 4.80 (s.d. =6.61). The SIC was 7.21 (s.d. $=0.45$ ). Among the $8.9 \%$ of overweight and obese (BMI $\geq 25.00$ ) only $10.5 \%$ were caries free while they were significantly more (32.2\%) in the underweight and normal group $(\mathrm{BMI}<24.99)(\mathrm{p}<0.05)$. Conclusion: The meal pattern of the students remained very close to the traditional French model. Most of them showed good general and dental health, having healthy eating and oral hygiene habits. One third of the group cumulated bad eating and poor dental health. These results confirmed the need to identify the risk groups among young adult's population to focus health prevention measures.

\section{7 \\ Acidogenicity of Two Different Types of Bread \\ S. Mortazavi *, S. Noin \\ sh_mortazavi@dnt.mui.ac.ir \\ Dental School, Dental Research Centre, Isfahan University of Medical Sciences, Iran}

Aim: To compare dental plaque $\mathrm{pH}$ following consumption of two types of bread with different cooking process and physical characteristics. Methods: Using a $\mathrm{pH}$-microelectrode the inter proximal plaque $\mathrm{pH}$ was measured in 10 healthy adults following consumption of two types of bread as well as sucrose solution. The study was approved by IUMS Ethic Committee. All the partici- 
pants had signed a written consent form. The volunteers' average DMFT was 6.101 .56 , saliva streptococcus mutans colony count was $>105$. Saliva buffering capacity and flow rate were in normal ranges. Plain traditionally baked 'Sangak' and soft bulky 'Baguette' and \%10 sucrose solution were tested. For each subject baseline plaque $\mathrm{pH}$ was recorded and followed by 1/5/10/15/20/30 min intervals. Mean $\mathrm{pH}$ for test products were analyzed by ANOVA for either jaw. Tukey test was performed to compare groups. Results: Since all subjects were 'tooth brushers' and plaque was younger than $72 \mathrm{~h} \mathrm{pH}$ with non of samples dropped to lower than 6.Sucrose solution caused the most pronounced $\mathrm{pH}$ drop, from $7.15 \pm 0.33$ at baseline to $6.78 \pm 0.29$. Mean plaque $\mathrm{pH}$ of $10 \%$ sucrose solution and Baguette were not statistically different at $1 / 20 / 30$ min ( $p>0.05$ ); however; mean plaque $\mathrm{pH}$ of Sangak and Baguette showed significant differences at $0 / 1 / 20 / 30 \mathrm{~min}(\mathrm{p}<$ $0.05)$. Sucrose solution caused dramatic plaque $\mathrm{pH}$ drop during first $10 \mathrm{~min}$ and then within $30 \mathrm{~min}$ returned to baseline $\mathrm{pH}$. For two bread samples within first $10 \mathrm{~min} \mathrm{pH}$ increased and then started to decrease during tenth to fifteenth min. Conclusion: In this study mean $\mathrm{pH}$ of Sangak was higher than Baguette in all recorded times. Differences could be contributed to dissimilarities in consistency, carbohydrate content and rate of starch gelatination between two bread types.

Supported by Isfahan Dental Research Centre, IUMS.

\section{8 \\ Fluorosis Risk in Populations with Different Levels of Fluoride Exposure}

M.G. McGrady ${ }^{\text {a, }}{ }^{*}$, R.P. Ellwood ${ }^{\text {a }}$, P. Srisilapanan ${ }^{b}$, N. Korwanich $^{\text {b }}$, I.A. Pretty ${ }^{\mathrm{a}}$

michael.mcgrady@manchester.ac.uk

aSchool of Dentistry, University of Manchester, UK; ${ }^{\mathrm{b}}$ Faculty of

Dentistry, Chiang Mai University, Thailand

At low or moderate fluoride exposure fluorosis may present as diffuse white lines or opacities of the enamel surface. However, exposure to higher levels of fluoride may result in more severe hypomineralization with pitting and loss of the surface enamel. A population exposed to high levels of fluoride in groundwater exists in Chiang Mai, Thailand where individuals are advised to consume low fluoride drinking water but cooking water fluoride levels may still present a risk to fluorosis. This study was an observational cross-sectional survey in populations with varying exposures to fluoride from cooking and drinking water. Consented subjects were examined and standardised $35 \mathrm{~mm}$ images were taken of clean, dried maxillary central incisors. The images were viewed in a randomised, blind manner by two examiners providing a consensus score for fluorosis using the Thylstrup and Fejerskov (TF) Index. In total 560 subjects were included in the analysis. Cross-tabulations for fluorosis score and water fluoride content demonstrated a clear increase in TF score with increasing fluoride content for both drinking and cooking water. Regression analysis using categorical variables created from the water data demonstrated that the odds ratio of developing significant fluorosis $(\mathrm{TF}>3)$ in subjects consuming low fluoride $(<0.9 \mathrm{ppm})$ drinking water and high fluoride $(>0.9 \mathrm{ppm})$ cooking water was
4.7 (95\% CI 2.1 to 10.8 ) relative to subjects consuming low fluoride cooking and drinking water. Subjects consuming high fluoride drinking and cooking water had an odds ratio for the risk of significant fluorosis of 11.4 (95\% CI 4.8 to 27.1). The study suggests that consuming high fluoride drinking and cooking water results in an increased risk of developing fluorosis and that cooking water fluoride must be considered in risk assessment.

Funding: Dr. Pretty is funded by a Clinician Scientist Award from the National Institute for Health Research.

Supported by Colgate Palmolive Dental Health Unit, Manchester, UK.

\section{9 \\ Effect of Oscillating Fluoride Doses on Fluorosis Development in Rats}

D.B. Catani ${ }^{\mathrm{a}, *}{ }^{\text {, L.M.A. } \text { Tenuta }^{\mathrm{a}} \text {, F.A. Andaló }}{ }^{\mathrm{b}}$, J.A. Cury ${ }^{\mathrm{a}}$

danilocatani@yahoo.com.br

aPiracicaba Dental School, UNICAMP, Piracicaba, and

${ }^{b}$ Institute of Computing, UNICAMP, Campinas, Brazil

The effect of fluoride (F) on animals exposed to constant chronic F doses is well known, but there is no information about the effect of chronic oscillating doses, to which human are commonly exposed, what was evaluated in the present study. The study was approved by the Animal Ethics Committee of UNICAMP. Twenty 35-days-old Wistar female rats received water with constant F concentration at $0,12.5,25$ or $37.5 \mu \mathrm{g} \mathrm{F} / \mathrm{ml}$ or oscillating at every $72 \mathrm{~h}$ between 12.5 and $37.5 \mu \mathrm{g} \mathrm{F} / \mathrm{ml}$ (mean exposure equals to $25 \mu \mathrm{g} \mathrm{F} / \mathrm{ml}$ ). After 78 days, animals were sacrificed, and $\mathrm{F}$ concentrations in plasma and femur were determined. Dental fluorosis score in the rat incisor was estimated by an image analysis method based on the intensity of the pigmented banding on the surface of incisors. Fluorosis scores were (mean $\pm \mathrm{SD}, \mathrm{n}=$ 4) $1.1 \pm 0.3,1.7 \pm 0.6,2.9 \pm 0.7,4.1 \pm 0.9$ and $2.8 \pm 0.7$ pixel values respectively for groups $0,12.5,25,37.5$ and oscillating. $F$ concentrations in plasma were $2.1 \pm 0.3,4.3 \pm 0.5,5.5 \pm 1.7,7.5$ \pm 2.4 and $5.9 \pm 0.8 \mu \mathrm{M}$, and in the bone, $249.2 \pm 91.5,714.9 \pm$ 41.8, 1,016.9 $\pm 105.7,1,555.7 \pm 112.2$ and $1,082.7 \pm 108.6 \mu \mathrm{g} \mathrm{F} / \mathrm{g}$ dry weight, respectively. A significant linear increase in the variables values was observed according to the increase in F concentration in the water for the constant groups $(p<0.0001)$. No difference between the group which received oscillating doses and the group receiving $25 \mu \mathrm{g} \mathrm{F} / \mathrm{ml}$ was observed (ANOVA, $\mathrm{p}>0.05$ ). The results suggest that the biological effect of $\mathrm{F}$ on animals chronically exposed to symmetrical oscillating $\mathrm{F}$ doses may reflect the effect of the mean of the oscillating doses.

Supported by Capes. 


\section{Caries Research}

\section{Session 9 Erosion}

\author{
170 \\ Effect of Experimental Xylitol and \\ Fluoride-Containing Toothpastes on Enamel \\ Erosion and Abrasion in vitro \\ A.C. Magalhães *, I.D. Rochel, J.G. Souza, T.C. Silva, A.F.F. Pereira, \\ M.A.R. Buzalaf \\ acm@usp.br \\ Bauru School of Dentistry, University of São Paulo, Brazil
}

This in vitro study aimed to analyze the effect of xylitol and fluoride containing toothpastes to protect against enamel erosion associated or not with abrasion. One hundred bovine enamel specimens were submitted to erosion or erosion plus abrasion for 7 days, testing the following toothpastes: Crest (Procter \& Gamble, NaF, 1,030 ppm F, pH 6.8), 10\% xylitol; 10\% xylitol plus 1,030 ppm F (NaF); 1,030 ppm F (NaF) and placebo (Daudt, Brazil, pH 7.0). All toothpastes present silica as abrasive. The erosive challenges were performed 4 times a day ( 2 min, Coca-Cola, $\mathrm{pH}$ 2.3). The specimens were exposed to the slurries of toothpastes (1:3 water) 2 times daily ( $15 \mathrm{~s}, 0.5 \mathrm{ml}$ ), after the first and the last erosive challenges. Additionally, during the toothpaste treatment, half of the specimens per group were abraded using an electrical toothbrush (Colgate Motions Multi-action, $\mathrm{F}=1.5 \mathrm{~N}$ ). Between the erosive challenges, the specimens were remineralized by artificial saliva ( $\mathrm{pH}$ 6.8). Enamel loss was measured profilometrically ( $\mu \mathrm{m})$. The data were statistically analyzed by two-way ANOVA and Bonferroni's post-hoc test $(\alpha=0.05)$. Crest (3.28 \pm 0.99$)$, 10\% xylitol plus F $(3.46 \pm 1.28)$ and F (3.77 \pm 0.54$)$ toothpastes significantly reduced enamel erosion compared to placebo toothpaste $(5.11 \pm 0.56)$. On the other hand, all toothpastes (3.87-4.92) presented a significant potential to protect against enamel erosion plus abrasion compared to placebo (7.28 \pm 0.91$)$. Only placebo and Crest $(4.72 \pm 0.97)$ toothpastes induced a higher enamel loss when eroded enamel specimens were abraded compared to only eroded specimens. Based on the results, $10 \%$ xylitol plus F toothpaste seems to be a good alternative to reduce enamel loss by erosive-abrasive challenges.

Funding: São Paulo State Research Foundation - FAPESP (Process 2008/11582-2).

\author{
171 \\ Effect of Low-Fluoride Varnish with Supplemented \\ with Trimetaphosphate in an in vitro Erosion Study \\ C.C.R. Martinhon *, M.M. Manareli, A.C.B. Delbem \\ cleidecristina@foa.unesp.br \\ Department of Pediatric Dentistry, Unesp, Universidade \\ Estadual Paulista Araçatuba, SP, Brazil
}

This study aimed to evaluate in vitro action varnishes with high and low fluorides concentration and supplemented with trimetaphosphate about the erosion. Enamel bovine blocks were divided into 12 groups $(n=24)$ in accordance with the number of days of treatment ( 3 or 5 days) and type of varnish used: placebo (without fluorine and TMP), varnish $\mathrm{NaF} 5 \%$, varnish $\mathrm{NaF} 2.5 \%$, varnish NaF 2.5\% TMP 3\%, varnish NaF 2.5\% TMP 5\%, varnish NaF 2.5\% TMP 10\%. The enamel blocks suffered a single application of varnish on the first day of the study. Before starting the study, the varnish was removed and half of the blocks $(n=144)$ were submitted to erosion treatment for 3 days and the other half $(n=144)$ were submitted to erosion treatment for 5 days, with Sprite Zero ${ }^{\circledR}$ for 5 min 4 times a day, respectively. Enamel wear was measured by profilometry. Data were analysed by ANOVA and Cochran tests $(\alpha=0.01)$. Mean wear $( \pm S D, \mu \mathrm{m})$ was significantly reduced for the varnish containing TMP $\left(0.75, \pm 0.10^{\mathrm{b}}\right.$, $0.84 \pm 0.08^{\mathrm{b}}, 0.82 \pm 0.09^{\mathrm{b}}$ and $1.26 \pm 0.20^{\mathrm{d}}, 1.52 \pm 0.24^{\mathrm{d}}, 1.29$ $\pm 0.20^{\mathrm{d}}$, respectively) when compared to varnish $\mathrm{NaF} 5 \%$ and $2.5 \%\left(3.09 \pm 0.51^{\mathrm{a}}, 3.01 \pm 0.55^{\mathrm{a}}\right.$ and $4.29 \pm 0.51^{\mathrm{c}}, 4.78 \pm 0.72^{\mathrm{c}}$, respectively) and placebo varnish (3.09 $\pm 0.49^{\mathrm{a}}, 4.76 \pm 0.52^{\mathrm{c}}$, respectively). The use of a varnish as a vehicle to deliver TMP to enamel was able to prevent wear independent of time of erosive challenge and to open a new possibility for the protection against enamel erosion.

Funding: FAPESP: no. 2009/03869-2. 


\section{2 \\ Fluoride Toothpastes and Special Anti-Erosive Formulations: Effects on Enamel}

L. Neutard* , N. Schlueter, O. Grunau, J. Klimek, C. Ganss

carolina.ganss@dentist.med.uni-giessen.de

Department of Conservative and Preventive Dentistry,

Dental Clinic, Justus-Liebig-University, Giessen, Germany

The type of active agents has been shown to be essential for anti-erosive effects in enamel. The present experiments sought to investigate various conventional and special anti-erosive toothpastes with respect to their anti-erosive properties, and their effects regarding brushing abrasion. For 10 days, enamel samples $(\mathrm{n}=18$ per group) were demineralised (citric acid, $6 \times 2 \mathrm{~min} /$ day; $\mathrm{pH}$ 2.3) and intermittently stored in a mineral salt solution. In Experiment 1 (E1), samples were exposed to toothpaste slurries for $2 \times 2 \mathrm{~min} /$ day. In Experiment 2 (E2), samples were brushed for $15 \mathrm{~s}$ (brushing machine, brushing force $2 \mathrm{~N}$, ADA reference toothbrush) within the 2 min slurry immersion time. The toothpastes were six conventional F-toothpastes (1,450 ppm F as NaF), four special anti-erosive formulations (two F-toothpastes: 1,450 ppm $\mathrm{F}$ as $\mathrm{NaF} / \mathrm{KNO} 3$, and 1,450 ppm $\mathrm{F}$ as $\mathrm{NaF} /$ hydroxyapatite; two F-free toothpastes: zinc-carbonate-hydroxyapatite, and Chitosan), and two Sn-containing products $(3,000$, and 3,500 ppm $\mathrm{Sn})$. Control groups in E1 were erosion only, and a Sn/F mouthrinse, and in E2 brushing with placebo, and immersion in a Sn/F mouthrinse after placebo brushing. Substance loss was quantified profilometrically. In E1, most F-toothpastes and also one F-free formulation reduced substance loss ( $\mathrm{p} \leq 0.05$ each for comparisons to erosion only). The Sn-containing formulations were most effective. In E2, only two F-toothpastes reduced substance loss ( $p \leq 0.01$ each for comparisons to brushing with placebo); the fluoride free preparations had no significant effect. Also one Sncontaining formulation did not reduce substance loss. The rinse after brushing with placebo was more effective than the F-toothpastes. Conventional F-toothpastes reduced the erosive substance loss even under severe erosive conditions, but had limited efficacy regarding brushing abrasion. The special formulations were not superior or even less effective compared to conventional products.

Supported by Justus-Liebig-University, Giessen.

\section{3}

\section{Fluoride Toothpastes and Special Anti-Erosive Formulations: Effects on Dentine}

J. von Hinckeldey*, C. Ganss, V. Fickenscher, J. Klimek, N. Schlueter

carolina.ganss@dentist.med.uni-giessen.de

Department of Conservative and Preventive Dentistry,

Dental Clinic, Justus-Liebig-University, Giessen, Germany

Special toothpastes against erosion have been developed claiming to be superior to conventional F-toothpastes. The present experiments sought to investigate various products with respect to their anti-erosive properties, and their effects regarding brushing abrasion. For 10 days, dentine samples ( $n=18$ per group) were demineralised (citric acid, $6 \times 2 \mathrm{~min} /$ day; $\mathrm{pH} 2.3$ ) and in- termittently stored in a mineral salt solution. In Experiment 1 (E1), samples were exposed to toothpaste slurries for $2 \times 2 \mathrm{~min} /$ day. In Experiment 2 (E2), samples were brushed for $15 \mathrm{~s}$ (brushing machine, brushing force $2 \mathrm{~N}$, ADA reference toothbrush) within the 2 min slurry immersion time. The toothpastes were six conventional F-toothpastes (1,450 ppm $\mathrm{F}$ as $\mathrm{NaF}$ ), four special anti-erosive formulations (two F-toothpastes: 1,450 ppm F as NaF/ KNO3, and 1,450 ppm $\mathrm{F}$ as NaF/hydroxyapatite; two F-free toothpastes: zinc-carbonate-hydroxyapatite, and Chitosan), and two Sn-containing products $(3,000$, and 3,500 ppm Sn). Control groups in E1 were erosion only, and a $\mathrm{Sn} / \mathrm{F}$ mouthrinse, and in E2 brushing with placebo, and immersion in a Sn/F mouthrinse after placebo brushing. Substance loss was quantified profilometrically after enzymic removal of organic surface material. In both experiments, all F-containing slurries reduced substance loss (E1: $\mathrm{p} \leq 0.001$ each for comparisons to erosion only, E2: $\mathrm{p} \leq 0.01$ each for comparisons to brushing with placebo), while the F-free products exhibited no significant effect. Significant differences between F-toothpastes were observed. In E1, the application of the rinse was significantly more effective than F-toothpastes, but in minor order. The application of the rinse after brushing with placebo (E2) showed a similar effect than most F-toothpastes. Conventional F-toothpastes reduced the erosive and erosive/abrasive substance loss even under severe erosive conditions. The special formulations were not superior or even less effective compared to conventional products.

Supported by Justus-Liebig-University Giessen.

\section{4 \\ Prevention of Dental Erosion-Abrasion Development by Mouthrinses}

\author{
A.T. Hara *, F. Lippert, D.T. Zero \\ ahara@iupui.edu \\ Indiana University School of Dentistry, USA
}

Anti-erosion mouthrinses have been developed and introduced to the market. This study investigated whether 3 mouthrinses (ES: 100ppm F/NaF, pH3.25; EL: 500ppm F/AmF $+\mathrm{SnCl}_{2}$, pH4.16; PR: 450 ppm F/NaF, pH6.26; DW: deionized water, as negative control) were able to prevent dental loss in an in vitro erosion-brushing abrasion model. They were tested in 2 conditions: brushing with fluoride-containing (FD) or fluoride-free placebo (PD) dentifrices, generating 8 experimental groups $(\mathrm{n}=$ $8)$. Sixty-four specimens containing slabs $\left(4 \times 4 \mathrm{~mm}^{2}\right)$ of human enamel and root dentin were prepared, flattened and polished. Their surfaces were protected, exposing a central area of $1 \times 4$ $\mathrm{mm}^{2}$. Specimens were demineralized for $2 \mathrm{~min}$ in $1 \%$ citric acid (pH 3.8), immersed in artificial saliva for $60 \mathrm{~min}$ and brushed for 200 strokes, in automated brushing machine ( $\sim 200 \mathrm{~g}$ load) with one of the testing dentifrices. Subsequently, the specimens were immersed in the mouthrinses for $1 \mathrm{~min}$. The erosion-reminbrushing-mouthrinse treatment cycle was repeated $3 \times /$ day, for 3 days. Enamel and dentin surface losses were measured by optical profilometry and independently analyzed by two-way ANOVA, followed by Tukey tests $(\alpha=0.05)$. For enamel, the interaction mouthrinse $x$ dentifrice was significant $(\mathrm{p}<0.001)$. When asso- 
ciated to $\mathrm{PD}$, the enamel loss (standard-deviation), in $\mu \mathrm{m}$, was: EL: $-1.42(0.19)^{\mathrm{a}}$, PR: $-2.40(0.59)^{\mathrm{b}}$, ES: $-2.84(0.53)^{\mathrm{bc}}$ and DW: $-3.40(0.58)^{\mathrm{c}}$; while when associated to FD: EL: $-1.21(0.29)^{\mathrm{a}}$, PR: $-1.93(0.42)^{\mathrm{b}}$, ES: $-2.39(0.44)^{\mathrm{b}}$ and DW: $-1.83(0.30)^{\mathrm{b}}$ (different superscript letters represent significant differences). Fluoride dentifrice significantly reduced enamel loss when associated to all mouthrinses, except to EL $(\mathrm{p}=0.350)$. For dentin, there was no significant interaction $(\mathrm{p}=0.797)$. Brushing with FD caused reduced dentin loss $(\mathrm{p}=0.004)$. No protection was provided by any mouthrinse, with ES increasing dentin loss $(\mathrm{p}=0.09)$. Mouthrinses presented different anti-erosion-abrasion potential for enamel, which seemed irrelevant for PR and ES when brushing with FD. For dentin, the mouthrinses showed to be either not beneficial or detrimental.

\section{5 \\ In vitro Evaluation of Different Fluoride Treatments on Hydrochloric Acid-Induced Erosion

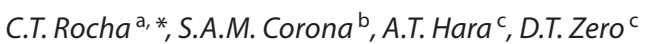 \\ ahara@iupui.edu \\ ${ }^{\mathrm{a}}$ Department of Paediatric, Preventive and Social Dentistry, and \\ ${ }^{b}$ Department of Restorative Dentistry, Ribeirão Preto School of Dentistry, University of São Paulo, Brazil; 'Oral Health Research Institute, Indiana University School of Dentistry, Indianapolis, USA}

This study evaluated the erosion protective effect of different fluoride treatments. 112 bovine enamel specimens $(5 \times 5 \mathrm{~mm})$ were prepared, flattened and polished. Adhesive tapes were placed on the surface, leaving an area of $5 \times 1 \mathrm{~mm}$ exposed. Specimens were immersed for $2 \mathrm{~min}$ in $0.01 \mathrm{M} \mathrm{HCl}(\mathrm{pH} 2.15)$, rinsed with deionized water and immersed in artificial saliva (120 min), $4 \times /$ day. After the 1st day, an area of $2 \times 1 \mathrm{~mm}$ (covering both exposed and tape-covered areas) was analyzed by optical profilometry. The surface loss was calculated and specimens were assigned into 8 groups $(\mathrm{n}=14)$ using balanced randomization: Control (no treatment), $1.23 \%$ APF gel, $2.0 \% \mathrm{NaF}$ gel, $0.4 \% \mathrm{SnF}_{2}$ gel, $1,100 \mathrm{ppm}$ F toothpaste (Crest Cavity Protection ${ }^{\circledR}$ ), 1,450 ppm F toothpaste $\left(\right.$ Colgate Total ${ }^{\circledR}$ ), 5,000 ppm F toothpaste (Prevident ${ }^{\circledR}$ ) and $5 \%$ $\mathrm{NaF}$ varnish. A single topical application of varnish and of each gel (for $1 \mathrm{~min}$ ) was performed on the 2 nd day. Fresh slurries of the testing toothpastes were applied for $2 \mathrm{~min}, 2 \times /$ day, from the 2 nd to the 6th cycling day. Surface loss was measured, after the 3rd and 6 th cycling days. After the $3 \mathrm{rd}$ day, $\mathrm{SnF}_{2}$ showed the lowest surface loss ( $\mathrm{p}<0.001)$. APF, Prevident, $\mathrm{NaF}$ and Colgate were not significantly different from Control ( $p>0.05)$. After the 6th day, $\mathrm{SnF}_{2}$ and varnish showed the lowest surface loss $(\mathrm{p}<0.001)$, but these were not significantly different from Colgate, APF and Crest ( $p>0.05)$. Prevident and $\mathrm{NaF}$ showed the highest surface loss, similar to the control ( $p>0.05$ ). The results of this study suggest that a single application of $\mathrm{SnF}_{2}$ gel and APF gel may be as effective as the daily exposure to fluoride dentifrice in the short period investigated.

\section{6 \\ Influence of CPP-ACP and Fluoride on Surface Rehardening of Eroded Enamel}

T.T. Tauböck* , A. Stillhard, T. Attin, F.J. Wegehaupt

tobias.tauboeck@zzmk.uzh.ch

Clinic for Preventive Dentistry, Periodontology and Cariology,

University of Zurich, Switzerland

This in situ study aimed to investigate the influence of intraoral and extraoral application of both casein phosphopeptideamorphous calcium phosphate (CPP-ACP) paste and fluoride containing mouthrinse on surface rehardening of acid softened enamel. Ten volunteers performed five experimental run, each. In each run, four bovine enamel specimens were extraorally softened in an acidic beverage (Sprite light) for $2 \mathrm{~min}$ and subsequently worn intraorally for $5 \mathrm{~min}$, using individual palatal appliances. Thereafter, specimens were treated with CPP-ACP paste (Tooth Mousse, GC, Japan) or $250 \mathrm{ppm}$ AmF/SnF2 solution (Meridol, GABA, Switzerland) for 3 min. Application of the test substances was either performed intraorally (run 1 and 2) or after removal of the appliances from the mouth (run 3 and 4). Untreated specimens served as control (run 5). After application, specimens were exposed to the oral cavity for further 3:55 h. Knoop microhardness (KHN) of the specimens was measured at baseline, immediately after softening and at the end of each run. Data were statistically analyzed by one-way ANOVA and Bonferroni/Dunn posthoc test. Baseline KHN (mean \pm SD) amounted to $339.3 \pm 15.7$. Immediately after softening, mean KHN was reduced to $255.7 \pm$ 25.0. Significant rehardening after intraoral exposure was observed for all groups, but did not achieve baseline values. Extraoral application of Tooth Mousse resulted in significantly higher KHN (290.2 \pm 31.4$)$ compared to extraoral application of Meridol $(268.3 \pm 25.9)$ and untreated controls $(270.7 \pm 29.0)$. KHN after intraoral application of both Tooth Mousse $(277.0 \pm 27.4)$ and Meridol (284.2 \pm 28.6$)$ was not significantly different to untreated controls. It is concluded that intraoral application of both CPPACP paste and $250 \mathrm{ppm} \mathrm{AmF/SnF2} \mathrm{solution} \mathrm{provides} \mathrm{no} \mathrm{benefit}$ regarding surface rehardening of previously acid softened enamel.

\section{7 \\ Effect of a CPP-ACP Paste on Erosive Tooth Wear in vitro}

U. Schiffner *, W. Hoßdorf

schiffner@uke.uni-hamburg.de

University of Hamburg, Dental School, Germany

A CPP-ACP containing paste is used by patients at high risk for dental erosion. The aim of the study was to evaluate the erosion inhibiting effect of the paste and to compare it with the effect of a single fluoride treatment. 75 enamel-dentine-specimens were prepared and allocated to a control and two test groups. All specimens were subjected to 5 erosive challenges daily by immersing them into $0.1 \%$ citric acid for $10 \mathrm{~min}$. The specimens of one test group were fluoridated with Elmex Gel once prior to the first ero- 
sive challenge, while the remaining specimens were covered twice daily with a thin layer of the CPP-ACP containing MI Paste Plus. All specimens were stored in artificial saliva. After 7 days the specimens were ground to thin sections. The depths of the erosions were determined microscopically and statistically compared. The results showed in enamel an erosive loss of $173 \pm 24$ $\mu \mathrm{m}$ (control), $137 \pm 24 \mu \mathrm{m}$ (fluoride gel) and $121 \pm 25 \mu \mathrm{m}$ (CPP group). In dentine, the resp. loss was $51 \pm 17 \mu \mathrm{m}$ (control), $34 \pm$ $7 \mu \mathrm{m}$ (fluoride gel) and $28 \pm 8 \mu \mathrm{m}$ (CPP). The depths of the demineralised areas were $219 \pm 34 \mu \mathrm{m}, 167 \pm 28 \mu \mathrm{m}$, and $156 \pm$ $24 \mu \mathrm{m}$, resp., in enamel, and $263 \pm 31 \mu \mathrm{m}, 208 \pm 27 \mu \mathrm{m}$, and 193 $\pm 20 \mu \mathrm{m}$ in dentine. Statistical analysis revealed an erosion inhibiting effect of both the fluoride and the CCP treatment, the latter showing significantly better results than the fluoride regimen (Wilcoxon test). The study indicates that erosive wear can be reduced by covering tooth surfaces regularly with a CPP-ACP containing paste, and that the reduction is slightly higher than after a single fluoride gel treatment.

\section{8 \\ Effect of Metals Ions on Demineralization of Bovine Enamel Powder by Coca-Cola}

C.M. Souza-e-Silva *, H.A.B.S. Pereira, F.M. Italiani, A.L. Leite, M.T. Kato, M.A.R. Buzalaf

mbuzalaf@fob.usp.br

Bauru Dental School, University of São Paulo, Bauru, Brazil

This study evaluated the effect of metallic ions supplemented to Coca-Cola on its erosive potential. At time zero, the beverage was added to powdered enamel (1 mg enamel powder/10 ml beverage) and shaken for $30 \mathrm{~s}$. The sample (control) was immediately centrifuged for $30 \mathrm{~s}(11,000 \mathrm{rpm})$ and the supernatant was removed at $1 \mathrm{~min} 40 \mathrm{~s}$. This procedure was repeated with the beverage containing different concentrations $(1.25,2.5,5,10,15,30$ and $60 \mathrm{mmol} / \mathrm{l}$ ) of $\mathrm{MnSO}_{4} \cdot \mathrm{H}_{2} \mathrm{O}, \mathrm{MgSO}_{4} \cdot 7 \mathrm{H}_{2} \mathrm{O}, \mathrm{CuSO}_{4} \cdot 5 \mathrm{H}_{2} \mathrm{O}$ or $\mathrm{ZnSO}_{4} \cdot 7 \mathrm{H}_{2} \mathrm{O}$. The combination of different ions at 1.0 or 10 $\mathrm{mmol} / \mathrm{l}$, including iron, was also tested. The phosphate and calcium released in the medium were analyzed spectrophotometrically. Data were analyzed using ANOVA and Tukey's test $(\mathrm{p}<$ 0.05). The addition of $\mathrm{Mn}^{2+}$ to Coca-Cola, in concentrations above $2.5 \mathrm{mmol} / \mathrm{l}$, caused a slight reduction on enamel dissolution, from 3 to $7 \%$, although the differences were not significant when compared to the control. The addition of $\mathrm{Cu}^{2+}$ to CocaCola concentrations up to $5 \mathrm{mmol} / \mathrm{l}$ led to an increase in the dissolution of powdered enamel. However, in higher concentrations, there was a small reduction on enamel dissolution, although this was not significantly different in relation to control. When $\mathrm{Mg}^{2+}$ was added to Coca-Cola there was no alteration in the dissolution of powdered enamel. Regarding the addition of $\mathrm{Zn}^{2+}$, at concentrations equal to $10 \mathrm{mmol} / \mathrm{l}$ or higher, there was a significant reduction in the dissolution of powdered bovine enamel in relation to the control $(12,10,13$ and $20 \%$, respectively for concentrations of $10,15,30$ and $60 \mathrm{mmol} / \mathrm{l})$. The best protective effect $(27 \%$ reduction) was observed for the combination of the different metallic ions. The results suggest that combination of metallic ions can be an alternative to reduce the erosive potential of Coca-Cola.

Funding: FAPESP (Grant n. 2007/04222-7).

\section{9 \\ Screening of $\mathrm{CO}_{2}$ Laser (10.6 $\left.\mu \mathrm{m}\right)$ Parameters for Prevention of Enamel Erosion}

\author{
M. Esteves-Oliveira ${ }^{\mathrm{a}, *},{ }^{*}$. Yu ${ }^{\mathrm{b}}$, F. Lampert ${ }^{\mathrm{a}}$, C.P. Eduardo ${ }^{\mathrm{c}}$, \\ T. Attin $^{\mathrm{b}}{ }^{\mathrm{b}}$ A. Wiegand ${ }^{\mathrm{b}}$ \\ mestevesoliveira@ukaachen.de \\ ${ }^{a}$ Clinic of Conservative Dentistry, Periodontology and \\ Preventive Dentistry, RWTH Aachen University, Germany; \\ ${ }^{b}$ Clinic for Preventive Dentistry, Periodontology and Cariology,

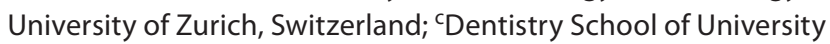 \\ of Sao Paulo, Brazil
}

As $\mathrm{CO}_{2}$ laser irradiation is known to cause decrease of enamel demineralization in caries models and the same has not yet been observed for erosion, the aim of this study was to find $\mathrm{CO}_{2}$ laser $(10.6 \mu \mathrm{m})$ parameters to increase enamel resistance to erosive acid attack. Different laser parameters were tested in twelve groups (each $\mathrm{n}=10$ enamel samples) with fluencies varying from 0.1 to $0.9 \mathrm{~J} / \mathrm{cm}^{2}$, pulse durations from 80 to $400 \mu \mathrm{s}$ and repetition rates from 180 to $700 \mathrm{~Hz}$. Non-lased samples served as control. Lased and control samples were eroded by exposure to hydrochloric acid $(\mathrm{pH} 2.6)$ in a small erosion chamber under continuous acid flow conditions $(60 \mu \mathrm{l} / \mathrm{min})$. Calcium and phosphate release into the acid was monitored colorimetrically at $1 \mathrm{~min}$ intervals up to a total erosion time of $15 \mathrm{~min}$. Calcium and phosphate release was calculated as percentage of the mean calcium and phosphate release of the respective controls and statistically analysed by oneway ANOVA $(p<0.05)$ at each time point. Calcium and phosphate release was significantly reduced in groups irradiated at 0.4 $\mathrm{J} / \mathrm{cm}^{2}(200 \mu \mathrm{s})$ with 400 and $450 \mathrm{~Hz}$ and $0.6 \mathrm{~J} / \mathrm{cm}^{2}$ (300 $\left.\mu \mathrm{s}\right)$ with 200, 250 and $300 \mathrm{~Hz}$. Thereby, calcium and phosphate losses were reduced by only $10-15 \%$ compared to control, and these effects were restricted to up to $10 \mathrm{~min}$ acid flow. In all other groups, enamel erosion could not be reduced significantly. The $\mathrm{CO}_{2}$ laser parameters tested in the present study showed only limited prevention of enamel erosion. Thus, further research is necessary to determine laser parameters with a higher protective capability.

\section{0 \\ Preventive Effect of Metalloproteinase Inhibitors on the Degradation of the Dentin Organic Matrix in vitro}

M.A.R. Buzalaf*, M.T. Kato, A.L. Leite, A.R. Hannas

mbuzalaf@fob.usp.br

Bauru Dental School, University of São Paulo, Bauru, Brazil

The degradation of organic material in dentin by matrix metalloproteinases (MMPs) is expected to increase the wear of erosive lesions. Green tea polyphenols (especially epigallocatechin 3-galatte (EGCG)), chlorexidine (CHX) and ferrous sulphate $\left(\mathrm{FeSO}_{4}\right)$ have been reported to inhibit MMP-2 and MMP-9 activities. Thus, the aim of this study was to analyse the effect of MMP inhibitors on collagen degradation and wear of demineralised dentin. Demineralisation of the dentin slices ( $n=45 /$ group) was performed in a $1.0 \times 0.5 \mathrm{~cm}$ window with $0.87 \mathrm{M}$ citric acid, 
$\mathrm{pH} 2.3$, for $36 \mathrm{~h}$. In sequence, specimens were or not (NT) treated with gels containing EGCG $(400 \mu \mathrm{M}), \mathrm{CHX}(0.012 \%), \mathrm{FeSO} 4$ (1 $\mathrm{mM}), \mathrm{NaF}(1.23 \%)$ or no active compound (P, placebo). Gels were applied on dentin only once in a thin layer and removed after $1 \mathrm{~min}$. Specimens were stored in artificial saliva $\left(5\right.$ days, $\left.37^{\circ} \mathrm{C}\right)$ containing EDTA-free protease inhibitors with the addition of collagenase (Clostridium hystoliticum, $200 \mathrm{U} / \mathrm{ml}$ ). Collagen degradation was analysed by hydroxyproline content $(\mu \mathrm{g} / \mathrm{ml})$ in the incubation solutions (after pooling 9 samples per group) and the dentin wear was evaluated in 12 specimens/group by profilometry. Data were analysed by ANOVA and Tukey's test $(\mathrm{p}<0.05)$. There was a significant lower hydroxyproline concentration $( \pm S D, \mu g / m l, n=5)$ in artificial saliva derived from treatment with gels containing EGCG $\left(0.33 \pm 0.05^{\mathrm{b}}\right)$, CHX $\left(0.32 \pm 0.10^{\mathrm{b}}\right)$ and $\mathrm{FeSO}_{4}\left(0.34 \pm 0.06^{\mathrm{b}}\right)$ when compared with $\mathrm{NaF}(0.56 \pm$ $\left.0.02^{\mathrm{c}}\right), \mathrm{P}\left(0.96 \pm 0.02^{\mathrm{a}}\right)$ or NT $\left(0.94 \pm 0.05^{\mathrm{a}}\right)$. Similarly, the mean wear $( \pm S D, \mu \mathrm{m})$ was significantly reduced for the gels containing MMPs inhibitors $\left(85.9 \pm 26.5^{\mathrm{b}}, 92.01 \pm 18.19^{\mathrm{b}}\right.$ and $85.9 \pm 23.6^{\mathrm{b}}$ for, EGCG, $\mathrm{CHX}$ and $\mathrm{FeSO}_{4}$, respectively) when compared to $\mathrm{NaF}$ $\left(131.35 \pm 33.56^{\mathrm{c}}\right), \mathrm{P}\left(170.83 \pm 45.19^{\mathrm{a}}\right)$ or NT $\left(180.98 \pm 36.78^{\mathrm{a}}\right)$. The use of a gel as a vehicle to deliver MMP inhibitors to dentin was shown to consistently reduce collagen degradation and wear.

Financial support: FAPESP (Grants 07/08389-3 and 07/04209-0).

\section{1 \\ Impact of Tannic Acid, a Cross-Linking Agent, and Chlorhexidine, a Matrix Metalloproteinase Inhibitor, on Dentin Wear}

M.T. Kato ${ }^{a}{ }^{*}$, A.R. Hannas a, J.C. Pereira ${ }^{\text {a }}$, A.C. Magalhães ${ }^{\text {a }}$, L. Tjäderhane ${ }^{b}$, M.A.R. Buzalaf ${ }^{a}$

mbuzalaf@fob.usp.br

aBauru Dental School, University of São Paulo, Bauru, Brazil;

bUniversity of Oulu, Finland

Collagen type I is the major organic component of dentine and its stability may have a great impact on the degradation of the organic matrix. This study aimed to analyse the impact of tannic acid (TA), a collagen cross-linking agent, and chlorhexidine (CHX), a matrix metalloproteinase inhibitor, on dentine wear. The hypothesis tested was that the pre-treatment with 20\% TA, $0.12 \% \mathrm{CHX}$ or the combination of both would influence enzymatic degradation of demineralised dentine matrix. Bovine dentine blocks $(\mathrm{n}=55)$ were demineralised in $10 \%$ phosphoric acid for $5 \mathrm{~h}$. Nail varnish was applied on both sides of the surface of each sample to maintain a reference surface for wear determination. Samples $(\mathrm{n}=55)$ were randomly divided into 5 treatment groups: negative control (distilled water, $\mathrm{pH}$ 6.0), positive control $(1,100$ ppm F, as NaF), 20\% TA, 20\% TA $+0.12 \% \mathrm{CHX}+0.12 \%$ CHX. TA and CHX were dissolved in distilled water. Specimens were kept in the treatment solutions for $1 \mathrm{~h}$, thoroughly rinsed, and then subjected to enzymatic challenge for $24 \mathrm{~h}$ with bacterial collagenase (Clostridium hystoliticum, $100 \mathrm{U} / \mathrm{ml}$ ) in an ammonium bicarbonate buffer $\mathrm{pH}$ 9.5. Dentine wear was assessed by profilometry. Data were analysed by ANOVA and Tukey's test ( $\mathrm{p}<$ $0.05)$. The mean wear $( \pm S D, \mu \mathrm{m})$ found for $\mathrm{NaF}(2.28 \pm 0.56)$ was not significantly different from control (2.24 \pm 0.48$)$. Both TA $(1.66 \pm 0.60)$ and $\mathrm{CHX}(1.52 \pm 0.43)$ were able to significantly reduce the wear, but no synergistic effect was detected for their combination $(1.54 \pm 0.34)$. Thus, dentine treatment with TA, $\mathrm{CHX}$ or their combination decreased collagenase digestion of the organic matrix and might be useful to reduce wear.

Funding: CNPq (Grant 557863/2008-2) and FAPESP (Grant 2007/04209-0).

\section{2 \\ A Luminescent Assay Approach for the Assessment of Erosive Challenge

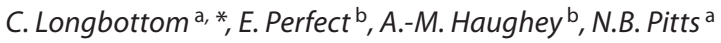 \\ c.longbottom@cpse.dundee.ac.uk \\ aUniversity of Dundee, ${ }^{\text {b }}$ Lux DS, Edinburgh, UK}

Aim: To determine the sensitivity, for 3 different erosive attack durations, of a method which uses a luminescent calcium-ion marker (WO2008075081 GB2445460 (A)) to capture released calcium ions. Methods: 6 extracted human teeth, hemisected buccolingually to create matched sample pairs, each had nail varnish applied to create an enamel 'window'. The samples were rinsed in de-ionised water before incubation for $2 \mathrm{~h}$ at $37^{\circ} \mathrm{C}$ in a humid environment. Two sets of one of each sample pair were challenged for either 1, 3 or $60 \mathrm{~min}$ in $3 \mathrm{ml} 50 \mathrm{~mm}$ phosphoric acid ( $\mathrm{pH} \mathrm{1.9)}$ ), with the matching sample pair incubated for the same amount of time in neutralised acid (with $1 \mathrm{M}$ sodium hydroxide) ( $\mathrm{pH}$ 6.9). After challenge, the sample was removed from solution and dipped in de-ionised water. An image of the sample was taken in the light ( $40 \mathrm{~ms}$ capture) and then in darkness (60 s capture) after application of the luminescent calcium marker. Light output was measured by determining grey-scale values with an image analysis programme. The solutions used for the challenge were also tested with the luminescent marker. Results: Results show that: (a) using the image grey-scale values, there was a trend of increased light output as the erosive challenge increased in time and (b) for each sample, enamel challenged with neutral solution produced at least 50 million Relative Light Units (RLUs) less light than that treated with acid produced, which was between $60-$ $>130$ million RLUs. Conclusions: The developed luminescent calcium-ion marker assay method was capable of detecting calcium ions released after 1 minute of acid-erosive challenge and appeared sensitive to an increased time of attack of up to $1 \mathrm{~h}$.

Supported by a KTP Programme grant no. 6480 and a TSB grant Project No: TP11/TFH/6/1/AG240J.

\section{3 \\ Effects of Modelling Erosive Conditions on the Outcome of Erosion-Abrasion Studies}

\section{N. Schlueter*, J. von Hinckeldey, L. Neutard, J. Klimek, C. Ganss \\ nadine.schlueter@dentist.med.uni-giessen.de \\ Department of Conservative and Preventive Dentistry, \\ Dental Clinic, Justus-Liebig-University, Giessen, Germany}

There is no standard for testing anti-erosive/anti-abrasive agents, making the assessment of study results difficult. One factor in experimental modelling is the erosive medium, the impact 
of which on study outcome was investigated. In four experiments, enamel specimens were demineralised (10 days, $6 \times 2 \mathrm{~min} /$ day) using different acids: $1,0.5$, and $0.3 \%$ citric acid at native $\mathrm{pH}(2.3$, 2.8, 2.8 resp.) and $0.3 \%$ citric acid adjusted to $\mathrm{pH}$ 3.6. Each experiment consisted of three groups ( $\mathrm{n}=10$ each). One group served as control (erosion only), in the others, specimens were immersed in slurries of either a 1,400 ppm F-toothpaste or the corresponding placebo $(2 \times 2 \mathrm{~min} /$ day $)$ and brushed during immersion time (15 s, $2 \mathrm{~N}$, ADA reference brush). Between treatments, samples were stored in a mineral salt solution. Tissue loss was determined profilometrically. In control groups, tissue loss $(\mu \mathrm{m}$; mean \pm SD) was $23.7 \pm 9.5,13.5 \pm 4.5,7.5 \pm 2.5$ and $3.3 \pm 3.6$ in experiments with $1 \%, 0.5 \%, 0.3 \%(\mathrm{pH} 2.8)$ and $0.3 \%(\mathrm{pH} 3.6)$ acid, brushing with placebo increased tissue loss by $64,35,46 \%$ (all p $\leq 0.05$ ) and $12 \%$ (n.s.), respectively. Compared to control groups, brushing with F-toothpaste increased tissue loss in the experiment using $1 \%$ citric acid ( $47 \%, \mathrm{p} \leq 0.01)$, but reduced loss by $11 \%$ in the $0.5 \%$ (n.s.) and by $42 \%$ in the $0.3 \%$ (pH 2.8 ) acid experiments $(\mathrm{p} \leq 0.05)$. In the $0.3 \%(\mathrm{pH} 3.6)$ experiment, no substance loss occurred from brushing with F-toothpaste. Instead, deposits with a thickness of $6.9 \pm 8.3$ ( $\mathrm{p} \leq 0.01$ ) were found. The $1 \%$ acid model was more sensible to abrasion effects, while the 0.5 and $0.3 \%$ ( $\mathrm{pH} 2.8$ ) acid model showed both the abrasion effects and the effects of active agents. The $0.3 \%(\mathrm{pH} 3.6)$ acid model was too mild to demonstrate effects of brushing-abrasion.

Supported by GABA International AG, Therwil, Switzerland.

\section{4 \\ Dilution Effect on the Chemical Properties of Acidic Powdered Drinks \\ C.P. Turssi ${ }^{\mathrm{a}}, *$, A.T. Hara ${ }^{\mathrm{b}}$ \\ cecilia.turssi@uniube.br \\ aSchool of Dentistry, University of Uberaba, Brazil; \\ ${ }^{b}$ Oral Health Research Institute, Indiana University, USA}

Some erosive drinks are commercially available in powdered form, and possibilities are that individuals consume them in a more concentrated or dilute form. Consequently, such mixes can have their erosive potential altered. This study aimed to assess the influence of different mixing ratios on the chemical properties of a range of powdered drinks (citrus juice, sports and energy drinks and lemon-flavoured tea). Drinks were prepared regular-concentrated, half-concentrated and double-concentrated and analyzed for $\mathrm{pH}$, titratable acidity, $\mathrm{pH}$-stat and ADAF-ISO screening tests. Drinking water was used as control. The $\mathrm{pH}$-values were determined by a $\mathrm{pH}$ electrode. Titratable acidity was recorded as the volume of $1 \mathrm{~N} \mathrm{NaOH}$ to raise the $\mathrm{pH}$ of the drinks to 7. The $\mathrm{pH}-$ stat test was performed using an automatic titrator in the presence of hydroxyapatite crystal and measured as the volume of $0.1 \mathrm{~N}$ $\mathrm{HCl}$ to maintain the original $\mathrm{pH}$ of the drinks. For the ADAF-ISO test, $\mathrm{pH}$ change of a Ca- $\mathrm{PO}_{4}$ (slightly undersaturated Hap) solution after the addition of prepared drinks was obtained. Calcium concentration $(\mathrm{Ca})$ was analyzed using flame atomic absorption spectrometry, while fluoride (F) amount was measured by a Fspecific electrode. Tests were performed in triplicate in three separate runs. ANOVA and Tukey's test showed that increasing dilu- tion produced: (1) a significant rise in $\mathrm{pH}(\mathrm{p}<0.0001)$; (2) a significant decrease in titratable acidity $(\mathrm{p}<0.0001)$; (3) no effect on the $\mathrm{pH}$-stat test ( $\mathrm{p}>0.3$ ); (4) a significant reduction in the $\mathrm{pH}$ change measured through the ADAF-ISO method ( $\mathrm{p}<0.0001)$; (5) a reduction in $\mathrm{Ca}$ and $\mathrm{F}$ concentration. In conclusion, the results from this study show that the mixing ratio can modulate the majority of the chemical properties of powdered drinks suggesting that their erosive potential may be affected.

\section{5 \\ Quantitative Assessment of Erosion Using Optical Coherence Tomography: A Study of Reference Methods}

H.P. Chew *, C.M. Zakian, I.A. Pretty, R.P. Ellwood

Hooipin.Chew@postgrad.manchester.ac.uk

School of Dentistry, University of Manchester, UK

Enamel demineralisation due to dental erosion can be quantified non-invasively using OCT. Like other optical methods, OCT benefits from a reference area for comparison with the eroded areas and also to account for systematic errors. The aim of this study was to compare changes in surface characteristics of eroded surfaces with OCT using two in-vivo employable reference methods and surface microhardness. The two reference methods were using (1) moistened surfaces and (2) a dentin bonding agent coated surfaces. 12 human incisors were embedded in resin except for a $5 \times 5 \mathrm{~mm}$ window at the labial surface. Half of the window was coated with a dentin bonding agent, Xeno (3M Espe) while the other half was left exposed. Baseline measurements were taken with OCT and surface microhardness. The OCT system OCS1300SS (Thorlabs Ltd, UK) and a Knoop indenter were used. Specimens were immersed in orange juice $(\mathrm{pH} 3.8 \pm 0.1)$ in 10 minute-stepwise periods of up to $60 \mathrm{~min}$. After every $10 \mathrm{~min}$, the teeth were rinsed with water, dried and a measurement of the coated and exposed area was taken. The exposed area was then remoistened with water and another measurement was taken. Paired t-tests were used to analyse differences between baseline and each interval of erosion. Significant differences $(p<0.05)$ from baseline were found from 10-minute erosion onwards for all three methods except for the 60-minutes erosion interval for the moistened surface method. At 10-minute erosion, surface microhardness change was $0.23 \pm 0.05 \mu \mathrm{m}$ (mean $\pm \mathrm{SE})(\mathrm{p}=0.001)$ and the intensity ratio change of OCT backscattered light was 0.16 $\pm 0.04(\mathrm{p}=0.008)$ and $0.17 \pm 0.07(\mathrm{p}=0.04)$ for the coated and moistened methods, respectively. It is concluded that both reference methods can be used to reference in-vivo OCT erosion measurements.

Funding: HP Chew acknowledges funding from HLCB scheme of University of Malaya and the Ministry of Higher Education of Malaysia. 


\section{6 \\ Impact of Acidic Beverages on Prevalence of Dental Erosion in Young Adults Living in Romania}

A. Bor * B. Kerekes-Máthé, Z. Fazakas, M. Székely

andreeamolnar@yahoo.com

University of Medicine and Pharmacy, Târgu-Mure, Romania

In recent years there has been a very large increase in the consumption of soft drinks in Romania. The aim of this study was to assess the consumption of acidic beverages and the prevalence of dental erosion in young adults living in Târgu-Mure, Romania. The cross-sectional study was carried out involving 243 healthy adults. The subjects, aged 18 - to 27 -years, were randomly selected and information on dietary factors where gathered by a multiplechoice questionnaire. The tooth erosion index proposed by Lussi [Lussi: Eur J Oral Sci 1996;104:191-198] was used for the full mouth recording. Enamel loss was the most prevalent type of erosive tooth wear (grade 1). Data analysis included descriptive statistics on distribution and severity of dental erosion. $76 \%$ of subjects were consuming usually more than $400 \mathrm{ml}$ acidic soft drinks/ day. The prevalence of dental erosion was $25 \%$. Regular exposure to acidic beverages was $88.3 \%$ of subjects with dental erosion. The findings suggest a high consumption of soft drinks in young adults. The prevalence of dental erosion in screened subjects was significantly higher in individuals exposed to frequent intake of acidic soft drinks (Fisher's exact test, $\mathrm{p}=0.008$ ). It may be concluded that consumption of acidic beverages on a regular base might represent a risk factor in dental erosion.

\section{7 \\ The Effect of Gastro-Esophageal Reflux Disease on Dental Erosion Occurence and Salivary Gland Activity \\ J. Jurga* ${ }^{*}$ H. Shaw \\ justynajurga@o2.pl \\ Department of Biomaterials and Experimental Dentistry, Poznan University of Medical Sciences, Poland}

The purpose of this study was to investigate the effect of gastroesophageal reflux disease on dental erosion development and salivary gland activity in patients with GERD. A total of 68 adult subjects were examined. Two study groups were established: 31 patients with GERD diagnosed by 24-h pH-metry (group G) and 37 healthy control subjects (group C). Ethical approval and patients written consent were obtained. Erosive tooth wear was assessed according to the modified Smith and Knight tooth wear index, salivary basal flow rate (15 min collection) and stimulated flow rate ( 5 min collection by mastication of wax pellets ) were measured. Data were analyzed by Mann-Whitney test ( $\mathrm{p} \leq 0.05)$. Results: The prevalence of entire dental erosion in GERD patients was statistically higher than in the control group (16.42 vs. $8.79 \%$ ). The distribution of erosive lesions confined to enamel (score 1) were found in $13.38 \%$ of $\mathrm{G}$ group and $8.14 \%$ of $\mathrm{C}$ group, score 2 was observed in $4.4 \%$ of $\mathrm{G}$ group and $0.65 \%$ of $\mathrm{C}$ group and score
3 was only detected in GERD patients (0.18\%). Patients with GERD had statistically lower basal flow rate $( \pm \mathrm{SD}, \mathrm{ml} ; 0.51 \pm$ $0.47)$ compared to healthy subjects $(0.63 \pm 0.21)$. No statistically significant differences were found between groups in stimulated flow rate (group G: $1.90 \pm 0.77$, group C: $2.12 \pm 0.71$ ). Conclusions: Data obtained in this study may suggest that GERD has an influence on dental erosion development and salivary gland activity in terms of quantitative changes of saliva output.

Supported by the Polish Ministry of Science and Higher Education (No. N403 048 31/2421).

\section{8 \\ Prevalence of Occlusal Tooth Wear and Its Relationship to Abfraction: Observations in an Ancient Culture

\author{
I. Urzua ${ }^{\mathrm{a}, *}{ }^{*}$ R. Cabello ${ }^{\mathrm{a}}$, G. Rodriguez $^{\mathrm{a}}$, C. Meller $^{\mathrm{b}}$ \\ ivanurzua@yahoo.com \\ aDepartment of Restorative Dentistry, University of Chile, \\ Santiago, Chile; ${ }^{\mathrm{b}}$ Department of Conservative Dentistry, \\ Eberhard Karls University, Tübingen, Germany
}

The aim of the present pilot study was to evaluate the frequency of occlusal tooth wear and the presence of abfraction in an ancient culture (approx. 500 AD) that inhabited the Atacama Desert in Chile. The examination was performed on adult human skeletal remains of the pre-Columbian archaeological site of San Pedro de Atacama. A total of 99 human remains were eligible for inclusion by fulfilling the appropriate criteria. The latter were skeletons with complete head and gnathic remains, from which the prevalence of occlusal tooth wear and abfraction was recorded. The results showed that all analyzed subjects presented signs of occlusal wear, with $18.5 \%$ (95\% CI: $17.1 ; 20.1)$ of the teeth affected. A maximum of 32 and a minimum of 6 affected teeth per gnathic remain was observed. All remaining teeth, in $78.0 \%$ of the subjects, presented occlusal wear and in $19.2 \%$ of them, more than $80 \%$ of the teeth were affected. On the other hand, abfractive lesions were not found in any of the examined human remains. Overall, in spite of the fact that the prevalence of occlusal wear was high, none of the analyzed teeth presented signs of abfraction-like lesions. Further examinations are required to achieve more knowledge about the reasons, implications and etiology of these findings. 
Abrams, S.H. 147

Abuzar, M.A. 130

Acuña, A. 3

Adams, G.G. 17, 130

Afonso, R.L. 28

Ahme, F. 35

Akpata, E.S. 131

Al-Ahmad, A. 113

Alammari, M.R. 112

Aleksejûnienë, J. 57

Alex, V. 158

Alkilzy, M. 49

al Muhammad, M. 162

Alsane, M. 131

Altarakemah, Y. 131

Altenburger M.J. 113

Alton, J. 30

Alves, L.S. 118

Amaechi, B.T. 61, 87, 147

Amaral, R.C. 76

Amorim, R.G. 6, 148

Amsallem, E. 82

Anabtawi, M.Z. 61

Andaló, F.A. 169

Anderson, P. 35, 36, 39

Ando, M. 136

Arab, K. 143

Araujo, F.B. 37, 106

Arkader, R.J. 127

Armfield, J. 80

Arthur, R.A. 91

Arvin, E. 60

Attin, T. 65, 70, 125, 150, 176, 179

Auclair, C. 82

Azevedo, M.S. 44

Bachanek, T. 34

Bailey, D.L. 130

Bakhshandeh, A. 50

Bakht, K. 101

Bánóczy, J. 73

Barber, A.H. 35

Barbour, M. 36

Bardow, A. 60

Batterham, A. 79

Bavaresco, C.S. 107

Becker, K. 150

Belini, M.R. 78

Bento, L.W. 106

Berard, M. 102, 156

Berthold, L. 33

Bertolini, M. 63
Bigeard, L. 149, 166

Bittar, J. 6

Blair, Y.I. 18, 19

Boca, C. 141

Boldieri, T. 133, 137

Bonanato, K. 4

Bonifácio, C.C. 52

Bor, A. 186

Borromeo, G.L. 17

Borutta, A. 93

Boteva, E. 56

Bottenberg, P. 21, 141

Boudas, R. 149

Bouslimani, A. 159

Braga, J.A.P. 94

Braga, M.M. 52, 114, 123

Brighenti, F.L. 90

Brisset, L. 149, 166

Broukal, Z. 5

Brukienë, V. 57

Bruvo, M. 60

Buchalla, W. 150

Buchgraber, B. 115

Buchmann, N. 158

Buckley, C. 105

Bueno, M. 107

Burnett, G. 101

Butler, A. 24

Buzalaf, M.A.R. 22, 26, 67, 68, $78,157,170,178,180,181$

Cabello, R. 3, 20, 81, 188

Cagetti, M.G. 7

Callaway, A. 93

Campus, G. 7

Cankar, K. 155

Cantagallo, C.F. 28

Carlén, A. 11

Carvalho, C.B.M. 153

Carvalho, E.R.T. 63

Carvalho, J.C. 21

Carvalho, K.M. 153

Casagrande, L. 37, 106

Castro, L.P. 77

Catani, D.B. 169

Ccahuana-Vásquez, R.A. 91

Cenci, M.S. 44

Chalas, R. 34

Chandrappa, P. 105

Charone, S. 22

Chave, R. 16

Chawla, N. 146

Chazel, J.C. 8
Chew, H.P. 185

Choi, Y.H. 97

Chow, L.C. 75

Chung, S.Y. 97

Churchley, D. 30

Cismak, A. 33

Cloitre, T. 160

Cloivos, C. 22

Cochrane, N.J. 39, 130

Collart Dutilleul, P.Y. 16

Comar, L.P. 26, 67

Cooper, L. 30, 43

Cordeiro, R.C.L. 133, 137, 163

Cornelis, J. 141

Corona, S.A.M. 175

Cortes, A. 134

Côrtes, D.F. 127

Costa-Silva, C.M. 163

Crombie, F.A. 161

Cuisinier, F.J.G. 159, 160

Cury, J.A. 74, 76, 91, 103, 104, 169

Dalpian, D.M. 37, 106

Danellon, M. 28

Danelon, M. 77

Dashper, S.G. 92

Davis, G.R. 38, 39

de Azevedo, R.B. 108

Declerck, D. 21

Declerck, N. 141

Deery, C. 128

de Josselin de Jong, E. 100, $101,112,138,139,140$

Del Bel Cury, A.A. 74, 76, 104

Delbem, A.C.B. $28,77,90,171$

Delija, B. 116

de Soet, J.J. 63

Deville de Périère, D. 159

Diep, H.H. 152

Diniz, M.B. 133, 137

Do, L.G. 80

Doi, T. 9

Doméjean, S. 119

Dörfer, C.E. 41

Douglas, G. 124, 128

Dowker, S.E.P. 38

Dria, K.J. 153

Duggal, M.S. 83

Dukic, W. 116

Eberhardt, C. 121

Eckert, G.J. 120, 136
Eduardo, C.P. 179

Eggertsson, H. 128

Ekstrand, K.R. 48, 50, 60, 123, $124,128,131,134,135$

Elliott, J.C. 38

Ellwood, R.P. 127, 134, 135, 168, 185

Elman, G. 147

Espelid, I. 45

Esteves-Oliveira, M. 179

Evans, M. 29

Eversole, S.L. 71

Exterkate, R.A.M. 88

Faber, J. 148

Faller, R.V. 71

Fazakas, Z. 186

Ferreira Zandona, A.G. 120, 128

Fickenscher, V. 173

Figueiredo, M.J. 6

Figueiredo, M.S. 94

Filoche, S.K. 99

Finderle, Z. 155

Finer, Y. 147

Fioretti, F. 151

Fonteles, C.S.R. 153

Franzon, R. 37, 106

Freitas, A.Z. 142

Freitas, L.S.F. 90

Frencken, J.E. 6, 148

Frohn, J. 145

Fujita, K. 85

Gamboa, L.F. 128

Ganss, C. 172, 173, 183

Ganter, P. 113

Garcia, J. 147

García-Godoy, F. 106

Gerbaud, L. 82

Gergely, C. 160

Giacaman, R.A. 62

Gomes, A.S.L. 142

Gomes, M.F. 67

Gomez, J. 135

Gomez, S.S. 81

Gonçalves, R.M. 22

González-Cabezas, C. 143

Gorbatova, M.A. 2

Götz, G. 73

Grausi, V. 159

Grizzo, L.T. 22, 78

Groisman, S. 22, 63

\section{KARGER}

(c) 2010 S. Karger AG, Basel

Fax +41 613061234

E-Mail karger@karger.ch

www.karger.com 
Grunau, O. 172

Gruythuysen, R.J.M. 53

Guerin-Deremaux, L. 102, 156

Guglielmi, C.A.B. 59

Häberlein, I. 95, 98

Hannas, A.R. 157, 180, 181

Hannig, M. 162

Hara, A.T. $174,175,184$

Haughey, A.-M. 182

Hauke, M. 95, 98

Heinrich, J. 164

Heinrich-Weltzien, R. 51, 84, 164

Heinzel-Gutenbrunner, M. 144

Hellen, W.M.P. 147

Hellwig, E. 113

Henin, L. 141

Hesse, D. 52

Hickel, R. 51, 164

Hiestand, B. 65

Higham, S.M. 30, 43, 100, 101, 112

Hirtz, C. 159

Holme, B. 66

Holmgren, C.J. 84

Hoogstraten, J. 54, 64

Hopcraft, M.S. 13, 14, 130

Hope, C.K. 100, 101

Hopfenmuller, W. 46

Hornby, K. 29

Hoßdorf, W. 177

Hove, L.H. 66, 126

Hug, I. 132

Huysmans, M.C.D.N.J.M. 72

Ibarra, D. 62

Igreja, B.B. 28

Iijima, S. 32

Iijima, Y. 32

Imparato, J.C.P. 52, 59, 114, 123

Inaba, D. 138, 139, 140

Inagaki, S. 85

Inukai, J. 27

Ismail, A. 131

Italiani, F.M. 78, 178

Ivkovski, L. 109

Jablonski-Momeni, A. 144

Jan, J. 155

Jaruszewski, L. 145

Jehl, M. 166

Jeremias, F. 163

Jin, K. 9

Joiner, A. 29

Jones, A. 40

Jonski, G. 69

Jurga, J. 187

Juric, H. 122
Kadic, S. 116

Kakaboura, A. 129

Kambara, M. 9

Kaminski, M. 12

Karaiasheva, D. 56

Karlinsey, R.L. 23

Karthikeyan, R. 87

Kato, K. 27, 89, 96

Kato, M.T. 157, 178, 180, 181

Kawabata, S. 85

Kawachi, C.D.P. 104

Kawamura, Y. 89

Kawasaki, K. 9

Kelly, S.A. 105

Keltsch, J. 41

Kerekes-Máthé, B. 186

Kielbassa, A.M. 25, 31

Kiesow, A. 33

Kim, E.Y. 47

Kim, J.H. 97

Kim, S. 47

Klijn, C. 72

Klimek, J. 172, 173, 183

Kneist, S. 93

Kobayashi, C.A.N. 78

Koga-Ito, C.Y. 90, 94

Kolker, J. 128

Komiyama, E.Y. 94

Korwanich, N. 168

Kosoric, J. 35

Krause, J. 150

Krejsa, O. 5

Krushinsky, C. 121

Kuczumow, A. 34

Kühnisch, J. 51, 164

Küpper, H. 93

Kvam, K. 126

Lamarre, J.R. 42

Lampert, F. 179

Lara, J.S. 128

Larroque, C. 159

Leal, S.C. $6,108,148$

Lee, A. 17

Lee, S.Y. 47

Lee, Y.E. 97

Lefranc-Millot, C. 102, 156

Lehmann, A. 162

Leite, A.L. 157, 178, 180

Lelong, N. 12

Lenčová, E. 5

Lennon, Á.M. 150

Levallois, B. 55, 160

Levy, F.M. 67, 68

Li, S. 102,156

Lingawi, H. 36

Lingström, P. 7, 11

Lintner, A. 115

Lippert, F. 24, 30, 33, 174

Liu, S.-W. 92
Longbottom, C. 128, 135, 182

Longhi, S. 55

Longo, J.P.F. 108

Lulic, O. 116

Lund, R.G. 44

Lussi, A. 132, 133, 137, 145

Luz, M.A.A.C. 59

Lynch, R.J.M. 24, 30, 33, 43

Mach, D. 164

Macioce, V. 102, 156

Mackey, A.C. 23

Macpherson, L.M.D. 18, 19

Madléna, M. 73

Magalhães, A.C. 26, 65, 67, 68, 170,181

Maguire, A. 79

Maia, A.M.A. 142

Makhija, S.K. 61

Malinowski, M. 83

Maltz, M. 107, 118

Manareli, M.M. 171

Mandelis, A. 147

Mannaa, A. 11

Manton, D.J. 146, 161

Marin, L.M. 58

Mariño, R. 81

Marinova, M. 56

Martignon, S. 48, 50, 58, 128, 134, 135

Martin, G.C. 101

Martin, M. 160

Martinez-Mier, E.A. 105

Martinhon, C.C.R. 171

Márton, S. 73

Matos, B.M. 94

Matos, R. 114, 123

Matosic, Z. 122

Matsumoto, M. 86

Matsumoto-Nakano, M. 85

Maupome-Carvantes, G. 121

McCall, D.R. 18, 19

McCullough, M.J. 17

McGrady, M.G. 168

McMahon, A.D. 18,19

Meller, C. 188

Mendes, F.M. 114, 123

Messer, L.B. 146

Meyer-Lueckel, H. 25, 41, 46, 158

Michailesco, P. 8

Milford, E. 13, 14

Mitropoulos, P. 129

Miyake, T. 9

Moi, G.P. 103

Monse, B. 84

Morgan, M.V. 13, 14

Moron, B.M. 26

Mortazavi, S. 167

Moura-Leite, F.R. 4
Mujica, V. 62

Mukai, M. 27

Mulder, J. 6

Muller, D. 166

Müller, M.J. 10

Musset, A.M. 12, 149, 151, 166

Nabet, C. 12

Nagayama, K. 85

Nagy, G. 73

Nakagaki, H. 27, 89, 96

Nakano, K. 86

Narayan, M.J. 14

Narayan, M.L. 13

Nemeth, L. 110

Neuhaus, K.W. 145

Neutard, L. 172, 183

Nguyen, A. 40

Noin, S. 167

Nomura, R. 86

Novacic, A. 122

Novaes, T.F. 114, 123

Nowak, J. 34

Nunn, J. 1

Nyssen, M. 21

Nyvad, B. 145

O'Hare, W.T. 79

Okoye, L.O. 15

Oliveira, E. 107

Omid, N. 79

Ooshima, T. 85, 86

Ou, Y. 96

Owens, G.J. 43

Ozawa, T. 32

Padilla, A. 58

Paes Leme, A.F. 103

Paiva, S.M. 4

Panayotov, I. 160

Paris, S. 41, 46, 158

Pastbin, M.U. 2

Pelissier, B. 55

Pereira, A.F.F. 170

Pereira, H.A.B.S. 178

Pereira, J.C. 181

Perfect, E. 182

Pessan, J.P. 78

Petzold, M. 33

Picek, V. 116

Pieper, K. 144

Pinheiro, S.L. 59

Pitts, N.B. 128, 131, 182

Plachta-Danielzik S. 10

Poorterman, J.H.G. 54

Popovska, L. 109

Pordeus, I.A. 4

Preisser, J.S. 61

Pretty, I.A. 168, 185

Proshkina, E.I. 2 
Qvist, V. 58

Raggio, D.P. 52, 59, 114

Rahiotis, C. 129

Ramos-Jorge, M.L. 4

Raskin, A. 55

Rencker, M. 151

Reynolds, E.C. 39, 92, 161

Ribeiro, T.R. 153

Ribeiro, Z.E.A. 94

Ricketts, D.N.J. 124, 128, 131

Rios, D. 67, 68

Ritter, A.V. 61

Rizzante, F.A.P. 68

Roberts-Thomson, K.F. 80

Robinson, C. 89

Rocha, C.T. 175

Rochel, I.D. 170

Rodrigues, J.A. 132, 133, 137

Rodríguez, G. 3, 20, 188

Rölla, G. 69

Romieu, G. 160

Roos, M. 65, 70

Rosen, S.M. 144

Ruben, J.L. 72

Ruiz, J.A. 58

Rupf, S. 162

Rusin, R.P. 98

Sacco, G. 7

Sagheri, D. 1

Sakagami, T. 136

Sakakibara, S. 96

Sale, S. 7

Salvador, M.J. 90

Sampaio, J.E.C. 31

Santiago, E. 120

Santos-Pinto, L. 137, 163

Sassaki, K.T. 77

Schäfer, B. 162

Schenkel, A.B. 111

Schiffner, U. 177

Schindler, A. 162

Schipper, H.M. 144

Schlueter, N. 172, 173, 183

Schmid, B. 95, 98
Schmidt, F. 93

Schulte, A.G. 82, 141

Schumacher, G.E. 75

Séllos, M.C. 117

Sener, B. 65

Shaw, H. 187

Shin, J.H. 47

Shugars, D.A. 61

Sibilia, J. 149

Silva, N. 3

Silva, T.C. 170

Silva, W.J. 104

Siminoni, A.R. 108

Simionato, M.R.L. 59

Sissons, C.H. 99

Sivagurunathan, K. 147

Sixou, M. 12

Slade, G.D. 152

Smith, P.W. 112

Sohn, W. 128

Song, K.B. 97

Sotirovska-Ivkovska, A. 109

Soto-Rojas, A.E. 121

Souza, J.F. 133, 163

Souza, J.G. 170

Souza-e-Silva, C.M. 178

Soviero, V.L.M. 117, 127, 165

Spencer, A.J. 80, 152

Splieth, C.H. 10, 49

Spliid, H. 60

Srisilapanan, P. 168

Stacey, M.A. 130

Stachniss, V. 141, 144

Städtler, P. 115

Stafford, S.M. 83

Stenhagen, K.R. 66, 126

Stillhard, A. 176

Stöger, H. 95, 98

Stookey, G.K. 23

Strohmenger, L. 7

Suyamaa, E. 32

Suzuki, A. 32

Swain, M.V. 40

Szádeczky, B. 73

Székely, M. 186
Tabchoury, C.P.M. 74, 76, 91

Takeshita, E.M. 77

Tamura, K. 96

Tamura, T. 32

Taniguchi, N. 86

Tantbirojn, D. 98

Tapia, P. 62

Tassery, H. 55

Tauböck, T.T. 176

Techert, S. 150

Tedesco, A.C. 108

Teixeira, T.S. 52

Tellez, M. 58

ten Cate, J.M. 74, 88

Tenuta, L.M.A. 74, 75, 76, 104, Wall-Manning, G.M. 99 169

Terrer, E. 55

Thabuis, C. 102,156

Thiering, E. 164

Thompson, V.P. 111

Thuy, T.T. 89

Tjäderhane, L. 157, 181

Tomiyama, K. 88

Toumba, K.J. 83

Tramini, P. 8, 16, 82

Trindade, C.P. 165

Truyen, B. 141

Tschoppe, P. 25, 31

Tubert-Jeannin, S. 82, 119

Turssi, C.P. 184

Tveit, A.B. $45,66,126$

Uene, M. 9

Unk, D. 72

Uribe, S. 81

Urzúa, I. 3, 20, 188

Valappil, S.P. 43

Vale, G.C. 74

Vale, M.P. 4

Valvarcel, J. 16

van Bekkum, M. 99

van der Veen, M.H. 138, 139, 140

van de Sande, F.H. 44

van Loveren, C. 53, 64
Van Nieuwenhuysen, J.P. 21

Vanobbergen, J. 21

van Palenstein Helderman, W.H. 53,84

Vergnes, J.N. 12

Vermaire, J.H. 54, 64

Véronneau, J. 16, 42, 154

Vidnes-Kopperud, S. 45

Vintan, P. 8

Vogel, G.L. 75

von Hinckeldey, J. 173, 183

Vougiouklakis, G. 129

Wada, K. 86

Wang, J. 124

Warita, S. 140

Wassif, H.S. 38

Weber, C.M. 118

Wegehaupt, F.J. 70, 125, 176

Wiegand, A. 26, 65, 70, 179

Willershausen, B. 93

Wils, D. 102, 156

Witzke, H. 125

Wong, L. 99

Wrbas, K.-T. 113

Yoder, K.M. 121

Yoo, S.G. 47

Young, A. 69

Yu, H. 26, 70, 179

Zabokova, E. 109

Zakian, C.M. 185

Zalizniak, I. 161

Zandim, D.L. 31

Zarella, B.L. 157

Zaruba, M. 70

Zero, D.T. 105, 120, 128, 136, 174,175

Zhong, B. 102, 156

Zillmann, G. 20

Zohoori, V. 79

Zou, W. 40

Zuanon, A.C.C. 163 